\title{
Echocardiographic predictors for recurrence of ischemic mitral regurgitation after restrictive annuloplasty
}

Citation for published version (APA):

van Garsse, L. (2013). Echocardiographic predictors for recurrence of ischemic mitral regurgitation after restrictive annuloplasty. [Doctoral Thesis, Maastricht University]. Universitaire Pers Maastricht. https://doi.org/10.26481/dis.20130627lg

Document status and date:

Published: 01/01/2013

DOI:

10.26481/dis.20130627lg

Document Version:

Publisher's PDF, also known as Version of record

\section{Please check the document version of this publication:}

- A submitted manuscript is the version of the article upon submission and before peer-review. There can be important differences between the submitted version and the official published version of record.

People interested in the research are advised to contact the author for the final version of the publication, or visit the DOI to the publisher's website.

- The final author version and the galley proof are versions of the publication after peer review.

- The final published version features the final layout of the paper including the volume, issue and page numbers.

Link to publication

\footnotetext{
General rights rights.

- You may freely distribute the URL identifying the publication in the public portal. please follow below link for the End User Agreement:

www.umlib.nl/taverne-license

Take down policy

If you believe that this document breaches copyright please contact us at:

repository@maastrichtuniversity.nl

providing details and we will investigate your claim.
}

Copyright and moral rights for the publications made accessible in the public portal are retained by the authors and/or other copyright owners and it is a condition of accessing publications that users recognise and abide by the legal requirements associated with these

- Users may download and print one copy of any publication from the public portal for the purpose of private study or research.

- You may not further distribute the material or use it for any profit-making activity or commercial gain

If the publication is distributed under the terms of Article $25 \mathrm{fa}$ of the Dutch Copyright Act, indicated by the "Taverne" license above, 
Echocardiographic predictors for recurrence of ischemic mitral regurgitation after restrictive annuloplasty 
Financial support by Stichting Hartsvrienden RESCAR for the publication of this thesis is gratefully acknowledged.

Additional financial support was kindly provided by Edwards Lifesciences, Cardiac Care, JenaValve Technology, Maquet, Sorin Group and Johnson \& Johnson Medical Companies

(C) Copyright Leen Van Garsse, Maastricht 2013.

All rights reserved. No part of this thesis may be reproduced of transmitted in any form of by any means, electronic or mechanical, including photocopying, recording, or any information storage or retrieval system without permission from the author, or when appropriate, from the publishers of the publications.

Alle rechten voorbehouden.

Niets uit deze uitgave mag worden verveelvoudigd, opgeslagen in een geautomatiseerd gegevensbestand en/of openbaar gemaakt in enige vorm of op enige wijze, hetzij elektronisch, mechanisch, door fotokopieën, opnamen of op enige andere manier zonder voorafgaande schriftelijke toestemming van de uitgever.

Omslagillustratie "Child, I'm your mother..." (gedicht pag 5) door Birte Van Garsse, beeldend kunstenaar Omslagontwerp: Datawyse

Vormgeving en druk: Datawyse | Universitaire Pers Maastricht

ISBN 9789461592330 


\title{
Echocardiographic predictors for recurrence of ischemic mitral regurgitation after restrictive annuloplasty
}

\author{
PROEFSCHRIFT \\ ter verkrijging van de graad van doctor aan de Universiteit Maastricht, \\ op gezag van de Rector Magnificus, Prof. Dr. L.L.G. Soete, \\ volgens het besluit van het College van Decanen, \\ in het openbaar te verdedigen, \\ op donderdag 27 juni 2013, om 16.00 uur \\ door \\ Leen Van Garsse
}

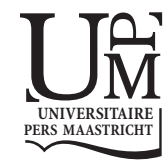




\section{Promotor}

Prof. Dr. J.G. Maessen

\section{Copromotor}

Dr. S. Gelsomino

\section{Beoordelingscommissie}

Prof. Dr. F.W. Prinzen (voorzitter)

Prof. Dr. H.J.G.M. Crijns

Prof. Dr. M.W. de Haan

Prof. Dr. R.J.M. Klautz 
Child,

I'm your mother, And I have to introduce

You to the world And to his fight Like in a storm I know that it's noise You will be hurled Away by its might I'll have to attempt To protect that innocence Let the world not tempt Your golden innocence

(Source unknown)

Aan Tomas, Carmen-Anne en Pieter-William 



\section{Contents}

$\begin{array}{lc}\text { Abbreviations List } & 8\end{array}$

$\begin{array}{lll}\text { Chapter } 1 & \text { Introduction } & 11\end{array}$

Chapter 2 Importance of anterior leaflet tethering in predicting the 53 recurrence of ischemic mitral regurgitation after restrictive annuloplasty.

Chapter 3 Impact of preoperative anterior leaflet tethering on the 65 recurrence of ischemic mitral regurgitation and the lack of left ventricular reverse remodeling after restrictive annuloplasty.

Chapter 4 Left ventricular dyssynchrony is associated with recurrence of ischemic mitral regurgitation after restrictive annuloplasty.

Chapter 5 Systolic papillary muscle dyssynchrony predicts recurrence of mitral regurgitation in patients with ischemic cardiomyopathy undergoing mitral valve repair.

Chapter 6 Tethering symmetry reflects advanced left ventricular mechanical dyssynchrony in patients with ischemic mitral regurgitation undergoing restrictive mitral valve repair.

Chapter 7 Left ventricular strain in chronic ischemic mitral regurgitation in relation to mitral tethering pattern.

Chapter 8 Left atrial strain and strain rate before and following restrictive annuloplasty for ischemic mitral regurgitation evaluated by twodimensional speckle tracking echocardiography.

Chapter 9 Conclusions

Chapter 10 Summary

Samenvatting (Summary in Dutch)

Dankwoord

Curriculum vitae

List of publications 


\section{Abbreviations List}

$\alpha$

$\alpha_{\mathrm{EX}}$

$\beta$

$\beta_{\mathrm{EX}}$

$Y$

$\varepsilon$

AML

ALPM

ALPM-LS

ALPM-WMSI

AUG

BMI

BSA

CABG

CCS

CD

$\mathrm{CH}$

$\mathrm{Cl}$

CIMR

$\mathrm{CL}$

DT

DYS-PAP

EDD

EDV

EDVI

EROA

ESD

ESV

ESVI

FMR

GLS

$\mathrm{GLS}_{\text {peak }}$

IMR

IVCT

IVRT
Anterior mitral leaflet tethering angle

Anterior mitral leaflet excursion angle

Posterior mitral leaflet tethering angle

Posterior mitral leaflet excursion angle

Bending angle

Left atrial peak systolic strain

Anterior mitral leaflet

Anterolateral papillary muscle

Anterolateral papillary muscle longitudinal strain

Anterolateral papillary muscle wall motion score index

Area under the curve

Body mass index

Body surface area

Coronary artery bypass grafting

Canadian Cardiovascular Society

Coaptation distance

Coaptation height

Confidence interval

Chronic ischemic mitral regurgitation

Coaptation length

Deceleration time at early diastolic filling

Papillary muscle systolic dyssynchrony

End-diastolic diameter

End-diastolic volume

End-diastolic volume index

Effective regurgitant orifice area

End-systolic diameter

End-systolic volume

End-systolic volume index

Functional mitral regurgitation

Global longitudinal strain of left ventricle

Peak global longitudinal strain

Ischemic mitral regurgitation

Isovolumetric contraction time of left ventricle

Isovolumetric relaxation time of left ventricle 


\begin{tabular}{|c|c|}
\hline IQR & Interquartile range \\
\hline LA & Left atrium \\
\hline LAD & Left atrial diameter \\
\hline LAEF & Left atrial ejection fraction \\
\hline LAV $_{\text {CONTR }}$ & Contraction left atrial volume \\
\hline LAV $_{\text {MAX }}$ & Maximum left atrial volume \\
\hline $\mathrm{LAV}_{\mathrm{MIN}}$ & Minimum left atrial volume \\
\hline $\mathrm{LAV}_{\mathrm{p}}$ & Left atrial volume prior to atrial contraction \\
\hline LAV $_{\text {P-EMPT }}$ & Passive-emptying left atrial volume \\
\hline LAV $_{\text {RES }}$ & Reservoir left atrial volume \\
\hline LV & Left ventricle \\
\hline LVEF & Left ventricular ejection fraction \\
\hline LVGRR & Left ventricular geometrical reverse remodeling \\
\hline LVR & Left ventricular remodeling \\
\hline LVRR & Left ventricular reverse remodeling \\
\hline MPI & Myocardial performance index \\
\hline MR & Mitral regurgitation \\
\hline MV & Mitral valve \\
\hline NYHA & New York Heart Association \\
\hline PISA & Proximal isovelocity surface area \\
\hline PM & Papillary muscle \\
\hline PML & Posterior mitral leaflet \\
\hline PMPM & Posteromedial papillary muscle \\
\hline PMPM-LS & Posteromedial papillary muscle longitudinal strain \\
\hline PMPM-WMSI & Posteromedial papillary muscle wall motion score index \\
\hline RA & Restrictive annuloplasty \\
\hline ROC & Receiver operating characteristic curve \\
\hline RF & Regurgitant fraction \\
\hline RV & Regurgitant volume \\
\hline$S I_{D}$ & Sphericity index (diastolic) \\
\hline $\mathrm{SI}_{\mathrm{S}}$ & Sphericity index (systolic) \\
\hline SR & Strain rate \\
\hline $\mathrm{SR}_{\mathrm{A}}$ & Peak late diastolic strain rate \\
\hline $\mathrm{SR}_{\mathrm{E}}$ & Peak early diastolic strain rate \\
\hline $\mathrm{SR}_{\mathrm{p}}$ & Peak systolic strain rate \\
\hline 2D-STE & Two-dimensional speckle tracking echocardiography \\
\hline TA & Tenting area \\
\hline
\end{tabular}


Transesophageal echocardiography

TTE

Transthoracic echocardiography

UMRA

Undersized mitral ring annuloplasty

WMSI

Wall motion score index 
Chapter 1

Introduction

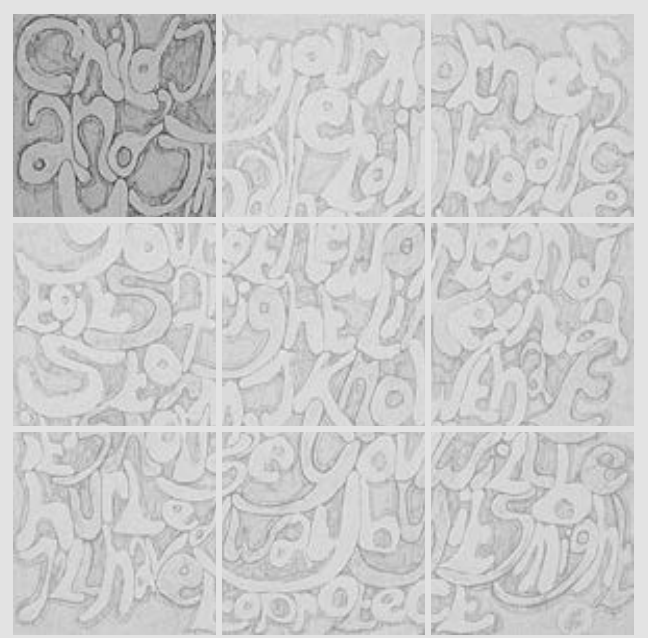


Chronic ischemic mitral regurgitation (CIMR) is a functional insufficiency of the mitral valve (MV) related to changes in the geometry and performance of the left ventricular wall as a result from ischemic heart disease ${ }^{1,2}$. The last few decades CIMR has been treated by restrictive annuloplasty (RA), combined with coronary bypass grafting $(C A B G)^{3}$. However, despite the enthusiasm for low perioperative mortality rate, high initial surgical success rate (no residual mitral regurgitation - MR) and the positive effects on survival and functional symptomatic status ${ }^{4-7}$, an increasing number of studies have clearly demonstrated that $R A$ is subject to variable rates of recurrence of $M R^{4}$, ${ }^{8-10}$. Mechanisms for the recurrence were described by several authors and progressive left ventricular adverse remodeling (LVR), local or global, seems to be the crowbar to distort the balance of valve closure, causing recurrence of $M R^{11,12}$. However, recent studies have confirmed that some patients with CIMR may benefit from RA ${ }^{6,7}$. To select which patient will benefit from RA the search for preoperative predictors of recurrent MR goes on.

\section{Definition of chronic ischemic mitral regurgitation (CIMR)}

The mitral valve complex can be described by five anatomical structures that are involved in the normal function of the mitral valve: 1) the mitral annulus, 2) mitral leaflets, 3) the chordae tendineae, 4) the papillary muscles and 5) the left ventricle. In mitral valve disease, each of these anatomical structures can be involved separately or contemporarily, causing MR, mitral stenosis (MS) or a combination of MR and MS. For the definition of CIMR it is important to stress the presence of the three indispensable components of MR: functional, ischemic and chronic.

1. The mechanism of $M R$ is functional, in contrast to organic $M R(O M R)$ : Functional mitral regurgitation (FMR) means that the mitral leaflets and chordae tendineae are primarily not involved in the cause of the disease. The closure of the MV is insufficient because of dysfunction of the annulus, the papillary muscles and the left ventricle. FMR can be defined as type I or type IIIb leaflet dysfunction following Carpentier's classification ${ }^{13}$. (FIGURE 1)

However, in a later discussion, second-order chordae cutting will be described as one of the surgical treatment options for functional $\mathrm{MR}^{14}$, but this should not give the suggestion that the FMR process originates from dysfunction of the chordae. In contrast, if the mitral leaflets and chordae are affected by infective, rheumatic, degenerative (Barlow's disease and fibroelastic deficiency - FED) or rarely traumatic disease, and do cause the insufficiency of the MV closure, this is defined as OMR. In patients with MR arising from rheumatic disease, the mitral annulus, mitral leaflets, chordae and tip of papillary muscle are affected, leading to mitral leaflet retraction - type IIla leaflet dysfunction - following Carpentier's classification $^{15}$. In Barlow's disease, mitral leaflets and chordae present lesions of myxoid 
degeneration, which often affects the entire valve, including leaflet thickening, large redundant leaflets, chordal elongation or rupture and annular dilatation ${ }^{16}$. In contrast to Barlow's disease, patients with MR due to FED have a lack of connective tissue as the pathological mechanism that triggers leaflet and chordal thinning and eventual chordal rupture ${ }^{17}$, usually leading to prolapse of a single leaflet segment ${ }^{18}$. Finally, OMR can result from traumatic mitral disease, mostly affecting one of the PMs and requiring emergency intervention ${ }^{19}$, or infective mitral disease, characterized by structural injury to one or more compounds of the MV complex ${ }^{20}$
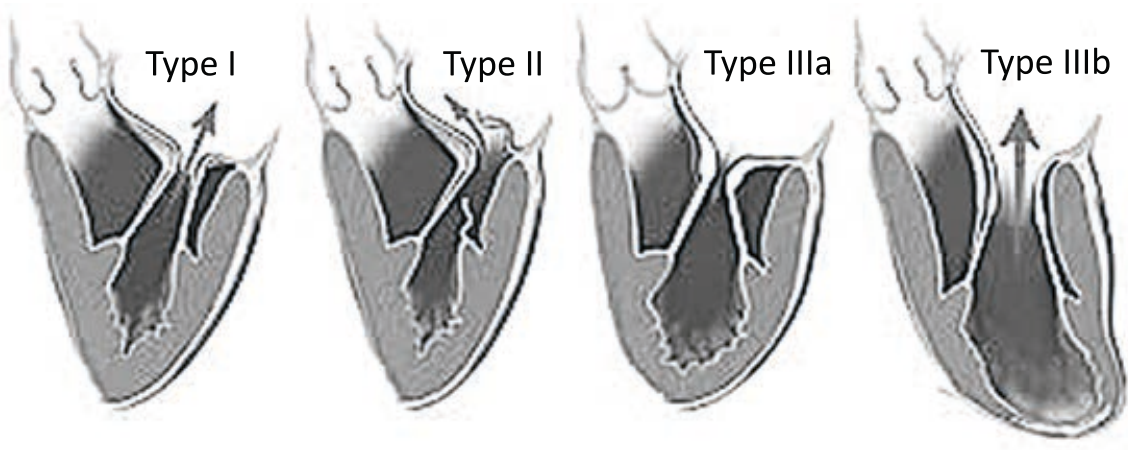

Figure 1. Mitral valve regurgitation: Classification of Carpentier ${ }^{13}$

2. The etiology of $M R$ is ischemic heart disease, in contrast to non-ischemic (idiopathic) heart disease. Ischemic mitral regurgitation (IMR) is a condition where mitral insufficiency is caused by coronary artery disease. Sequels of ischemia affect the left ventricular muscles, leading to failure of proper MV closure (this subject will be elucidated further in this thesis). In contrast, in patients with non-ischemic cardiomyopathies, also referred to as idiopathic cardiomyopathy, MR originates from a multifactorial etiology and can be provoked by genetic factors ${ }^{21}$, viral disease $^{22}$, medication (chemotherapy ${ }^{23}$ ), alcohol abuse ${ }^{24}$, drugs (e.g. cocaine ${ }^{25}$ ) and even in the postpartum period. Finally, it is important to distinguish IMR from MR which was originally caused by an organic etiology (OMR) but that can fortuitously be associated with coronary artery disease.

3. The presentation of $M R$ is chronic, in contrast to acute $M R$. Chronic mitral regurgitation (CMR) excludes regurgitation which is mostly the consequence of acute papillary muscle rupture caused by acute myocardial infarction (MI) ${ }^{26,27}$ or traumatic mitral injury ${ }^{19}$. On very rare occasions, acute MR is described with intact papillary muscles and is based only on systolic leaflet restriction. These cases are reported after inferior acute myocardial infarction, leading to cardiogenic shock. ${ }^{28}$ 
Acute ischemic mitral insufficiency is a life threatening disease, requiring emergency intervention ${ }^{26-28}$.

\section{Prevalence and incidence CIMR}

The prevalence of CIMR is difficult to calculate as a result of the heterogeneity of study data: the differences related to interval between $\mathrm{MI}$ and the occurrence of $\mathrm{MR}$, the reported techniques to diagnose (ventriculography versus echocardiography) and quantify MR (semi-quantitative versus quantitative assessment) and the study characteristics (observational, cohorts, etc.). This is illustrated by the varying incidence that has been reported on IMR during cardiac catheterization. Hickey et al. reported a $19 \%$ incidence of post-infarct IMR (3\% moderate to severe) ${ }^{29}$ among 11.748 patients with significant coronary artery disease, an incidence that was comparable with the findings of Lamas et al $\left(19.4 \%\right.$ overall and $4.5 \%$ moderate or severe MR) ${ }^{30}$. The incidence in the study by Tcheng et al. was higher, showing a $50 \%$ presence of moderately severe to severe $\mathrm{MR}^{31}$. In the latter study, which comprised 1.480 subjects and was performed within a 6-hour interval after the MI, MR was associated with a mortality of $24 \%$ at 30 days, $42 \%$ at 6 months and $52 \%$ at 1 year $(\mathrm{Cl}, 38 \%$ to $66 \%)$. Other studies were based on semi-quantitative echocardiographic measurements and reported a $6-12 \%$ incidence of moderate or severe MR in patients with ischemic heart disease ${ }^{32,33}$.

When assuming the presence of moderate or severe IMR to be $8 \%$ of the ischemic patients, an estimation of IMR prevalence can be made since American statistical data show a $7 \%$ (16,3 million patients) prevalence of coronary heart disease among the American adults ( $\geq 20$ years old $)^{34}$. By calculating, we found a presence of moderate or severe IMR in 1.3 million adults ( $\geq 20$ years old) in the United States. IMR has a prevalence of $1.1 \%$ in the adult American population.

\section{Outcome of patients with CIMR, without valve treatment}

As demonstrated by Felker et $\mathrm{al}^{35}$, ischemic cardiomyopathy should be considered as an isolated entity that is associated with a poorer outcome than a non-ischemic one ${ }^{36}$, 37. Indeed, it has been considered the main cause of heart failure, being the underlying cause in nearly $70 \%$ of patients with heart failure ${ }^{38}$.

Patel et al retrospectively studied 558 patients with advanced heart failure (NYHA class III or IV, EF < 35\%), with ischemic etiology in $71 \%$ of the patients (the remainder had idiopathic cardiomyopathy) ${ }^{39}$. Moderate or severe MR was found in $39 \%$ of the patients. The authors concluded that NYHA class was correlated with the severity of $M R$ and despite a significantly higher mortality in MR patients compared to those 
without, MR was not an independent risk factor of death. Moreover, the ischemic etiology was defined as an independent mortality risk factor.

When concentrating on the ischemic patient population, the natural history of patients with CIMR results in a poorer prognosis compared to patients with ischemic heart disease but without $\mathrm{MR}^{1,30}$. Recent studies of patients with CIMR showed a 5year survival of $60 \%-62 \%$ when mild or moderate MR and $23 \%-40 \%$ when severe

MR was present ${ }^{33,40}$. Tcheng et al. reported the outcome of patients that were diagnosed with IMR, early after the $\mathrm{MI}$ ( $<6$ hours interval). The patients in the study had a 1-year survival of $48 \%, 78 \%$ and $89 \%$ depending of their presentation with severe, mild or moderate and trivial MR, respectively ${ }^{31}$. These authors concluded that despite acute reperfusion by emergency balloon angioplasty, the high mortality in severe IMR patients could not be reduced to levels experienced by patients with lesser degrees of $\mathrm{MR}$, and valvular competence could not be restored. This suggests that LV remodeling is not being reconverted by only treatment of coronary ischemia. Di Mauro et al. confirmed the findings of Tcheng and colleagues in a surgical population by studying the outcome of isolated CABG in 140 propensity-matched ischemic patients (mean EF 27\%) with and without moderate or severe $\mathrm{MR}^{41}$. They concluded that moderate IMR has an important negative impact on either survival or quality of life of patients with severely impaired LV function whenever they are treated by CABG alone. Additionally, they propounded that their data could not demonstrate a better outcome after concomitant MV treatment. Although, studying a patient population with different characteristics than the group of Di Mauro, Kang et al. addressed this consideration comparing IMR patients undergoing PCI (NYHA mean 2,27 \pm 1.01 ; EF mean $40 \pm 12 \%$ ) or CABG (in $57 \%$ with mitral annuloplasty: NYHA mean $2.60 \pm 0.82$; EF mean $35 \pm 11 \%$ ). These authors concluded that surgical revascularization with concomitant mitral annuloplasty was associated with improved long-term event-free survival compared to CABG alone or $\mathrm{PCl}^{42}$.

\section{Pathophysiology of CIMR}

\section{The normal mitral valve function}

To understand normal MV function during the cardiac cycle, the physiological phases of diastole and systole will be briefly described in relation to the MV opening and closure. Mitral valve closure can be described as the coaptation of the anterior mitral leaflet $(A M L)$ and the posterior mitral leaflet $(P M L)$, a counter-pressure of the free edges of both leaflets. It is important to stress that the leaflet opening is primarily dictated by the pressure gradient between the left atrium and ventricle, while the normal mitral leaflet closure is a result of a three-dimensional leaflet interaction, based on a delicate balance between two forces: the leaflet closing forces (to induce 
leaflet coaptation) and the leaflet tethering forces (to prevent leaflet prolapse). The three-dimensional interaction of leaflets is a simplification of the reality since synchronization of papillary muscle contraction (the timing) acts as the fourth-dimension for MV closure ${ }^{43}$.

The cardiac cycle

Diastole can be divided in four phases: 1) the isovolumetric relaxation phase, during which the ventricular pressure rapidly declines without a significant change in volume, is the interval between the aortic valve closure and the opening of the $\mathrm{MV} ; 2$ ) the rapid-filling phase of the LV immediately after opening of the MV, 3) the slow-filling phase when passive filling pressure of the LV increases, and 4) the atrial contraction phase until the MV closure. Systole can be divided in two phases: 1) the isovolumetric contraction of the LV, during which the ventricular pressure rapidly increases without significant changes in volume; and, 2) the ejection phase, which starts with the opening of the aortic valve and stops when the aortic valve closes, when the pressure in the aorta exceeds the pressure in the LV. The MV opens when the left atrial pressure exceeds the left ventricular pressure after isovolumetric ventricular relaxation. The MV closes when the pressure in the filled LV exceeds the pressure in the LA.

The flow over the MV

Flow over the $M V$ can be described in two phases: 1) early diastolic flow is initially recognized as high blood flow during the last phase of ventricular relaxation, but later slows down when the pressure in the LV increases by passive elements affecting progressive filling of the LV and stiffness of the LV myocardium; and, 2) the late diastolic flow which is caused by the left atrial contraction and decreases by progressive filling pressure of the LV and stops with the closure of the MV.

\section{The left atrial function}

For the functional evaluation of the MV, the contribution of the left atrial wall can be described as the sixth compound of the MV complex: this is in terms of contraction, relaxation and dilatation ${ }^{44}$. To understand the left atrial function (which is subject of Chapter 8 in patients with IMR), the flow over the MV and the phases of global LA function are reported.

For left atrial global function, three phases can be described: 1) the reservoir phase, during which filling proceeds from the pulmonary veins, contemporaneous with ventricular systole (isovolumetric LV contraction and ejection) and isovolumetric LV relaxation; 2) the conduit phase starts when the $\mathrm{MV}$ opens and is characterized by passive flow from pulmonary veins down a pressure gradient initiated by LV relaxation; and, 3) the active contractile phase (booster pump function), during which the atrium contracts and expels blood into the LV in late diastole, until closure of the MV. 


\section{Components of the mitral valve complex}

Mitral regurgitation occurs during the systolic phase of the cardiac cycle. Because of the complicated structure of the mitral valve (mitral valve complex), and the dynamic interaction of the compounds during the cardiac cycle, the normal mitral valve is described.

The mitral leaflets. Mitral valve closure can be described as the coaptation of the anterior mitral leaflet (AML) and the posterior mitral leaflet (PML), a counter-pressure of the free edges of both leaflets. Both MV leaflets are noticeably different in structure and are referred to as the anterior (aortic) and posterior (mural) leaflets. The PML is small but extends two-thirds around the left atrioventricular junction within the inlet portion of the ventricle. In adults, the PML has indentations (sometimes called "clefts") that generally form three scallops (segments) along the elongated free edge. Usually, these indentations do not extend all the way through the leaflet to the annulus. Carpentier's nomenclature describes the most lateral segment as P1 (adjacent to the anterolateral commissure), P2 is central and the most medial is P3 (adjacent to the posteromedial commissure $)^{13}$. The semicircular AML is much broader than the PML, comprising one third of the annular circumference with a clear and rough zone. The distinguishing feature of this leaflet is the fibrous continuity with the left and noncoronary cusps of the aortic valve and with the interleaflet triangle between the aortic cusps that abuts onto the membranous septum. The AML is also divided arbitrarily into three regions labeled $\mathrm{A} 1, \mathrm{~A} 2$ and $\mathrm{A} 3$ corresponding to the adjacent regions of the PML. From the attachment point of each leaflet at the annulus to the free edge, the leaflet is described as having basal, clear and rough zones. The basal zone is described as the area where the leaflet connects to the atrioventricular junction. The thin central portion of the leaflet is the clear zone. The thick rough zone at the free edge of the leaflet is the main area of chordal attachment and the region of coaptation.

The mitral annulus is a saddle shaped, anatomical inhomogeneous structure with fibrous and muscular portions. The fibrous portion is in continuity with the aortic root and sustains the attachment of the AML from the anterior to the posterior trigone. Contributing to MV closure, three geometrical changes are described in the mitral annulus: the aortic-mitral hinge angle, the mitral annular orifice area and the mitral annular shape.

A. During the isovolumetric contraction of the LV (the pre-ejection phase), the aorticmitral hinge angle increases, leading to a reduction of the septal-lateral mitral orifice and the total mitral area, facilitating the coaptation of the mitral leaflets. Consistent with the mean ventricular pressure curve, the aortic-mitral hinge angle decreases during isovolumetric relaxation, resulting in a minimal angle at the end of diastole ${ }^{45}$. Timek et al. reported that the aortic-mitral flexion increases with enhanced contractility ${ }^{46}$.

B. The mitral valve area changes during the cardiac cycle. In an animal study in 1971 Tsakiris et al. reported annular reduction during pre-systole ${ }^{47}$. These findings 
were confirmed by Ormiston et al., who examined 11 healthy subjects using echocardiography ${ }^{48}$. They measured a maximum mitral area at the end of diastole and a maximum area of reduction throughout the cardiac cycle of $26 \%$. Timek et al. noted that the mitral annular reduction was closely linked to the left atrial dynamics ${ }^{49}$. They found that rapid atrial pacing resulted in a greater mitral annular area and left atrial volume reduction (both entirely during diastole). In a real-time echocardiographic human study, Kwan et al. reported that the annular size increases during systole after presystolic annular narrowing during the isovolumetric LV contraction. The size increases in particular in the anteroposterior direction and reaches a maximum during late diastole ${ }^{50}$. This was consistent with the earlier findings of Pai et $a^{51}$. At that time other studies, in contrast, showed decreased MV area during systole ${ }^{52-54}$, however these results were disputed by Kwan et al. since they were based on calculations of $2 \mathrm{D}$ area projections. It should be noted that whether the mitral annulus contracts or dilates during systole also depends on the definition of LV systole. Since the mitral annulus is the narrowest during isovolumetric LV contraction, it depends on whether systole includes the ejection phase with or without isovolumetric contractions.

C. The mitral annulus is a non-planer and saddle shaped ring with the two highest points situated around the middle of the AML and PML attachment and the lower points at the commissures ${ }^{55}$. Kwan et al. showed that the non-planer shape decreases during systole (becomes flatter), without losing the saddle shape ${ }^{50}$. The rationale for this shape is that the annulus should be able to compensate for the fact that the leaflet length along its attachment remains constant, while the circumference of the annulus changes. Another mechanical consideration is that the saddle shape of the annulus, sustaining the leaflet attachments, acts as a dynamic counter-force to oppose the fast increase of LV pressure during isovolumetric contraction.

The chordae tendineae originate from the tip of the papillary muscles and insert at the mitral valve leaflets at three levels: at the free edge (the primary or marginal chordae), at the leaflet body (the secondary chords, which are thicker than the primary chordae) and at the annular insertion of the leaflets (the tertiary chords - only present at the posterior leaflet). A necropsy study in 100 human hearts shows a unique configuration of chordal anatomy in each heart ${ }^{56}$. Stress and tension on the chordae change during the cardiac cycle in a complex way, which has been extensively studied by Ritchie et al. ${ }^{57}$

The papillary muscles are located anterolaterally and posteromedially in the LV, mostly in the middle third. Variations in PM anatomy are described, supporting 1 to 5 bellies with different forms (conical, flat topped, grooved, etc.), configurations $(\mathrm{V}, \mathrm{Y}, \mathrm{H})$ and arrangements (parallel, interlinked, etc.) ${ }^{56}$. In necropsy studies, the arrangement of the arterial vasculature in the PM was related to the gross morphologic characteristics of the PM: varying between a "fingerlike" PM with one central artery (without 
clear collateral vessels from the adjacent myocardium) and a "sessile" PM with a segmental vascular blood supply from multiple arterial branches penetrating from the adjacent myocardium ${ }^{58}$. Considering the coronary anatomy in three main arteries (the right, the anterior descending and the circumflex coronary artery), the PMs had blood supply from two coronary arteries in 71\% (ALPM) and 37\% (PMPM) and were supplied by a single vessel in 29\% (ALPM) and 63\% (PMPM) ${ }^{59}$. As a clinical consequence, myocardial infarction may cause papillary muscle dysfunction and MR when the blood supply is provided by one rather than two vessels, as is more frequently the case with the posterior rather than the anterior $\mathrm{PM}^{60}$.

The function of the PMs has been well described by Joudinaud et al., by referring to the shock absorbers of the mitral valve complex ${ }^{61}$. During LV contraction, which is a concentric movement and shortening of the LV cavity, the distance between the apical segments of the ventricular wall and base of the heart decreases, as earlier described in the "descent to the base" ${ }^{62}$. At that moment, papillary muscle contraction has to compensate for the reduction of apicobasal LV shortening to maintain the distance from the annulus to the tips of the PMs stable and to prevent leaflet prolapse ${ }^{61}$.

The left ventricle. The contribution of the ventricle to incomplete MV closure is complex and embraces mainly three different, but each influencing, characteristics: 1) left ventricular function which refers to wall motion abnormalities and reduced contractility; 2) left ventricular dimensions which refers to LV dilatation with volume increase; and 3) LV geometrical shape, which mainly refers to the position of the PMs in the LV. The latter can be locally presented by PM displacement or can be globally manifested and expressed as the sphericity of the LV.

\section{The vicious circle of CIMR}

The pathophysiologic background of CIMR is a self-maintaining mechanism leading to heart failure and death. It is clearly explained by the vicious circle that starts with localized or general ischemia and ensues with MR and left ventricular (LV) remodeling. (FIGURE 2)

Acute myocardial infarction (MI) causes LV wall motion abnormalities and reduced contractility (LV function) at one hand and displacement of the papillary muscle(s) away from the MV annulus on the other hand (local LV geometrical changes). The alteration of geometrical relationships between the ventricle and $\mathrm{MV}$ is known as local LV remodeling (local LVR) ${ }^{2}$. Local LV remodeling contributes to MR since increased tension of the chordae tendineae hinder mitral leaflet closure. Chronic volume overload (by impaired LV function with decreased stroke volume), the subsequent increased wall stress and subendocardial ischemia contribute to global left ventricular remodeling $(G L V R)^{63}$. GLVR causes further tethering on the mitral leaflets, which contributes to MR and consequently further LV volume overload and LV dilatation. Ventricular dilatation will viciously amplify the global LV remodeling by increased wall 
stress and subendocardial ischemia. Finally, ventricular function will deteriorate due to the global LV remodeling, leading to heart failure and death.

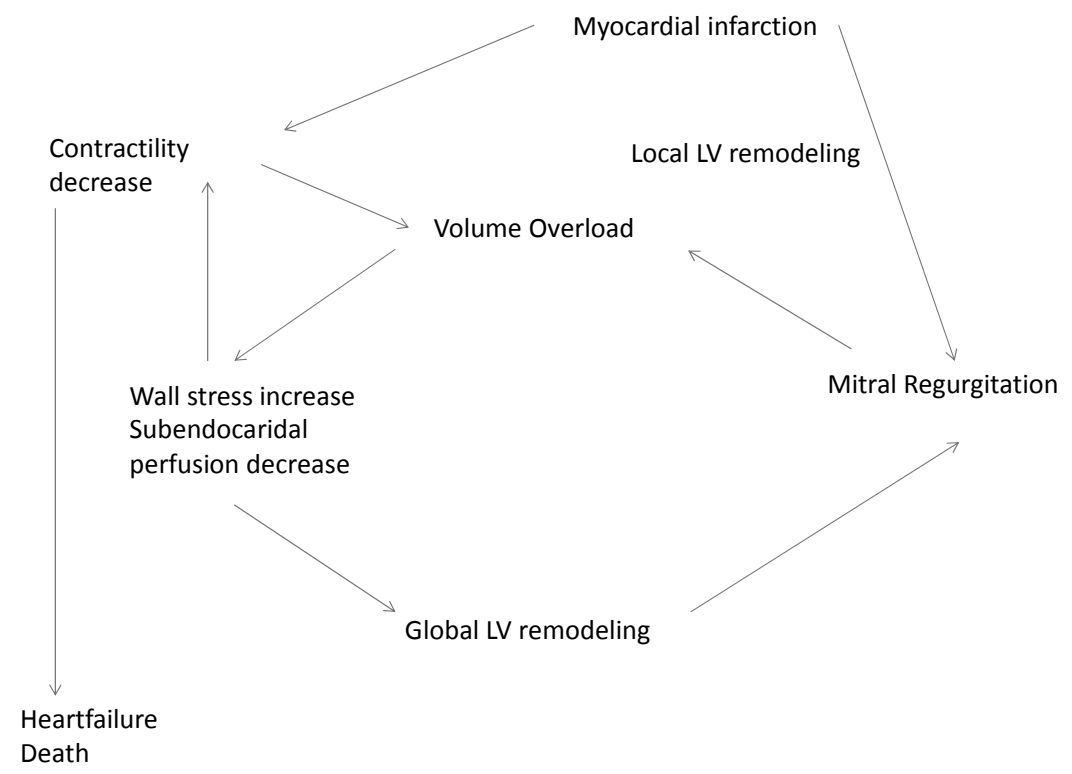

Figure 2. Vicious circle of CIMR

Although CIMR presents with incomplete mitral leaflet closure due to restriction of leaflet motion, it is caused by apical and posterior papillary muscle displacement and wall motion abnormalities in the ischemic ventricle ${ }^{2,64}$. Apparently, the combined muscular revascularization with downsizing of the mitral annulus to restore the mitral leaflet coaptation is not a sufficient therapy to restore the geometrical LV changes in all the patients with CIMR. This finding suggests that we have to differentiate patients with CIMR since not all the valves can be restored by restrictive annuloplasty. This is proven by the fact that MR in ischemic MR is a disease of the ventricle, not of the valve itself.

\section{Historical notes in understanding the mechanism of ischemic mitral insufficiency}

In 1963, Burch et al. proposed a possible role for papillary muscle dysfunction in the etiology of IMR ${ }^{65}$. They suggested that failure of papillary muscle shortening in systole, due to sequelae of ischemia, aborted the counter- tension on the mitral leaflets during the systolic LV closing forces (isovolumetric LV contraction). MR was caused by bulging of the leaflets (prolapse) into the left atrium. However, experimental studies have shown that isolated anterior or posterior papillary muscle dysfunction or combined 
papillary muscle dysfunction does not cause MR in a normal contracting left ventricle ${ }^{66}$, 67. This observation was confirmed by Mittal et al. who reported a dog experiment showing that MR only occurred when the papillary muscle and adjacent LV wall where injured $^{68}$. Burch et al. also addressed the theory that dysfunction of the LV wall was involved in creating mitral regurgitation ${ }^{65,69}$. In this model, they alternatively postulated systolic restriction of the mitral leaflet motion. Normally, the distance between the papillary muscle tips and the mitral annulus remains stable throughout the cardiac cycle, while considerable dynamic changes occur in the distance between the apex and mitral annulus ${ }^{61}$. Burch et al. reported that the ischemic aneurysmatic deformation of the LV wall, adjacent to the papillary muscles, causes a posterior and outward displacement of PMs in relation to the MV. By increasing the distance between the papillary muscle tips and mitral annulus, the mitral leaflets were drawn into the LV, restricting their motion towards closure ${ }^{65,69}$. Interesting in this respect was the experimental finding of Mittal et al., that injury to the PM (by ethanol injection) did not produce acute MR, but that MR occurred in the chronic phase with PM scarring and retraction $^{68}$. This study suggests that ischemic MR, without PM rupture, does not appear acutely, but later when muscular scarring inhibits muscle contraction. Later, Jouan et al. observed that in occasional patients with $\mathrm{MI}$, mitral leaflet prolapse was found based on PM anatomy, resembling PM elongation ${ }^{70}$. They reported that incomplete PM rupture could mimic PM elongation. Whether the PM elongation represented a late sequel of incomplete PM rupture or whether it could be a primary lesion due to a distension of a scarred PM submitted to excess tension remains debatable.

Godley at al. introduced the terminology of "incomplete mitral leaflet closure" (IMLC), based on a two-dimensional echocardiographic study in post infarct patients in whom they observed MR as a result of mitral leaflet tethering ${ }^{71}$. They found that patients with IMLC demonstrated dyskinetic wall motion in the region immediately surrounding one of the papillary muscles and that the posteromedial papillary muscle was primarily affected.

In a later study on dysfunction of the PM, Kisanuki et al. investigated the fractional shortening between end-diastolic PM length and end-systolic PM length by 2D echocardiocardiographic imaging from an apical view (longitudinal visualization of the $P M s)^{72}$. The authors found that in patients with prior myocardial infarctions, a significant decrease in fractional shortening was observed in proportion to the severity of left ventricular wall motion abnormalities at the site of papillary muscle implantation. Moderate or severe mitral regurgitation was significantly more frequent in patients with combined anterior and posterior papillary muscle dysfunction than in those with isolated anterior or posterior dysfunction or with normal function of both papillary muscles. This study already suggested the idea that ischemic MR has a higher incidence in patients with a more extended ischemic involvement of the left ventricle than in patients with isolated regional infarctions. 
In a canine experiment Kaul et al. reported that neither reducing PM arterial perfusion nor reducing adjacent LV wall perfusion - nor the combination of both - produced MR. In contrast, global LV hypoperfusion, despite continued selective PM arterial perfusion with visible thickening and intact adjacent LV wall, caused MR. The severity of MR was directly correlated with global LV dysfunction ${ }^{73}$. This theory was confirmed by Dent et al. who reported that the extent of mitral leaflet excursion was determined by global LV function and not by papillary muscle dysfunction ${ }^{74}$.

However, in an in vivo canine model, Otsuji et al. failed to demonstrate MR by creating pharmacologically global LV contractile dysfunction (ejection fraction <20\%) without LV dilatation (LV expansion was limited through pericardial restraint). By opening the pericardium, moderate MR was created, accompanying significant LV dilatation. Mitral regurgitant volume and orifice area did not correlate with LV ejection fraction and $\mathrm{dP} / \mathrm{dt}$ (global function) but did correlate with changes in the distance of the $\mathrm{PM}$ tips to mitral annulus as the only independent predictor of $\mathrm{MR}^{75}$.

The existence of action (LV contraction) and counter-action (PM contraction) to stabilize the tethering length (distance from PM tips to the mitral annulus) seems to be indispensable to obtain MV closure. This explains the experimental findings of Messas et al. showing that MR, created by a limited inferobasal MI and LV wall bulging, disappeared by making the adjacent PM acutely ischemic, causing passive, "paralytic" PM elongation in response to ventricular closing forces ${ }^{76}$. This study was confirmed by Khankirawatana et al. who demonstrated a strong correlation between preserved PM contraction (by tissue Doppler imaging) and MR (increased leaflet tenting) in patients with diminished LV contraction ${ }^{77}$.

Proceeding with the findings of Kaul et al. and Dent et al. ${ }^{73,74}$, correlating MR to global LV dysfunction, $\mathrm{He}$ at al. described an in vitro model of excised porcine mitral valves to distinguish LV contractile dysfunction (function) from LV dilatation (dimensions and geometry) in creating functional $\mathrm{MR}^{78}$. By changing the geometry of the mitral leaflet attachments in a direction that would increase leaflet tension (at both the papillary muscle and annular ends of the mitral orifice) they were able to cause functional mitral regurgitation. On the other hand, they demonstrated a variation of the regurgitant orifice area depending on the transmitral pressure, generated by ventricular contraction (LV function), even though the papillary muscle and annular geometry were preset in each stage of the study ${ }^{78}$. This study emphasizes the competing nature of tethering forces opposing coaptation and the transmitral pressure promoting it (the balance of forces). Besides dilatation, LV geometry was further defined by sphericity of the LV, with a potential displacement of the PMs posteriorly and laterally, which was confirmed by 3D echocardiography ${ }^{75}$. Several authors have correlated MR with LV sphericity ${ }^{79-81}$. In a later study, presented by Yiu et al., valvular tenting was addressed to local LV remodeling (apical and posterior displacement of PMs) and was independent of global LV remodeling ${ }^{2}$. 
Localized remodeling of LV segments was the interest of further research. After the reports of clinical improvement of heart failure (HF) by cardiac resynchronization therapy (CRT) in patients with HF and left bundle branch block (LBBB) ${ }^{82,83}$, the effect on MR was investigated. Kanzaki et al. reported that CRT significantly and immediately reduced MR. They contributed this success to the improved coordinated timing of mechanical activation of the PM sites ${ }^{43}$. This finding concentrated further research on the dyssynchrony between the activation of the PM in patients with CIMR, and has become the basis of the studies presented in this thesis.

\section{Contribution of individual components of the mitral valve complex to CIMR}

MR occurs during the systolic phase of the cardiac cycle. Because of the complicated structure of the mitral valve (mitral valve complex), the dynamic interaction of the compounds during systole will be discussed and pathophysiological changes resulting in CIMR will be elucidated.

The mitral leaflets

In patients with CIMR, the leaflets of the mitral valve are structurally not involved in the etiology of $M R$, but their function during systole (leaflet coaptation) is hindered by tethering forces.

\section{The mitral annulus}

Contributing to MV closure, three geometrical changes are described in the mitral annulus: the aortic-mitral hinge angle, the mitral annular orifice area and the mitral annular shape.

A) The aortic-mitral flexion increases with enhanced contractility ${ }^{46}$. As a consequence, in patients with ischemic heart disease and impaired LV contractile function, this preset condition of proper mitral valve closure is hampered.

B) In functional MR, the mitral annulus dilates and shows lack of area reduc$\operatorname{tion}^{52,84,85}$. Although annular dilatation clearly contributes to failure of leaflet coaptation, it is not the dilation itself that causes MR. In a comparison of two patient groups with similar dilation of the mitral annulus, but with different pathological substrate (lone atrial fibrillation and dilated cardiomyopathy), MR was only present in case of LV dilatation ${ }^{86}$. Because of the anatomic structure of the mitral annulus, it was believed that annulus dilatation was solitarily restricted to the posterior, muscular, annulus and not present in the fibrous part. Conversely, Ahmad et al. found that in IMR patients, annular dilatation was mainly caused by an increase in anterior and posterior annular perimeters, which was accompanied by an increase in the intertrigonal distance and a restriction of annular motion ${ }^{84}$. Similar observations were made by Watanabe et $\mathrm{al}^{87}$. In a chronic ischemic sheep model, Tibayan et al. were able to evoke dilation of the fibrous annular part ${ }^{85}$. 
C) Several authors report that in patients with IMR, the saddle shape of the mitral annulus flattens ${ }^{84,87}$. It is unclear whether this evolution is caused by annular or ventricular dilatation. It is clear that by losing the saddle shape of the mitral valve, MR will progress. This is the reason why several authors suggest non-planer annuloplasty rings for mitral valve repair in $\mathrm{IMR}^{87}$.

Some authors describe a fourth geometrical change of the mitral annulus, apicobasal annular motion ${ }^{52,88}$, in accordance with "descent to the base" (DB), which was first described by Simonson et al. to quantify left ventricular function ${ }^{62}$. DB describes a systolic excursion of the mitral annulus to the apex, which decreases when left ventricular function deteriorates. The apex is nearly stationary, moving a few millimeters in the same direction as the base. In healthy individuals the descent of the base is 1.0 to $1.5 \mathrm{~cm}$. Similar findings are shown for apicobasal motion measurements, with the largest atrial excursion of the mitral annulus during the pre-ejection phase. It is evident that apicobasal motion is a reproduction of LV function, saddle shape motion and hinge angle variation. It is at the end of the isovolumetric LV contraction that the mitral valve has the smallest annulus, the highest aortic-mitral hinge angle, the most distinct saddle shape and highest position into the left atrium.

\section{The chordae tendineae}

Studying the function of the chordae tendineae in IMR, Messas et al. reported in an ischemic animal study, a reduction of MR by cutting the secondary chordae of the AML without causing prolapse ${ }^{89}$. In a CIMR study, the same group reported in a chronic follow-up study a reduction of MR and decreased progression of global LV remodeling (stable EF but declined increase of LV volumes compared to controls) by cutting secondary chordae at AML or both leaflets ${ }^{90}$. These results conflictied with the earlier findings of Rodriguez et al., demonstrating a decline in LV function following secondorder chordal cutting ${ }^{91,92}$. However, the latter authors studied the interaction between the second-order chordae of the AML and the LV geometry and function in an acute ischemic open chest sheep model; chordal cutting was performed off-pump by passing an electrocautery radiofrequency current through a wire which was pre experimental operatively encircled around the second-order chordae and exteriorized through the LV wall. The study limitation in this small animal group $(n=8)$ was that changes in LV function could have been caused by collateral damage to the endocardial LV surface from the electrocautery current or perhaps mechanical "tugging" on the second-order chordae during transection. Moreover, in this off-pump model, chordal cutting was not always restricted to the second-order chordae, but primary chordae were accidentally involved. The concern of chordal presence originated from the studies on the physiological function of the second-order chordae. In porcine studies, van Rijk-Zwikker et al. $^{93}$ found that the AML second-order chordae remained taut during the cardiac cycle and Lomholt et al. ${ }^{94}$ reported that the forces on the second-order chordae were 3-fold higher than those on the first-order chordae $(0.7 \mathrm{~N}$ versus $0.2 \mathrm{~N})$. 


\section{The papillary muscles}

Tanimoto et al. studied the prevalence of papillary muscle infarction (by early and late gadolinium-enhanced MRI) in patients with acute $\mathrm{MI}$ and correlated these data with the coronary lesions (by coronary angiography) and the occurrence and severity of MR (by echocardiography) ${ }^{95}$. In $40 \%$ of the patients PM infarction was diagnosed: $77 \%$ in the PMPM and $26 \%$ in the ALPM. PM infarction was encountered significantly more frequent in patients with left circumflex (78\%) and right coronary artery (48\%) lesions compared with left anterior descending artery lesions (13\%). Patients with PMPM infarction presented more frequently with MR than patients with ALPM infarction. However, the presence of PM infarction was not associated with the presence and severity of MR in the acute phase and based on the late MRI Tanimoto et al. concluded that it was the size of the myocardial infarction (and not the presence of PM infarction) that affected the LV remodeling (defined as increased LV EDV > 15\%) and thus MR. The limitation of this study was the follow-up time of 8 months, and the long-term data on progressive local LV remodeling are not available.

Messas et al. reported in an experimental ovine study the paradoxical reduction of MR, which was initially created by a limited inferobasal myocardial infarction with local LV wall bulging, by inducing ischemia to the adjacent $\mathrm{PM}^{76}$. Uemura et al. confirmed these findings in a clinical setting. They reported an attenuation of ischemic MR due to PMPM dysfunction (by strain rate imaging) in patients with localized basal inferior LV remodeling ${ }^{96}$.

Recent studies have demonstrated the beneficial effect of cardiac resynchronization therapy (CRT), showing the immediate reduction of functional mitral regurgitation $(\mathrm{FMR})^{43,97,98}$, due to the improved coordinated timing of mechanical activation of PM insertion sites and the remote decrease of FMR secondary to LV reverse remodeling $^{43}$. This can be understood since dyssynchrony causes an uncoordinated regional LV mechanical activation, altering uncoordinated mitral leaflet motion and tethering. Moreover, the improper timing of the AV relaxation and contraction cycle causes a positive pressure gradient between the left atrium and left ventricle, which effects MV closure. Finally, dyssynchrony will decrease LV contraction efficiency, causing lower closing forces and supporting mitral leaflet tenting ${ }^{99}$. Papillary muscle systolic dyssynchrony and IMR will be discussed in Chapter 4 in relation to the recurrence of $\mathrm{MR}^{100}$, in Chapter 5 as a predictor of recurrent MR in patients with ischemic dilated cardiomyopathy and severely impaired LV function ${ }^{101}$ and in Chapter 6 in relation to the symmetric tethering pattern of the mitral valve ${ }^{102}$.

\section{The left ventricle}

The contribution of the ventricle to incomplete MV closure is complex and embraces mainly three different, but each influencing, characteristics: 1) left ventricular function; 2) left ventricular dimensions; and 3) LV geometrical shape (locally or globally). 
Obviously, these ventricular determinants do not have an independent relationship to themselves ${ }^{2}$ as clearly described in the section on the pathophysiology of CIMR and the vicious circle in this thesis.

In vitro and animal studies have aimed to differentiate between the ventricular determinants and their contribution to $\mathrm{MR}^{78,103,104}$. To distinguish LV geometry from function, He et al concluded that geometrical change - by displacing the papillary muscles of excised porcine mitral valves (in vitro) in a direction that would increase leaflet tension - was sufficient to create MR. The contribution of LV function was shown since they could measure a variation of regurgitant orifice area depending on the exposition of the valve to alternating transmitral pressure (mimicking LV function), despite the preset papillary muscle and annular geometry in each stage of the study ${ }^{78}$. Liel-Cohen et al. ${ }^{104}$ tested the impact of local LV remodeling in a chronic ischemic sheep model by ligation of the circumflex artery with consequent displacement of PM posteriorly and inferolaterally and the occurrence of MR by tethering of the leaflets. They reduced MR by off-pump plication of the bulging infarction region, while LV function, global endsystolic volume and mitral area remained stable. They concluded that the only independent predictor for MR was the tethering distance of the PM to the annulus ${ }^{104}$. Tenting was particularly determined by outward movement of the papillary muscle that was well demonstrated in both the acute and chronic ischemic models of Otsuji et al. ${ }^{75,}$ 105 Finally, Kono et al. created a canine animal model to test the impact of local LV geometrics $^{103}$ and global LV remodeling ${ }^{79}$ on the occurrence of ischemic MR. After creating acute local myocardial ischemia by injecting microspheres in the circumflex artery, acute MR occurred which completely subsided within 3 weeks. The temporary hypokinesia of the ventricular segment overlying the papillary muscle, leading to retraction of the mitral leaflets toward the apex, appeared to be a sufficient condition for incomplete leaflet coaptation ${ }^{103}$. Subsequently, the same group created a canine chronic heart failure model (by alternately injecting microspheres in the anterior descending and circumflex coronary artery). After 12 weeks, dogs developed MR, preceded by increased sphericity of the left ventricle. Remarkably, the other determinants contributing to MR (LV volumes, mitral annulus dilatation and wall motion abnormalities) occurred later in time, demonstrating that global LV remodeling is the primary cause of functional mitral insufficiency in chronic ischemic myocardial disease ${ }^{79}$.

Reports on the association between mitral regurgitation and the location of myocardial infarction are conflicting. While some have reported that MR was associated with inferior infarction ${ }^{60}$, other studies reported a higher prevalence of MR in anterior myocardial infarction ${ }^{106}$ or showed no difference. Perhaps the higher prevalence in anterior infarctions occurred in those with larger areas of ischemia and subsequently global dysfunction. This can be abstracted from the difference in anatomical blood supply to both PMs, where the ALPM is mostly supplied by two vessels, with the consequent need of larger ischemic regions to cause ALPM dysfunction and MR, while on the contrary the PMPM is mostly supplied by one vessel and is more at risk for ischem- 
ic injury. However, Lehmann et al. did not observe an association between MR and conventional biochemical indicators of severity of $\mathrm{MI}^{106}$.

The progression of LV remodeling or the occurrence of LV reversed remodeling in CIMR patients after coronary bypass grafting and restrictive annuloplasty, is discussed in this thesis in the section on outcome after CABG and UMRA.

\section{Echocardiographic evaluation of CIMR}

Historically, the severity of MR was assessed by left ventriculography, relying on the retrograde opacification of the blood flow into the left atrium during systole ${ }^{29,30}$, but this technique did not provide adequate information on the anatomical and functional structures of the MV complex, nor could it enlighten the etiology of MR. Since the severity of MR in patients with CIMR has implications for their prognosis and treatment and since the etiology of CIMR is an ischemic myocardial disease of the LV, it is important to provide 1) quantitative information of MR, 2) geometrical measurements of the MV and the LV and 3) functional and dimensional LV measurements. Echocardiography is currently the preferred method to assess valvular heart disease ${ }^{107,108}$. Recently, also cardiac magnetic resonance imaging (MRI) is increasingly used in ischemic patients for the evaluation of the ventricular dimensions and function and the visualization of scarring ${ }^{109}$. Contrast-enhanced MRI, showing scar transmurality, can predict the likelihood of myocardial functional recovery (by segmental contractility) following revascularization. Kim et al. showed that myocardial contractile recovery after revascularization was present in $78 \%$ of the myocardial segments in case of absence of scar tissue, and in less than $2 \%$ of the segments when scar tissue was more than $75 \%$ transmural (gadolinium hyperenhancement) ${ }^{110}$. However, viability of myocardial tissue is as such not the predicting parameter of functional myocardial recovery. Bax et al. reported that extensive LV remodeling did not allow the viable myocardium to improve in LV function after revascularization, and that this situation was associated with a poorer long-term prognosis ${ }^{111}$. The relative disadvantage of cardiac MRI in clinical practice is the limited availability and fact that the investigation is time-consuming and contraindicated in claustrophobic patients or in patients with pacemakers or defibrillator and resynchronization therapy devices. Since this thesis was based on retrospective studies of patients, MRI was not performed and the dimension of muscular scarring and viability was not available. 


\section{The mitral valve}

\section{Evaluation of $M R$}

Ischemic MR is a dynamic phenomenon. Several studies described a decrease of MR severity in patients under general anesthesia, probably due to the drop in systemic vascular resistance caused by the anesthetic agents ${ }^{112-114}$. Therefore, it is recommended that the severity of MR be measured and the surgical strategy determined before anesthesia.

However, Dion et al. introduced a peroperative dynamic test to evaluate if an increased MR could be evoked, which should justify MV repair in ischemic patients ${ }^{115}$. First they increased the preload (preload test) by rapid fluid filling through the arterial cannula, increasing the pulmonary wedge pressure. Secondly (if the preload test was negative) they increased the afterload (afterload test) by administering a $5 \mathrm{mg}$ IV bolus of phenylephrine, increasing vascular resistance (without inotropic effects). If MR did not increase, MV repair was not performed. Afterwards, several authors reported a variation of dynamic tests to evaluate the severity of MR for surgical strategy ${ }^{116-118}$.

Standard color Doppler imaging is a highly sensitive method to detect even mild degrees of IMR. The color flow mapping of the regurgitant jet area compared to the left atrial area has been used for years as a semi-quantitative evaluation of $M R^{119}$. To quantify the severity of MR, the proximal isovelocity surface area (PISA) and vena contracta method is used, analyzing the proximal flow convergence to calculate the effective regurgitant orifice area (EROA or ERO), the regurgitant volume (RV) and regurgitant fraction $(\mathrm{RF})^{120}$. The vena contracta is defined as the narrowest part of the regurgitant jet recorded in the parasternal long-axis view. The ERO indicates the severity of the lesion, while the RV explains the volume overload. The current guidelines recommend thresholds to define severe mitral regurgitation as a $\mathrm{RV} \geq 60 \mathrm{ml}$, a $\mathrm{RF} \geq 50 \%$ and an $E R O \geq 40 \mathrm{~mm}^{2}{ }^{121}$. However, these criteria were adapted for ischemic mitral regurgitation since adverse outcome was associated with lower values of these parameters, suggesting $30 \mathrm{ml}$ for $\mathrm{RV}$ and $20 \mathrm{~mm}^{2}$ for $\mathrm{ERO}^{1}$. This can be explained since the IMR has a dynamic nature: small amounts of regurgitation at rest can increase to severe $M R$ during exercise. Lancelotti et al. reported that the exercise induced increase of ERO was related to local remodeling (rather than to global remodeling), more specifically to papillary muscle displacement, leading to increased leaflet tethering ${ }^{122}$. The same authors reported that an exercise-induced increase of ERO with $>13 \mathrm{~mm}^{2}$, was associated with increases in pulmonary pressure, pulmonary edema and cardiac death ${ }^{123,124}$.

\section{Geometrical leaflet measurements}

Further assessment of MR is based on the geometrical relationship of the mitral leaflets and the annular plane during the systole. MR is caused by loss of leaflet coaptation since in CIMR leaflets are tethered into the ventricle. Geometrical leaflet parameters are usually assessed at mid-systole by transthoracic echocardiography (TTE) in the 
parasternal long-axis view or by transoesophageal echocardiography (TEE) at the A2-P2 level. In Figure 3 the geometrical leaflet parameters are shown.

\section{Mid-Systole}

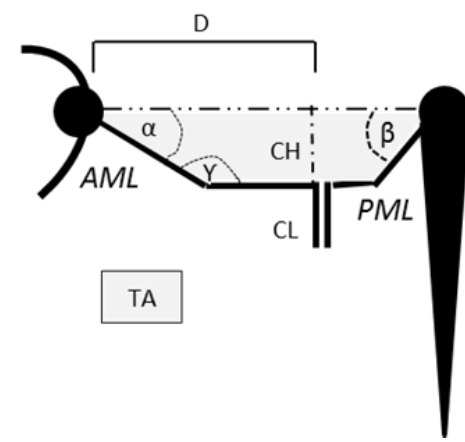

\section{Mid-Diastole}

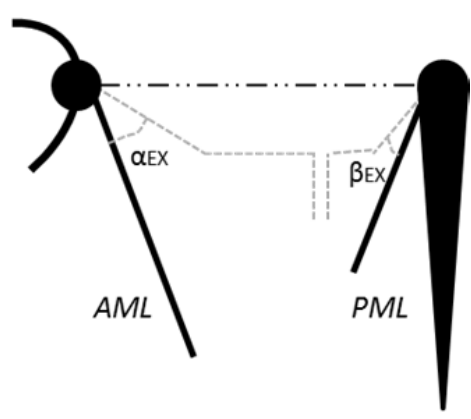

Figure 3. direct measurements in the parasternal long-axis view in mid-systole:

TA = tenting area: area enclosed between the annular line and the mitral valve leaflets

$\mathrm{D}=$ coaptation distance: distance between anterior annulus and coaptation-line

$\mathrm{CH}$ = coaptation height: distance between annular plane and start of coaptation

$\mathrm{CL}=$ coaptation length: length of coaptation

$\alpha=A M L$ tethering angle: angle between annular plane and base of AML

$\beta=P M L$ tethering angle: angle between annular plane and base of PML

$\alpha / \beta=$ tethering pattern: the closer to 1 , the more symmetric the tethering pattern is

$Y=$ bending angle: angle between the line from the anterior annulus to the bending point of $A M L$ and the line from the bending point to the coaptation point

$\alpha_{\mathrm{EX}}=\mathrm{AML}$ excursion angle: angle between the base of AML at mid-systole and mid-diastole

$\beta_{\mathrm{EX}}=\mathrm{PML}$ excursion angle: angle between the base of PML at mid-systole and mid-diastole

These parameters have been used to specify surgical repair techniques. Dion et al. promoted a thoroughly downsizing of the mitral annulus by a restrictive annuloplasty ring, resulting in a coaptation length $>8 \mathrm{~mm}$ and no residual MR to obtain a good long-term follow up ${ }^{125}$.

Recently, attention has been drawn to preoperative leaflet configuration to predict MR recurrence and outcomes. However, published data are conflicting: recurrence of $M R$ was associated with preoperative tethering of the posterior mitral leaflet ${ }^{126,127}$ or tethering of both leaflets ${ }^{128}$. The importance of the tethering pattern $(\alpha / \beta)$ contributing to $M R$ in ischemic patients was demonstrated by Agricola et al ${ }^{129}$. The association of preoperative AML tethering and the recurrence of MR was shown by Gelsomino et $\mathrm{al}^{130}$. In this thesis the importance of the tethering of the AML as an independent predictor for recurrence of MR will be explained (Chapters 2 and 3). 


\section{The left ventricle}

To evaluate CIMR, assessment of LV volumes and geometry are inevitable since MR is caused by the ischemic disease of the LV. The LV condition can be assessed by measuring volumes, defining the geometry and determining the function. The measurement of LV diameters (end-systolic and end-diastolic) has been performed by several authors $^{131,132}$. However, one can conclude that this two-dimensional evaluation of the ventricular cavity is less accurate than the volume calculation, certainly in the presence of LV aneurysms or enlarged left ventricles ${ }^{133}$. Echocardiographic technical details of measuring the LV parameters, are outside the scope of this thesis.

\section{$L V$ volumes}

End diastolic $L V$ volume (LV EDV) and end systolic LV volume (LV ESV) are measured, as described by Schiller et $\mathrm{al}^{134}$. Regarding predictive factors for recurrence of MR after restrictive annuloplasty, Gelsomino et al. reported a LV ESV $\geq 145 \mathrm{ml}$ as an independent predictor ${ }^{4}$. Based on the LV ESV after mitral valve repair compared to baseline measurements, Stellbrink et al. considered that a decrease of $>15 \%$ defined left ventricular reversed remodeling (LVRR) ${ }^{135}$.

\section{LV geometrical assessment}

The remodeling of the left ventricle (LVR) is described by the geometrical changes and can be evaluated globally or locally. For global evaluation of the cavity, the sphericity indexes are used. These indexes are obtained at the end of the diastole $\left(S I_{D}\right)$ and at the end of the systole $\left(S I_{S}\right)$ as the volume of the LV divided by the volume of a sphere with a diameter equal to the longest axis of the LV, measured in the apical view ${ }^{79}$. The more this number approaches 1 , the more the LV is shaped as a sphere. Kono et al. showed in an ischemic canine model that the onset of MR was correlated with the sphericity of the $\mathrm{LV}^{79}$. In ischemic patients the SIS $\geq 0.7$ was an independent predictor of recurrence of MR after $\mathrm{RA}^{4}$. To evaluate the local geometrical changes, the lateral and inferior displacements of ALPM and PMPM can be quantified as the distance between the PM head and well-defined anatomic landmarks at early and end systole from the parasternal short-axis view ${ }^{2,129}$. The separation between both papillary muscles can be measured directly and the apical displacement of the PMPM can be measured on the long-axis view as the distance between the PMPM head and the fixed inter-valvular fibrosa (insertion of $\mathrm{AML}$ ).

\section{$L V$ function}

Left ventricular ejection fraction ( $L V E F)$ can be assessed by the bi-apical Simpson disk method $^{134}$ but since this calculation is derived from the LV volumes, it remains dependent on imaging quality and geometrical changes. To overcome this bias, the LV function can also be quantified by the myocardial performance index (MPI), calculated 
by the ratio of the time intervals: the sum of the isovolumetric LV contraction and relaxation time interval, divided by the LV ejection time interval ${ }^{136}$. The MPI has been shown to be an independent predictor of $M R$, with a cut off $\geq 0.9$, after RA in patients with $\mathrm{CIMR}^{4}$. This measurement not only reflects systolic myocardial function, but evaluates systolic and diastolic function in combination. Alternatively, systolic LV function can be obtained from the wall motion score index (WMSI), which is derived by grading the wall motion of individual myocardial segments and dividing the total score by the number of the analyzed segments ${ }^{137}$. WMSI has been reported as a prognostic assessment of mortality for patients after acute myocardial infarction ${ }^{137,138}$. Gelsomino et al. reported that WMSI $\geq 1.5$ was an independent predictor of recurrence of $\mathrm{MR}^{4}$ when segmental LV evaluation was performed according to the 17 -segment model ${ }^{139}$. Finally, the LV function can be described by the global $\boldsymbol{L} \boldsymbol{V}$ strain. Strain is the measurement of relative tissue deformation as a result of applied stress. The mathematical concept of myocardial strain was first formulated in 1973 by Mirsky et al. ${ }^{140}$. It represents the percentage of the original unstressed tissue dimension and includes lengthening or expansion of tissue (positive strain) and shortening or compression of tissue (negative strain). For myocardial strain, the change in tissue dimension is relative and compared to the basic dimension, which is mostly the initial muscle length at the end of diastole. The advantage of strain measurement is that it quantifies the real myocardial contraction potential which is not clearly produced by the LV EF. E.g. in hypertensive patients with a normal LV EF, the myocardial fiber shortening can be seriously depressed ${ }^{141}$. Moreover, it has been demonstrated that strain was lower in patients with asymptomatic MR and normal LV ejection fraction, indicating subclinical LV dysfunction ${ }^{142}$.

Initially, strain was measured by tissue Doppler imaging (TDI), which is a velocitybased technique ${ }^{143,144}$, and validated as a new method to quantify regional myocardial function $^{143}$. Strain rate is defined as the temporal derivative of strain or the tissue deformation rate ${ }^{145}$. The principal limitation of TDI is the dependence on the Doppler angle of interrogation, resulting in a primarily assessment of longitudinal strain. However, the left ventricular systolic ejection is a coordinated action involving fiber shortening in multiple directions ${ }^{146,147}$ as well as systolic torsion ${ }^{148,149}$. These actions produce fiber shortening in a longitudinal and circumferential direction and wall thickening in a radial direction ${ }^{150}$. However, Hurlburt et al. observed a significant regional heterogeneity in the circumferential strain that was not present in the longitudinal and radial strain ${ }^{150}$. To investigate the three dimensions of strain, MRI measurements were performed $^{151,152}$, but the complexity of this application is limited in clinical routine.

Echocardiographic two dimensional strain imaging, in contrast to TDI strain, is based on B-mode ultrasound signal intensity and is angle independent ${ }^{153-155}$. The twodimensional speckle tracking is a custom acoustic-tracking software that allows semiautomated strain analysis. It utilizes B-mode gray-scale images and tracks movement of stable acoustic patterns/markers, called speckles, in the myocardial tissue. This speckle tracking takes place frame by frame throughout the cardiac cycle ${ }^{155}$ 
and allows differentiation of myocardial segments with active contraction from segments which are passively tethered. Measuring the strain of the LV, the longitudinal strain seems to be the most homogenous ${ }^{150}$ and the important contribution of the longitudinal contraction for LV function has been put forward by several authors ${ }^{62,146}$. For global longitudinal LV strain (GLS) measurement, digital loops are acquired from apical 2-,3- or 4-chamber views. The software is interatcive, in that the endocardialcavity interface is traced manually in one image; two additional (mid and outer) lines are produced by the software to define the region of interest. For each view, the software automatically divides the ventricle into six segments and provides an automated tracking of the acoustic markers (speckels) throughout the cardiac cycle in each segment. For each view, a curve of global strain is obtained per segment and an average of these curves is generated. The peak global longitudinal systolic strain (GLS) is the average of the peaks of these average curves, obtained in each view. (Figure 4) For healthy individuals, the peak global longitudinal systolic strain is $-18.6 \pm 0.1 \%{ }^{156}$. Since longitudinal strain of the LV is charcaterized by fiber shortening, the GLS is a negative number. GLS was validated by Brown et al. as an investigation of LV function ${ }^{157}$. In an animal study, Carlhäll et al. revealed that transmural strain may be an early marker of LV dysfunction after development of $M{ }^{158}$. Lancellotti et al. demonstrated that longitudinal speckle-tracking-derived strain had impaired values in patients with asymptomatic MR and normal LV ejection fraction, indicating subclinical dysfunction $^{142}$.

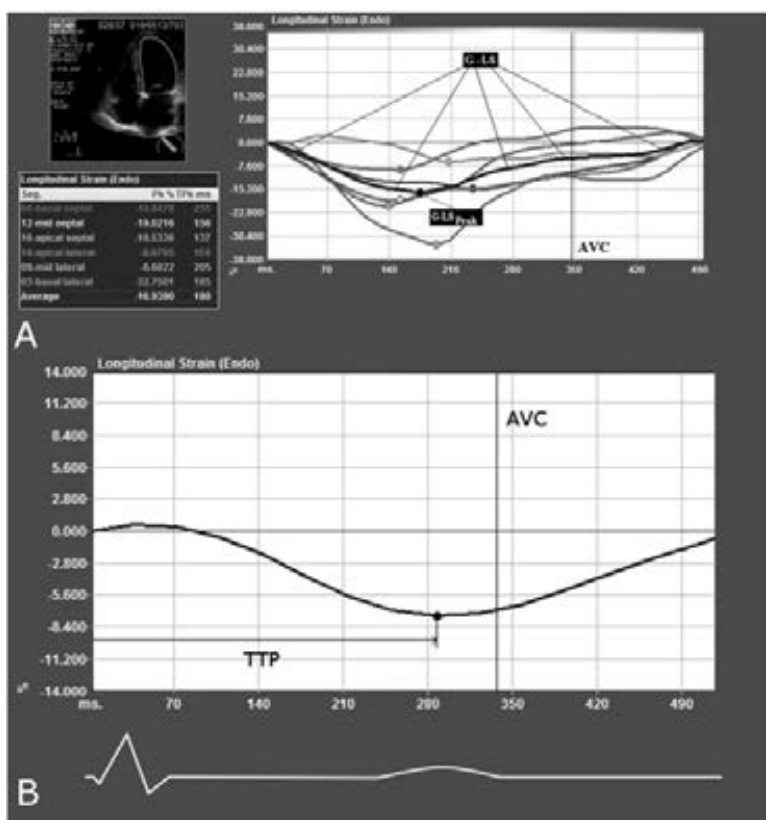

Figure 4 A. Representative example of measuring Global Longitudinal Strain (G-LS) and Global Longitudinal Strain peak $\left(\mathrm{G}-\mathrm{LS}_{\text {peak }}\right)$ in an apical 4-chamber view. Abbreviations: AVC: Aortic valve closure.

B. Representative example of measuring time to peak (TTP) of the $\mathrm{G}-\mathrm{LS}_{\text {peak}}$, beginning at the start of the QRS-complex. Abbreviations: AVC: Aortic valve closure. 
Diastolic filling patterns of $L V$

Mitral inflow velocities are measured by pulsed wave Doppler (PW-Doppler). In persons with sinus rhythm two velocity curves can be recorded during diastole: the $\boldsymbol{E}$ wave, representing the early, passive filling of the left ventricle ( $E=$ peak early transmitral flow velocity), and the $\boldsymbol{A}$-wave, which occurs late in diastole, representing active LV filling and atrial contraction ( $A=$ peak late transmitral flow velocity). Also the $E / A$ ratio and the deceleration time of $\boldsymbol{E}$ velocity (DT) can be obtained by PW-Doppler ${ }^{159}$. The isovolumetric relaxation time (IVRT) can be obtained from the apical five-chamber view across the region between the aortic outflow tract and the mitral inflow tract. Several studies have shown an association between abnormalities of LV relaxation and specific transmitral Doppler flow velocity patterns. In ischemic patients Doppler signals exhibit a pattern of "delayed relaxation", characterized by decreased early filling (E) and increased atrial contraction (A) mitral flow velocities. Impairment of LV relaxation results in prolongation of the isovolumetric relaxation time, a decrease in $E$ and a prolongation of the $\mathrm{E}$-wave $\mathrm{DT}^{160}$. However, other factors such as left atrial pressure can influence transmitral Doppler flow ${ }^{161}$. Progressive elevation of LA pressure in patients with impaired LV relaxation results in "pseudo-normalization" of the filling pattern with a decreased $\mathrm{DT}^{162}$. To overcome this limitation of transmitral flow velocity measurements, the pulmonary venous flow (PV) and thus systolic-to-diastolic ratio S/D are routinely assessed ${ }^{163}$. Most normal adult patients exhibit a prominent systolic (S) flow and a systolic-to-diastolic (S/D) ratio $>1$. In patients with elevated LV filling pressure, reduced LA and LV compliance or with severe $M R$, there is blunting of the pulmonary venous $S$ wave and an increased $D$ flow. This pattern, in addition to prominent atrial reversal $(A R)$ flow velocity, has been used to distinguish normal from pseudo-normal transmitral Doppler filling ${ }^{162}$. In patients with CIMR DT $<140 \mathrm{~ms}$ and S/D $<0.8$ they have been shown to be independent predictors of early and late death ${ }^{164}$. In this study, DT $<140$ ms was the only diastolic independent predictor of MR recurrence. In this thesis atrial function in patients with CIMR before and after CABG and MV repair by restrictive annuloplasty were investigated by left atrial strain and strain rate measurement by 2D-speckle tracking echocardiography (Chapter 8 ). The evaluation of the left atrial function by atrial strain and strain rate is described by Cianciulli and colleagues (Figure 5) ${ }^{165}$. 


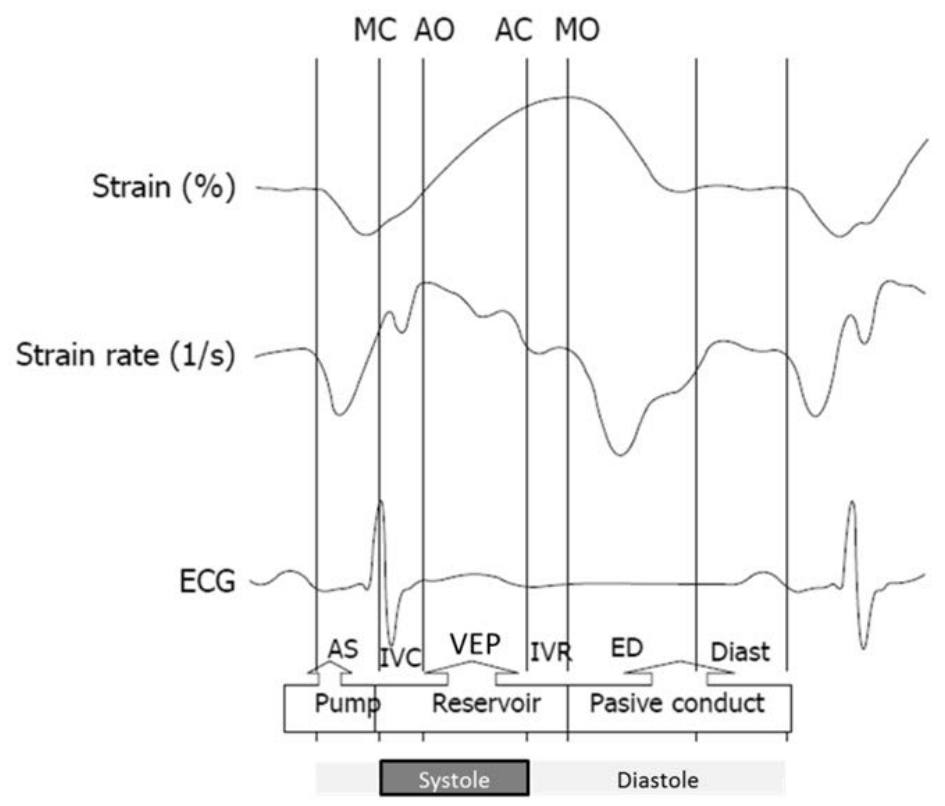

Figure 5 (after Cianciulli, 2010). Left atrial strain and strain rate during the phases of the cardiac cycle, in relation to the atrial phases: the reservoir phase, the conduit phase (passive conduct) and the active contractile phase (pump). MC: Mitral closure; AO: Aortic opening; AC: Aortic closure; MO: Mitral opening; AS: Atrial systole (= atrial contraction); IVC: Isovolumetric contraction; VEP: Ventricular Ejection Phase; IVR: Isovolumetric relaxation; ED: Early diastole; Diast: Diastasis.

\section{The papillary muscles}

Local geometrical changes, by displacement of the papillary muscles, were extensively described in the section, evaluating the left ventricular geometry (previous section). Kisanuki et al. examined the function of the papillary muscles by 2D echocardiographic fractional shortening ${ }^{72}$. The length of the ALPM and PMPM was measured at an apical 4-chamber (or apical long-axis) view and a 2-chamber view, respectively, during the end of systole and end of diastole. Fractional shortening of both PMs was decreased in patients with severe ischemic MR. A more functional measurement, regarding also the dyssynchrony of the papillary muscles has been described by Tigen et $\mathrm{al}^{166}$. These authors projected the technique of LV strain measurement by 2D speckle-tracking echocardiography on both papillary muscles. The longitudinal peak strain of the ALPM and PMPM was quantified. Tigen et al used this technique to calculate the papillary systolic dyssynchrony (DYS-PAP) in non-ischemic MR patients. The beginning of the QRS complex was used as the reference point and the time to peak (TTP) systolic longitudinal strain was quantified for each papillary muscle. For the assessment of DYS-PAP, the absolute difference in TTP between the anterolateral and posteromedial papillary 
muscle was calculated. This method of assessing the papillary muscle function and dyssynchrony will be used and explained for patients with CIMR in Chapters 4, 5 and 6 of this thesis.

\section{Surgical treatment of CIMR and outcome}

In clinical practice, the treatment for patients with CIMR is coronary artery bypass grafting (CABG) combined with mitral valve surgery, as recommended by the European guidelines (Class I recommendation for patients with severe MR and EF > 30\%; Class IIA recommendation for patients with moderate $M R$ or patients with severe $M R$ and $E F<$ $30 \%)^{108}$. The question to address the mitral valve insufficiency by mitral valve replacement or mitral valve repair, remains the subject of debate and falls outside the scope of this thesis ${ }^{167,168}$. This thesis reports on the predicting factors for recurrence of MR after CABG and restrictive annuloplasty (RA). Other operative repair techniques like Alfieri's edge-to-edge correction ${ }^{169,}{ }^{170}$, LV plication of the infarction region ${ }^{104}$, Dor restoration ${ }^{171}$, the second-order chorda cut technique ${ }^{89}$, surgical relocation of the posterior PM ${ }^{172}$, posterior leaflet extension ${ }^{173}$, the papillary sling ${ }^{174}$, external devices to restore LV geometry (Coapsys Device ${ }^{175}$, Myocor, Maple Grove, Minnesota; CorCap Cardiac Support Device ${ }^{176}$, Acorn Caridovascular Inc, St.Paul, Minnesota) or transcatheter devices (Carillon ${ }^{177}$, Cardiac Dimensions InC, Kirkland, WA, USA; MitraClip ${ }^{178}$, Abbott , Abbott Park, IL, USA ) are also outside the scope of this thesis.

\section{Outcome of CABG and restrictive annuloplasty (RA)}

For the treatment of patients with CIMR restrictive annuloplasty, combined with CABG, has been successfully used for more than a decade and has been accepted as an effective treatment for immediate improvement in mitral valve function ${ }^{3}$. Despite the enthusiasm for low perioperative mortality rate and the positive effects on survival and functional symptomatic status ${ }^{4-7}$, an increasing number of studies have clearly demonstrated that RA is subject to variable rates of recurrence of $M R^{4,8-10}$, notwithstanding good initial operative results. Recurrence of MR varies because of heterogeneity of study data, regarding population characteristics, follow up (FU) time, surgical technique (under-sizing strategy and concomitant $C A B G$ ), type of ring (rigid, flexible, semiflexible) and diagnostic measurements. Moreover, pitfalls in measuring and interpreting outcome of RA in CIMR patients, like the presence of residual MR or the bias due to combining different eras of medical practice (e.g. before and after the introduction of flexible bands or medical evidence of downsizing the annulus by two sizes), should be excluded $^{179}$.

Gelsomino et al. demonstrated that the initial results of CABG and RA were promising: at one-year FU there was no increase of MR, a significantly decrease of LV di- 
mensions and improvement of sphericity indexes ${ }^{4}$. However, at 3- and 5-year FU, a significant deterioration of these parameters was observed, resulting in more than $35 \%$ and $72 \%$ recurrence of $M R(\geq 2+)$ at 3 and 5 years, respectively. Regarding severe MR ( $\geq 3+)$, the incidence was $5 \%$ and $44 \%$ at 3 and 5 years FU, respectively. At 5-year FU, LV reverse remodeling was found in $44.2 \%$ of the study population. Crabtree et al. showed at a median FU of 1.7 years, a recurrence of $M R(\geq 3+)$ in $28 \%$ of their patients ${ }^{8}$. The adverse outcome was related to the following mechanisms: functional mitral stenosis after $\mathrm{RA}^{180}$ or the recurrence of $M R^{181}$ by continued and progressive adverse LV remodeling ${ }^{11,12,164,182}$ ensuing worsening of leaflet tethering ${ }^{180,181}$. Myocardial viability and LV size are important variables related to ventricular remodeling after revascularization and mitral annuloplasty ${ }^{183}$ although, the presence of viable myocardial segments in ischemic patients is not a guarantee for functional recovery after revascularization ${ }^{111}$.

However, recent studies have confirmed that some patients with CIMR may benefit from $R^{6,7,184}$. Surgical success was not only described by absence of recurrence of $M R$, but also by the clinical improvement (NYHA class), improved LV function (ejection fraction) and reverse remodeling of the left ventricle (LV dimensions) and left atrium (LA dimensions).

The conflicting results of RA are remarkable: Some patients clearly benefit from this procedure and others do not. Insights into the mechanism of recurrent MR as well as predictors are explained in the following paragraphs. After discussing the current findings in literature, this thesis will further elaborate on the echocardiographic predictors of recurrent MR.

\section{Mechanisms of recurrent MR}

The mechanisms, contributing to the recurrence of MR are the continuous local and global adverse LV remodeling, leading to the recurrence of mitral leaflet tethering and the hindrance of systolic mitral leaflet coaptation. Gelsomino et al. demonstrated that the lack of $L V$ reverse remodeling (LVRR) after RA led to a significant recurrence of $M R^{182}$. They defined the LVRR as a reduction of the LV ESV $>15 \%$ compared to the baseline measurement. The patients in their study had no/trivial MR at discharge, which means that the lack of LVRR was not sufficient to explain the recurrence of MR. Indeed, in this study, LV dimensions decreased and sphericity indexes improved in the group that showed postoperative LVRR, while in the group without LVRR these parameters initially improved and deteriorated at late FU. Ejection fraction and MPI improved in the group with LVRR, but remained unchanged in the group without LVRR. The mechanism of recurrent MR by continued global LV remodeling was confirmed by Hung et al. who showed increases in LV volumes and sphericity, parallel to the increase of MR severity (vena contracta and jet area/LA area), but without changes in LV function $^{11}$. However, several authors demonstrated that the local remodeling of the $L V$ 
segments supporting the papillary muscle is an essential condition for the development of recurrent $\mathrm{MR}^{12,126,130,181}$ Interestingly, in the study of Magne et al. not all the patients with recurrent MR demonstrated global LVR, defined by significant changes in LV volume and shape ${ }^{12}$. In these patients the postoperative alteration of MV configuration predominantly affected the AML, more specifically the bending of the AML to a concave configuration. They suggested that localized LV remodeling, localized impaired myocardial contractility or progressive fibrosis and stiffening of the myocardium adjacent to the PMs, was responsible for AML geometrical and functional alteration leading to MR. The contribution of the AML to postoperative MR can be reasonably explained since the restrictive annuloplasty ring transforms the MV into a mono-leaflet system, in which only the AML is mobile and closes against the relatively fixed PML. As a consequence of the alteration of the AML in a concave configuration, Magne and colleagues suggested that "chordal cutting", described by Messas et al. ${ }^{89}$ could be helpful at the time of restrictive annuloplasty to prevent this phenomenon ${ }^{12}$.

\section{Predictors of recurrent MR}

Evaluating the preoperative echocardiographic data in patients with CIMR undergoing CABG and RA, predictors of outcome can be identified. The positive predictors suggest that patients will benefit from RA, while the negative predictors denote the recurrence of MR.

Positive predictors: The surgical success of CABG and RA in CIMR is described as the absence of MR because of postoperative LV reverse remodeling (LVRR) ${ }^{7,131,184}$. This means that predictors of postoperative LVRR denote the patients who will benefit from RA. Braun and colleagues demonstrated that LV dimensions predicted the LVRR: a preoperative $L V$ end diastolic diameter $(L V E D D) \leq 65 \mathrm{~mm}$ provided a cure for patients with CIMR undergoing MV repair, while LVEDD > $65 \mathrm{~mm}$ predicted a poor outcome ${ }^{131}$. Gelsomino et al. showed that functional and geometrical preoperative parameters prognosticated the LVRR (defined as a reduction of LV ESV $>15 \%$ ) $^{182}$. The authors concluded that at multivariable analysis the baseline myocardial performance index (with a cut off: $M P I<0.9$ ), the wall motion score index (with a cut off: WMSI < 1.59) and the systolic sphericity index (with a cut off: $S I_{S}<0.72$ ) were independent predictors of LVRR. In another study, based on diastolic LV function measurements, the same group of authors defined by logistic regression analysis in their study population, that $D T \leq$ 125 ms was a strong predictor of LVRR after RA.

Negative predictors: Failure of surgical treatment in patients with CIMR is defined as a recurrence of $M R$ by progressive postoperative adverse LV remodeling. These patients do not benefit from CABG and RA. Suggestions for other surgical repair techniques, like "chorda cutting" ${ }^{90}$, Dor restoration ${ }^{171}$, surgical relocation of PMPM $^{172}$ or other techniques as well as for MV replacement ${ }^{167,168}$ are outside the scope of this thesis. 
Agricola and colleagues distinguished the patients with ischemic MR in two subgroups, based on the restricted mitral leaflet motion and leaflet tethering pattern and found a different degree of local and global LV remodeling, and characteristics of the regurgitant jet between these two populations ${ }^{129}$.

Posterior mitral leaflet tethering. Zhu et al. observed that MR was correlated with tethering of both leaflets. The coaptation length $(\mathrm{CL})$ was the primary independent determinant of preoperative and postoperative $M R^{181}$. The $A M L$ and $P M L$ tethering were the primary determinants of the preoperative and postoperative $\mathrm{CL}$, respectively, since patients with recurrent $M R$ showed no improvement of $A M L$ tethering, but a greater worsening of the PML tethering. It is clear that tethering of the posterior leaflet may be exacerbated by RA: while reducing the septal-lateral diameter, the posterior (muscular) annulus is shifted anteriorly towards the fibrous anterior annulus (fibrous skeleton of the heart). The PMPM will be relatively shifted laterally compared to the posterior annulus, causing more tethering on the PML. Kuwahara et al. ${ }^{126}$, Ciarka et al. $^{128}$ and Magne and colleagues ${ }^{127}$ confirmed that tethering of the PML was a predictor of recurrence of $M R$. The latter group demonstrated that the preoperative presence of PML angle $\left(\beta \geq 45^{\circ}\right)$ had the best performance for the prediction of persistent MR.

Anterior mitral leaflet tethering. Gelsomino and colleagues evaluated the preoperative and postoperative valvular geometrical alterations in two groups of patients (and controls): patients with and without postoperative $\mathrm{MR}^{130}$. They demonstrated that the preoperative $A M L$ angle (with a cut off: $\alpha \geq 39.5^{\circ}$ ) and the AML excursion angle (with a cut off: $\alpha E X \leq 35^{\circ}$ ) were independent predictors of recurrent $M R$, while the preoperative PML was not significantly different between both groups. Moreover, the AML/PML tethering, reflecting the tethering pattern, also predicted MR when the ratio $\alpha / \beta \geq$ 0.76. The importance of AML tethering in a large multicenter patient study, is shown in Chapter 2. We demonstrated that AML tethering was not secondary to LV function or geometry in predicting recurrent MR. We concluded that the AML tethering was in independent and primary predictor of MR recurrence. The AML tethering in relation to $M R$ and lack of LVRR is discussed in Chapter 3. The symmetric tethering pattern reflecting advanced LV mechanical dyssynchrony in patients with CIMR is discussed in Chapter 6 and the segmental strain measurement in relation to the tethering pattern is demonstrated in Chapter 7.

Coaptation height. Calafiori et al. demonstrated that coaptation height $(\mathrm{CH} \geq 11 \mathrm{~mm})$ was a predictor of recurrence of MR after CABG and RA. As a consequence, these authors changed their surgical strategy by performing $\mathrm{MV}$ replacement when the cut off value of $\mathrm{CH}$ was reached ${ }^{167}$. This predicting parameter of recurrent MR was confirmed by several authors ${ }^{127,130}$. 


\section{Conclusion and outline of this thesis}

The high rate of recurrence of ischemic MR after MV repair and CABG supports the role of left ventricular remodeling, which is a progressive phenomenon. This further underscores that ischemic MR is a disease of the ventricle, not of the valve itself. Although some patients are cured by CABG and RA, others have poor outcome due to progressive LVR. In this thesis predictive parameters will be investigated at the level of leaflet geometry and papillary muscle dyssynchrony. Segmental LV strain measurements will be related to valve geometry and left atrial strain and strain rate will be evaluated in CIMR patients. 


\section{References}

1. Grigioni F, Enriquez-Sarano M, Zehr KJ, Bailey KR, Tajik AJ. Ischemic mitral regurgitation: Long-term outcome and prognostic implications with quantitative doppler assessment. Circulation. 2001;103:1759-1764

2. Yiu SF, Enriquez-Sarano M, Tribouilloy C, Seward JB, Tajik AJ. Determinants of the degree of functional mitral regurgitation in patients with systolic left ventricular dysfunction: A quantitative clinical study. Circulation. 2000;102:1400-1406

3. Bolling SF, Pagani FD, Deeb GM, Bach DS. Intermediate-term outcome of mitral reconstruction in cardiomyopathy. The Journal of thoracic and cardiovascular surgery. 1998;115:381-386; discussion 387388

4. Gelsomino S, Lorusso R, De Cicco G, Capecchi I, Rostagno C, Caciolli S, Romagnoli S, Da Broi U, Stefano $P$, Gensini GF. Five-year echocardiographic results of combined undersized mitral ring annuloplasty and coronary artery bypass grafting for chronic ischaemic mitral regurgitation. European heart journal. 2008;29:231-240

5. Bax JJ, Braun J, Somer ST, Klautz R, Holman ER, Versteegh MI, Boersma E, Schalij MJ, van der Wall EE, Dion RA. Restrictive annuloplasty and coronary revascularization in ischemic mitral regurgitation results in reverse left ventricular remodeling. Circulation. 2004;110:II103-II108

6. Braun J, van de Veire NR, Klautz RJ, Versteegh MI, Holman ER, Westenberg JJ, Boersma E, van der Wall $\mathrm{EE}, \mathrm{Bax} \mathrm{JJ}$, Dion RA. Restrictive mitral annuloplasty cures ischemic mitral regurgitation and heart failure. The Annals of thoracic surgery. 2008;85:430-436; discussion 436-437

7. Geidel S, Lass M, Schneider C, Groth G, Boczor S, Kuck KH, Ostermeyer J. Downsizing of the mitral valve and coronary revascularization in severe ischemic mitral regurgitation results in reverse left ventricular and left atrial remodeling. European journal of cardio-thoracic surgery: official journal of the European Association for Cardio-thoracic Surgery. 2005;27:1011-1016

8. Crabtree TD, Bailey MS, Moon MR, Munfakh N, Pasque MK, Lawton JS, Moazami N, Aubuchon KA, AlDadah AS, Damiano RJ, Jr. Recurrent mitral regurgitation and risk factors for early and late mortality after mitral valve repair for functional ischemic mitral regurgitation. The Annals of thoracic surgery. 2008;85:1537-1542; discussion 1542-1543

9. Mihaljevic T, Lam BK, Rajeswaran J, Takagaki M, Lauer MS, Gillinov AM, Blackstone EH, Lytle BW. Impact of mitral valve annuloplasty combined with revascularization in patients with functional ischemic mitral regurgitation. Journal of the American College of Cardiology. 2007;49:2191-2201

10. Serri K, Bouchard D, Demers P, Coutu M, Pellerin M, Carrier M, Perrault LP, Cartier R, Page P, Cossette $\mathrm{M}$, Basmadjian AJ. Is a good perioperative echocardiographic result predictive of durability in ischemic mitral valve repair? The Journal of thoracic and cardiovascular surgery. 2006;131:565-573 e562

11. Hung J, Papakostas L, Tahta SA, Hardy BG, Bollen BA, Duran CM, Levine RA. Mechanism of recurrent ischemic mitral regurgitation after annuloplasty: Continued Iv remodeling as a moving target. Circulation. 2004;110:II85-II90

12. Magne J, Pibarot P, Dumesnil JG, Senechal M. Continued global left ventricular remodeling is not the sole mechanism responsible for the late recurrence of ischemic mitral regurgitation after restrictive annuloplasty. Journal of the American Society of Echocardiography: official publication of the American Society of Echocardiography. 2009;22:1256-1264

13. Carpentier A. Cardiac valve surgery--the "french correction". The Journal of thoracic and cardiovascular surgery. 1983;86:323-337

14. Messas E, Pouzet B, Touchot B, Guerrero JL, Vlahakes GJ, Desnos M, Menasche P, Hagege A, Levine RA. Efficacy of chordal cutting to relieve chronic persistent ischemic mitral regurgitation. Circulation. 2003;108 Suppl 1:II111-II115

15. Kumar AS, Talwar S, Saxena A, Singh R, Velayoudam D. Results of mitral valve repair in rheumatic mitral regurgitation. Interactive cardiovascular and thoracic surgery. 2006;5:356-361 
16. Adams DH, Anyanwu AC, Rahmanian PB, Abascal V, Salzberg SP, Filsoufi F. Large annuloplasty rings facilitate mitral valve repair in barlow's disease. The Annals of thoracic surgery. 2006;82:2096-2100; discussion 2101

17. Anyanwu AC, Adams DH. Etiologic classification of degenerative mitral valve disease: Barlow's disease and fibroelastic deficiency. Seminars in thoracic and cardiovascular surgery. 2007;19:90-96

18. Carpentier A, Chauvaud S, Fabiani JN, Deloche A, Relland J, Lessana A, D'Allaines C, Blondeau P, Piwnica A, Dubost C. Reconstructive surgery of mitral valve incompetence: Ten-year appraisal. The Journal of thoracic and cardiovascular surgery. 1980;79:338-348

19. Pasquier M, Sierro C, Yersin B, Delay D, Carron PN. Traumatic mitral valve injury after blunt chest trauma: A case report and review of the literature. The Journal of trauma. 2010;68:243-246

20. Iung B, Rousseau-Paziaud J, Cormier B, Garbarz E, Fondard O, Brochet E, Acar C, Couetil JP, Hvass U, Vahanian A. Contemporary results of mitral valve repair for infective endocarditis. Journal of the American College of Cardiology. 2004;43:386-392

21. Fatkin D, MacRae C, Sasaki T, Wolff MR, Porcu M, Frenneaux M, Atherton J, Vidaillet HJ, Jr., Spudich S, De Girolami U, Seidman JG, Seidman C, Muntoni F, Muehle G, Johnson W, McDonough B. Missense mutations in the rod domain of the lamin a/c gene as causes of dilated cardiomyopathy and conduction-system disease. The New England journal of medicine. 1999;341:1715-1724

22. Kuhl U, Pauschinger M, Noutsias M, Seeberg B, Bock T, Lassner D, Poller W, Kandolf R, Schultheiss HP. High prevalence of viral genomes and multiple viral infections in the myocardium of adults with "idiopathic" left ventricular dysfunction. Circulation. 2005;111:887-893

23. Hequet O, Le QH, Moullet I, Pauli E, Salles G, Espinouse D, Dumontet C, Thieblemont C, Arnaud P, Antal D, Bouafia F, Coiffier B. Subclinical late cardiomyopathy after doxorubicin therapy for lymphoma in adults. Journal of clinical oncology: official journal of the American Society of Clinical Oncology. 2004;22:1864-1871

24. Kalaria VG, Passannante MR, Shah T, Modi K, Weisse AB. Effect of mitral regurgitation on left ventricular thrombus formation in dilated cardiomyopathy. American heart journal. 1998;135:215-220

25. Om A, Ellahham S, Ornato JP. Reversibility of cocaine-induced cardiomyopathy. American heart journal. 1992;124:1639-1641

26. Birnbaum Y, Chamoun AJ, Conti VR, Uretsky BF. Mitral regurgitation following acute myocardial infarction. Coronary artery disease. 2002;13:337-344

27. Kishon Y, Oh JK, Schaff HV, Mullany CJ, Tajik AJ, Gersh BJ. Mitral valve operation in postinfarction rupture of a papillary muscle: Immediate results and long-term follow-up of 22 patients. Mayo Clinic proceedings. Mayo Clinic. 1992;67:1023-1030

28. Braun J, Voigt PG, Versteegh MI, Dion RA. Restrictive mitral annuloplasty in refractory cardiogenic shock with acute postinfarction mitral insufficiency and intact papillary muscle. The Journal of thoracic and cardiovascular surgery. 2003;126:284-286

29. Hickey MS, Smith LR, Muhlbaier LH, Harrell FE, Jr., Reves JG, Hinohara T, Califf RM, Pryor DB, Rankin JS. Current prognosis of ischemic mitral regurgitation. Implications for future management. Circulation. 1988;78:I51-I59

30. Lamas GA, Mitchell GF, Flaker GC, Smith SC, Jr., Gersh BJ, Basta L, Moye L, Braunwald E, Pfeffer MA. Clinical significance of mitral regurgitation after acute myocardial infarction. Survival and ventricular enlargement investigators. Circulation. 1997;96:827-833

31. Tcheng JE, Jackman JD, Jr., Nelson CL, Gardner LH, Smith LR, Rankin JS, Califf RM, Stack RS. Outcome of patients sustaining acute ischemic mitral regurgitation during myocardial infarction. Annals of internal medicine. 1992;117:18-24

32. Aronson D, Goldsher N, Zukermann R, Kapeliovich M, Lessick J, Mutlak D, Dabbah S, Markiewicz W, Beyar R, Hammerman $\mathrm{H}$, Reisner S, Agmon Y. Ischemic mitral regurgitation and risk of heart failure after myocardial infarction. Archives of internal medicine. 2006;166:2362-2368

33. Bursi F, Enriquez-Sarano M, Nkomo VT, Jacobsen SJ, Weston SA, Meverden RA, Roger VL. Heart failure and death after myocardial infarction in the community: The emerging role of mitral regurgitation. Circulation. 2005;111:295-301 
34. Roger VL, Go AS, Lloyd-Jones DM, Adams RJ, Berry JD, Brown TM, Carnethon MR, Dai S, de Simone G, Ford ES, Fox CS, Fullerton HJ, Gillespie C, Greenlund KJ, Hailpern SM, Heit JA, Ho PM, Howard VJ, Kissela BM, Kittner SJ, Lackland DT, Lichtman JH, Lisabeth LD, Makuc DM, Marcus GM, Marelli A, Matchar DB, McDermott MM, Meigs JB, Moy CS, Mozaffarian D, Mussolino ME, Nichol G, Paynter NP, Rosamond WD, Sorlie PD, Stafford RS, Turan TN, Turner MB, Wong ND, Wylie-Rosett J. Heart disease and stroke statistics--2011 update: A report from the american heart association. Circulation. 2011;123:e18-e209

35. Felker GM, Shaw LK, O'Connor CM. A standardized definition of ischemic cardiomyopathy for use in clinical research. Journal of the American College of Cardiology. 2002;39:210-218

36. Adams KF, Jr., Dunlap SH, Sueta CA, Clarke SW, Patterson JH, Blauwet MB, Jensen LR, Tomasko L, Koch G. Relation between gender, etiology and survival in patients with symptomatic heart failure. Journal of the American College of Cardiology. 1996;28:1781-1788

37. Bart BA, Shaw LK, McCants CB, Jr., Fortin DF, Lee KL, Califf RM, O'Connor CM. Clinical determinants of mortality in patients with angiographically diagnosed ischemic or nonischemic cardiomyopathy. Journal of the American College of Cardiology. 1997;30:1002-1008

38. Gheorghiade M, Bonow RO. Chronic heart failure in the united states: A manifestation of coronary artery disease. Circulation. 1998;97:282-289

39. Patel JB, Borgeson DD, Barnes ME, Rihal CS, Daly RC, Redfield MM. Mitral regurgitation in patients with advanced systolic heart failure. Journal of cardiac failure. 2004;10:285-291

40. Rossi A, Dini FL, Faggiano P, Agricola E, Cicoira M, Frattini S, Simioniuc A, Gullace M, Ghio S, EnriquezSarano M, Temporelli PL. Independent prognostic value of functional mitral regurgitation in patients with heart failure. A quantitative analysis of 1256 patients with ischaemic and non-ischaemic dilated cardiomyopathy. Heart. 2011;97:1675-1680

41. Di Mauro M, Di Giammarco G, Vitolla G, Contini M, laco AL, Bivona A, Weltert L, Calafiore AM. Impact of no-to-moderate mitral regurgitation on late results after isolated coronary artery bypass grafting in patients with ischemic cardiomyopathy. The Annals of thoracic surgery. 2006;81:2128-2134

42. Kang DH, Sun BJ, Kim DH, Yun SC, Song JM, Choo SJ, Chung CH, Song JK, Lee JW, Park SW, Park SJ. Percutaneous versus surgical revascularization in patients with ischemic mitral regurgitation. Circulation. 2011;124:S156-S162

43. Kanzaki H, Bazaz R, Schwartzman D, Dohi K, Sade LE, Gorcsan J, 3rd. A mechanism for immediate reduction in mitral regurgitation after cardiac resynchronization therapy: Insights from mechanical activation strain mapping. Journal of the American College of Cardiology. 2004;44:1619-1625

44. Perloff JK, Roberts WC. The mitral apparatus. Functional anatomy of mitral regurgitation. Circulation. 1972;46:227-239

45. Itoh A, Ennis DB, Bothe W, Swanson JC, Krishnamurthy G, Nguyen TC, Ingels NB, Jr., Miller DC. Mitral annular hinge motion contribution to changes in mitral septal-lateral dimension and annular area. The Journal of thoracic and cardiovascular surgery. 2009;138:1090-1099

46. Timek TA, Green GR, Tibayan FA, Lai DT, Rodriguez F, Liang D, Daughters GT, Ingels NB, Jr., Miller DC. Aorto-mitral annular dynamics. The Annals of thoracic surgery. 2003;76:1944-1950

47. Tsakiris AG, Von Bernuth G, Rastelli GC, Bourgeois MJ, Titus JL, Wood EH. Size and motion of the mitral valve annulus in anesthetized intact dogs. Journal of applied physiology. 1971;30:611-618

48. Ormiston JA, Shah PM, Tei C, Wong M. Size and motion of the mitral valve annulus in man. I. A twodimensional echocardiographic method and findings in normal subjects. Circulation. 1981;64:113-120

49. Timek TA, Lai DT, Dagum P, Green GR, Glasson JR, Daughters GT, Ingels NB, Jr., Miller DC. Mitral annular dynamics during rapid atrial pacing. Surgery. 2000;128:361-367

50. Kwan J, Jeon MJ, Kim DH, Park KS, Lee WH. Does the mitral annulus shrink or enlarge during systole? A real-time 3d echocardiography study. Journal of Korean medical science. 2009;24:203-208

51. Pai RG, Tanimoto M, Jintapakorn W, Azevedo J, Pandian NG, Shah PM. Volume-rendered threedimensional dynamic anatomy of the mitral annulus using a transesophageal echocardiographic technique. The Journal of heart valve disease. 1995;4:623-627 
52. Flachskampf FA, Chandra S, Gaddipatti A, Levine RA, Weyman AE, Ameling W, Hanrath P, Thomas JD. Analysis of shape and motion of the mitral annulus in subjects with and without cardiomyopathy by echocardiographic 3-dimensional reconstruction. Journal of the American Society of Echocardiography: official publication of the American Society of Echocardiography. 2000;13:277-287

53. Glasson JR, Komeda MK, Daughters GT, Niczyporuk MA, Bolger AF, Ingels NB, Miller DC. Threedimensional regional dynamics of the normal mitral anulus during left ventricular ejection. The Journal of thoracic and cardiovascular surgery. 1996;111:574-585

54. Komoda T, Hetzer R, Uyama C, Siniawski H, Maeta H, Rosendahl UP, Ozaki K. Mitral annular function assessed by 3d imaging for mitral valve surgery. The Journal of heart valve disease. 1994;3:483-490

55. Levine RA, Handschumacher MD, Sanfilippo AJ, Hagege AA, Harrigan P, Marshall JE, Weyman AE. Threedimensional echocardiographic reconstruction of the mitral valve, with implications for the diagnosis of mitral valve prolapse. Circulation. 1989;80:589-598

56. Victor S, Nayak VM. Variations in the papillary muscles of the normal mitral valve and their surgical relevance. Journal of cardiac surgery. 1995;10:597-607

57. Ritchie J, Warnock JN, Yoganathan AP. Structural characterization of the chordae tendineae in native porcine mitral valves. The Annals of thoracic surgery. 2005;80:189-197

58. Ranganathan N, Burch GE. Gross morphology and arterial supply of the papillary muscles of the left ventricle of man. American heart journal. 1969;77:506-516

59. Voci P, Bilotta F, Caretta Q, Mercanti C, Marino B. Papillary muscle perfusion pattern. A hypothesis for ischemic papillary muscle dysfunction. Circulation. 1995;91:1714-1718

60. Kumanohoso T, Otsuji Y, Yoshifuku S, Matsukida K, Koriyama C, Kisanuki A, Minagoe S, Levine RA, Tei C. Mechanism of higher incidence of ischemic mitral regurgitation in patients with inferior myocardial infarction: Quantitative analysis of left ventricular and mitral valve geometry in 103 patients with prior myocardial infarction. The Journal of thoracic and cardiovascular surgery. 2003;125:135-143

61. Joudinaud TM, Kegel CL, Flecher EM, Weber PA, Lansac E, Hvass U, Duran CM. The papillary muscles as shock absorbers of the mitral valve complex. An experimental study. European journal of cardiothoracic surgery: official journal of the European Association for Cardio-thoracic Surgery. 2007;32:96101

62. Simonson JS, Schiller NB. Descent of the base of the left ventricle: An echocardiographic index of left ventricular function. Journal of the American Society of Echocardiography: official publication of the American Society of Echocardiography. 1989;2:25-35

63. Rumberger JA. Ventricular dilatation and remodeling after myocardial infarction. Mayo Clinic proceedings. Mayo Clinic. 1994;69:664-674

64. Levine RA, Hung J, Otsuji Y, Messas E, Liel-Cohen N, Nathan N, Handschumacher MD, Guerrero JL, He S, Yoganathan AP, Vlahakes GJ. Mechanistic insights into functional mitral regurgitation. Current cardiology reports. 2002;4:125-129

65. Burch GE, De Pasquale NP, Phillips JH. Clinical manifestations of papillary muscle dysfunction. Archives of internal medicine. 1963;112:112-117

66. Miller GE, Jr., Kerth WJ, Gerbode F. Experimental papillary muscle infarction. The Journal of thoracic and cardiovascular surgery. 1968;56:611-616

67. Tsakiris AG, Rastelli GC, Amorim Dde S, Titus JL, Wood EH. Effect of experimental papillary muscle damage on mitral valve closure in intact anesthetized dogs. Mayo Clinic proceedings. Mayo Clinic. 1970;45:275-285

68. Mittal AK, Langston M, Jr., Cohn KE, Selzer A, Kerth WJ. Combined papillary muscle and left ventricular wall dysfunction as a cause of mitral regurgitation. An experimental study. Circulation. 1971;44:174180

69. Burch GE, DePasquale NP, Phillips JH. The syndrome of papillary muscle dysfunction. American heart journal. 1968;75:399-415

70. Jouan J, Tapia M, R CC, Lansac E, Acar C. Ischemic mitral valve prolapse: Mechanisms and implications for valve repair. European journal of cardio-thoracic surgery: official journal of the European Association for Cardio-thoracic Surgery. 2004;26:1112-1117 
71. Godley RW, Wann LS, Rogers EW, Feigenbaum H, Weyman AE. Incomplete mitral leaflet closure in patients with papillary muscle dysfunction. Circulation. 1981;63:565-571

72. Kisanuki A, Otsuji Y, Kuroiwa R, Murayama T, Matsushita R, Shibata K, Yutsudo T, Nakao S, Nomoto K, Tomari $\mathrm{T}$, et al. Two-dimensional echocardiographic assessment of papillary muscle contractility in patients with prior myocardial infarction. Journal of the American College of Cardiology. 1993;21:932938

73. Kaul S, Spotnitz WD, Glasheen WP, Touchstone DA. Mechanism of ischemic mitral regurgitation. An experimental evaluation. Circulation. 1991;84:2167-2180

74. Dent JM, Spotnitz WD, Nolan SP, Jayaweera AR, Glasheen WP, Kaul S. Mechanism of mitral leaflet excursion. The American journal of physiology. 1995;269:H2100-H2108

75. Otsuji Y, Handschumacher MD, Schwammenthal E, Jiang L, Song JK, Guerrero JL, Vlahakes GJ, Levine RA. Insights from three-dimensional echocardiography into the mechanism of functional mitral regurgitation: Direct in vivo demonstration of altered leaflet tethering geometry. Circulation. 1997;96:1999-2008

76. Messas E, Guerrero JL, Handschumacher MD, Chow CM, Sullivan S, Schwammenthal E, Levine RA. Paradoxic decrease in ischemic mitral regurgitation with papillary muscle dysfunction: Insights from three-dimensional and contrast echocardiography with strain rate measurement. Circulation. 2001;104:1952-1957

77. Khankirawatana B, Khankirawatana S, Mahrous H, Porter TR. Assessment of papillary muscle function using myocardial velocity gradient derived from tissue doppler echocardiography. The American journal of cardiology. 2004;94:45-49

78. He S, Fontaine AA, Schwammenthal E, Yoganathan AP, Levine RA. Integrated mechanism for functional mitral regurgitation: Leaflet restriction versus coapting force: In vitro studies. Circulation. 1997;96:1826-1834

79. Kono T, Sabbah HN, Rosman H, Alam M, Jafri S, Goldstein S. Left ventricular shape is the primary determinant of functional mitral regurgitation in heart failure. Journal of the American College of Cardiology. 1992;20:1594-1598

80. Sabbah HN, Kono T, Stein PD, Mancini GB, Goldstein S. Left ventricular shape changes during the course of evolving heart failure. The American journal of physiology. 1992;263: $\mathrm{H} 266-\mathrm{H} 270$

81. Sabbah HN, Rosman H, Kono T, Alam M, Khaja F, Goldstein S. On the mechanism of functional mitral regurgitation. The American journal of cardiology. 1993;72:1074-1076

82. Abraham WT, Fisher WG, Smith AL, Delurgio DB, Leon AR, Loh E, Kocovic DZ, Packer M, Clavell AL, Hayes DL, Ellestad M, Trupp RJ, Underwood J, Pickering F, Truex C, McAtee P, Messenger J. Cardiac resynchronization in chronic heart failure. The New England journal of medicine. 2002;346:1845-1853

83. Yu CM, Chau E, Sanderson JE, Fan K, Tang MO, Fung WH, Lin H, Kong SL, Lam YM, Hill MR, Lau CP. Tissue doppler echocardiographic evidence of reverse remodeling and improved synchronicity by simultaneously delaying regional contraction after biventricular pacing therapy in heart failure. Circulation. 2002;105:438-445

84. Ahmad RM, Gillinov AM, McCarthy PM, Blackstone EH, Apperson-Hansen C, Qin JX, Agler D, Shiota T, Cosgrove DM. Annular geometry and motion in human ischemic mitral regurgitation: Novel assessment with three-dimensional echocardiography and computer reconstruction. The Annals of thoracic surgery. 2004;78:2063-2068; discussion 2068

85. Tibayan FA, Rodriguez F, Langer F, Zasio MK, Bailey L, Liang D, Daughters GT, Ingels NB, Jr., Miller DC. Annular remodeling in chronic ischemic mitral regurgitation: Ring selection implications. The Annals of thoracic surgery. 2003;76:1549-1554; discussion 1554-1545

86. Otsuji Y, Kumanohoso T, Yoshifuku S, Matsukida K, Koriyama C, Kisanuki A, Minagoe S, Levine RA, Tei C. Isolated annular dilation does not usually cause important functional mitral regurgitation: Comparison between patients with lone atrial fibrillation and those with idiopathic or ischemic cardiomyopathy. Journal of the American College of Cardiology. 2002;39:1651-1656 
87. Watanabe N, Ogasawara Y, Yamaura Y, Kawamoto T, Akasaka T, Yoshida K. Geometric deformity of the mitral annulus in patients with ischemic mitral regurgitation: A real-time three-dimensional echocardiographic study. The Journal of heart valve disease. 2005;14:447-452

88. Alkadhi H, Desbiolles L, Stolzmann P, Leschka S, Scheffel H, Plass A, Schertler T, Trindade PT, Genoni M, Cattin P, Marincek B, Frauenfelder T. Mitral annular shape, size, and motion in normals and in patients with cardiomyopathy: Evaluation with computed tomography. Investigative radiology. 2009;44:218225

89. Messas E, Guerrero JL, Handschumacher MD, Conrad C, Chow CM, Sullivan S, Yoganathan AP, Levine RA. Chordal cutting: A new therapeutic approach for ischemic mitral regurgitation. Circulation. 2001;104:1958-1963

90. Messas E, Bel A, Szymanski C, Cohen I, Touchot B, Handschumacher MD, Desnos M, Carpentier A, Menasche $P$, Hagege AA, Levine RA. Relief of mitral leaflet tethering following chronic myocardial infarction by chordal cutting diminishes left ventricular remodeling. Circulation. Cardiovascular imaging. 2010;3:679-686

91. Rodriguez F, Langer F, Harrington KB, Tibayan FA, Zasio MK, Liang D, Daughters GT, Ingels NB, Miller DC. Cutting second-order chords does not prevent acute ischemic mitral regurgitation. Circulation. 2004;110:II91-II97

92. Rodriguez F, Langer F, Harrington KB, Tibayan FA, Zasio MK, Cheng A, Liang D, Daughters GT, Covell JW, Criscione JC, Ingels NB, Miller DC. Importance of mitral valve second-order chordae for left ventricular geometry, wall thickening mechanics, and global systolic function. Circulation. 2004;110:II115-II122

93. van Rijk-Zwikker GL, Delemarre BJ, Huysmans HA. Mitral valve anatomy and morphology: Relevance to mitral valve replacement and valve reconstruction. Journal of cardiac surgery. 1994;9:255-261

94. Lomholt M, Nielsen SL, Hansen SB, Andersen NT, Hasenkam JM. Differential tension between secondary and primary mitral chordae in an acute in-vivo porcine model. The Journal of heart valve disease. 2002;11:337-345

95. Tanimoto $T$, Imanishi T, Kitabata H, Nakamura N, Kimura K, Yamano T, Ishibashi K, Komukai K, Ino $\mathrm{Y}$, Takarada S, Kubo T, Hirata K, Mizukoshi M, Tanaka A, Akasaka T. Prevalence and clinical significance of papillary muscle infarction detected by late gadolinium-enhanced magnetic resonance imaging in patients with st-segment elevation myocardial infarction. Circulation. 2010;122:2281-2287

96. Uemura T, Otsuji Y, Nakashiki K, Yoshifuku S, Maki Y, Yu B, Mizukami N, Kuwahara E, Hamasaki S, Biro S, Kisanuki A, Minagoe S, Levine RA, Tei C. Papillary muscle dysfunction attenuates ischemic mitral regurgitation in patients with localized basal inferior left ventricular remodeling: Insights from tissue doppler strain imaging. Journal of the American College of Cardiology. 2005;46:113-119

97. Breithardt OA, Sinha AM, Schwammenthal E, Bidaoui N, Markus KU, Franke A, Stellbrink C. Acute effects of cardiac resynchronization therapy on functional mitral regurgitation in advanced systolic heart failure. Journal of the American College of Cardiology. 2003;41:765-770

98. Lancellotti P, Melon P, Sakalihasan N, Waleffe A, Dubois C, Bertholet M, Pierard LA. Effect of cardiac resynchronization therapy on functional mitral regurgitation in heart failure. The American journal of cardiology. 2004;94:1462-1465

99. Agricola E, Oppizzi M, Galderisi M, Pisani M, Meris A, Pappone C, Margonato A. Role of regional mechanical dyssynchrony as a determinant of functional mitral regurgitation in patients with left ventricular systolic dysfunction. Heart. 2006;92:1390-1395

100. Van Garsse L, Gelsomino S, Luca F, Parise O, Lorusso R, Cheriex E, Caciolli S, Vizzardi E, Rao CM, Carella $\mathrm{R}$, Gensini GF, Maessen J. Left ventricular dyssynchrony is associated with recurrence of ischemic mitral regurgitation after restrictive annuloplasty. International journal of cardiology. 2012

101. Van Garsse L, Gelsomino S, Parise O, Luca F, Cheriex E, Lorusso R, Vizzardi E, Rao CM, Gensini GF, Maessen J. Systolic papillary muscle dyssynchrony predicts recurrence of mitral regurgitation in patients with ischemic cardiomyopathy (icm) undergoing mitral valve repair. Echocardiography. 2012 
102. Van Garsse L, Gelsomino S, Cheriex E, Luca F, Rao CM, Parise O, Gensini GF, Maessen J. Tethering symmetry reflects advanced left ventricular mechanical dyssynchrony in patients with ischemic mitral regurgitation undergoing restrictive mitral valve repair. The Annals of thoracic surgery. 2012;94:14181428

103. Kono T, Sabbah HN, Rosman H, Alam M, Jafri S, Stein PD, Goldstein S. Mechanism of functional mitral regurgitation during acute myocardial ischemia. Journal of the American College of Cardiology. 1992;19:1101-1105

104. Liel-Cohen N, Guerrero JL, Otsuji Y, Handschumacher MD, Rudski LG, Hunziker PR, Tanabe H, ScherrerCrosbie M, Sullivan S, Levine RA. Design of a new surgical approach for ventricular remodeling to relieve ischemic mitral regurgitation: Insights from 3-dimensional echocardiography. Circulation. 2000;101:2756-2763

105. Otsuji Y, Handschumacher MD, Liel-Cohen N, Tanabe H, Jiang L, Schwammenthal E, Guerrero JL, Nicholls LA, Vlahakes GJ, Levine RA. Mechanism of ischemic mitral regurgitation with segmental left ventricular dysfunction: Three-dimensional echocardiographic studies in models of acute and chronic progressive regurgitation. Journal of the American College of Cardiology. 2001;37:641-648

106. Lehmann KG, Francis CK, Dodge HT. Mitral regurgitation in early myocardial infarction. Incidence, clinical detection, and prognostic implications. Timi study group. Annals of internal medicine. 1992;117:10-17

107. Bursi F, Enriquez-Sarano M, Jacobsen SJ, Roger VL. Mitral regurgitation after myocardial infarction: A review. The American journal of medicine. 2006;119:103-112

108. Vahanian A, Alfieri O, Andreotti F, Antunes MJ, Baron-Esquivias G, Baumgartner H, Borger MA, Carrel TP, De Bonis M, Evangelista A, Falk V, lung B, Lancellotti P, Pierard L, Price S, Schafers HJ, Schuler G, Stepinska J, Swedberg K, Takkenberg J, Von Oppell UO, Windecker S, Zamorano JL, Zembala M, Bax JJ, Ceconi C, Dean V, Deaton C, Fagard R, Funck-Brentano C, Hasdai D, Hoes A, Kirchhof P, Knuuti J, Kolh P, McDonagh T, Moulin C, Popescu BA, Reiner Z, Sechtem U, Sirnes PA, Tendera M, Torbicki A, Von Segesser L, Badano LP, Bunc M, Claeys MJ, Drinkovic N, Filippatos G, Habib G, Kappetein AP, Kassab R, Lip GY, Moat N, Nickenig G, Otto CM, Pepper J, Piazza N, Pieper PG, Rosenhek R, Shuka N, Schwammenthal E, Schwitter J, Mas PT, Trindade PT, Walther T. Guidelines on the management of valvular heart disease (version 2012): The joint task force on the management of valvular heart disease of the european society of cardiology (esc) and the european association for cardio-thoracic surgery (eacts). European heart journal. 2012;33:2451-2496

109. Schuster A, Morton G, Chiribiri A, Perera D, Vanoverschelde JL, Nagel E. Imaging in the management of ischemic cardiomyopathy: Special focus on magnetic resonance. Journal of the American College of Cardiology. 2012;59:359-370

110. Kim RJ, Wu E, Rafael A, Chen EL, Parker MA, Simonetti O, Klocke FJ, Bonow RO, Judd RM. The use of contrast-enhanced magnetic resonance imaging to identify reversible myocardial dysfunction. The New England journal of medicine. 2000;343:1445-1453

111. Bax JJ, Schinkel AF, Boersma E, Elhendy A, Rizzello V, Maat A, Roelandt JR, van der Wall EE, Poldermans D. Extensive left ventricular remodeling does not allow viable myocardium to improve in left ventricular ejection fraction after revascularization and is associated with worse long-term prognosis. Circulation. 2004;110:II18-22

112. Aklog L, Filsoufi F, Flores KQ, Chen RH, Cohn LH, Nathan NS, Byrne JG, Adams DH. Does coronary artery bypass grafting alone correct moderate ischemic mitral regurgitation? Circulation. 2001;104:168-175

113. Bach DS, Deeb GM, Bolling SF. Accuracy of intraoperative transesophageal echocardiography for estimating the severity of functional mitral regurgitation. The American journal of cardiology. 1995;76:508-512

114. Grewal KS, Malkowski MJ, Piracha AR, Astbury JC, Kramer CM, Dianzumba S, Reichek N. Effect of general anesthesia on the severity of mitral regurgitation by transesophageal echocardiography. The American journal of cardiology. 2000;85:199-203 
115. Dion R, Benetis R, Elias B, Guennaoui T, Raphael D, Van Dyck M, Noirhomme P, Van Overschelde JL. Mitral valve procedures in ischemic regurgitation. The Journal of heart valve disease. 1995;4 Suppl 2:S124-129; discussion S129-131

116. Byrne JG, Aklog L, Adams DH. Assessment and management of functional or ischaemic mitral regurgitation. Lancet. 2000;355:1743-1744

117. Mihalatos DG, Gopal AS, Kates R, Toole RS, Bercow NR, Lamendola C, Berkay SH, Damus P, Robinson N, Grimson R, Shen K, Reichek N. Intraoperative assessment of mitral regurgitation: Role of phenylephrine challenge. Journal of the American Society of Echocardiography: official publication of the American Society of Echocardiography. 2006;19:1158-1164

118. Shiran A, Merdler A, Ismir E, Ammar R, Zlotnick AY, Aravot D, Lazarovici H, Zisman E, Pizov R, Lewis BS. Intraoperative transesophageal echocardiography using a quantitative dynamic loading test for the evaluation of ischemic mitral regurgitation. Journal of the American Society of Echocardiography: official publication of the American Society of Echocardiography. 2007;20:690-697

119. Spain MG, Smith MD, Grayburn PA, Harlamert EA, DeMaria AN. Quantitative assessment of mitral regurgitation by doppler color flow imaging: Angiographic and hemodynamic correlations. Journal of the American College of Cardiology. 1989;13:585-590

120. Enriquez-Sarano M, Seward JB, Bailey KR, Tajik AJ. Effective regurgitant orifice area: A noninvasive doppler development of an old hemodynamic concept. Journal of the American College of Cardiology. 1994;23:443-451

121. Zoghbi WA, Enriquez-Sarano M, Foster E, Grayburn PA, Kraft CD, Levine RA, Nihoyannopoulos $P$, Otto CM, Quinones MA, Rakowski H, Stewart WJ, Waggoner A, Weissman NJ. Recommendations for evaluation of the severity of native valvular regurgitation with two-dimensional and doppler echocardiography. Journal of the American Society of Echocardiography: official publication of the American Society of Echocardiography. 2003;16:777-802

122. Lancellotti P, Lebrun F, Pierard LA. Determinants of exercise-induced changes in mitral regurgitation in patients with coronary artery disease and left ventricular dysfunction. Journal of the American College of Cardiology. 2003;42:1921-1928

123. Lancellotti $P$, Troisfontaines $P$, Toussaint AC, Pierard LA. Prognostic importance of exercise-induced changes in mitral regurgitation in patients with chronic ischemic left ventricular dysfunction. Circulation. 2003;108:1713-1717

124. Pierard LA, Lancellotti P. The role of ischemic mitral regurgitation in the pathogenesis of acute pulmonary edema. The New England journal of medicine. 2004;351:1627-1634

125. Dion R. Invited commentary. The Annals of thoracic surgery. 2005;80:577-578

126. Kuwahara E, Otsuji Y, Iguro Y, Ueno T, Zhu F, Mizukami N, Kubota K, Nakashiki K, Yuasa T, Yu B, Uemura T, Takasaki K, Miyata M, Hamasaki S, Kisanuki A, Levine RA, Sakata R, Tei C. Mechanism of recurrent/persistent ischemic/functional mitral regurgitation in the chronic phase after surgical annuloplasty: Importance of augmented posterior leaflet tethering. Circulation. 2006;114:1529-1534

127. Magne J, Pibarot P, Dagenais F, Hachicha Z, Dumesnil JG, Senechal M. Preoperative posterior leaflet angle accurately predicts outcome after restrictive mitral valve annuloplasty for ischemic mitral regurgitation. Circulation. 2007;115:782-791

128. Ciarka A, Braun J, Delgado V, Versteegh M, Boersma E, Klautz R, Dion R, Bax JJ, Van de Veire N. Predictors of mitral regurgitation recurrence in patients with heart failure undergoing mitral valve annuloplasty. The American journal of cardiology. 2010;106:395-401

129. Agricola E, Oppizzi M, Maisano F, De Bonis M, Schinkel AF, Torracca L, Margonato A, Melisurgo G, Alfieri O. Echocardiographic classification of chronic ischemic mitral regurgitation caused by restricted motion according to tethering pattern. European journal of echocardiography: the journal of the Working Group on Echocardiography of the European Society of Cardiology. 2004;5:326-334

130. Gelsomino S, Lorusso R, Caciolli S, Capecchi I, Rostagno C, Chioccioli M, De Cicco G, Bille G, Stefano P, Gensini GF. Insights on left ventricular and valvular mechanisms of recurrent ischemic mitral regurgitation after restrictive annuloplasty and coronary artery bypass grafting. The Journal of thoracic and cardiovascular surgery. 2008;136:507-518 
131. Braun J, Bax JJ, Versteegh MI, Voigt PG, Holman ER, Klautz RJ, Boersma E, Dion RA. Preoperative left ventricular dimensions predict reverse remodeling following restrictive mitral annuloplasty in ischemic mitral regurgitation. European journal of cardio-thoracic surgery: official journal of the European Association for Cardio-thoracic Surgery. 2005;27:847-853

132. Lee LS, Kwon MH, Cevasco M, Schmitto JD, Mokashi SA, McGurk S, Cohn LH, Bolman RM, 3rd, Chen FY. Postoperative recurrence of mitral regurgitation after annuloplasty for functional mitral regurgitation. The Annals of thoracic surgery. 2012;94:1211-1216; discussion 1216-1217

133. Dujardin KS, Enriquez-Sarano M, Rossi A, Bailey KR, Seward JB. Echocardiographic assessment of left ventricular remodeling: Are left ventricular diameters suitable tools? Journal of the American College of Cardiology. 1997;30:1534-1541

134. Schiller NB, Shah PM, Crawford M, DeMaria A, Devereux R, Feigenbaum H, Gutgesell H, Reichek N, Sahn D, Schnittger I, et al. Recommendations for quantitation of the left ventricle by two-dimensional echocardiography. American society of echocardiography committee on standards, subcommittee on quantitation of two-dimensional echocardiograms. Journal of the American Society of Echocardiography: official publication of the American Society of Echocardiography. 1989;2:358-367

135. Stellbrink C, Breithardt OA, Franke A, Sack S, Bakker P, Auricchio A, Pochet T, Salo R, Kramer A, Spinelli J. Impact of cardiac resynchronization therapy using hemodynamically optimized pacing on left ventricular remodeling in patients with congestive heart failure and ventricular conduction disturbances. Journal of the American College of Cardiology. 2001;38:1957-1965

136. Tei C, Ling LH, Hodge DO, Bailey KR, Oh JK, Rodeheffer RJ, Tajik AJ, Seward JB. New index of combined systolic and diastolic myocardial performance: A simple and reproducible measure of cardiac function-a study in normals and dilated cardiomyopathy. Journal of cardiology. 1995;26:357-366

137. Galasko GI, Basu S, Lahiri A, Senior R. A prospective comparison of echocardiographic wall motion score index and radionuclide ejection fraction in predicting outcome following acute myocardial infarction. Heart. 2001;86:271-276

138. Berning J, Steensgaard-Hansen F. Early estimation of risk by echocardiographic determination of wall motion index in an unselected population with acute myocardial infarction. The American journal of cardiology. 1990;65:567-576

139. Cerqueira MD, Weissman NJ, Dilsizian V, Jacobs AK, Kaul S, Laskey WK, Pennell DJ, Rumberger JA, Ryan T, Verani MS. Standardized myocardial segmentation and nomenclature for tomographic imaging of the heart: A statement for healthcare professionals from the cardiac imaging committee of the council on clinical cardiology of the american heart association. Circulation. 2002;105:539-542

140. Mirsky I, Parmley WW. Assessment of passive elastic stiffness for isolated heart muscle and the intact heart. Circulation research. 1973;33:233-243

141. Aurigemma GP, Silver KH, Priest MA, Gaasch WH. Geometric changes allow normal ejection fraction despite depressed myocardial shortening in hypertensive left ventricular hypertrophy. Journal of the American College of Cardiology. 1995;26:195-202

142. Lancellotti P, Cosyns B, Zacharakis D, Attena E, Van Camp G, Gach O, Radermecker M, Pierard LA. Importance of left ventricular longitudinal function and functional reserve in patients with degenerative mitral regurgitation: Assessment by two-dimensional speckle tracking. Journal of the American Society of Echocardiography: official publication of the American Society of Echocardiography. 2008;21:1331-1336

143. Urheim S, Edvardsen T, Torp H, Angelsen B, Smiseth OA. Myocardial strain by doppler echocardiography. Validation of a new method to quantify regional myocardial function. Circulation. 2000;102:1158-1164

144. Abraham TP, Nishimura RA. Myocardial strain: Can we finally measure contractility? Journal of the American College of Cardiology. 2001;37:731-734

145. Heimdal A, Stoylen A, Torp $H$, Skjaerpe T. Real-time strain rate imaging of the left ventricle by ultrasound. Journal of the American Society of Echocardiography: official publication of the American Society of Echocardiography. 1998;11:1013-1019

146. Henein MY, Gibson DG. Normal long axis function. Heart. 1999;81:111-113 
147. Dumesnil JG, Shoucri RM, Laurenceau JL, Turcot J. A mathematical model of the dynamic geometry of the intact left ventricle and its application to clinical data. Circulation. 1979;59:1024-1034

148. Buckberg GD. Basic science review: The helix and the heart. The Journal of thoracic and cardiovascular surgery. 2002;124:863-883

149. Notomi Y, Setser RM, Shiota T, Martin-Miklovic MG, Weaver JA, Popovic ZB, Yamada H, Greenberg NL, White RD, Thomas JD. Assessment of left ventricular torsional deformation by doppler tissue imaging: Validation study with tagged magnetic resonance imaging. Circulation. 2005;111:1141-1147

150. Hurlburt HM, Aurigemma GP, Hill JC, Narayanan A, Gaasch WH, Vinch CS, Meyer TE, Tighe DA. Direct ultrasound measurement of longitudinal, circumferential, and radial strain using 2-dimensional strain imaging in normal adults. Echocardiography. 2007;24:723-731

151. Azhari H, Weiss JL, Rogers WJ, Siu CO, Zerhouni EA, Shapiro EP. Noninvasive quantification of principal strains in normal canine hearts using tagged mri images in 3-d. The American journal of physiology. 1993;264:H205-H216

152. Rademakers FE, Rogers WJ, Guier WH, Hutchins GM, Siu CO, Weisfeldt ML, Weiss JL, Shapiro EP. Relation of regional cross-fiber shortening to wall thickening in the intact heart. Three-dimensional strain analysis by nmr tagging. Circulation. 1994;89:1174-1182

153. Leitman M, Lysyansky P, Sidenko S, Shir V, Peleg E, Binenbaum M, Kaluski E, Krakover R, Vered Z. Twodimensional strain-a novel software for real-time quantitative echocardiographic assessment of myocardial function. Journal of the American Society of Echocardiography: official publication of the American Society of Echocardiography. 2004;17:1021-1029

154. Reisner SA, Lysyansky P, Agmon Y, Mutlak D, Lessick J, Friedman Z. Global longitudinal strain: A novel index of left ventricular systolic function. Journal of the American Society of Echocardiography: official publication of the American Society of Echocardiography. 2004;17:630-633

155. Langeland S, D'Hooge J, Wouters PF, Leather HA, Claus P, Bijnens B, Sutherland GR. Experimental validation of a new ultrasound method for the simultaneous assessment of radial and longitudinal myocardial deformation independent of insonation angle. Circulation. 2005;112:2157-2162

156. Marwick TH, Leano RL, Brown J, Sun JP, Hoffmann R, Lysyansky P, Becker M, Thomas JD. Myocardial strain measurement with 2-dimensional speckle-tracking echocardiography: Definition of normal range. JACC. Cardiovascular imaging. 2009;2:80-84

157. Brown J, Jenkins C, Marwick TH. Use of myocardial strain to assess global left ventricular function: A comparison with cardiac magnetic resonance and 3-dimensional echocardiography. American heart journal. 2009;157:102 e101-105

158. Carlhall CJ, Nguyen TC, Itoh A, Ennis DB, Bothe W, Liang D, Ingels NB, Miller DC. Alterations in transmural myocardial strain: An early marker of left ventricular dysfunction in mitral regurgitation? Circulation. 2008;118:S256-S262

159. Garcia MJ, Thomas JD, Klein AL. New doppler echocardiographic applications for the study of diastolic function. Journal of the American College of Cardiology. 1998;32:865-875

160. Thomas JD, Weyman AE. Echocardiographic doppler evaluation of left ventricular diastolic function. Physics and physiology. Circulation. 1991;84:977-990

161. Ishida Y, Meisner JS, Tsujioka K, Gallo JI, Yoran C, Frater RW, Yellin EL. Left ventricular filling dynamics: Influence of left ventricular relaxation and left atrial pressure. Circulation. 1986;74:187-196

162. Appleton CP, Hatle LK, Popp RL. Relation of transmitral flow velocity patterns to left ventricular diastolic function: New insights from a combined hemodynamic and doppler echocardiographic study. Journal of the American College of Cardiology. 1988;12:426-440

163. Masuyama T, Lee JM, Yamamoto K, Tanouchi J, Hori M, Kamada T. Analysis of pulmonary venous flow velocity patterns in hypertensive hearts: Its complementary value in the interpretation of mitral flow velocity patterns. American heart journal. 1992;124:983-994

164. Gelsomino S, Lorusso R, Bille G, Rostagno C, De Cicco G, Romagnoli S, Porciani C, Tetta C, Stefano P, Gensini GF. Left ventricular diastolic function after restrictive mitral ring annuloplasty in chronic ischemic mitral regurgitation and its predictive value on outcome and recurrence of regurgitation. International journal of cardiology. 2009;132:419-428 
165. Cianciulli TF, Saccheri MC, Lax JA, Bermann AM, Ferreiro DE. Two-dimensional speckle tracking echocardiography for the assessment of atrial function. World journal of cardiology. 2010;2:163-170

166. Tigen K, Karaahmet T, Dundar C, Guler A, Cevik C, Basaran O, Kirma C, Basaran Y. The importance of papillary muscle dyssynchrony in predicting the severity of functional mitral regurgitation in patients with non-ischaemic dilated cardiomyopathy: A two-dimensional speckle-tracking echocardiography study. European journal of echocardiography: the journal of the Working Group on Echocardiography of the European Society of Cardiology. 2010;11:671-676

167. Calafiore AM, Di Mauro M, Gallina S, Di Giammarco G, laco AL, Teodori G, Tavarozzi I. Mitral valve surgery for chronic ischemic mitral regurgitation. The Annals of thoracic surgery. 2004;77:1989-1997

168. Magne J, Girerd N, Senechal M, Mathieu P, Dagenais F, Dumesnil JG, Charbonneau E, Voisine P, Pibarot P. Mitral repair versus replacement for ischemic mitral regurgitation: Comparison of short-term and long-term survival. Circulation. 2009;120:S104-S111

169. Alfieri O, Maisano F, De Bonis M, Stefano PL, Torracca L, Oppizzi M, La Canna G. The double-orifice technique in mitral valve repair: A simple solution for complex problems. The Journal of thoracic and cardiovascular surgery. 2001;122:674-681

170. Bhudia SK, McCarthy PM, Smedira NG, Lam BK, Rajeswaran J, Blackstone EH. Edge-to-edge (alfieri) mitral repair: Results in diverse clinical settings. The Annals of thoracic surgery. 2004;77:1598-1606

171. Menicanti L, Di Donato M, Frigiola A, Buckberg G, Santambrogio C, Ranucci M, Santo D. Ischemic mitral regurgitation: Intraventricular papillary muscle imbrication without mitral ring during left ventricular restoration. The Journal of thoracic and cardiovascular surgery. 2002;123:1041-1050

172. Kron IL, Green GR, Cope JT. Surgical relocation of the posterior papillary muscle in chronic ischemic mitral regurgitation. The Annals of thoracic surgery. 2002;74:600-601

173. de Varennes B, Chaturvedi R, Sidhu S, Cote AV, Shan WL, Goyer C, Hatzakorzian R, Buithieu J, Sniderman A. Initial results of posterior leaflet extension for severe type iiib ischemic mitral regurgitation. Circulation. 2009;119:2837-2843

174. Hvass $U$, Joudinaud T. The papillary muscle sling for ischemic mitral regurgitation. The Journal of thoracic and cardiovascular surgery. 2010;139:418-423

175. Fukamachi K, Inoue M, Popovic ZB, Doi K, Schenk S, Nemeh H, Ootaki Y, Kopcak MW, Jr., Dessoffy R, Thomas JD, Bianco RW, Berry JM, McCarthy PM. Off-pump mitral valve repair using the coapsys device: A pilot study in a pacing-induced mitral regurgitation model. The Annals of thoracic surgery. 2004;77:688-692; discussion 692-693

176. Livi U, Alfieri O, Vitali E, Russo C, Frigerio M, Tursi V, Albanese MC, De Bonis M, Fragasso G, FrancoCereceda A, Forssell G, Rorke R, Kubo SH. One-year clinical experience with the acorn corcap cardiac support device: Results of a limited market release safety study in italy and sweden. Italian heart journal: official journal of the Italian Federation of Cardiology. 2005;6:59-65

177. Bartkowiak M, Bugajski P, Jedlinski I, Kalawski R. Mitral valve repair in a patient with previous percutaneous annuloplasty with a carillon device. Interactive cardiovascular and thoracic surgery. 2011;12:1054-1056

178. Baldus S, Schillinger W, Franzen O, Bekeredjian R, Sievert H, Schofer J, Kuck KH, Konorza T, Mollmann H, Hehrlein C, Ouarrak T, Senges J, Meinertz T. Mitraclip therapy in daily clinical practice: Initial results from the german transcatheter mitral valve interventions (trami) registry. European journal of heart failure. 2012;14:1050-1055

179. Adams DH, Anyanwu A. Pitfalls and limitations in measuring and interpreting the outcomes of mitral valve repair. The Journal of thoracic and cardiovascular surgery. 2006;131:523-529

180. Magne J, Senechal M, Mathieu P, Dumesnil JG, Dagenais F, Pibarot P. Restrictive annuloplasty for ischemic mitral regurgitation may induce functional mitral stenosis. Journal of the American College of Cardiology. 2008;51:1692-1701

181. Zhu F, Otsuji Y, Yotsumoto G, Yuasa T, Ueno T, Yu B, Koriyama C, Hamasaki S, Biro S, Kisanuki A, Minagoe S, Levine RA, Sakata R, Tei C. Mechanism of persistent ischemic mitral regurgitation after annuloplasty: Importance of augmented posterior mitral leaflet tethering. Circulation. 2005;112:I3961401 
182. Gelsomino S, Lorusso R, Capecchi I, Rostagno C, Romagnoli S, Bille G, De Cicco G, Tetta C, Stefano P, Gensini GF. Left ventricular reverse remodeling after undersized mitral ring annuloplasty in patients with ischemic regurgitation. The Annals of thoracic surgery. 2008;85:1319-1330

183. Penicka M, Linkova H, Lang O, Fojt R, Kocka V, Vanderheyden M, Bartunek J. Predictors of improvement of unrepaired moderate ischemic mitral regurgitation in patients undergoing elective isolated coronary artery bypass graft surgery. Circulation. 2009;120:1474-1481

184. ten Brinke EA, Klautz RJ, Tulner SA, Verwey HF, Bax JJ, Delgado V, Holman ER, Schalij MJ, van der Wall EE, Braun J, Versteegh MI, Dion RA, Steendijk P. Clinical and functional effects of restrictive mitral annuloplasty at midterm follow-up in heart failure patients. The Annals of thoracic surgery. 2010;90:1913-1920 



\section{Chapter 2}

Importance of anterior leaflet tethering in predicting recurrence of ischemic mitral regurgitation after restrictive annuloplasty

Leen Van Garsse, MD, Sandro Gelsomino, MD, PhD, Fabiana Lucà, MD, Roberto Lorusso, MD, PhD, Carmelo Massimiliano Rao, MD, Pieluigi Stefàno, MD, Jos Maessen, $\mathrm{MD}, \mathrm{PhD}$.

Published in: J Thorac Cardiovasc Surg. 2012 Apr;143(4 Suppl):S54-9. 


\section{Abstract}

Objective: We investigated the relationship between anterior mitral (AML) tethering and recurrent ischemic mitral regurgitation (MR) after restrictive annuloplasty. We also explored whether the effect of AML tethering was secondary to modifications in left ventricular size and geometry.

Methods: The study population consisted of 435 consecutive patients with chronic ischemic mitral regurgitation who survived combined coronary artery bypass grafting (CABG) and undersized mitral ring annuloplasty (UMRA) performed at three Institutions (University Hospital, Maastricht, the Netherlands; Careggi Hospital, Florence, Italy; Civic Hospital, Brescia, Italy) between 2001 and 2010. Median follow up was 44.7 months (Interquartile range 25.9 - 66.4). Patients were divided by baseline measurements into quintiles of AML tethering angle $\alpha^{\prime}$ as follows: Group 1, normal/slight AML tethering; Group 2, mild AML tethering; Group 3, moderate AML tethering; Group 4, moderate-severe AML tethering; Group 5, severe AML tethering.

Results: Recurrence of MR was significantly higher in patients with moderate-severe $(28.3 \%)$ and severe $(39.4 \%) A M L$ tethering $(p<0.001)$. There was a strong correlation between $\alpha^{\prime}(r=0.83, p<0.001)$ and recurrent MR whereas there was a weak correlation with posterior mitral angle $\beta^{\prime}(r=0.12, p=0.05)$. At logistic regression analysis corrected for other echocardiographic risk factors, AML tethering $\geq$ moderate-severe (adjusted $\mathrm{OR}, 3.6$ [95\% Cl: $3.0-4.1], \mathrm{p}<0.001$ ) was a strong predictor of MR recurrence. Compared with patients $\beta^{\prime} \geq 45$ those with severe and moderate-severe AML tethering had $>3.7$ and 1.7 times higher odds of MR recurrence, respectively. There were no significant interactions between $\alpha^{\prime}$ and indices of left ventricular function and geometry.

Conclusions: Preoperative AML tethering $\geq$ moderate-severe was strongly associated with recurrence of MR. Assessment of leaflet tethering should be incorporated into clinical risk assessment and prediction models.

\section{Ultramini abstract}

In this multicenter study, we investigated whether a specific tethering pattern is related to higher postoperative recurrence of ischemic MR after CABG and UMRA. Pronounced preoperative anterior mitral leaflet tethering proved to be a powerful predictor of MR recurrence after UMRA independently of LV geometry and LV dilatation. 


\section{Introduction}

Despite Undersized Mitral Ring Annuloplasty (UMRA) being considered effective for chronic ischemic mitral regurgitation $(\mathrm{CIMR})^{1}$ ongoing dissatisfaction with mitral regurgitation (MR) recurrence has been reported predominantly related to continued adverse left ventricular remodeling and ensuing worsening of leaflet tethering ${ }^{2}$.

More recently, attention has been drawn to the preoperative tethering pattern to predict MR recurrence. Nonetheless, published data are conflicting ${ }^{3-5}$ and it is still unclear whether a specific preoperative leaflet configuration is related to unfavorable outcomes.

In this multicenter study, we investigated the relationship between anterior mitral leaflet $(A M L)$ tethering characteristics and postoperative MR recurrence.

\section{Methods}

Ethical Committee approval was waived due to the retrospective analysis of the study according to National laws regulating observational retrospective studies (Italian law nr.11960, released on 13/7/2004; Dutch WMO law). However, all patients gave their informed consent to access their data for scientific purposes.

The study population consisted of 435 consecutive patients with chronic ischemic mitral regurgitation who survived combined coronary artery bypass grafting (CABG) and UMRA performed at three Institutions (University Hospital, Maastricht, the Netherlands; Careggi Hospital, Florence, Italy; Civic Hospital, Brescia, Italy;) between 2001 and 2010. Definition, inclusion and exclusion criteria were as previously reported ${ }^{5}$. Median follow up was 44.7 months (Interquartile range 25.9 -66.4).

All patients underwent complete revascularization. The ring size was determined by standard measurements of the inter-trigonal distance and anterior leaflet height. $A$ downsizing by two ring sizes was performed in all patients. A successful repair was assessed as leaflet coaptation of $0.8 \mathrm{~cm}$ or more, MR of 1 or less, and systolic MV area exceeding $2 \mathrm{~cm}^{2}$ at intraoperative transesophageal echocardiography.

\section{Echocardiographic studies}

Examinations were performed using a commercially available echocardiographic system (IE 33, Philips Medical System, Amsterdam, The Netherlands). A transthoracic echocardiogram (TTE) was carried out before surgery and was repeated annually. All examinations were performed by experienced echocardiographers and stored on a magneto-optical disc. Standard measurements and calculations, quantification of MR and papillary muscle (PM) displacement were carried out as previously reported ${ }^{5}$. 
The AML tethering angle $\alpha^{\prime}$ and the posterior mitral leaflet (PML) angle $\beta^{\prime}$ were directly measured with a specific software (Philips DICOM Viewer, Philips Medical System, Amsterdam, The Netherlands). The excursion angles $\alpha_{\text {ex }}^{\prime}$ and $\beta_{\text {ex }}^{\prime}$ were calculated as the difference between $A M L$ and $P M L$ angles in systole and diastole. The anterior/posterior tethering angle ratio $\alpha^{\prime} / \beta^{\prime}$ was a quantitative measurement of tethering: the more this ratio was approaching 1 the more symmetrical was the tethering. Measurements were made off line by two cardiologists (F.L. and C.M.R.) blinded to the aim of the study. The Cohen method ${ }^{6}$ showed excellent agreement between intra-observer and inter-observer measurements with a concordance (intra-observer 1, intraobserver 2 and inter-observer) of $0.97,0.98$ and 0.96 for $\alpha^{\prime} ; 0.94,0.95$ and 0.92 for $\beta^{\prime}$ and $0.95,0.98$ and 0.94 for coaptation height $(\mathrm{h})$ measured in 20 randomly selected patients. The primary endpoint was the recurrence of $M R$ at latest echocardiographic control. This was defined as insufficiency $\geq 2+$ in patients with no/trivial MR at discharge.

\section{Patient classification}

Patients were divided by baseline measurements into quintiles of AML tethering angle $\alpha^{\prime}$ as follows: Group 1, normal/slight AML tethering, $\alpha^{\prime}<29.8^{\circ}$; Group 2, mild AML tethering, $29.8^{\circ} \geq \alpha^{\prime}<33.4^{\circ}$; Group 3, moderate AML tethering, $33.4^{\circ} \geq \alpha^{\prime}<36.9^{\circ}$; Group 4, moderate-severe AML tethering, 36.9 $\geq \alpha^{\prime}<40.1^{\circ}$; Group 5, severe AML tethering, $\alpha^{\prime}>40.1^{\circ}$.

Patient characteristics are summarized in Table 1 . No difference was found in baseline demographics and operative variables between Groups. In contrast, significant differences were found in echocardiographic parameters. Indeed, subjects with AML tethering $\geq$ moderate-severe had preoperatively larger and more spherical LV ventricles and worse LV function (all, $p<0.001$ ). Furthermore these patients showed a more symmetrical tethering $(p=0.001)$, a lower $A M L$ excursion angle $(p=0.03)$, a more accentuated anterolateral papillary muscle (ALPM) displacement either in lateral $(p=0.004)$ or posterior $(p<0.001)$ direction, a larger $P M s$ separation $(p=0.01)$ and a more accentuated ALPM-Wall Motion score Index (WMSI) $(p<0.001)$. 
Table 1. Patient profile $(n=435)$

\begin{tabular}{|c|c|c|c|c|c|c|}
\hline & $\begin{array}{c}\text { Group } 1 \\
\text { Normal /Slight } \\
\alpha^{\prime}<29.8^{\circ}\end{array}$ & $\begin{array}{c}\text { Group } 2 \\
\text { Mild } \\
\alpha^{\prime} \geq 29.8^{\circ}<33.4^{\circ}\end{array}$ & $\begin{array}{c}\text { Group 3 } \\
\text { Moderate } \\
\alpha^{\prime} \geq 33.4^{\circ}<36.9^{\circ}\end{array}$ & $\begin{array}{c}\text { Group } 4 \\
\text { Moderate-severe } \\
\alpha^{\prime} \geq 36.9^{\circ}<40.1^{\circ}\end{array}$ & $\begin{array}{c}\text { Group } 5 \\
\text { Severe } \\
\alpha^{\prime}>40.1^{\circ} .\end{array}$ & $\mathrm{p}$ \\
\hline Age, $y$ & $65.8 \pm 6.2$ & $66.1 \pm 7.4$ & $66.0 \pm 6.9$ & $66.5 \pm 6.7$ & $67.1 \pm 7.0$ & $\overline{0.8}$ \\
\hline Gender M/F & & & & & & 0.007 \\
\hline Male & $51(58.6)$ & $52(59.7)$ & $50(57.5)$ & $52(59.7)$ & $54(62.0)$ & \\
\hline Female & $36(41.4)$ & $35(40.3)$ & $37(42.5)$ & $35(40.3)$ & $33(38.0)$ & \\
\hline NYHA class & $3[3-4]$ & $3[3-4]$ & $3[3-4]$ & $3[3-4]$ & $3[3-4]$ & $>0.9$ \\
\hline CCS angina class & $2[1-3]$ & $2[1-3]$ & $2[1-3]$ & $2[1-3]$ & $2[1-3]$ & $>0.9$ \\
\hline \multicolumn{7}{|l|}{ Euroscore } \\
\hline Additive & $8.0[6-9]$ & $8.2[7-9]$ & $8.2[7-9]$ & $8 . .4[7-10]$ & $8.7[7-10]$ & 0.08 \\
\hline Logistic & $15.0[12-18]$ & $14.8[12-18]$ & $14.9[12-18]$ & $15.2[12-18]$ & $15.5[12-19]$ & 0.1 \\
\hline Hypertension & $30(36.3)$ & $32(36.7)$ & $39(44.8)$ & $44(39.0)$ & $40(45.9)$ & 0.006 \\
\hline \multicolumn{7}{|l|}{ Myocardial infarction } \\
\hline Inferior/Posterior & $45(51.8)$ & $48(55.2)$ & $42(48.3)$ & $44(50.6)$ & $48(55.2)$ & \\
\hline Anterior/Septal & $8(9.2)$ & $5(5.8)$ & $10(11.4)$ & $6(6.9$ & $4(4.6)$ & 0.53 \\
\hline Lateral & $6(6.9)$ & $5(5.8)$ & $8(9.2)$ & $7(8.0)$ & $4(4.6)$ & \\
\hline Combined & $28(32.1)$ & $29(33.2)$ & $27(31.1)$ & $30(34.5)$ & $31(35.6)$ & \\
\hline \multicolumn{7}{|l|}{ Surgery } \\
\hline CPB time (min) & 111 [94-119] & 105 [95-120] & $109[98-115]$ & $110[100-126]$ & $110[100-130]$ & 0.09 \\
\hline $\mathrm{CCL}$ time (min) & $82[60-98]$ & $85[63-100]$ & 87 [64-99] & 91 [70-106] & 96 [70-108] & 0.06 \\
\hline \multicolumn{7}{|l|}{ Miral Ring } \\
\hline${ }^{\dagger}$ Carpentier Classic & $56(64.3)$ & $60(68.9)$ & $58(66.6)$ & $56(64.3)$ & $61(70.1)$ & 0.75 \\
\hline${ }^{\dagger}$ Physio & $31(35.7)$ & $27(31.1)$ & $29(33.4)$ & $29(35.7)$ & $26(29.9)$ & \\
\hline Ring size (mm) & $28[26-30]$ & $28[26-30]$ & $28[26-30]$ & $28[26-30]$ & $28[26-30]$ & $>0.9$ \\
\hline \multicolumn{7}{|l|}{ CABG } \\
\hline Anastomoses/patient & $2[2-3]$ & $2[2-3]$ & $2[2-3]$ & $2[2-3]$ & $2[2-3]$ & $>0.9$ \\
\hline Arterial graft/patient & $1[1-2]$ & $1[1-2]$ & $1[1-2]$ & $1[1-2]$ & $1[1-2]$ & $>0.9$ \\
\hline \multicolumn{7}{|l|}{ Mitral Regurgitation } \\
\hline MR (grade) & $3[3-4]$ & $3[3-4]$ & $3[3-4]$ & $3[3-4]$ & $3[3-4]$ & $>0.9$ \\
\hline $\operatorname{ERO}\left(\mathrm{mm}^{2}\right)$ & $35 \pm 9$ & $36 \pm 10$ & $38 \pm 12$ & $38 \pm 12$ & $39 \pm 12$ & 0.5 \\
\hline RF (\%) & $45 \pm 12$ & $46 \pm 12$ & $47 \pm 13$ & $46 \pm 13$ & $47 \pm 12$ & 0.5 \\
\hline $\mathrm{RV}$ (ml/beat) & $55 \pm 13$ & $57 \pm 14$ & $58 \pm 15$ & $58 \pm 13$ & $59 \pm 15$ & 0.7 \\
\hline $\mathrm{TA}\left(\mathrm{cm}^{2}\right)$ & $3.2 \pm 1.0$ & $3.2 \pm 1.0$ & $3.4 \pm 1.1$ & $3.9 \pm 1.1^{*}$ & $4.3 \pm 1.1^{*}$ & $<0.001$ \\
\hline $\mathrm{CL}(\mathrm{mm})$ & $3.4 \pm 0.2$ & $3.5 \pm 0.2$ & $3.5 \pm 0.2$ & $4.0 \pm 0.3$ & $3.9 \pm 0.3$ & 0.064 \\
\hline $\mathrm{d}(\mathrm{mm})$ & $35.1 \pm 6.7$ & $35.7 \pm 7.4$ & $35.9 \pm 6.8$ & $34.7 \pm 6.5$ & $37.9 \pm 6.8$ & 0.51 \\
\hline $\mathrm{h}(\mathrm{mm})$ & $10.4 \pm 3.3$ & $10.2 \pm 3.4$ & $10.9 \pm 3.5$ & $11.9 \pm 3.7^{*}$ & $13.8 \pm 4.2^{*}$ & 0.03 \\
\hline \multicolumn{7}{|l|}{ Mitral Leaflet Tethering } \\
\hline$\beta^{\prime}\left({ }^{\circ}\right)$ & $50 \pm 10$ & $54 \pm 12$ & $50 \pm 12$ & $50 \pm 12$ & $50 \pm 10$ & 0.79 \\
\hline$\alpha^{\prime} / \beta^{\prime}$ & $0.59 \pm 0.5$ & $0.59 \pm 0.5$ & $0.68 \pm 0.5$ & $0.78 \pm 0.5^{*}$ & $0.80 \pm 0.8$ & 0.001 \\
\hline$\alpha_{\mathrm{EX}}^{\prime}\left({ }^{\circ}\right)$ & $35.9 \pm 4.6$ & $36.9 \pm 5.2$ & $33.1 \pm 4.5$ & $29.3 \pm 3.2^{*}$ & $28.0 \pm 3.1^{*}$ & $0 . .03$ \\
\hline$\beta_{E X}^{\prime}\left(^{\circ}\right)$ & $14.5 \pm 3.7$ & $14.0 \pm 4.0$ & $14.6 \pm 4.9$ & $13.9 \pm 4.0$ & $13.0 \pm 4.0$ & 0.36 \\
\hline \multicolumn{7}{|l|}{ LV Remodeling } \\
\hline $\mathrm{ESD}(\mathrm{mm})$ & $46 \pm 5$ & $50 \pm 7$ & $46 \pm 6$ & $53 \pm 8^{*}$ & $55 \pm 9^{*}$ & $<0.001$ \\
\hline $\operatorname{EDD}(\mathrm{mm})$ & $57 \pm 6$ & $59 \pm 8$ & $55 \pm 7$ & $65 \pm 7^{*}$ & $67 \pm 9^{*}$ & $<0.001$ \\
\hline $\mathrm{ESV}(\mathrm{ml})$ & $107 \pm 20$ & $111 \pm 22$ & $110 \pm 23$ & $141 \pm 25^{*}$ & $164 \pm 24^{*}$ & $<0.001$ \\
\hline $\operatorname{EDV}(\mathrm{ml})$ & $174 \pm 23$ & $166 \pm 23$ & $171 \pm 28$ & $190 \pm 29^{*}$ & $201 \pm 26^{*}$ & $<0.001$ \\
\hline $\mathrm{SI}_{\mathrm{s}}$ & $0.55 \pm 0.1$ & $0.56 \pm 0.1$ & $0.55 \pm 0.1$ & $0.76 \pm 0.1^{*}$ & $0.82 \pm 0.1^{*}$ & $<0.001$ \\
\hline $\mathrm{SI}_{\mathrm{D}}$ & $0.60 \pm 0.2$ & $0.62 \pm 0.1$ & $0.62 \pm 0.1$ & $0.80 \pm 0.1^{*}$ & $0.88 \pm 0.1^{*}$ & $<0.001$ \\
\hline MPI & $0.52 \pm 0.1$ & $0.64 \pm 0.1$ & $0.66 \pm 0.1$ & $0.95 \pm 0.1^{*}$ & $1.01 \pm 0.3^{*}$ & $<0.001$ \\
\hline
\end{tabular}




\begin{tabular}{|c|c|c|c|c|c|c|}
\hline & $\begin{array}{c}\text { Group } 1 \\
\text { Normal /Slight } \\
\alpha^{\prime}<29.8^{\circ}\end{array}$ & $\begin{array}{c}\text { Group } 2 \\
\text { Mild } \\
\alpha^{\prime} \geq 29.8^{\circ}<33.4^{\circ}\end{array}$ & $\begin{array}{c}\text { Group } 3 \\
\text { Moderate } \\
\alpha^{\prime} \geq 33.4^{\circ}<36.9^{\circ}\end{array}$ & $\begin{array}{c}\text { Group } 4 \\
\text { Moderate-severe } \\
\alpha^{\prime} \geq 36.9^{\circ}<40.1^{\circ}\end{array}$ & $\begin{array}{c}\text { Group } 5 \\
\text { Severe } \\
\alpha^{\prime}>40.1^{\circ} .\end{array}$ & $p$ \\
\hline \multicolumn{7}{|l|}{ Papillary Muscle } \\
\hline \multicolumn{7}{|l|}{ Displacement } \\
\hline \multicolumn{7}{|l|}{ Posterior Displacement } \\
\hline $\operatorname{ALPM}(\mathrm{cm})$ & $2.4 \pm 0.3$ & $2.5 \pm 0.3$ & $2.6 \pm 0.3$ & $3.3 \pm 0.5^{*}$ & $3.3 \pm 0.5^{*}$ & $<0.001$ \\
\hline PMPM (cm) & $2.6 \pm 0.5$ & $2.5 \pm 0.5$ & $2.5 \pm 0.5$ & $2.5 \pm 0.5$ & $2.5 \pm 0.4$ & 0.87 \\
\hline \multicolumn{7}{|l|}{ Lateral Displacement } \\
\hline ALPM $(\mathrm{cm})$ & $1.3 \pm 0.2$ & $1.2 \pm 0.3$ & $1.2 \pm 0.3$ & $1.5 \pm 0.4^{*}$ & $1.6 \pm 0.4^{*}$ & 0.004 \\
\hline PMPM (cm) & $2.0 \pm 0.4$ & $1.9 \pm 0.3$ & $1.9 \pm 0.3$ & $2.0 \pm 0.3$ & $2.0 \pm 0.4$ & 0.89 \\
\hline PMs separation $(\mathrm{cm})$ & $3.3 \pm 0.4$ & $3.3 \pm 0.4$ & $3.4 \pm 0.4$ & $3.7 \pm 0.6$ & $3.6 \pm 0.6^{*}$ & 0.01 \\
\hline \multicolumn{7}{|l|}{ WMSI } \\
\hline ALPM & $1.2 \pm 0.1$ & $1.3 \pm 0.2$ & $1.3 \pm 0.2$ & $1.5 \pm 0.4^{*}$ & $1.5 \pm 0.4^{*}$ & $<0.001$ \\
\hline PMPM & $2.0 \pm 0.3$ & $2.0 \pm 0.3$ & $2.0 \pm 0.3$ & $2.1 \pm 0.3$ & $2.0 \pm 0.3$ & 0.9 \\
\hline
\end{tabular}

Continuous variable are presented as mean \pm standard deviation; Discrete variables are presented as percentage. Non-normal variables are presented as median [Interquartile range]. Abbreviations: Groups, M/F = Male/Female; NYHA = New York Heart Association; CCS = Canadian Cardiovascular Society; CPB = Cardiopulmonary bypass; $\mathrm{CCL}=($ Aortic $)$ Cross-clamp; $\mathrm{CABG}=$ Coronary artery bypass grafting; $\mathrm{MR}=$ Mitral Regurgitation; ERO = effective regurgitant orifice $\left(\mathrm{mm}^{2}\right) ; \mathrm{R}=$ Regurgitant fraction; RV = Regurgitant volume; $\mathrm{TA}=$ Tenting area; $C L=$ Coaptation length; $d=$ coaptation distance; $h=$ coaptation height; $\beta^{\prime}=$ posterior mitral leaflet tethering angle; $\alpha^{\prime}=$ anterior mitral leaflet tethering angle; $\alpha_{\mathrm{EX}}^{\prime}=$ anterior mitral leaflet excursion angle; $\beta_{\mathrm{EX}}^{\prime}=$ posterior mitral leaflet excursion angle; $\mathrm{LV}=$ Left ventricle; $E S D=$ End systolic diameter; EDD = End diastolic diameter; $\mathrm{EDV}=$ End diastolic volume; $\mathrm{ESV}=$ End systolic volume; $\mathrm{SI}_{\mathrm{S}}=\mathrm{Systolic}$ Sphericity Index; $\mathrm{SI}_{\mathrm{D}}=$ Diastolic Sphericity Index; $\mathrm{MPI}=$ Myocardial performance index; ALPM = Antero-lateral papillary muscle; PMPM = Postero-medial papillary muscle; PMs = Papillary muscles; $\mathrm{WMSI}=$ Wall motion score Index; $\mathrm{ns}=$ not significant; * significance at post-hoc test vs. Groups 1-3. ' Edwards LifeSciences, Irvine, CA.

\section{Statistical analysis}

Continuous data were expressed as mean \pm standard deviation, non-normal data were presented as median and interquartile range (IQR) and categorical variables as frequencies. Variables were compared across $\alpha^{\prime}$ categories with the ANOVA, Kuskal-Wallis and $\chi 2$ tests with Tukey and Dunn post-hoc tests, when appropriate.

Pearson correlation analysis was used to test for univariable linear relationships between indices of tethering and postoperative MR recurrence (Regurgitant Volume, continuous variable).

Multivariable logistic regression analysis was performed to assess the effect of preoperative tethering on the recurrence of MR. Forty demographic, clinical and echocardiographic parameters were chosen on the basis of our previous experience ${ }^{5}$. To enhance the accuracy of the model, the number of variables was reduced using variable clustering. Model fit for logistic regression was assessed with the HosmerLemeshow statistic and predictive accuracy was assessed by the concordance index c.

For presentation purposes we first analyzed the main effect of preoperative tethering and then we investigated the model adjusted for variables which were recognized as key factors of MR recurrence ${ }^{5,7}$. Internal validation of predictors generated by 
multivariable logistic regression was performed by means of bootstrapping techniques, with 1000 cycles and generation of OR and bias corrected $95 \% \mathrm{Cl}$.

Finally, to assess whether the predictive value of $\alpha^{\prime}$ was secondary to other factors such as abnormal left ventricular function and geometry, we estimated the effect of $\alpha^{\prime}$ and $\beta^{\prime}$ in subgroups which included systolic sphericity index ( $\mathrm{SI}_{s}$, cut-off: 0.7$)$, end systolic volume (ESV, cut-off: $145 \mathrm{ml}$ ), myocardial performance index (MPI, cut-off: 0.9), wall motion score index (WMSI, cut-off: 1.5) and coaptation height (h, cut-off: 11 $\mathrm{mm})^{5,7,8}$. For the PML tethering angle was chosen a cut-off $\geq 45$ degrees $^{4}$. The effect of the AML tethering angle in each of the subgroups was estimated using logistic regression and compared to $\beta^{\prime} \geq 45$. Then we tested for interactions between $\alpha^{\prime}$ and subgroup variables employing multivariate general linear model (GLM).

SPSS 12.0 (SPSS, Chicago, IL, USA) and Stats Direct 2.5.7 (Stats Direct, Sale, UK) were used for these calculations.

\section{Results}

\section{Recurrent mitral regurgitation}

At follow-up, 99 patients (22.7\%) showed recurrent MR: it occurred in $39.4 \%$ patients with severe $(n=39), 28.3 \%$ moderate-severe $(n=28), 15.1 \%$ moderate $(n=15), 11.1 \%$ mild $(n=11)$ and $6.1 \%(n=6)$ normal/slight $A M L$ tethering. Tenting area (TA) was reduced preoperatively in Groups $1-3\left(2.5 \mathrm{~cm}^{2}, 2.7 \mathrm{~cm}^{2}\right.$ and $2.7 \mathrm{~cm}^{2}$, respectively, $\mathrm{p}<0.001)$ whereas it did not change significantly in Groups 4 and $5\left(3.8 \mathrm{~cm}^{2}\right.$ and $3.7 \mathrm{~cm}^{2}$ in Group 4 and 5, respectively, $p<0.001$ vs. Groups $1-3)$. Coaptation length (CL) was $<8$ $\mathrm{mm}$ in all patients in Groups 4-5 (3.9 $\mathrm{mm}$ and $3.6 \mathrm{~mm}$, respectively) and it was lower than in Groups 1-3 $(8.4 \mathrm{~mm}, 8.4 \mathrm{~mm}$ and $8.6 \mathrm{~mm}$, respectively, $p<0.001$ vs. Groups 13). At follow up $90 / 99$ patients with recurrent MR (90.9\%) had asymmetrical tethering with an eccentric jet without difference between Groups ( $p=0.87$ and $p=0.9$, respectively).

\section{Associations with the outcome}

For all subjects there was a strong correlation between anterior mitral leaflet angle and recurrent mitral regurgitation $(r=0.83, p<0.001)$. This correlation was stronger in patients with severe AML tethering $(r=0.95, p<0.001)$ than in those with moderatesevere $(r=0.56, p=0.008)$, moderate $(r=0.55, p=0.01)$, mild $(r=0.50, p=0.03)$ or slight $(r=0.31, p=0.04) A M L$ tethering. In contrast, there was a weak correlation between $P M L$ angle and MR recurrence $(r=0.12, p=0.05)$. Among parameters of leaflet tethering, there was a significant correlation between $\alpha^{\prime} / \beta^{\prime}(r=0.88, p<0.001)$. Furthermore, $a$ good correlation was found between $\alpha_{\text {ex }}^{\prime}(0.66, p=0.02)$ and recurrent MR whereas 
there was a not significant correlation between $\beta_{\text {ex }}^{\prime}$ and MR recurrence $(r=0.09$, $p=0.43$ ).

At multivariable regression analysis corrected by other echocardiographic risk factors, AML tethering $\geq$ moderate-severe (OR 3.6 [3.0-4.0], $p<0.001$ ], symmetrical pattern (OR 3.4 [2.8 -5.0], $\mathrm{p}<0.001$ ) and anterior leaflet excursion angle $<35^{\circ}$ (OR 2.0 [1.52.6], $p=0.007$ ) were strong predictors of recurrent regurgitation ( $H-L=0.7, c=0.7)$. Compared with patients with posterior leaflet tethering $\geq 45$ degrees (adjusted OR 1.07 [0.09- 1.2], $\mathrm{p}=0.073$ ), those with moderate-severe AML tethering (adjusted OR 1.9 [1.52.5], $p=0.044$ ) had 1.7 times higher odds of MR recurrence (Figure $1 \mathrm{~A}$ ). The increase in risk associated with severe AML tethering was > 3.7-fold (OR 4.0 [3.5- 4.6), p<0.001).

The model proved to be reliable (Hosmer-Lemeshow test, $[H-L] p=0.7$ ) and accurate (c-index $[c]=0.8$ ).

When we allowed for interaction between AML tethering angle and other risk factors (Figure $1 \mathrm{~B}-\mathrm{C}$ ) the predictive value of $\alpha^{\prime}$ was significant across a wide spectrum of patients and ORs were always higher compared with $\mathrm{PML} \geq 45$ degrees. This effect occurred also in low-risk subgroups and it was equivalent or generally attenuated in higher-risk patients. There were no significant interaction between $\alpha^{\prime}$ and any of the covariates (all, $\mathrm{p} \geq 0.05$ ).

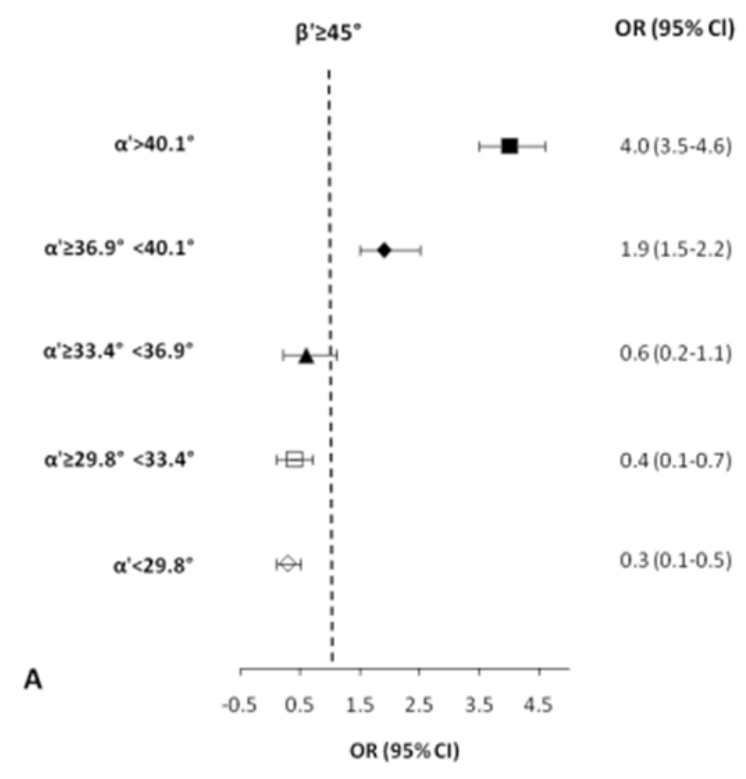




\section{OR vs. PML tethering $\geq 45$ degrees}

Systolic Sphericity Index

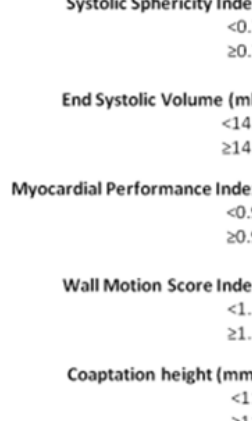

\section{B}
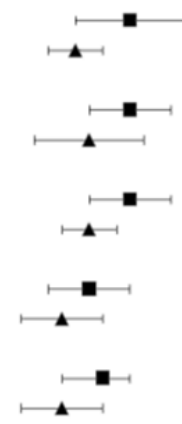

$\geq 11$

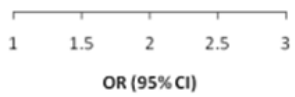

OR vs. PML tethering $\geq 45$ degrees

Systolic Sphericity Index

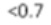

$\geq 0.7$

End Systolic Volume (mI)

$$
<145
$$

$\geq 145$

Myocardial Performance Index

$$
<0.9
$$

$\geq 0.9$

Wall Motion Score Index

$$
<1.5
$$$$
21.5
$$

Coaptation height ( $\mathrm{mm}$ )

$$
\begin{aligned}
& <11 \\
& 211
\end{aligned}
$$

Severe

$\alpha>40.1^{\circ}$

.

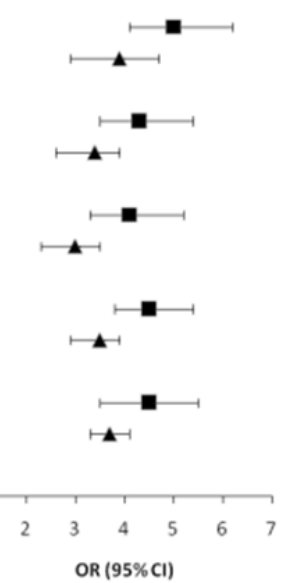

OR $(95 \% \mathrm{Cl})$

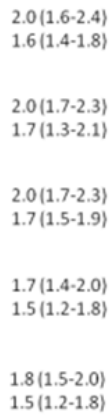

$1.5(1.2-1.8)$

C

$5.0(4.1-6.2)$

$3.9(2.9-4.7)$

$4.3(3.5-5.4)$

$3.4(2.6-3.9)$

$4.1(3.3-5.2)$

$3.0(2.3-3.5)$

4.5 (3.8-5.4)

$3.5(2.9 \cdot 3.9)$

$4.5(3.5-5.5)$

$3.7(3.3-4.1)$

Figure 1. A. Odds ratios (OR) and $95 \%$ confidence interval $(\mathrm{Cl})$ of posterior mitral leaflet tethering angle $\beta^{\prime}$ and different quintiles of anterior mitral leaflet tethering angle $\alpha$ ' in predicting MR recurrence after reductive annuloplasty. B. Sub-groups analysis: odds ratios (OR) and $95 \%$ confidence interval $(\mathrm{Cl})$ of moderatesevere anterior mitral leaflet tethering vs. posterior mitral leaflet tethering angle $\beta^{\prime} \geq 45^{\circ}$ in different subgroups of patients. C. Sub-groups analysis: odds ratios (OR) and $95 \%$ confidence interval (Cl) of severe anterior mitral leaflet tethering vs. posterior mitral leaflet tethering angle $\beta^{\prime} \geq 45^{\circ}$ in different sub-groups of patients. 


\section{Discussion}

This multicenter experience adds to a growing body of literature documenting recurrent ischemic mitral regurgitation after undersized mitral annuloplasty and coronary artery bypass grafting. The present study builds on these findings by examining the real impact of preoperative AML tethering on the recurrence of MR after annuloplasty. Clinical data as well further treatment options offered for patients with discovery of recurrent MR have not been included.

In a previous experience ${ }^{5}$, we have shown that that preoperative $A M L$ tethering angle $\geq 39.5$ degrees had a very high sensitivity and specificity to predict recurrent MR after operation whereas the posterior mitral leaflet (PML) tethering was not significant. Nonetheless, the altered leaflet geometry and recurrent MR could both represent the result of abnormal left ventricular function and geometry which may be primary predictors.

In the present large multicenter study, the predictive value of AML tethering was confirmed. Indeed, compared with patients with posterior leaflet tethering $\geq 45$ degrees, those with moderate-severe AML tethering had 1.7 times higher odds of MR recurrence. The increase in risk associated with severe AML tethering was > 3.7-fold. Furthermore, when we allowed for interaction between $\alpha$ and other risk factors, severe $A M L$ tethering was associated with increased recurrence of $M R$ also in patients with $\mathrm{SI}_{\mathrm{S}}<0.7, \mathrm{ESV}<145 \mathrm{ml} / \mathrm{m}^{2}, \mathrm{MPI}<0.9, \mathrm{WMSI}<1.5$ and $\mathrm{h}<11 \mathrm{~mm}$. This effect was equivalent or generally attenuated in higher-risk patients with significant effect of severe AML tethering on outcomes also in low-risk subgroups. Also, we failed to find any interaction between $\alpha^{\prime}$ and other variables and this demonstrates that AML tethering is a primary predictor of MR and its effect is not secondary to left ventricular function and geometry.

Ciarka et al. demonstrated that tethering of both leaflets are associated with recurrent $\mathrm{MR}^{9}$. Nonetheless, the inclusion in the study of 40 patients (37\%) receiving a cardiac support device, makes it difficult to compare their results with those from our study. Furthermore, our findings are in contrast with Kuwahara et al. ${ }^{3}$ and Magne and coworkers ${ }^{4}$ who showed that the PML tethering angle was a primary predictor of MR. Different methods in calculating tethering angle might explain these conflicting results. However, this topic deserves further investigation to establish the true impact of both leaflets tethering on recurrence of MR.

\section{Clinical implications}

The clinical implications of the present study are important because they suggest that patients with MR recurrence after UMRA for ischemic MR can prospectively be identified on the basis of preoperative echocardiograms. Hence, our results show that the procedure is more likely to fail in patients with AML tethering angle $\geq$ moderate- 
severe. In such patients, concomitant or alternative surgery addressing the leaflet tethering should be considered. We recently started to employ, in these patients, the RING plus STRING technique ${ }^{10}$ which combines the annuloplasty with a repositioning of the posterior papillary muscle toward the mid-septal fibrous annulus (or saddle horn) in the loaded beating heart. Apart from eliminating the need of aggressive annular undersizing, this technique should prevent posterior continued remodeling which has been demonstrated to occur after UMRA ${ }^{5,7}$. Furthermore, mitral valve replacement may play a role in these patients with extreme or unfavorable AML tethering. However, long-term data will be necessary to confirm the optimal management in these circumstances.

\section{Study limitations}

The main limitations of the present study are its retrospective nature and the lack of information on myocardial viability. In the present experience we used only 2 rings which are not at all identical, and thus even though we have undersized both rings by 2 sizes, we might have been less restrictive with the Physio ring than with the Classic ring although, in our previous experience ${ }^{7}$ neither ring type nor ring size were predictive of recurrent MR. How LV remodeling and recurrent MR after UMRA are influenced by the viability of the revascularized myocardium is object of an ongoing study. Furthermore, evaluations were based on 2-D echo measurements that rely on image plane and geometric assumptions which may not be valid when myocardial infarction affects ventricular shape. 3-D echo reconstruction of the endocardial surface would eliminate the need for these assumptions.

\section{Conclusions}

Preoperative $A M L$ tethering is a powerful predictor of $M R$ recurrence after UMRA independently of LV geometry and LV dilatation. Assessment of leaflet tethering by 2-D echocardiography should be incorporated into clinical risk assessment and prediction models. 


\section{References}

1. ten Brinke EA, Klautz RJ, Tulner SA, Verwey HF, Bax JJ, Delgado V, Holman ER, Schalij MJ, van der Wall EE, Braun J, Versteegh MI, Dion RA, Steendijk P. Clinical and functional effects of restrictive mitral annuloplasty at midterm follow-up in heart failure patients. The Annals of thoracic surgery. 2010;90:1913-1920

2. Bouma W, van der Horst IC, Wijdh-den Hamer IJ, Erasmus ME, Zijlstra F, Mariani MA, Ebels T. Chronic ischaemic mitral regurgitation. Current treatment results and new mechanism-based surgical approaches. European journal of cardio-thoracic surgery: official journal of the European Association for Cardio-thoracic Surgery. 2010;37:170-185

3. Kuwahara E, Otsuji Y, Iguro Y, Ueno T, Zhu F, Mizukami N, Kubota K, Nakashiki K, Yuasa T, Yu B, Uemura T, Takasaki K, Miyata M, Hamasaki S, Kisanuki A, Levine RA, Sakata R, Tei C. Mechanism of recurrent/persistent ischemic/functional mitral regurgitation in the chronic phase after surgical annuloplasty: Importance of augmented posterior leaflet tethering. Circulation. 2006;114:1529-1534

4. Magne J, Pibarot P, Dagenais F, Hachicha Z, Dumesnil JG, Senechal M. Preoperative posterior leaflet angle accurately predicts outcome after restrictive mitral valve annuloplasty for ischemic mitral regurgitation. Circulation. 2007;115:782-791

5. Gelsomino S, Lorusso R, Caciolli S, Capecchi I, Rostagno C, Chioccioli M, De Cicco G, Bille G, Stefano P, Gensini GF. Insights on left ventricular and valvular mechanisms of recurrent ischemic mitral regurgitation after restrictive annuloplasty and coronary artery bypass grafting. The Journal of thoracic and cardiovascular surgery. 2008;136:507-518

6. Cohen J. A coefficient of agreement for nominal scales. . Educ Psychol Meas 1960;20:37- 46

7. Gelsomino S, Lorusso R, De Cicco G, Capecchi I, Rostagno C, Caciolli S, Romagnoli S, Da Broi U, Stefano $P$, Gensini GF. Five-year echocardiographic results of combined undersized mitral ring annuloplasty and coronary artery bypass grafting for chronic ischaemic mitral regurgitation. European heart journal. 2008;29:231-240

8. Calafiore AM, Di Mauro M, Gallina S, Di Giammarco G, laco AL, Teodori G, Tavarozzi I. Mitral valve surgery for chronic ischemic mitral regurgitation. The Annals of thoracic surgery. 2004;77:1989-1997

9. Ciarka A, Braun J, Delgado V, Versteegh M, Boersma E, Klautz R, Dion R, Bax JJ, Van de Veire N. Predictors of mitral regurgitation recurrence in patients with heart failure undergoing mitral valve annuloplasty. The American journal of cardiology. 2010;106:395-401

10. Langer F, Schafers HJ. Ring plus string: Papillary muscle repositioning as an adjunctive repair technique for ischemic mitral regurgitation. The Journal of thoracic and cardiovascular surgery. 2007;133:247-249 


\section{Chapter 3}

\section{Impact of pre-operative anterior leaflet}

tethering on the recurrence of ischemic mitral regurgitation and the lack of $L V$ reverse remodeling after restrictive annuloplasty

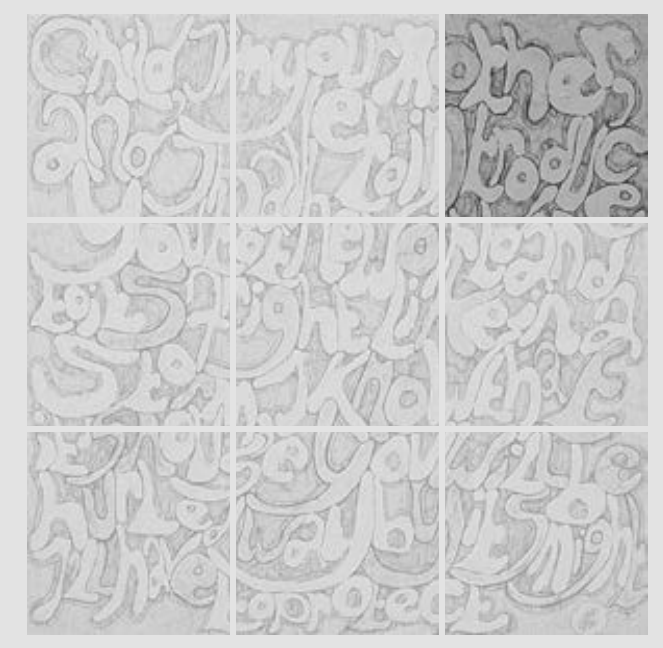

Sandro Gelsomino, MD, PhD, Leen Van Garsse, MD, Fabiana Lucà, MD, Roberto Lorusso, MD, PhD, Emile Cheriex, MD, PhD, Carmelo Massimiliano Rao, MD, Sabina Caciolli, MD, Enrico Vizzardi, MD, Elena Crudeli, MD, Pierluigi Stefàno, MD, Gian Franco Gensini, MD, Jos Maessen, MD, PhD.

Published in: J Am Soc Echocardiogr 2011;24:1365-75. 


\section{Abstract}

Background: In this multicenter study, we investigated the impact of the pre-operative anterior mitral leaflet (AML) tethering angle $\alpha^{\prime}$ on the recurrence of mitral regurgitation (MR) and left ventricular reverse remodeling (LVRR) after undersized mitral ring annuloplasty (UMRA).

Methods: The study population consisted of 362 patients who were divided into two Groups by baseline $\alpha^{\prime}$ : Group 1, $\alpha^{\prime}<39.5^{\circ}(n=196)$; Group 2, $\alpha^{\prime} \geq 39.5^{\circ}(n=166)$. Endpoints were: 1 ) recurrent $M R \geq 2+, 2$ ) LVRR, defined as a reduction in end-systolic volume index $>15 \%$ and 3 ) left ventricular geometrical reverse remodeling, defined as a reduction in systolic sphericity index to a normal value of $<0.72$ in patients with altered baseline geometry.

Results: MR occurred in 9.6\% $(n=19)$ and 43.3\% $(n=72)$ of the patients in Group 1 and Group 2 respectively ( $p<0.001)$. LVRR $(85.7 \%$ vs. $22.2 \%)$ at follow up was higher in Group 1 ( $p<0.001)$. At multivariable regression analysis anterior tethering angle $\alpha^{\prime} \geq$ $39.5^{\circ}$ was a strong predictor of MR recurrence, lack of LV reverse remodeling and lack of LV geometric reverse remodeling (all, $\mathrm{p}<0.001$ ). In contrast the posterior mitral leaflet (PML) tethering angle $\beta^{\prime}$ was not significant (all, $p>0.05$ ). When we allowed for interactions between $\alpha^{\prime}$ and other risk factors this effect occurred also in low-risk subgroups and it was equivalent or generally attenuated in higher-risk patients. There was no significant interaction between $\alpha$ 'and any of the covariates (all, $p>0.05$ ).

Conclusions: AML tethering is a powerful predictor of MR recurrence and lack of LVRR after UMRA. Evaluation of leaflet tethering should be incorporated into clinical risk assessment and prediction models. 


\section{Introduction}

Undersized Mitral Ring Annuloplasty (UMRA) has long been considered as an effective approach to relieve chronic ischemic mitral regurgitation (CIMR). Nonetheless, although few groups report encouraging results after $\mathrm{UMRA}^{1}$, residual/recurrent mitral regurgitation $(\mathrm{MR})$ is seen in up to $30 \%$ of patients in other centers ${ }^{2}$. These disappointing results created the need for a better understanding and preoperative assessment of mitral valve configuration and left ventricular (LV) geometry and function to improve risk stratification and to allow the identification of patient subgroups that are likely to benefit from this procedure.

Recently, great attention has been paid to baseline leaflet configuration. Nonetheless few data are available and published studies show conflicting results ${ }^{3-6}$ regarding a correlation of specific leaflet patterns with unfavorable postoperative outcomes.

Therefore, in this multicenter study, we investigated the impact of AML tethering on the recurrence of mitral regurgitation, left ventricular reverse remodeling (LVRR) and decreased global LV sphericity (left ventricular geometrical reverse remodeling, LVGRR).

\section{Methods}

\section{Ethical issue}

Ethical Committee approval was waived due to the retrospective analysis of the study according to National laws regulating observational retrospective studies (Italian law nr.11960, released on 13/7/2004; Dutch WMO law). However, all patients gave their informed consent to access their data for scientific purposes.

\section{Subjects}

The study population consisted of 391 consecutive patients with CIMR who survived combined coronary artery bypass grafting (CABG) and UMRA performed at three Institutions (Careggi Hospital, Florence, Italy; Civic Hospital, Brescia, Italy; University Hospital, Maastricht, the Netherlands) between October 2008 and April 2010. CIMR was defined as the association of mild-to-severe MR with all the following features: (1) prior myocardial infarction (MI)>16 days; (2) 75\% or greater stenosis of at least one coronary vessel; (3) a corresponding regional wall motion abnormality; (4) type IIIb leaflet dysfunction following Carpentier's classification ${ }^{7}$ with or without annular dilatation.

Twenty-nine patients were excluded: 2 had intraoperative annuloplasty failure, 12 showed residual MR ( $\geq 2+$ at discharge) and 15 had incomplete echoes available. There- 
fore, the final study population consisted of 362 patients. Other exclusion criteria were: 1) degenerative or other non-ischemic etiology; 2) ischemic isolated type I or type II dysfunction ${ }^{7}$; 3) additional mitral valve repair procedures; 4) other valvular or congenital heart diseases; 5 ) previous cardiac surgery or percutaneous coronary angioplasty; 6) atrial fibrillation or sinus rhythm with heart rate at rest $>100$ beats/minute.

One-hundred normal healthy subjects with no history of cardiovascular disease, with normal Doppler echocardiographic examination and who had a gender distribution, age and average body surface area similar to the study patients were controls. Median follow up was 14.3 months (Inter-quartile range 9.3-19.1).

\section{Surgery}

Patients with moderate or severe CIMR (EROA $>20 \mathrm{~mm}^{2}$ and RV $>30 \mathrm{ml}$ ) were scheduled for operation. When MR was 2/4, surgery was indicated: 1 ) in the presence of a dilated LV (end-diastolic volume $>110 \mathrm{~mL} / \mathrm{m}^{2}$ ) or low EF $(<0.35)$, as in the case of dilated cardiomyopathy; 2) in patients with an increase of EROA $>13 \mathrm{~mm}^{2}$ at transthoracic echocardiographic exercise test; 3 ) in ischemic patients with fluctuating MR showing a $M R \geq 3$ after intraoperative loading test.

All patients underwent associated CABG. For the purposes of this study, complete revascularization was accomplished when at least one graft was placed distal to an approximately $50 \%$ diameter narrowing in each of the three major vascular system in which arterial narrowing of this severity was noted in a vessel $\geq 1.5 \mathrm{~mm}$ of diameter. It was not considered necessary to bypass all obstructed diagonal branches of the anterior descending or marginal branches of the circumflex coronary arteries for a classification of complete revascularization. Following this definition $100 \%$ patients underwent complete revascularization. The ring size was determined by standard measurements of the inter-trigonal distance and anterior leaflet height. A downsizing by two ring sizes was performed in all patients.

\section{Echocardiographic measurements}

Two-dimensional and Doppler transthoracic echocardiography examinations were performed using a commercially available echocardiographic system (IE 33, Philips Medical System, Amsterdam, The Netherlands). The clinical echocardiographic evaluation was as follows: a transthoracic echocardiogram (TTE) and a transesophageal echocardiogram (TEE) were performed within 5 days before surgery and serial TTE were performed annually thereafter. Echo examinations were carried out by experienced echocardiographers (S.C., E.V., E.C.) and stored on a magneto-optical disc for off-line analysis. Measurements and calculations were made off line by to cardiologists (F.L and C.M.R.) blinded to the aims of the study. The reliability of echocardiographic measurements was assessed by calculating inter-observer and intra-observer intervals 
of agreement of main direct measures used in this study in 20 subjects randomly chosen among the study patients (Table 1 ).

Table 1. Bland -Altman limits of agreement for intra-observer and inter-observer variability.

\begin{tabular}{lllll}
\hline Variable & & $\begin{array}{l}\text { Mean } \\
\text { difference }\end{array}$ & $\begin{array}{l}\text { Standard } \\
\text { deviation }\end{array}$ & $\begin{array}{l}\text { 95\% limits } \\
\text { of agreement }\end{array}$ \\
\hline$\alpha^{\prime}\left(^{\circ}\right)$ & Intra-observer (FL) & 1.2 & 0.9 & $-2.2-3.1$ \\
& Intra-observer(CMR) & 1.2 & 1.0 & $-1.8-3.2$ \\
& Inter observer & 1.6 & 1.2 & $-2.3-4.0$ \\
$\beta^{\prime}\left({ }^{\circ}\right)$ & Intra-observer (FL) & 1.5 & 0.9 & $-2.0-4.4$ \\
& Intra-observer(CMR) & 1.5 & 1.1 & $-2.8-4.9$ \\
& Inter observer & 1.9 & 1.5 & $-3.5-5.3$ \\
$\mathrm{~h}(\mathrm{~mm})$ & Intra-observer (FL) & 0.1 & 0.3 & $-0.2-0.5$ \\
& Intra-observer(CMR) & 0.2 & 0.1 & $-0.4-0.8$ \\
& Inter observer & 0.2 & 0.1 & $-0.3-0.7$ \\
\hline
\end{tabular}

Intra-observer and inter-observer relative differences were $<5 \%$ for all parameters. The Bland-Altman method showed excellent agreement between intra-observer and inter-observer measurements in both low and high values of echocardiographic parameters. Observers: observer 1: Fabiana Lucà (FL); observer 2: Carmelo Massimiliano Rao (CMR). Inter-observer (observer 1- observer 2).

Abbreviations: $\alpha^{\prime}=$ Anterior mitral leaflet tethering angle, $\beta^{\prime}=$ Posterior mitral leaflet tethering angle; $h$ : coaptation height.

\section{Mitral regurgitation assessment}

The following quantitative measurements were simultaneously employed to grade the severity of MR: 1) Pulsed-wave Doppler (PW-D) and 2) Proximal Isovelocity Surface Area (PISA). When the evidence from different parameters were congruent, the measurements were averaged allowing calculation of regurgitant volume (RV), regurgitant fraction (RF) and effective regurgitant orifice area (EROA) ${ }^{8}$. When different parameters were contradictory PISA was chosen in case of central jet or in presence of calcific mitral valve/mitral annulus whereas PW-D was preferred when the jet was eccentric or multiple ${ }^{8}$. For each measurement, a minimum of three cardiac cycles were averaged. In patients with no or trivial MR by color Doppler, RV and RF were used as calculated, and ERO was assumed as null. The respective thresholds for mild, moderate and severe MR followed American Society of Echocardiography (ASE) recommendations. When the two methods gave contradictory results, the PISA method was preferred in case of central regurgitant jets or in presence of calcific mitral valve/mitral annulus whereas the pulsed-wave Doppler was chosen in presence of eccentric jets or multiple jets ${ }^{8}$.

Recurrent mitral regurgitation at latest follow up was defined as insufficiency $\geq 2+$ in patients with no/trivial MR at discharge. 


\section{Measurement of leaflet tethering}

Mitral valve configuration was assessed in mid-systole using the parasternal long-axis and 4-chamber views. The AML tethering angle $\alpha^{\prime}$ (Figure $1 \mathrm{~A}$ ), the PML tethering angle $\beta^{\prime}$ (Figure $1 \mathrm{~B}$ ) and the bending angle $\gamma$ (Figure $1 \mathrm{C}$ ) were directly measured with specific software (Philips DICOM Viewer, Philips Medical System, Amsterdam, The Netherlands). The excursion angles $\alpha_{\text {ex }}^{\prime}$ and $\beta_{\text {ex }}^{\prime}$ were calculated as the difference between AML and PML angles in systole and diastole. The anterior/posterior tethering angle ratio $\alpha^{\prime} / \beta^{\prime}$ was a quantitative measurement of tethering: the more this ratio was approaching 1 the more symmetric was the tethering.

The tenting area (TA) was measured by the area enclosed between the annular plane and mitral leaflets from the parasternal long-axis view at mid-systole. The coaptation height $(\mathrm{h})$ was measured as the perpendicular distance between the coaptation point of the mitral leaflets and the line connecting the annular hinge points in the long axis view at end-systole. The coaptation length $(\mathrm{CL})$ was measured as the length of apposition of the anterior and posterior mitral leaflets. The coaptation distance (d) was measured (along the annular plane) from the anterior leaflet attachment to the point of coaptation. 


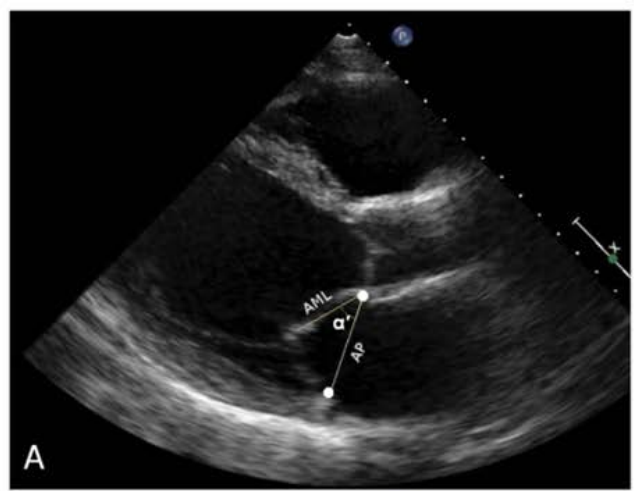

Figure 1. Geometrical mitral valve evaluation in mid-systole. A) The tethering of the anterior mitral leaflet was assessed by measuring the angle $\alpha^{\prime}$ between the annular plane (AP) and the anterior mitral leaflet (AML). B) The tethering of the posterior mitral leaflet was assessed by measuring the angle $\beta^{\prime}$ between the angular plane (AP) and the posterior mitral leaflet (PML). C) The bending angle $\gamma$ was measured as the angle between the bending distance $A B$ ( from the anterior annulus to the bending point $B$ created by the tethering of intermediate or strut chordae in the body of the anterior leaflet) and

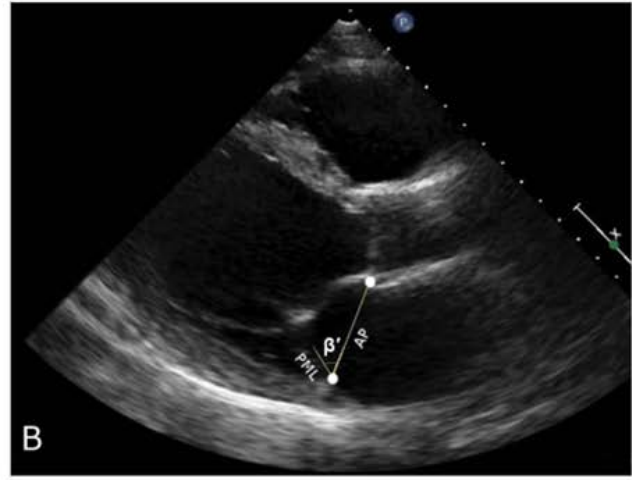
the distance $B C$ from the bending point to the coaptation point $\mathrm{C}$.

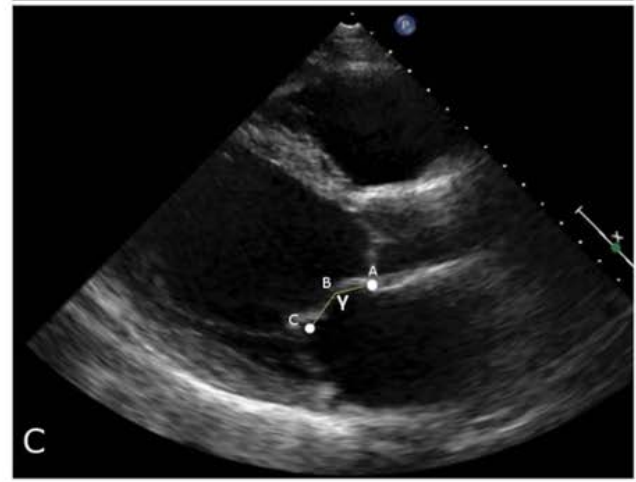

\section{$L V$ remodeling and $L V$ function}

LV volumes and left ventricular ejection fraction (LVEF) were assessed by the bi-apical Simpson disk method ${ }^{9}$. Left Ventricular Reverse Remodeling (LVRR) was defined as a reduction in end-systolic volume index (ESVI) $>15 \%$ at latest echo compared with baseline volume ${ }^{10}$. Sphericity indexes were obtained at end diastole and end systole $\left(\mathrm{SI}_{D}\right.$ and $\mathrm{SI}_{\mathrm{S}}$, respectively) as the volume of the left ventricle divided by the volume of a sphere with a diameter equal to the longest axis of the left ventricle measured in the apical view ${ }^{11}$. Left Ventricular Geometrical Reverse Remodeling (LVGRR) was defined 
as a reduction in $\mathrm{SI}_{\mathrm{S}}$ to a value $<0.72$ (mean value in healthy controls) at latest echocardiogram in patients with altered baseline geometry. The myocardial performance index (MPI) was measured using the method described by Tei et $\mathrm{al}^{12}$.

\section{Papillary muscles (PMs) displacement}

The displacement of papillary muscle was quantified as distances from well-defined anatomic landmarks at early and end systole. From the parasternal short-axis view, the geometric chord defined by the intersection of the right and left ventricles ("septal insertions") and the mid-septal perpendicular line were used as references. Lateral and inferior displacements of anterior and posterior papillary muscles were measured as distances from these fixed references (Figure 2 A-B). Separation between papillary muscles was directly measured.

The lengths between anterolateral papillary muscle (ALPM) and posteromedial papillary muscle (PMPM) tips and the contralateral anterior mitral annulus $\left(I_{1}\right.$ and $I_{2}$, respectively) was also measured in mid-systole in the apical 4- and 2-chamber views by using the anterior mitral annulus as a reference point to estimate outward PM displacement (Figure $2 \mathrm{~B}-\mathrm{C})^{13}$. 


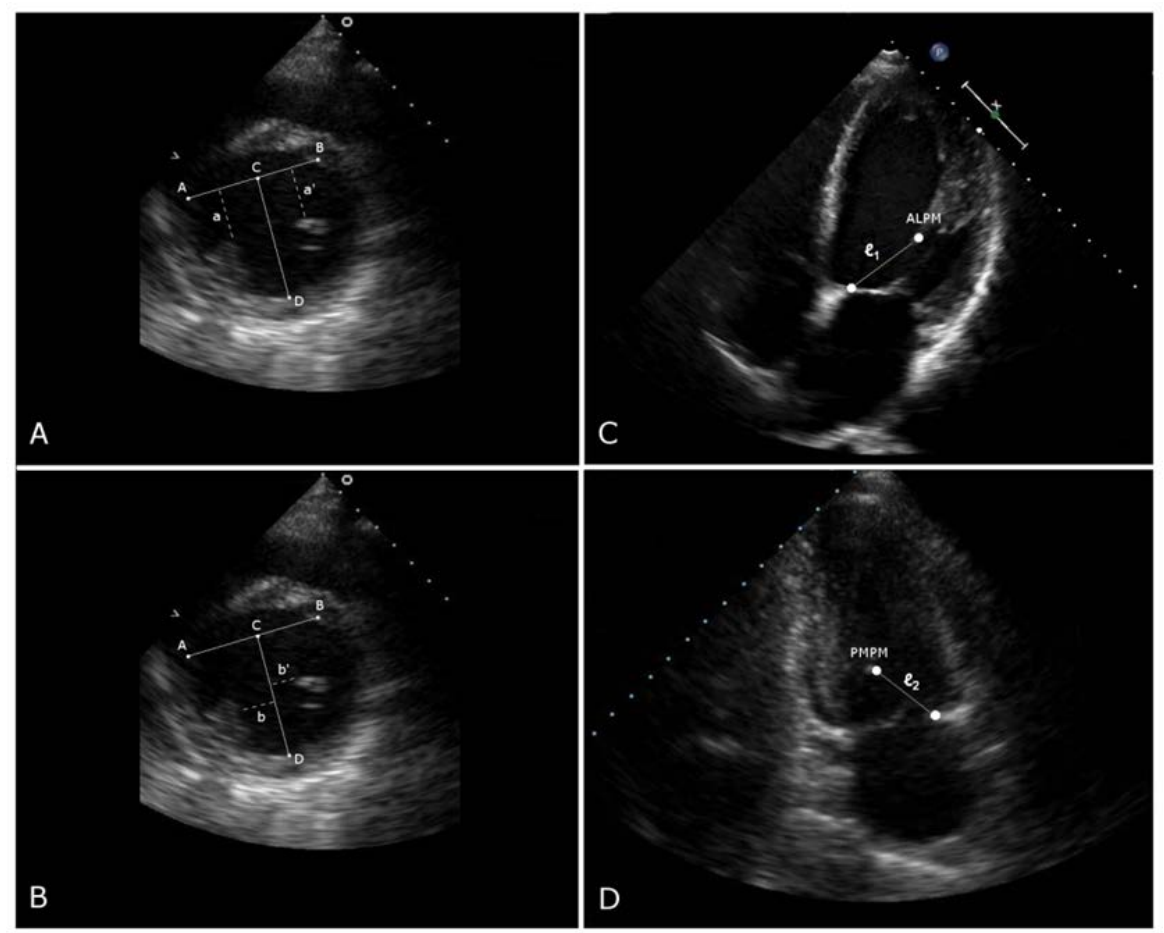

Figure 2. A) Measurement of posterior dislocation of papillary muscles: from the parasternal short-axis view, the geometric chord defined by the intersection of the right and left ventricles (line $A B$ ) and the mid-septal perpendicular line (CD) were used as references. Posterior displacement of anterior and posterior papillary muscles were measured as distances from the line AB. (dashed lines a, a'). B) Measurement of lateral dislocation of papillary muscles: from the parasternal short-axis view, the geometric chord defined by the intersection of the right and left ventricles (line $A B$ ) and the mid-septal perpendicular line (CD) were used as references. Lateral displacement of anterior and posterior papillary muscles were measured as distances from the line $C D$. (dashed lines $b, b^{\prime}$ ). C) Measurement of the length $I_{1}$ between anterolateral papillary muscle (ALPM) and contra lateral anterior mitral annulus. D) Measurement of the length $\mathrm{I}_{2}$ between posterior medial papillary muscle (PMPM) and anterior mitral annulus (see text).

\section{Patient classification}

Patients were divided by baseline measurements of AML tethering angle $\alpha^{\prime}$ into two Groups: Group 1, $\alpha^{\prime}<39.5^{\circ}$ (< severe AML tethering, $\left.n=196\right)$ and Group 2, $\alpha^{\prime} \geq 39.5^{\circ}(\geq$ severe $A M L$ tethering, $n=166$ ). The cut-off was chosen on the basis of our previous experience $^{4}$. For comparisons, a PML tethering angle cut-off $\geq 45$ degrees was chosen ${ }^{5}$.

Patient characteristics are summarized in Table 2. Patients in Group 2 had larger LV diameters and volumes, more spherical ventricles and lower LVEF. No other difference was found in baseline demographics and operative variables between Groups. 
Table 2. Patient profile $(n=362)$

\begin{tabular}{|c|c|c|c|}
\hline & $\begin{array}{c}\text { Group } 1 \\
\alpha^{\prime}<39.5^{\circ} \\
n=196\end{array}$ & $\begin{array}{c}\text { Group 2 } \\
\alpha^{\prime} \geq 39.5^{\circ} \\
n=166\end{array}$ & $p$ \\
\hline Age, y & $65.5 \pm 6.6$ & $66.0 \pm 8.1$ & 0.9 \\
\hline Gender M/F & $109 / 87(55.6 / 44.4)$ & $90 / 76(54.2 / 45.8)$ & 0.7 \\
\hline NYHA class & $3[3-4]$ & $3[3-4]$ & $>0.9$ \\
\hline CCS angina class & $2[1-3]$ & $2[1-3]$ & $>0.9$ \\
\hline \multicolumn{4}{|l|}{ Euroscore } \\
\hline Additive & $8.3[6-10.8]$ & $9.5[7.3-11.4]$ & 0.08 \\
\hline Logistic & $13.4[9.2-14.9]$ & $15.9[10.2-18.0]$ & 0.07 \\
\hline Hypertension & $72(36.7)$ & $55(40.9)$ & 0.4 \\
\hline Diabetes & $54(27.5)$ & $42(25.3)$ & 0.3 \\
\hline COPD & $24(12.2)$ & $23(13.8)$ & 0.6 \\
\hline Chronic renal disease & $30(15.3)$ & $25(15.0)$ & 0.8 \\
\hline Cerebral vascular disease & $24(12.2)$ & $14(10.2)$ & 0.07 \\
\hline Peripheral vascular disease & $18(9.2)$ & $19(11.4)$ & 0.06 \\
\hline Familiar history & $101(51.5)$ & $90(54.2)$ & 0.4 \\
\hline \multicolumn{4}{|l|}{ Myocardial infarction } \\
\hline Inferior/Posterior & $84(42.8)$ & $60(36.1)$ & \\
\hline Anterior/Septal & $9(4.6)$ & $7(4.2)$ & 0.09 \\
\hline Lateral & $13(6.7)$ & $11(6.7)$ & \\
\hline Combined & 90 (45.9) & $88(53.0)$ & \\
\hline Coronary vessel disease & $2[2-3]$ & $2[2-3]$ & $>0.9$ \\
\hline Left Main & $43(21.9)$ & $34(20.4)$ & 0.8 \\
\hline \multicolumn{4}{|l|}{ Medications } \\
\hline Angiotensin-converting enzyme inhibitors & $165(84.1)$ & $140(84.3)$ & \\
\hline B-adrenergic blockers & $103(52.5)$ & $95(57.2)$ & \\
\hline Long-acting nitrates & $87(44.3)$ & $74(44.5)$ & 0.7 \\
\hline Diuretics & $166(84.6)$ & 146 (87.9) & \\
\hline Calcium antagonists & $29(14.7)$ & $21(12.6)$ & \\
\hline Preoperative IABP & $15(7.6)$ & $19(11.4)$ & 0.06 \\
\hline LVEF & $50 \pm 12$ & $41 \pm 9$ & 0.01 \\
\hline EDD & $57 \pm 6$ & $66 \pm 9$ & 0.02 \\
\hline ESD & $49 \pm 7$ & $56 \pm 9$ & 0.03 \\
\hline ESVI & $41 \pm 6$ & $54 \pm 8$ & 0.009 \\
\hline EDVI & $87 \pm 9$ & $100 \pm 16$ & $<0.001$ \\
\hline $\mathrm{SI}_{\mathrm{S}}$ & $0.68 \pm 0.1$ & $0.75 \pm 0.1$ & $<0.001$ \\
\hline$S I_{D}$ & $0.74 \pm 0.1$ & $0.81 \pm 0.1$ & $<0.001$ \\
\hline \multicolumn{4}{|l|}{ Surgery } \\
\hline CPB time (min) & 106 [89-125] & 118 [97-135] & 0.06 \\
\hline CCL time (min) & 85 [70-106] & 90 [76-121] & 0.06 \\
\hline Mitral Ring size (mm) & $28[26-28]$ & $28[26-28]$ & \\
\hline $24 \mathrm{~mm}$ & $11(5.6)$ & $11(6.6)$ & \\
\hline $26 \mathrm{~mm}$ & $74(37.7)$ & $61(36.8)$ & $>0.9$ \\
\hline $28 \mathrm{~mm}$ & $89(45.5)$ & $74(44.6)$ & \\
\hline $30 \mathrm{~mm}$ & $22(11.2)$ & $20(12.0)$ & \\
\hline \multicolumn{4}{|l|}{ CABG } \\
\hline Anastomoses/patient & $2[2-3]$ & $2[2-3]$ & $>0.9$ \\
\hline Arterial graft/patient & $1[1-2]$ & $1[1-2]$ & $>0.9$ \\
\hline
\end{tabular}


Continuous variables are presented as mean \pm standard deviation. Discrete variables are presented as percentages. Non-parametric variables are presented as median [Interquartile range].

Abbreviations: $\mathrm{M} / \mathrm{F}=$ Male/Female; NYHA = New York Heart Association; $\mathrm{CCS}=$ Canadian Cardiovascular Society; $C O P D=$ Chronic obstructive pulmonary disease; $I A B P=$ Intra-aortic balloon pump; LVEF = left ventricular ejection fraction (\%); $\mathrm{EDD}=$ End-diastolic diameter $(\mathrm{mm}) ; \mathrm{ESD}=$ End-systolic diameter $(\mathrm{mm}) ; \mathrm{ESVI}=$ End-systolic Volume Index; EDVI = End-diastolic Volume Index $\left(\mathrm{ml} / \mathrm{m}^{2}\right) ; \mathrm{SI}_{\mathrm{S}}$ = Systolic sphericity Index; $\mathrm{SI}_{\mathrm{D}}=$ diastolic sphericity Index; $\mathrm{CPB}=$ Cardiopulmonary bypass; $\mathrm{CCL}=($ Aortic) Cross-clamp time; $\mathrm{CABG}=$ Coronary artery bypass grafting.

\section{Statistical analysis}

Variables were tested for normal distribution by the Kolmogorov-Smirnov test. Continuous data were expressed as mean \pm standard deviation; non-normal data were presented as median and interquartile range (IQR) and frequencies as proportions. Variables were compared with t-test, Mann-Whitney and $\chi^{2}$ tests, when appropriate.

Multivariable logistic regression analysis was performed to assess the effect of preoperative AML tethering on end-points. Forty demographic, clinical and echocardiographic parameters were investigated for their predictive value. To enhance the accuracy of the model, the number of variables was reduced using variable clustering ${ }^{14}$ until the number of variables to use as candidate in the regression analysis was $\leq m / 10$ where for binary outcomes $\mathrm{m}$ is the number of patients in the less frequent outcome category ${ }^{15}$. Model fit for logistic regression was assessed with the Hosmer-Lemeshow statistic and predictive accuracy was assessed by the concordance index $c^{16}$.

The model adjusted for variables which were recognized as key factors of MR recurrence ${ }^{17-20}$. Internal validation of predictors generated by multivariable logistic regression was performed by means of bootstrapping techniques, with 1000 cycles and generation of OR and bias corrected $95 \% \mathrm{Cl}$.

Finally, to assess whether the predictive value of $\alpha^{\prime}$ was secondary to other factors such as abnormal left ventricular function and geometry, we estimated the effect of $\alpha^{\prime}$ and $\beta^{\prime}$ in subgroups which included systolic sphericity index $\left(\mathrm{SI}_{\mathrm{S}}\right.$, cut-off 0.8$)$, end systolic volume Index (ESVI, cut-off $45 \mathrm{ml} / \mathrm{m}^{2}$ ), myocardial performance index (MPI, cutoff 0.9 ), wall motion score index (WMS, cut-off 1.5) and coaptation height (h, cut-off $11 \mathrm{~mm}$ ). First, we estimated the effect of AML tethering in each of the subgroups. Then we tested for interactions between $\alpha^{\prime}$ and subgroup variables employing multivariate general linear model (GLM).

SPSS 12.0 (SPSS, Chicago, IL, USA) and Stats Direct 2.5.7 (Stats Direct, Sale, UK) were used for these calculations. 


\section{Results}

\section{Recurrent mitral regurgitation and Leaflet tethering}

Data of mitral regurgitation are shown in Table 3. Patients in Group 1 showed a more asymmetrical pattern with a jet direction which was eccentric in most patients whereas in Group 2 the preoperative jet direction was central in the majority of patients. At latest follow-up, 91 patients (25.1\%) showed recurrent MR: it occurred more frequently in Patients in Group 2 (Figure 3 A-D).

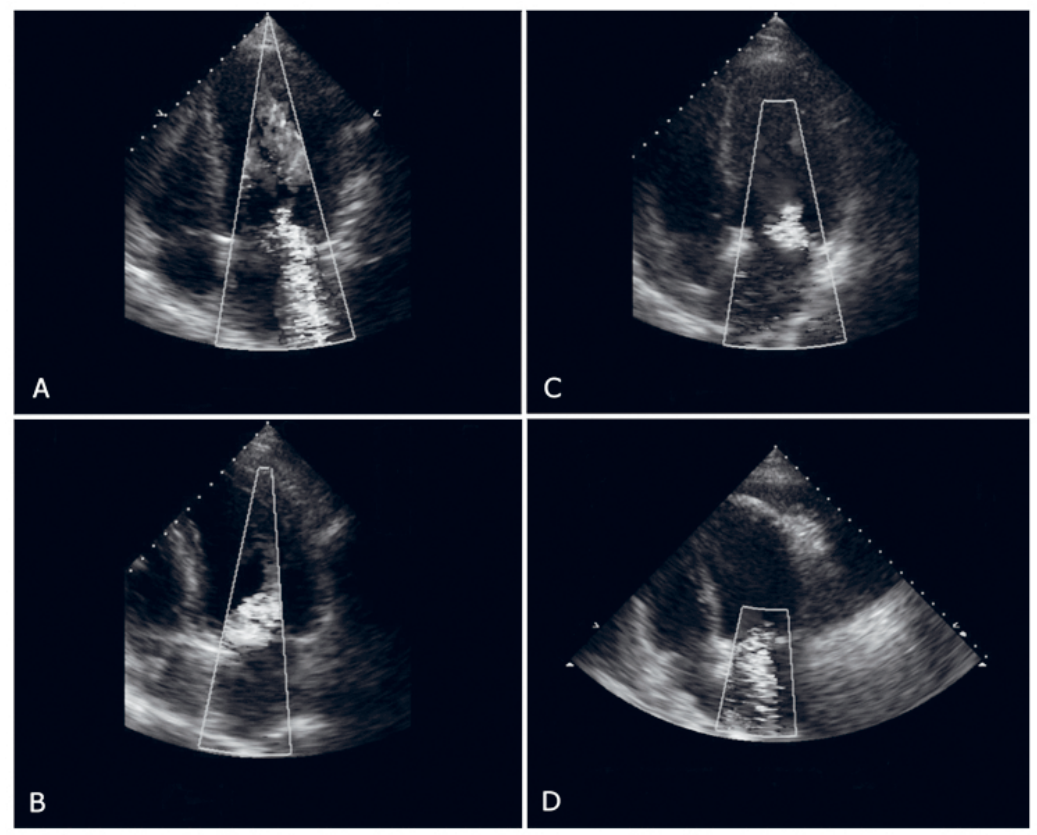

Figure 3. A) Patient belonging to the Group $1\left(\alpha^{\prime}=31.6\right)$ with an eccentric regurgitant jet. B) The same patient as in FIG $3 \mathrm{~A}$ at follow up showed no recurrent mitral regurgitation. C) Patient belonging to the Group 2 $\left(\alpha^{\prime}=41.1\right)$ with a central regurgitant jet. D) Recurrent MR occurred more frequently in Patients in Group 2. The figure shows the same patient as in FIG 3 C at follow up with recurrent mitral regurgitation. The anterior leaflet tethering angle $\alpha^{\prime}$ significantly decreased still remaining higher in Group $2(p=0.001)$, whereas the excursion angle $\alpha_{\text {ex }}^{\prime}$ increased in both Groups, but it was significantly lower in Group 2 ( $\left.p=0.03\right)$. In contrast, the posterior leaflet tethering angle $\beta^{\prime}$ increased in both groups whereas the excursion angle $\beta^{\prime}{ }_{\text {ex }}$ reduced significantly in both Groups without any significant difference between them. Finally, the bending angle $\gamma$ increased significantly in Group 1 whereas it did not change significantly in Group 2. 
Table 3. Mitral Regurgitation ( $n=362)$

\begin{tabular}{|c|c|c|c|c|c|c|c|}
\hline & \multirow[t]{2}{*}{$\begin{array}{c}\text { Controls } \\
n=100\end{array}$} & \multicolumn{3}{|c|}{$\begin{array}{c}\text { Group } 1 \\
\alpha^{\prime}<39.5^{\circ} \\
n=196\end{array}$} & \multicolumn{3}{|c|}{$\begin{array}{c}\text { Group } 2 \\
\alpha^{\prime} \geq 39.5^{\circ} \\
n=166\end{array}$} \\
\hline & & Preoperative & Follow up & $\mathrm{p}$ & Preoperative & Follow up & $\mathrm{p}$ \\
\hline \multicolumn{8}{|l|}{$\mathrm{MR}$} \\
\hline Grade & $0[0-1]$ & $3[3-4]^{*}$ & $0[0-1]$ & $<0.001$ & $3[3-4]^{*}$ & $2[1-2]^{*+}$ & 0.002 \\
\hline None & $62(62.0)$ & - & $161(82.1)$ & & - & $25(15.1)$ & \\
\hline $1+$ & $38(38.0)$ & - & $16(8.2)$ & & - & $69(41.5)$ & \\
\hline $2+$ & - & $29(14.7)$ & $16(8.2)$ & & $26(15.7)$ & $52(31.4)$ & \\
\hline $3+$ & - & $122(62.4)$ & $3(1.5)$ & & $100(60.7)$ & $11(6.6)$ & \\
\hline $4+$ & - & 45 (22.9) & - & & $40(24.0)$ & $9(5.4)$ & \\
\hline \multicolumn{8}{|c|}{ Direction of regurgitant jet } \\
\hline Central & - & $17(8.7)$ & - & $<0.001$ & $104(62.7)^{+}$ & $1(1.4)$ & \\
\hline Anterior & - & $21(10.7)$ & - & & $15(9.0)$ & $1(1.4)$ & $<0.001$ \\
\hline Posterior & - & $131(66.8)$ & $17(89.4)$ & & $21(12.7)^{+}$ & $68(94.4)$ & \\
\hline Complex & - & $27(13.8)$ & $2(10.6)$ & & $26(15.6)$ & $2(2.8)$ & \\
\hline $\operatorname{ERO}\left(\mathrm{mm}^{2}\right)$ & - & $35.3 \pm 10.2$ & - & - & $39.4 \pm 11.7$ & $27.7 \pm 10.3$ & 0.05 \\
\hline RF (\%) & - & $43.3 \pm 9.4$ & - & - & $46.6 \pm 13.4$ & $32.4 \pm 12.6$ & 0.04 \\
\hline $\mathrm{RV}$ (ml/beat) & - & $54.9 \pm 11.7$ & - & - & $57.1 \pm 14.6$ & $38.2 \pm 13.4$ & 0.02 \\
\hline $\mathrm{TA}\left(\mathrm{cm}^{2}\right)$ & $0.8 \pm 0.2$ & $3.2 \pm 1.1^{*}$ & $1.9 \pm 0.3^{*}$ & $<0.001$ & $4.2 \pm 1.1^{*+}$ & $2.8 \pm 1^{*+}$ & $<0.001$ \\
\hline $\mathrm{CL}(\mathrm{mm})$ & $8.2 \pm 0.3$ & $4.0 \pm 0.6^{*}$ & $8.6 \pm 0.3$ & $<0.001$ & $3.2 \pm 0.3^{*+}$ & $4.0 \pm 0.3^{* \dagger}$ & 0.8 \\
\hline $\mathrm{d}(\mathrm{mm})$ & $28.2 \pm 7.0$ & $35.4 \pm 7.3^{*}$ & $31.4 \pm 5.6^{*}$ & 0.43 & $36.8 \pm 6.1^{*}$ & $38.7 \pm 8.4^{* \dagger}$ & 0.65 \\
\hline $\mathrm{h}(\mathrm{mm})$ & $7.0 \pm 3.1$ & $10.0 \pm 1.9^{*}$ & $8.3 \pm 0.8$ & 0.03 & $13.2 \pm 2.2^{* \dagger}$ & $11.7 \pm 1.6^{*+}$ & 0.2 \\
\hline \multicolumn{8}{|c|}{ Mitral Leaflet Tethering } \\
\hline$\beta^{\prime}\left({ }^{\circ}\right)$ & $34.2 \pm 6.2$ & $52.0 \pm 10.1^{*}$ & $60.3 \pm 12.1^{*}$ & $<0.001$ & $50.6 \pm 12.2^{*}$ & $60.1 \pm 14.4^{*}$ & $<0.001$ \\
\hline$\alpha^{\prime}\left({ }^{\circ}\right)$ & $23.9 \pm 3.1$ & $33.8 \pm 4.4^{*}$ & $26.1 \pm 3.5^{*}$ & $<0.006$ & $40.4 \pm 6.0^{*}$ & $33.0 \pm 4.4$ & 0.01 \\
\hline$\alpha^{\prime} / \beta^{\prime}$ & $0.70 \pm 0.4$ & $0.65 \pm 0.1^{*}$ & $0.50 \pm 0.1^{*}$ & $<0.001$ & $0.80 \pm 0.1^{*+}$ & $0.55 \pm 0.2^{*}$ & $<0.001$ \\
\hline$\gamma\left({ }^{\circ}\right)$ & $152.1 \pm 13.2$ & $140.2 \pm 10.3^{*}$ & $151.1 \pm 11.4$ & 0.02 & $129.3 \pm 6.4^{* \dagger}$ & $121.5 \pm 5.3^{* \dagger}$ & 0.08 \\
\hline$\alpha_{\mathrm{EX}}^{\prime}\left(^{\circ}\right)$ & $42.4 \pm 4.3$ & $36.6 \pm 4.2^{*}$ & $43.5 \pm 4.9$ & 0.02 & $27.2 \pm 3.3^{*+}$ & $38.9 \pm 5.2^{*+}$ & 0.007 \\
\hline$\beta_{\mathrm{EX}}^{\prime}\left({ }^{\circ}\right)$ & $24.9 \pm 3.3$ & $14.2 \pm 2.7^{*}$ & $8.9 \pm 1.2^{*}$ & 0.004 & $13.9 \pm 4.3^{*}$ & $9.2 \pm 1.7^{*}$ & 0.01 \\
\hline
\end{tabular}

Continuous variables are presented as mean \pm standard deviation. Non-parametric variables are presented

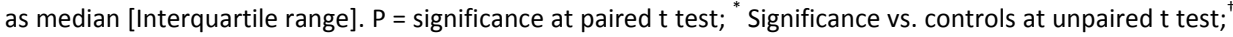
significance vs. Group 1at unpaired t test.

Abbreviations: $M R=$ Mitral Regurgitation; $E R O=$ effective regurgitant orifice; $R F$ = Regurgitant fraction; RV = Regurgitant volume; $\mathrm{TA}=$ Tenting area; $\mathrm{CL}=$ Coaptation length; $\mathrm{d}=$ coaptation distance; $\mathrm{h}=$ coaptation height; $\beta^{\prime}=$ posterior mitral leaflet tethering angle; $\alpha^{\prime}=$ anterior mitral leaflet tethering angle; $\gamma=$ bending angle; $\alpha_{\mathrm{EX}}^{\prime}=$ anterior mitral leaflet excursion angle; $\beta_{\mathrm{EX}}^{\prime}=$ posterior mitral leaflet excursion angle.

\section{Papillary muscle displacement}

As shown in Table 4, patients in Group 1 had higher PMPM apical displacement $(p<0.001)$ whereas patients in Group 2 had preoperatively greater posterior, lateral and apical displacement of ALPM (all, $\mathrm{p}<0.001$ ) and a wider papillary muscle separation $(p<0.001)$. Posterior and lateral displacement of the PLPM were comparable between both Groups.

At follow-up, indices of posterior $(p=0.003)$, lateral $(p=0.002)$ and apical $(p=0.01)$, displacement of the anterior papillary muscle and $\mathrm{PM}_{\mathrm{S}}$ separation $(\mathrm{p}=0.004)$ reduced significantly from the preoperative value only in Groups 1 , whereas all remained stable 
in patients in Group 2 with values significantly higher (all, $p<0.001$ ). Indexes of posterior $(p=0.001, p<0.001)$ and lateral $(p=0.003$ and $p<0.001)$ displacement of the PMPM increased significantly in both Groups at follow-up whereas apical displacement of PMPM did not change in both Groups.

Table 4. Papillary Muscle Displacement $(n=362)$

\begin{tabular}{|c|c|c|c|c|c|c|c|}
\hline & \multirow[t]{2}{*}{$\begin{array}{l}\text { Controls } \\
n=100\end{array}$} & \multicolumn{3}{|c|}{$\begin{array}{c}\text { Group } 1 \\
\alpha^{\prime}<39.5^{\circ}\end{array}$} & \multicolumn{3}{|c|}{$\begin{array}{c}\text { Group 2 } \\
\alpha^{\prime} \geq 39.5^{\circ} \\
n=166\end{array}$} \\
\hline & & Preoperative & Follow up & $p$ & Preoperative & Follow up & $p$ \\
\hline \multicolumn{8}{|l|}{ Posterior Displacement } \\
\hline $\operatorname{ALPM}(\mathrm{cm})$ & $1.9 \pm 0.2$ & $2.4 \pm 0.3^{*}$ & $2.2 \pm 0.2^{*}$ & 0.003 & $3.2 \pm 0.4^{*+}$ & $3.3 \pm 0.4^{*+}$ & 0.79 \\
\hline PMPM (cm) & $1.8 \pm 0.2$ & $2.6 \pm 0.5^{*}$ & $2.8 \pm 0.3^{*}$ & 0.001 & $2.5 \pm 0.4^{*}$ & $2.8 \pm 0.4^{*}$ & $<0.001$ \\
\hline \multicolumn{8}{|l|}{ Lateral Displacement } \\
\hline $\operatorname{ALPM}(\mathrm{cm})$ & $1.0 \pm 0.2$ & $1.3 \pm 0.2^{*}$ & $1.1 \pm 0.1^{*}$ & 0.002 & $1.6 \pm 0.3^{*+}$ & $1.6 \pm 0.3^{* \dagger}$ & 0.9 \\
\hline $\operatorname{PMPM}(\mathrm{cm})$ & $1.3 \pm 0.2$ & $2.0 \pm 0.4^{*}$ & $2.4 \pm 0.3^{*}$ & 0.003 & $2.0 \pm 0.4^{*}$ & $2.4 \pm 0.4^{*}$ & $<0.001$ \\
\hline \multicolumn{8}{|l|}{ Apical Displacement } \\
\hline $\operatorname{ALPM}\left(I_{1}, \mathrm{~cm}\right)$ & $3.3 \pm 0.2$ & $3.5 \pm 0.2^{*}$ & $3.3 \pm 0.2^{*}$ & 0.01 & $3.7 \pm 0.4^{*+}$ & $3.7 \pm 0.4^{*+}$ & 0.9 \\
\hline $\operatorname{PMPM}\left(\mathrm{I}_{2}, \mathrm{~cm}\right)$ & $3.3 \pm 0.2$ & $3.6 \pm 0.4^{*}$ & $3.6 \pm 0.2^{*}$ & 0.89 & $3.4 \pm 0.3^{*+}$ & $3.4 \pm 0.3^{*}$ & 0.89 \\
\hline PMs separation $(\mathrm{cm})$ & $2.3 \pm 0.3$ & $3.3 \pm 0.4^{*}$ & $3.1 \pm 0.2^{*}$ & 0.004 & $3.6 \pm 0.6^{*+}$ & $3.7 \pm 0.6^{*+}$ & 0.68 \\
\hline
\end{tabular}

Continuous variables are presented as mean \pm standard deviation. Non-parametric variables are presented as median [Interquartile range]. ${ }^{*}$ Significance vs. controls; ${ }^{\dagger}$ significance vs. Group 1.

Abbreviations: $\mathrm{ALM}=$ Anterolateral papillary muscle; $\mathrm{PMPM}=$ Posteromedial papillary muscle; PMs = Papillary muscles.

\section{$L V$ reverse remodeling, $L V$ geometrical reverse remodeling and $L V$ function}

In patients in Group 1 ESVI decreased significantly at follow up while in Group 2 it increased at latest control (Figure 4 A). Changes in end-diastolic volume index showed the same tendency to ESVI (Figure 4 B). When the degree of LV reverse remodeling at follow up was compared in the two groups (Figure $4 \mathrm{C}$ ) it was significantly higher in Group 1 ( $p<0.001)$.

At follow up, systolic and diastolic sphericity indexes reduced significantly in Group $1\left(S I_{S}, 0.58 \pm 0.1, p<0.001 ; S I_{D}, 0.64 \pm 0.1, p<0.001\right)$, whereas these indexes increased in Group $2\left(\mathrm{SI}_{S}, 0.80 \pm 0.1, \mathrm{p}=0.02 ; \mathrm{SI}_{\mathrm{D}}, 0.88 \pm 0.1, \mathrm{p}=0.01\right)$. The degree of geometrical reverse remodeling at follow up was significantly higher compared in Group 1 (Figure 4 D). 
ESVI

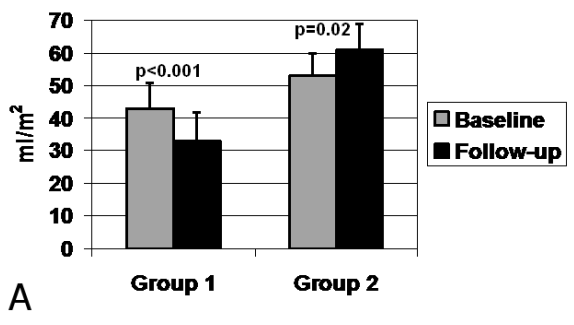

EDVI

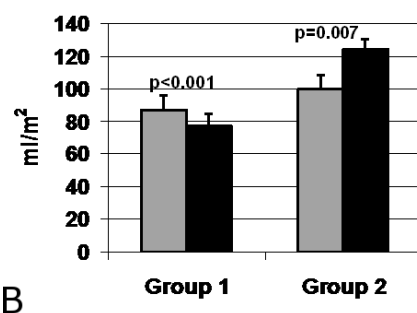

LVRR

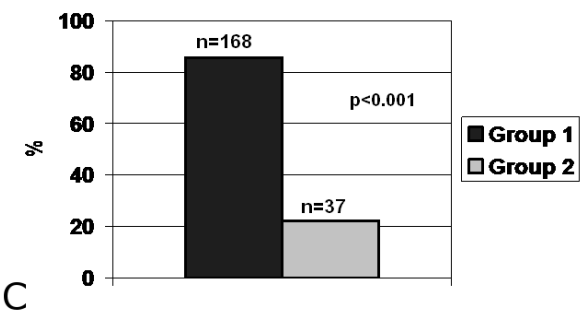

LVGRR

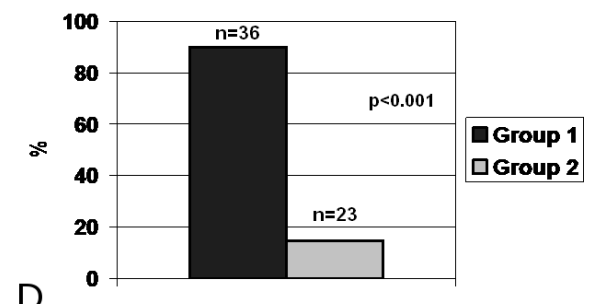

Figure 4. A) End Systolic Volume Index (ESVI) with 95\% confidence Interval (error bars) at baseline and follow up. B) End Diastolic Volume Index (EDVI) with 95\% confidence Interval (error bars) at baseline and follow up. C) Percentage of left Ventricular Reverse Remodeling (LVRR, \%) in the two Groups. D) Left Ventricular Geometrical Reverse Remodeling (LVGRR, \%) defined as reduction in systolic sphericity index to a value $<0.72$ at latest echocardiogram among 40 patients in Group 1 and 158 in Group 2 with altered baseline geometry.

\section{Association of pre-operative AML tethering with MR recurrence and lack of LV reverse remodeling}

At multivariable regression analysis anterior tethering angle $\alpha^{\prime} \geq 39.5^{\circ}$ was a strong negative predictor of MR recurrence, lack of LV reverse remodeling and lack of LV geometric reverse remodeling (Table 5). Compared with patients with posterior leaflet tethering $\geq 45$ degrees, an $\alpha^{\prime} \geq 39.5^{\circ}$ carried a $>3$-fold increase in MR recurrence and $>4$-fold increase in continued remodeling and continued spherical remodeling. Compared with patients with $\alpha^{\prime}<39.5^{\circ}$ the increase in risk associated with severe AML tethering was $>3$-fold for MR recurrence, $>4$ fold for lack of LVRR and >5-fold for lack of LV geometrical remodeling. Models proved to be reliable and accurate (Table 5) .

When we allowed for interactions between $\alpha^{\prime}$ and other risk factors (Figure 5 A-C), the predictive value of $\alpha^{\prime}$ was significant across a wide spectrum of patients. This effect also occurred in low-risk subgroups and it was equivalent or generally attenuated in higher-risk patients. There were no significant interactions between $\alpha^{\prime} \geq 39.5^{\circ}$ and any of the covariates. (all, $p>0.05$ ). 
Table 5. Relationship of tethering angles and major end-points.

\begin{tabular}{lcccccc}
\hline & \multicolumn{2}{c}{$\beta^{\prime} \geq 45^{\circ}$} & \multicolumn{2}{c}{$\alpha^{\prime}<39.5^{\circ}$} & \multicolumn{2}{c}{$\alpha^{\prime} \geq 39.5^{\circ}$} \\
\cline { 2 - 7 } & OR $(95 \% \mathrm{Cl})$ & $\mathrm{p}$ & OR $(95 \% \mathrm{Cl})$ & $\mathrm{p}$ & OR $(95 \% \mathrm{Cl})$ & $\mathrm{p}$ \\
\hline *MR Recurrence & $0.94(0.45-1.42)$ & 0.079 & $0.88(0.31-1.43)$ & 0.009 & $3.06(2.51-3.64)$ & $<0.001$ \\
+Lack of LVRR & $1.03(0.51-1.57)$ & 0.06 & $0.85(0.38-1.40)$ & 0.1 & $4.15(3.59-4.74)$ & $<0.001$ \\
\# Lack of LVGRR & $0.98(0.50-1.52)$ & 0.06 & $0.83(0.37-1.39)$ & 0.36 & $4.80(4.24-5.46)$ & $<0.001$ \\
\hline
\end{tabular}

Abbreviations: $M R=$ Mitral Regurgitation; LVRR = Left ventricular reverse remodeling; LVGRR = Left ventricular geometrical reverse remodeling; $\alpha^{\prime}=$ Anterior mitral leaflet angle; $\beta^{\prime}=$ Posterior mitral leaflet angle $O R=$ Odds Ratio; $\mathrm{Cl}=$ Confidence Interval: Models validation. ${ }^{*}$ Hosmer-Lemeshow test, $[\mathrm{H}-\mathrm{L}]=0.7, \mathrm{C}-\mathrm{index}[\mathrm{c}]=$ $0.8 .{ }^{\dagger} \mathrm{H}-\mathrm{L}=0.9, \mathrm{c}=0.7 ;{ }^{\ddagger} \mathrm{H}-\mathrm{L}=0.8, \mathrm{c}=0.7$.

MR (\%)

$\alpha^{\prime}<39.5^{\circ}$ vs. $\alpha^{\prime} \geq 39.5^{\circ}$

$20(n=168) 5.9$ vs. 22.6 $<0.80$ ( $n=194) 4.6$ vs. 17.5

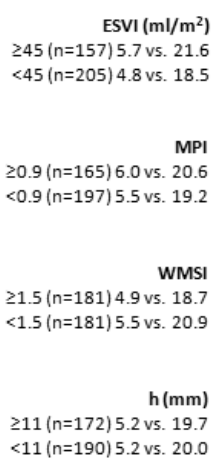

A
OR ( $95 \% \mathrm{Cl})$; P value

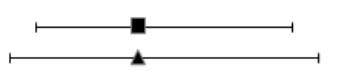

$3.4(3.0-4.0) ;<0.001$ $3.4(2.9-4.1) ;<0.001$

P for interaction with $\alpha^{\prime} \geq 39.5^{\circ}: 0.951$

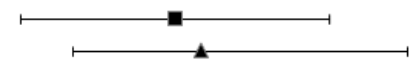

$3.3(2.7-3.9) ;<0.001$ $3.4(2.9-4.2) ;<0.001$

$P$ for interaction with $\alpha^{\prime} \geq 39.5^{\circ}: 0.263$

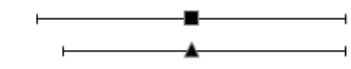

$3.0(2.4-3.6) ;<0.001$ $3.0(2.5-3.6) ;<0.001$

$P$ for interaction with $\alpha^{\prime} \geq 39.5^{\circ}: 0.915$

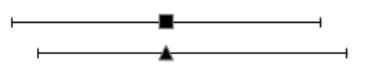

$3.4(2.8-4.0) ;<0.001$ $3.4(2.9-4.1) ;<0.001$

P for interaction with $\alpha^{\prime} \geq 39.5^{\circ}: 0.934$

$3.3(2.7-3.9) ;<0.001$ $3.4(2.9-4.0) ;<0.001$

$P$ for interaction with $\alpha^{\prime} \geq 39.5^{\circ}: 0.243$

\begin{tabular}{llcccc|r}
\hline 1.5 & 2.5 & 3 & 3.5 & 4 & 4.5 \\
& & & & & & \\
& & & &
\end{tabular}




\section{LVRR (\%)}

$\alpha^{\prime}<39.5^{\circ}$ vs. $\alpha^{\prime} \geq 39.5^{\circ}$

OR (95\% CI); P value

SIs
$\geq 0.80(n=168) 45.8$ vs. 10.1
$<0.80(n=194) 46.9$ vs. 10.3
MPI
$\geq 0.9(n=165) 45.4$ vs. 10.3
$<0.9(n=197) 47.2$ vs. 10.1

WMSI
$\geq 1.5$ (n=181) 45.8 vs. 9.9
$<1.5(n=181) 46.9$ vs. 10.4

h (mm)
$<11(n=172) 44.7$ vs. 9.8

B

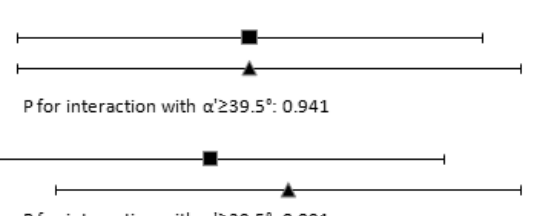

P for interaction with $\alpha^{\prime} \geq 39.5^{\circ}: 0.091$

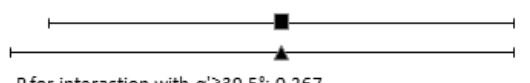

P for interaction with $\alpha^{\prime} \geq 39.5^{\circ}: 0.267$

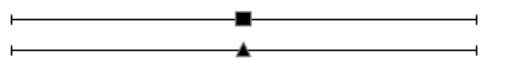

P for interaction with $\alpha^{\prime} \geq 39.5^{\circ}: 0.964$
$4.1(3.5-4.7):<0.001$ $4.1(3.5-4.8) ;<0.001$

$4.0(3.4-4.6) ;<0.001$ $4.2(3.6-4.8) ;<0.001$

$4.2(3.6-4.8) ;<0.001$ $4.2(3.5-4.8) ;<0.001$

$4.1(3.5-4.7) ;<0.001$ $4.1(3.5-4.7) ;<0.001$

\begin{tabular}{|cccc}
\hline 3.5 & 4 & 4.5 & 5 \\
& OR $(95 \% \mathrm{Cl})$ & &
\end{tabular}

LVGRR (\%)

$\alpha^{\prime}<39.5^{\circ}$ vs. $\alpha^{\prime} \geq 39.5^{\circ}$

OR (95\% CI); P value

$\operatorname{ESVI}\left(\mathrm{ml} / \mathrm{m}^{2}\right)$ $\geq 0.80$ ( $n=168) 38.8$ vs. 7.6 $<0.80(n=194) 42.4$ vs. 7.8

MPI
$\geq 0.9(n=165) 43.0$ vs. 9.0 $<0.9(n=197) 39.1$ vs. 6.5 (1)

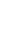

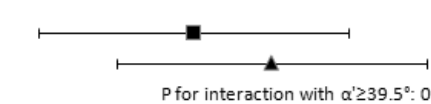

$P$ for interaction with $\alpha^{\prime} \geq 39.5^{\circ}: 0.064$

$\geq 1.5$ ( $n=181$ ) 39.7 v5. 7.7 $<1.5(n=181) 41.9$ vs. 7.7

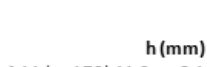

$\geq 11$ ( $n=172$ ) 41.2 vs. 8.1 $<11(n=190) 40.5$ vs. 7.3

C

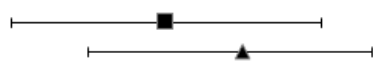

$4.8(4.2-5.4) ;<0.001$

$P$ for interaction with $\alpha^{\prime} \geq 39.5^{\circ}: 0.089$ $5.1(4.5-5.6) ;<0.001$

$4.8(4.2-5.4) ;<0.001$ $5.2(4.7-5.8) ;<0.001$

P for interaction with $\alpha^{\prime} \geq 39.5^{\circ}: 0.106$

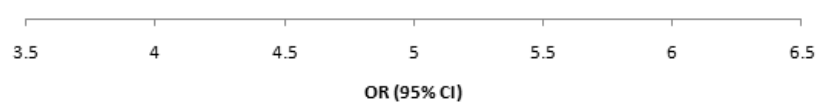

Figure 5. Sub-groups analyses. A) Recurrent Mitral Regurgitation (MR): odds ratios (OR) and $95 \%$ confidence interval $(\mathrm{Cl})$ of $\alpha^{\prime} \geq 39.5^{\circ}$ vs. $\alpha^{\prime}<39.5^{\circ}$ in different sub-groups of patients. B) Left Ventricular Reverse Remodeling (LVRR): odds ratios (OR) and 95\% confidence interval (CI) of $\alpha^{\prime} \geq 39.5^{\circ}$ vs. $\alpha^{\prime}<39.5^{\circ}$ in different sub-groups of patients. C) Left Ventricular Geometric Reverse Remodeling (LVGRR): odds ratios (OR) and $95 \%$ confidence interval $(\mathrm{Cl})$ of $\alpha^{\prime} \geq 39.5^{\circ}$ vs. $\alpha^{\prime}<39.5^{\circ}$ in different sub-groups of patients. 


\section{Discussion}

The mechanism of chronic ischemic mitral regurgitation (CIMR) is complex and involves the left ventricular remodeling with increased sphericity and displacement of both papillary muscles. All of these functional phenomena cause valve leaflet tethering by the outward displacement of the papillary muscles and annular dilatation, resulting in mal-coaptation of the leaflets ${ }^{2}$. CIMR continues to be a complex surgical problem for which a reproducible and reliable surgical correction is yet to be found. Undersized mitral ring annuloplasty (UMRA) has been successfully used for more than a decade and has been accepted as an effective means for immediate improvement in valve function $^{21}$. Despite the enthusiasm for a low perioperative mortality rate, a positive effect on survival and functional symptomatic status, a number of studies have clearly demonstrated that UMRA is subject to variable rates of recurrence ${ }^{2,22}$, related to continued adverse LV remodeling and ensuing worsening of leaflet tethering ${ }^{5,22}$. However, recent studies have confirmed that some CIMR patients may benefit from UMRA ${ }^{1}$, thus the search for preoperative parameters which may predict surgical failure continues.

More recently, attention has been drawn to preoperative leaflet configuration to predict MR recurrence and outcomes. Nonetheless, published data are conflicting ${ }^{3-5}$ and it is still unclear whether a specific leaflet configuration is related to unfavorable outcomes.

The purpose of this multicenter study was to investigate the influence of preoperative leaflet tethering patterns on main outcomes after UMRA. We studied the relation between anterior leaflet tethering and the recurrence of mitral regurgitation. Furthermore we analyzed its influence on postoperative normalization of LV volume and geometry. Indeed, it is well known that the shape of the LV wall plays an important role in the pathophysiology of ischemic cardiomyopathy and CIMR. Thus the attenuation or reversal of geometric LV remodeling is an important aim in the treatment of CIMR. The impact of preoperative AML tethering on main outcomes was compared to that of PML $\geq 45$ degrees which, in previous studies, were shown to predict MR persistence and worse outcomes after restrictive annuloplasty ${ }^{23}$.

Furthermore, the influence of AML tethering could be secondary to altered LV geometry and recurrent MR may represent both the result of abnormal left ventricular function and shape which may be primary predictors. For this purpose, we allowed for interaction between AML tethering angle and other risk factors to explore significant interactions with these variables and to test the false hypothesis that the effect of $A M L$ tethering on main outcomes is increased only in high-risk sub-categories.

The main findings of this study can be summarized as follows:

First, this large multicenter study confirmed our previous experience ${ }^{4}$, demonstrating that preoperative anterior tethering angle $\alpha^{\prime} \geq 39.5^{\circ}$ accurately predicts recurrence of MR after undersized annuloplasty and coronary artery bypass grafting. Indeed, compared with patients with $\alpha^{\prime}<39.5^{\circ}$ the increase in risk associated with severe AML 
tethering was $>3$-fold. The risk was 3.24 times higher compared to those patients with significant $\mathrm{PML}$ tethering (PML angle $\geq 45^{\circ}$ ). In our analysis residual MR was excluded. Persistent/residual MR is presumably due to immediate failure of the technique employed rather than LV remodeling ${ }^{24}$ and it is not surprising that regurgitation persists at late follow up in these patients. This might partly explain different results with published studies that reached different conclusions. Other explanations may be that most of these studies included small numbers of patients and that MR severity was quantitatively evaluated only with the use of vena contracta width and not by PISA or volumetric methods ${ }^{3-5}$.

Second, in our experience $\alpha^{\prime} \geq 39.5^{\circ}$ was a strong independent predictor of lack of left ventricular reverse remodeling (LVRR) as well as left ventricular geometrical reverse remodeling (LVGRR). Patients with severe preoperative AML tethering showed a $>4$ times and $>5$ times higher odds for lack of LVRR and LVGRR, respectively, compared to those with $\alpha^{\prime}<39.5^{\circ}$. Odds were $>4$ higher for both lack of LVRR and LVGRR, compared to those with PML angle $\geq 45$ degrees. However, it is not clear how an "annular solution to a ventricular problem" may influence LV reverse remodeling. Bolling et $\mathrm{al}^{25}$ suggested that the small ring would decrease LV wall stress by remodeling the base of the heart and restoring a more elliptical shape to the LV cavity. He also opined that undersized ring annuloplasty achieves sufficient acute geometrical remodeling to begin a slower process of "auto-remodeling" over time. Power et $\mathrm{al}^{26}$ hypothesized that even a small reduction in LV workload might allow reverse remodeling to occur and showed that even passive ventricular restraint was sufficient to attenuate LV dilation and progressive functional decline in an experimental heart failure model. Takeda et $\mathrm{al}^{27}$ demonstrated that decrease in afterload after reduction in volume overload was responsible for LVRR process after UMRA. To the best of our knowledge, ours is the first study to associate preoperative tethering with postoperative reduction in volume and geometry.

Third, when we allowed for interaction between $\alpha^{\prime} \geq 39.5^{\circ}$ and other risk factors, severe $A M L$ tethering was associated with increased recurrence of MR also in patients with $\mathrm{SI}_{\mathrm{s}}<0.80, \mathrm{ESVI}<45 \mathrm{ml} / \mathrm{m}^{2}, \mathrm{MPI}<0.9, \mathrm{WMSI}<1.5$ and $\mathrm{h}<11 \mathrm{~mm}$. This effect was equivalent or generally attenuated in higher-risk patients and it was observed also in the sub-analysis of LVRR and LVGRR with the significant effect of severe AML tethering on outcomes also in low-risk subgroups. Furthermore, we failed to find any interaction between $\alpha^{\prime} \geq 39.5^{\circ}$ and other variables.

Fourth, patients with severe AML tethering had higher displacement (posterior, lateral and apical) of the ALPM and a higher WMSI at the level of the anterior papillary muscle. It is not clear why AML tethering is associated with ALPM displacement and why ALPM displacement does not affect PML tethering, since the AML is attached by chordae to both ALPM and PMPM. As stated by Nielsen ${ }^{28}$ "Functional MR appears in an orchestra of geometric and hemodynamic variables that accompany acute and chronic LV dysfunction". The number of variables is too high to predict changes that reflect on 
leaflets function in advance ${ }^{28,29}$. We might postulate that this different effect on leaflets is the result of a series of factors ${ }^{28,29}$ such as: 1 ) the absence of 3rd order chordae in AML: the presence of these chordae on PML contributes to its decreased mobility relative to $A M L ; 2$ ) the chordae that tether the $P M L$ are aligned parallel to each other, while the AML chordae are inserted obliquely on either side $s$ of the leaflet; 3) PM chords are shorter $(0.6-1.5 \mathrm{~cm}$ vs. $1.32-2.39 \mathrm{~cm})$ and thicker $(0.11-0.93 \mathrm{~mm}$ vs. $1.41-$ $1.76)$; 4) The unequal size results in a different distribution of tethering forces; 5 ) the variability in number of chordae; 6) Chordal force distribution determines systolic mitral leaflet configuration and the severity of functional MR.

Fifth, in accordance with published data ${ }^{30}$, our findings show that, in the postoperative period, there is an increased PMPM-to-annulus tethering length after PMPM displacement in lateral and posterior direction rather than in apical direction along the left ventricular long axis. The lateral and posterior shift of the papillary muscle redirects papillary muscle tension away from the axial direction diverting the leaflets away from closure.

\section{Clinical implications}

The clinical implications of the present study are important because they suggest that patients with MR recurrence after UMRA for ischemic MR can prospectively be identified on the basis of preoperative echocardiograms. Hence, our results show that the procedure is more likely to fail in patients with $\alpha^{\prime} \geq 39.5^{\circ}$. In such patients, concomitant or alternative surgery addressing the leaflet tethering should be considered. In patients with $\alpha^{\prime} \geq 39.5^{\circ}$ and symmetric tethering we perform UMRA with adjunctive PM repositioning under transesophageal echocardiography guidance in the loaded beating heart using a trans-ventricular suture (STRING). The RING plus STRING procedure $^{31}$ apart from eliminating the need for aggressive annular undersizing, is expected to prevent continued remodeling which is mainly related to failure after UMRA ${ }^{22}$. Despite encouraging preliminary results, long-term data will be necessary to confirm the effectiveness of this procedure.

\section{Study limitations}

The main limitations of the present study are its retrospective nature and the lack of information on myocardial viability. Indeed, both left ventricular remodeling and recurrent MR after mitral annuloplasty would be expected to be influenced by the adequacy of revascularization, the viability of the revascularized myocardium and medical therapy. Complete revascularization was defined from a surgical perspective but we did not have data about myocardial scar and myocardial perfusion following surgery. We are collecting data and these issues will be the subject of an on-going study. Furthermore, postoperative medical therapy (especially afterload-reducing therapy, beta- 
adrenergic antagonists, and diuretic therapy) which may have significantly influenced outcomes, has not been evaluated in our analysis.

Finally echocardiographic evaluations were based on 2-D echo measurements that rely on image plane and geometric assumptions which may not be valid when myocardial infarction affects ventricular shape. Three-D echo reconstruction ${ }^{32}$ of the endocardial surface would eliminate the need for these assumptions.

\section{Conclusions}

Preoperative $A M L$ tethering is a powerful predictor of MR recurrence and left ventricular reverse remodeling after UMRA. Evaluation of leaflet tethering by 2-D echocardiography should be incorporated into clinical risk assessment and prediction models. 


\section{References}

1. ten Brinke EA, Klautz RJ, Tulner SA, Verwey HF, Bax JJ, Delgado V, Holman ER, Schalij MJ, van der Wall $\mathrm{EE}$, Braun J, Versteegh MI, Dion RA, Steendijk P. Clinical and functional effects of restrictive mitral annuloplasty at midterm follow-up in heart failure patients. The Annals of thoracic surgery. 2010;90:1913-1920

2. Bouma W, van der Horst IC, Wijdh-den Hamer IJ, Erasmus ME, Zijlstra F, Mariani MA, Ebels T. Chronic ischaemic mitral regurgitation. Current treatment results and new mechanism-based surgical approaches. European journal of cardio-thoracic surgery: official journal of the European Association for Cardio-thoracic Surgery. 2010;37:170-185

3. Kuwahara E, Otsuji Y, Iguro Y, Ueno T, Zhu F, Mizukami N, Kubota K, Nakashiki K, Yuasa T, Yu B, Uemura T, Takasaki K, Miyata M, Hamasaki S, Kisanuki A, Levine RA, Sakata R, Tei C. Mechanism of recurrent/persistent ischemic/functional mitral regurgitation in the chronic phase after surgical annuloplasty: Importance of augmented posterior leaflet tethering. Circulation. 2006;114:1529-1534

4. Gelsomino S, Lorusso R, Caciolli S, Capecchi I, Rostagno C, Chioccioli M, De Cicco G, Bille G, Stefano P, Gensini GF. Insights on left ventricular and valvular mechanisms of recurrent ischemic mitral regurgitation after restrictive annuloplasty and coronary artery bypass grafting. The Journal of thoracic and cardiovascular surgery. 2008;136:507-518

5. Magne J, Pibarot P, Dumesnil JG, Senechal M. Continued global left ventricular remodeling is not the sole mechanism responsible for the late recurrence of ischemic mitral regurgitation after restrictive annuloplasty. Journal of the American Society of Echocardiography: official publication of the American Society of Echocardiography. 2009;22:1256-1264

6. Lesniak-Sobelga A, Wicher-Muniak E, Kostkiewicz M, Olszowska M, Musialek P, Klimeczek P, Banys P, Pasowicz M, Tracz W, Podolec P. Relationship between mitral leaflets angles, left ventricular geometry and mitral deformation indices in patients with ischemic mitral regurgitation: Imaging by echocardiography and cardiac magnetic resonance. The international journal of cardiovascular imaging. 2012;28:59-67

7. Carpentier A. Cardiac valve surgery--the "french correction". The Journal of thoracic and cardiovascular surgery. 1983;86:323-337

8. Zoghbi WA, Enriquez-Sarano M, Foster E, Grayburn PA, Kraft CD, Levine RA, Nihoyannopoulos P, Otto CM, Quinones MA, Rakowski H, Stewart WJ, Waggoner A, Weissman NJ. Recommendations for evaluation of the severity of native valvular regurgitation with two-dimensional and doppler echocardiography. Journal of the American Society of Echocardiography: official publication of the American Society of Echocardiography. 2003;16:777-802

9. Schiller NB, Shah PM, Crawford M, DeMaria A, Devereux R, Feigenbaum H, Gutgesell $H$, Reichek N, Sahn D, Schnittger I, et al. Recommendations for quantitation of the left ventricle by two-dimensional echocardiography. American society of echocardiography committee on standards, subcommittee on quantitation of two-dimensional echocardiograms. Journal of the American Society of Echocardiography: official publication of the American Society of Echocardiography. 1989;2:358-367

10. Stellbrink C, Breithardt OA, Franke A, Sack S, Bakker P, Auricchio A, Pochet T, Salo R, Kramer A, Spinelli J. Impact of cardiac resynchronization therapy using hemodynamically optimized pacing on left ventricular remodeling in patients with congestive heart failure and ventricular conduction disturbances. Journal of the American College of Cardiology. 2001;38:1957-1965

11. Kono T, Sabbah HN, Rosman H, Alam M, Jafri S, Goldstein S. Left ventricular shape is the primary determinant of functional mitral regurgitation in heart failure. Journal of the American College of Cardiology. 1992;20:1594-1598

12. Tei C, Ling LH, Hodge DO, Bailey KR, Oh JK, Rodeheffer RJ, Tajik AJ, Seward JB. New index of combined systolic and diastolic myocardial performance: A simple and reproducible measure of cardiac function-a study in normals and dilated cardiomyopathy. Journal of cardiology. 1995;26:357-366 
13. Zhang H, Otsuji Y, Uemura T, Yu B, Takeuchi M, Hamasaki S, Miyata M, Kisanuki A, Minagoe S, Levine RA, Tei C. Different mechanisms of ischemic mitral regurgitation in patients with inferior and anterior myocardial infarction. J Echocardiogr. 2008;8:74-83

14. Romesburg H. Cluster analysis for researchers. North Carolina: Lulu Press. 2004:1-330

15. Harrell FE, Jr., Lee KL, Califf RM, Pryor DB, Rosati RA. Regression modelling strategies for improved prognostic prediction. Statistics in medicine. 1984;3:143-152

16. Hosmer DW, Lemeshow S. Applied logistic regression. 2nd ed. New York: Wiley-Interscience. 2000:143202

17. Ciarka A, Braun J, Delgado V, Versteegh M, Boersma E, Klautz R, Dion R, Bax JJ, Van de Veire N. Predictors of mitral regurgitation recurrence in patients with heart failure undergoing mitral valve annuloplasty. The American journal of cardiology. 2010;106:395-401

18. Calafiore AM, Di Mauro M, Gallina S, Di Giammarco G, laco AL, Teodori G, Tavarozzi I. Mitral valve surgery for chronic ischemic mitral regurgitation. The Annals of thoracic surgery. 2004;77:1989-1997

19. Gelsomino S, Lorusso R, De Cicco G, Capecchi I, Rostagno C, Caciolli S, Romagnoli S, Da Broi U, Stefano $P$, Gensini GF. Five-year echocardiographic results of combined undersized mitral ring annuloplasty and coronary artery bypass grafting for chronic ischaemic mitral regurgitation. European heart journal. 2008;29:231-240

20. Kongsaerepong V, Shiota M, Gillinov AM, Song JM, Fukuda S, McCarthy PM, Williams T, Savage R, Daimon M, Thomas JD, Shiota T. Echocardiographic predictors of successful versus unsuccessful mitral valve repair in ischemic mitral regurgitation. The American journal of cardiology. 2006;98:504-508

21. Bax JJ, Braun J, Somer ST, Klautz R, Holman ER, Versteegh MI, Boersma E, Schalij MJ, van der Wall EE, Dion RA. Restrictive annuloplasty and coronary revascularization in ischemic mitral regurgitation results in reverse left ventricular remodeling. Circulation. 2004;110:II103-II108

22. Hung J, Papakostas L, Tahta SA, Hardy BG, Bollen BA, Duran CM, Levine RA. Mechanism of recurrent ischemic mitral regurgitation after annuloplasty: Continued IV remodeling as a moving target. Circulation. 2004;110:1185-II90

23. Magne J, Pibarot P, Dagenais F, Hachicha Z, Dumesnil JG, Senechal M. Preoperative posterior leaflet angle accurately predicts outcome after restrictive mitral valve annuloplasty for ischemic mitral regurgitation. Circulation. 2007;115:782-791

24. Adams DH, Anyanwu A. Pitfalls and limitations in measuring and interpreting the outcomes of mitral valve repair. The Journal of thoracic and cardiovascular surgery. 2006;131:523-529

25. Bach DS, Bolling SF. Improvement following correction of secondary mitral regurgitation in end-stage cardiomyopathy with mitral annuloplasty. The American journal of cardiology. 1996;78:966-969

26. Power JM, Raman J, Dornom A, Farish SJ, Burrell LM, Tonkin AM, Buxton B, Alferness CA. Passive ventricular constraint amends the course of heart failure: A study in an ovine model of dilated cardiomyopathy. Cardiovascular research. 1999;44:549-555

27. Takeda K, Taniguchi K, Shudo Y, Kainuma S, Hamada S, Matsue H, Matsumiya G, Sawa Y. Mechanism of beneficial effects of restrictive mitral annuloplasty in patients with dilated cardiomyopathy and functional mitral regurgitation. Circulation. 2010;122:S3-S9

28. Nielsen SL, Nygaard H, Fontaine AA, Hasenkam JM, He S, Andersen NT, Yoganathan AP. Chordal force distribution determines systolic mitral leaflet configuration and severity of functional mitral regurgitation. Journal of the American College of Cardiology. 1999;33:843-853

29. Lam JH, Ranganathan N, Wigle ED, Silver MD. Morphology of the human mitral valve. I. Chordae tendineae: A new classification. Circulation. 1970;41:449-458

30. Otsuji Y, Handschumacher MD, Schwammenthal E, Jiang L, Song JK, Guerrero JL, Vlahakes GJ, Levine RA. Insights from three-dimensional echocardiography into the mechanism of functional mitral regurgitation: Direct in vivo demonstration of altered leaflet tethering geometry. Circulation. 1997;96:1999-2008

31. Langer F, Schafers HJ. Ring plus string: Papillary muscle repositioning as an adjunctive repair technique for ischemic mitral regurgitation. The Journal of thoracic and cardiovascular surgery. 2007;133:247-249 
32. Veronesi F, Corsi C, Sugeng L, Caiani EG, Weinert L, Mor-Avi V, Cerutti S, Lamberti C, Lang RM. Quantification of mitral apparatus dynamics in functional and ischemic mitral regurgitation using realtime 3-dimensional echocardiography. Journal of the American Society of Echocardiography: official publication of the American Society of Echocardiography. 2008;21:347-354 


\section{Chapter 4}

Left ventricular dyssynchrony is associated with recurrence of ischemic mitral regurgitation after restrictive annuloplasty

Leen Van Garsse, MD, Sandro Gelsomino, MD, PhD, Fabiana Lucà, MD, Orlando Parise, $\mathrm{MS}_{\mathrm{C}}$, Roberto Lorusso MD, PhD , EmileCheriex, MD, PhD, Sabina Caciolli, MD, Enrico Vizzardi, MD, Carmelo Massimiliano Rao, MD, Rocco Carella, MD, Gian Franco Gensini, MD, Jos Maessen, MD, PhD.

Published in: Int J Cardiol. Sept 15, 2012. In press. 


\section{Abstract}

Background: In our study, we investigated the impact of papillary muscle systolic dyssynchrony (DYS-PAP) obtained by 2D speckle-tracking echocardiography (2D-STE) in the prediction of recurrent ischemic mitral regurgitation (MR) after restrictive annuloplasty.

Methods: The study population consisted of 524 consecutive patients who survived coronary artery bypass grafting (CABG) and restrictive annuloplasty, performed between 2001 and 2010 at 3 different Institutions and who met inclusion criteria. The assessment of DYS-PAP was performed preoperatively and at follow-up (median 45.3 months [IQR26-67]) by 2D-STE in the apical four-chamber view for the anterolateral papillary muscle (ALPM) and apical long-axis view for the posteromedial papillary muscle (PMPM).

Results: Recurrence of MR ( $\geq 2+$ in patients with no/trivial MR at discharge) was found in 112 patients $(21.3 \%)$ at follow up. Compared to patients without recurrence of MR, these patients had higher DYS-PAP values at baseline $(60.6 \pm 4.4 \mathrm{~ms}$ vs. $47.2 \pm 2.9 \mathrm{~ms}$, $\mathrm{p}<0.001)$ which significantly worsened at follow up $(74.4 \pm 5.2 \mathrm{~ms}, \mathrm{p}=0.002$ vs. baseline). In contrast, in patients with no MR recurrence, DYS-PAP was significantly reduced $(25.3 \pm 4.4 \mathrm{~ms}, \mathrm{p}=0.002 \mathrm{vs}$. baseline). At logistic regression analysis DYS-PAP (odds ratio [OR]: 4.8, 95\% Confidence Interval [Cl]: $3.4-8.2, p<0.001)$, was the strongest predictor of recurrent $\mathrm{MR}$ with a cutoff $\geq 58 \mathrm{~ms}(95 \% \mathrm{Cl}: 51-66 \mathrm{~ms})$. The model showed an area under the Receiver Operating Characteristic (ROC) curve of 0.97 (Cl 94-99\% [optimismcorrected 94\%; Cl 89-95\%]) with 98\% sensitivity (Cl 96-100\% [optimism-corrected 95\%; $\mathrm{Cl} 91-96 \%$ ]) and 90\% specificity (Cl 85-94\% [optimism-corrected 87\%; Cl 82-90\%]).

Conclusions: DYS-PAP represents a reliable tool to identify patients with ischemic MR who can benefit from restrictive annuloplasty. 


\section{Introduction}

Undersized Mitral Ring Annuloplasty (UMRA) combined with coronary artery bypass grafting $(C A B G)^{1}$ is the conventional approach for the surgical management of patients with moderate to severe chronic ischemic mitral regurgitation (CIMR). Nonetheless, a considerable number of patients show persistent or recurrent mitral regurgitation (MR) despite annuloplasty ${ }^{2,3}$ which adversely affects patient outcomes ${ }^{4}$. Recent studies have confirmed that some patients with CIMR may benefit from UMRA ${ }^{5}$, so the search for preoperative parameters that may help to identify patients who are likely to fail continues.

There are multiple factors that interact in causing CIMR: global left ventricular (LV) negative remodeling ${ }^{6}$, decrease in LV closing forces ${ }^{7}$ and decrease in systolic mitral annulus contraction ${ }^{8}$ which may alter the mitral valve apparatus geometry, thereby causing a lack of leaflet coaptation and inducing functional mitral regurgitation. However, dyssynchrony between myocardial segments adjacent to papillary muscles may play an important role in the mechanisms for $\mathrm{MR}^{9}$ and the reduction in the amount of insufficiency after cardiac resynchronization therapy in patients with LV failure has been associated with the improved coordination of papillary muscles contraction after resynchronization $^{10,11}$.

Two-dimensional speckle-tracking echocardiography (2D-STE) enables the angleindependent assessment of multidirectional LV strain and differentiates myocardial segments with active contraction from segments that are passively tethered ${ }^{12,13}$.

In our study we investigated the impact of papillary muscle systolic dyssynchrony (DYS-PAP) in predicting recurrent MR in patients with CIMR undergoing UMRA.

\section{Materials and methods}

\section{Subjects}

CIMR was defined as the combination of mild-to-severe MR with (1) prior myocardial infarction (MI) >16 days, (2) 75\% or greater stenosis of at least one coronary vessel, (3) a corresponding regional wall motion abnormality, (4) type IIIb leaflet dysfunction following Carpentier's classification ${ }^{14}$ with or without annular dilatation. Exclusion criteria were (1) degenerative or other non-ischemic etiology, (2) ischemic isolated type I or type II dysfunction ${ }^{14}$, (3) additional mitral valve repair procedures, (4) other valvular or congenital heart diseases, (5) previous cardiac surgery, (6) atrial fibrillation, (7) residual $M R$ defined as $M R \geq 2$ at discharge, and (8) echocardiograms not available/incomplete or images not appropriate for 2D-STE analysis.

Among 754 patients with CIMR who survived combined CABG and UMRA performed at three Institutions (Careggi Hospital, Florence, Italy; Civic Hospital, Brescia, 
Italy; University Hospital, Maastricht, The Netherlands) between 2001 and 2010, 524 met inclusion criteria and were included in the study. Fifty age- and gender-matched healthy adults were controls.

Clinical follow-up information was obtained from all survivors through outpatient visits and phone calls and was $100 \%$ complete. Median follow-up was 45.3 months [interquartile range (IQR) 26-67].

Ethical Committee approval was waived due to the retrospective analysis of the study according to National laws regulating observational retrospective studies (Italian law nr.11960, released on 13/7/2004; Dutch WMO law). However, all patients gave their informed consent to access their data for scientific purposes.

The authors had full access to and take full responsibility for the integrity of the data. All authors have read and approved the manuscript as written.

\section{Surgery}

Mitral annuloplasty was performed with standard operative techniques, including cardiopulmonary bypass and undersized annuloplasty ring placement. The ring size was determined by standard measurements of the inter-trigonal distance and anterior leaflet height. Downsizing by two ring sizes was performed in all patients.

All patients underwent associated CABG. For the purposes of this study, complete revascularization was accomplished when at least one graft was placed distal to an approximately $50 \%$ diameter narrowing in each of the three major vascular systems in which arterial narrowing of this severity was noted in a vessel $\geq 1.5 \mathrm{~mm}$ in diameter. It was not considered necessary to bypass all obstructed diagonal branches of the anterior descending or marginal branches of the circumflex coronary arteries for a classification of complete revascularization. Following this definition, $100 \%$ of patients underwent complete revascularization. After cardiopulmonary bypass, a transesophageal echocardiography was performed to assess residual MR: leaflet coaptation $\geq 0.8 \mathrm{~cm}$, $M R \leq 1$, and systolic $M V$ area $>2 \mathrm{~cm}^{2}$ was assessed as successful repair.

\section{Echocardiography}

Transthoracic echocardiography was performed within a week before surgery and at follow-up appointments. Exams were carried out using a commercially available ultrasound system (IE 33, Philips Medical System, Amsterdam, The Netherlands). Images were acquired by three experienced sonographers (E.C., S.C., E.V.), stored in DICOM format and transferred to a workstation for further offline analysis (TOMTEC Imaging system, Unterschleißheim, Germany). Measurements and calculations were made separately by one of the investigators (F.L.). The reliability of echocardiographic measurements was assessed by calculating intra-observer intervals of agreement of main direct measures used in this study in 20 subjects randomly chosen among the study 
patients. The Bland-Altman method (Table 1) showed excellent agreement between intra-observer measurements in both low and high values of echocardiographic parameters.

Table 1. Bland -Altman limits of agreement for intra-observer variability.

\begin{tabular}{lccc}
\hline & Bias & Standard Deviation & 95\% Limit of Agreement \\
\hline ALPM-LS & 0.01 & 0.18 & -0.34 to 0.38 \\
PMPM-LS & -0.05 & 0.20 & -0.44 to0.32 \\
GLS $_{\text {peak }}$ & -0.02 & 0.41 & -0.48 to 0.42 \\
DYS-PAP & -0.01 & 0.33 & -0.63 to 0.56 \\
\hline
\end{tabular}

Intra-observer and inter-observer relative differences were $<5 \%$ for all parameters. The Bland-Altman method showed excellent agreement between intra-observer measurements in both low and high values of echocardiographic parameters. Abbreviations. ALPM-LS = Anterolateral papillary muscle longitudinal strain ; PMPM-LS = Posteromedial papillary muscle longitudinal strain; GLSpeak = Peak Global longitudinal strain; DYS-PAP = Papillary muscle dyssynchrony.

\section{Assessment of MR and LV geometry}

The following quantitative measurements were simultaneously used to grade the severity of MR: (1) Pulsed Doppler quantitative flow methods and (2) proximal isovelocity surface area (PISA). When the evidence from different parameters was congruent, the measurements were averaged, allowing the calculation of effective regurgitant orifice area (EROA), regurgitant volume (RV) and regurgitant fraction $(\mathrm{RF})^{15}$.

When different parameters were contradictory, PISA was chosen in case of a central jet or in the presence of a calcific mitral valve or mitral annulus, whereas pulsedwave Doppler was preferred when the jet was eccentric or multiple ${ }^{15}$. For each measurement, a minimum of three cardiac cycles were averaged. In patients with no or trivial MR by color Doppler, RV and RF were used as calculated, and EROA was assumed as null. The respective thresholds for mild (2+), moderate (3+) and severe (4+) MR were $<30,30-59$ and $\geq 60 \mathrm{~mL} /$ beat for RV; $<30,30-49$, and $\geq 50 \%$ for RF; $<20,20-39$, and $\geq 40 \mathrm{~mm}^{2}$ for EROA. Recurrent MR at latest follow-up was defined as insufficiency $\geq 2+$ in patients with no or trivial MR at discharge.

Left ventricular volumes and LV ejection fraction (LVEF) were assessed using the biapical Simpson disk method ${ }^{16}$. Sphericity indexes were obtained at end-diastole and end-systole as the volume of the LV divided by the volume of a sphere with a diameter equal to the longest axis of the LV measured in the apical view ${ }^{17}$. The wall motion score index (WMSI) was calculated according to a 17-segment model as previously reported ${ }^{3}$.

\section{Mitral valve configuration}

Mitral valve configuration was assessed in mid-systole using the parasternal long-axis and four-chamber views. The tenting area (TA) was measured by the area enclosed 
between the annular plane and the mitral leaflets from the parasternal long-axis view at mid-systole. The coaptation height $(\mathrm{CH})$ was measured as the perpendicular distance between the coaptation point of the mitral leaflets and the line connecting the annular hinge points in the long-axis view at end-systole. The coaptation length (CL) was measured as the length of apposition of the anterior mitral leaflet (AML) and posterior mitral leaflet (PML). The coaptation distance (CD) was measured (along the annular plane) from the AML attachment to the point of coaptation. Mitral annular areas were obtained from mitral annular dimensions in apical long-axis, four-chamber, and two-chamber views, using an ellipsoid assumption ${ }^{18}$. The AML tethering angle $\alpha$ (between the annular plane and the basal part of $A M L)$ and the PML tethering angle $\beta$ (between the annular plane and the basal part of PML) were directly measured with specific software (Philips DICOM Viewer; Philips Medical System). The ratio of $\alpha$ to $\beta$ was a quantitative measurement of tethering: the closer the ratio was to 1 , the more symmetric was the tethering ${ }^{19}$.

\section{Two-dimensional speckle-tracking echocardiography}

The assessment of longitudinal peak systolic strain was performed by applying 2D-STE to the apical two- and four-chamber views of the LV. The LV was divided into six segments in each apical view and six global longitudinal strain (GLS) curves plus an average GLS curve were obtained from each view. With the beginning of the QRS complex as reference point, the peak longitudinal strain was the peak negative values on GLS curves during the ejection phase. The peak global longitudinal strain $\left(G L S_{\text {peak }}\right)$ was the mean of the two strain values obtained from the average global longitudinal strain curves in the two views.

The assessment of papillary muscles was performed by applying 2D-STE to the apical four-chamber view for anterolateral papillary muscle (ALPM) and to the apical longaxis view for posteromedial papillary muscle (PMPM) ${ }^{9}$.

From an end-systolic single frame, each papillary muscle was traced on the endocardial cavity interface by a point-and-click approach from the tip to the base. Following the papillary muscle tracing, an automated tracking algorithm (free-trace method, Tomtec, Tomtec Imaging system, Unterschleißheim, Germany) followed the muscle from this single frame throughout the cardiac cycle. Further adjustment of the region of interest was performed to ensure that all of the papillary muscle was included ${ }^{9}$.

The average value of peak systolic longitudinal strain for each papillary muscle was then determined as anterolateral papillary muscle longitudinal strain (ALPM-LS) and posteromedial papillary muscle longitudinal strain (PMPM-LS). The beginning of the QRS complex was used as the reference point and the time to peak (TTP) systolic longitudinal strain was quantified for each papillary muscle. For the assessment of papillary muscle systolic dyssynchrony (DYS-PAP), the absolute difference in TTP between ALPM and PMPM, was calculated. 


\section{Statistical analysis}

Variables were expressed as mean $\pm S D$, median and interquartile range, or number of patients (percentage) as appropriate. For within-group comparisons before and after annuloplasty, paired t test, Wilcoxon or Mac Nemar tests were used. Between-group differences were assessed by unpaired t test, Mann-Whitney test, or $\chi^{2}$ test. ANOVA and Tukey's post-hoc test were employed to test whether there was any difference in DYS-PAP values with respect to the myocardial infarction site.

Linear regression analysis was employed to study the relationship of DYS-PAP with QRS width, baseline WMSI and postoperative regurgitant volume.

Multivariable logistic regression with the forward stepwise method was performed to identify preoperative echocardiographic predictors of recurrent MR. The predictors tested were end diastolic diameter [EDD], end systolic diameter [ESD], end diastolic volume [EDV], end systolic volume [ESV], LVEF, systolic sphericity Index [SI $\mathrm{Sys}_{\mathrm{sy}}$, wall motion score index $\left[\mathrm{WMSI}\right.$, diastolic sphericity Index $\left[\mathrm{SI}_{\mathrm{Dia}}\right], \mathrm{TA}, \mathrm{CH}, \mathrm{CL}, \mathrm{CD}, \mathrm{AML}$ tethering angle $\alpha$, PML tethering angle $\beta, \alpha / \beta$ ratio, DYS-PAP, ALPM-LS, PMPM-LS and G$\mathrm{LS}_{\text {peak }}$. The model was reliable (Hosmer-Lemeshow test 3.26; $\mathrm{p}=.87$ ) and accurate, with a c-index of 0.85 (95\% confidence interval [CI] 0.82-0.87).

Optimal cut-off values were determined by receiver operating characteristic (ROC) curve as the optimal threshold for predicting recurrence of MR. We validated results using the bootstrap method (1000 iterations) and computed the optimism-corrected estimate ("optimism" refers to absolute magnitude of bias) for sensitivity, specificity and area under the ROC curve.

To test whether the predictive value of DYS-PAP was independent of LV volumes and other predictors of recurrent $\mathrm{MR}^{3,19}$, we tested for interactions by entering interaction terms between DYS-PAP and other parameters with an interaction $p<0.10$ considered statistically significant.

Statistical analysis was performed with SAS, release 9.2 ( SAS Institute, Cary, NC). ROC curve optimism analysis was conducted with S-plus statistical software, release 6.0 (Insightful Corp, Seattle, WA).

The authors of this manuscript have certified that they comply with the Principles of Ethical Publishing in the International Journal of Cardiology ${ }^{20}$.

\section{Results}

\section{Recurrence of mitral regurgitation}

Recurrent MR was found in 112 (21.3\%) patients at follow-up (MR+ Group) whereas in $412(78.7 \%)$ it was absent or trivial (MR- Group). There was no difference in the incidence of recurrent mitral regurgitation between the first (2001-2005) and the last 
years (2006-2008): $20.9 \%$ [45/215] vs.: $21.6 \%$ [67/309], $p=0.84$. Furthermore, no difference was found in baseline characteristics between the 2 groups (Table 2). At follow-up the MR+ group had higher New York Heart Association class $(p<0.001)$ and showed a trend toward higher MR grade $(p<0.001)$, compared with the MR-Group.

\section{Changes in LV geometry and mitral valve configuration after annuloplasty}

Patients in the MR+ Group had at baseline larger LV volumes and a more spherical ventricle (Table 3). At follow-up, in patients in the MR-Group, ESV decreased significantly $(p<0.001)$ while in the MR+ Group it increased compared to baseline values $(p<0.001)$. Changes in EDV showed the same tendency as ESV. Systolic and diastolic sphericity indexes reduced significantly in the MR- Group $(p<0.001)$, whereas both indexes increased in patients with recurrent $M R(p=0.01$ and $p=0.04$, respectively). Furthermore, WMSI was reduced significantly in MR- patients $(p=0.001)$ while it did not change in the MR+ Group ( $p=0.77)$.

There was no significant difference between the two groups regarding quantitative baseline MR data. Baseline TA $(p=0.01)$ and $\mathrm{CH}(p<0.001)$ were higher whereas baseline $C L$ was lower $(p=0.001)$ in patients with recurrent MR. Contrastingly, the CD was comparable between groups $(p=0.5)$. Additionally, the anterior tethering angle $\alpha$ $(p<0.002)$ was larger in the MR+ Group whereas the posterior tethering angle $\beta$ did not differ between groups $(p=0.6)$. Finally, patients with recurrent MR had a more symmetrical tethering $(p<0.001)$ at baseline.

At follow-up, in the MR-Group, TA $(p<0.001)$ and $\mathrm{CH}(\mathrm{p}=0.03)$ decreased while $\mathrm{CL}$ $(<0.001)$ increased significantly. These indices did not change in the MR+Group. Furthermore, systolic and diastolic mitral annular areas decreased significantly $(p<0.001)$ in both Groups compared to their baseline values, but without significant differences between both Groups.

The $\beta$ angle increased significantly in both Groups whereas $\alpha$ decreased only in the MR-Group. When the degree of tethering was examined at follow-up it became progressively more asymmetrical in both Groups ( $p<0.001$ vs. baseline). 
Table 2. Clinical Characteristics $(n=524)$

\begin{tabular}{|c|c|c|c|}
\hline & $\begin{array}{c}\text { MR -Group } \\
(n=412)\end{array}$ & $\begin{array}{c}\text { MR + Group } \\
(n=112)\end{array}$ & $\mathrm{p}$ \\
\hline Age, $y$ & $68.2 \pm 7.0$ & $67.4 \pm 6.8$ & 0.7 \\
\hline Gender M/F & $247 / 165(59.9 / 40.1)$ & $61 / 51(54.4 / 45.5)$ & 0.3 \\
\hline NYHA class & $3[3-4]$ & $3[3-4]$ & $>0.9$ \\
\hline CCS angina class & $2[1-3]$ & $2[1-3]$ & $>0.9$ \\
\hline \multicolumn{4}{|l|}{ Euroscore } \\
\hline Additive & $8.5[6.6-11.8]$ & $9.3[7.1-12.4]$ & 0.1 \\
\hline Logistic & $14.4[10.2-16.9]$ & $15.3[11.0-17.6]$ & 0.08 \\
\hline Hypertension & $164(39.8)$ & $44(39.2)$ & 0.9 \\
\hline Diabetes & 107 (25.9) & $25(22.3)$ & 0.1 \\
\hline COPD & $61(14.8)$ & $15(13.3)$ & 0.5 \\
\hline Chronic renal disease & $66(16.0)$ & $18(16.0)$ & $>0.9$ \\
\hline Cerebral vascular disease & 45 (10.9) & $9(8.0)$ & 0.09 \\
\hline Peripheral vascular disease & $33(8.0)$ & $13(11.6)$ & 0.09 \\
\hline Family history & $164(39.8)$ & $49(43.7)$ & 0.1 \\
\hline IABP & $28(6.7)$ & $11(9.8)$ & 0.07 \\
\hline \multicolumn{4}{|l|}{ Myocardial infarction } \\
\hline Inferior/Posterior & 173 (41.9) & $44(39.3)$ & \\
\hline Anterior/Septal & $19(4.7)$ & $5(4.5)$ & 0.6 \\
\hline Lateral & $27(6.6)$ & $8(7.1)$ & \\
\hline Combined & $193(46.8)$ & 55 (49.1) & \\
\hline Coronary vessel disease & $2[2-3]$ & $2[2-3]$ & $>0.9$ \\
\hline Left Main & $94(22.8)$ & $24(21.4)$ & 0.9 \\
\hline \multicolumn{4}{|l|}{ Medications } \\
\hline ACEI/ARB & $375(91.0)$ & $105(93.7)$ & \\
\hline B-adrenergic blockers & $210(50.9)$ & $58(51.7)$ & \\
\hline Long-acting nitrates & $173(42.0)$ & $49(43.7)$ & 0.44 \\
\hline Diuretics & 350 (84.9) & $97(86.6)$ & \\
\hline Spironolactone & 222 (53.9) & $63(56.2)$ & \\
\hline \multicolumn{4}{|l|}{ Surgery } \\
\hline CPB time (min) & $101[80-115]$ & $107[88-120]$ & 0.07 \\
\hline $\mathrm{CCL}$ time (min) & $80[66-98]$ & 87 [70-109] & 0.1 \\
\hline Mitral Ring size (mm) & $28[26-28]$ & $28[26-28]$ & $>0.9$ \\
\hline \multicolumn{4}{|l|}{ Follow-up } \\
\hline NYHA class & $1[1-2]$ & $2[2-3]$ & $<0.001$ \\
\hline NYHA III/IV & $126(30.5)$ & $85(75.8)$ & $<0.001$ \\
\hline Reappearance of Angina & $15(3.7)$ & $9(8.7)$ & 0.06 \\
\hline ACS & $1(0.2)$ & $2(1.9)$ & 0.12 \\
\hline \multicolumn{4}{|l|}{ Coronary Procedures } \\
\hline Redo CABG & $1(0.2)$ & $0(0)$ & 0.78 \\
\hline $\mathrm{PCl}$ & $13(3.1)$ & $6(5.3)$ & 0.28 \\
\hline
\end{tabular}

Normally- distributed variables are presented as mean \pm standard deviation; Discrete variables are presented as percentages. Non-normally distributed variables are presented as median [Interquartile range].

Abbreviations: $\mathrm{M} / \mathrm{F}=$ Male/Female; NYHA = New York Heart Association; $\mathrm{CCS}=$ Canadian Cardiovascular Society; COPD = Chronic obstructive pulmonary disease; IABP = Intra-aortic balloon pump; $\mathrm{ACEI}=$ angiotensin-converting enzyme inhibitor; $\mathrm{ARB}=$ angiotensin receptor blocker. $\mathrm{CPB}=$ Cardiopulmonary bypass; $\mathrm{CCL}=$ (Aortic) Cross-clamp; $\mathrm{CABG}=$ Coronary artery bypass grafting. $\mathrm{ACS}=$ Acute coronary syndrome; $\mathrm{PCl}=\mathrm{Percu}-$ taneous coronary intervention. 
Table 3. LV Geometry and Mitral Valve Configuration

\begin{tabular}{|c|c|c|c|c|c|c|c|}
\hline & \multirow[b]{2}{*}{ Controls } & \multicolumn{3}{|c|}{$M R-G r o u p(n=412)$} & \multicolumn{3}{|c|}{$M R+$ Group $(n=112)$} \\
\hline & & Preoperative & Follow up & $\mathrm{p}$ & Preoperative & Follow up & $\mathrm{p}$ \\
\hline LVEF (\%) & $59 \pm 8$ & $34 \pm 11^{\ddagger}$ & $38 \pm 10^{\ddagger}$ & 0.71 & $29 \pm 9^{\neq}$ & $37 \pm 9$ & 0.04 \\
\hline ESV $(m L)$ & $40 \pm 12$ & $133 \pm 20^{\ddagger}$ & $107 \pm 16^{\ddagger}$ & $<0.001$ & $162 \pm 21^{*}$ & $173 \pm 26^{\ddagger \neq}$ & $<0.001$ \\
\hline $\mathrm{EDV}(\mathrm{mL})$ & $97 \pm 18$ & $195 \pm 32^{\ddagger}$ & $135 \pm 26^{\ddagger}$ & $<0.001$ & $211 \pm 38^{*}$ & $230 \pm 39^{\dagger \ddagger}$ & $<0.001$ \\
\hline $\mathrm{SI}_{\text {Sys }}$ & $0.52 \pm 0.1$ & $0.67 \pm 0.1^{\ddagger}$ & $0.54 \pm 0.1$ & $<0.001$ & $0.75 \pm 0.1^{*}$ & $0.82 \pm 0.1^{\ddagger \neq}$ & 0.01 \\
\hline $\mathrm{SI}_{\text {Dia }}$ & $0.64 \pm 0.1$ & $0.74 \pm 0.1^{\ddagger}$ & $0.64 \pm 0.1$ & $<0.001$ & $0.83 \pm 0.1^{*}$ & $0.89 \pm 0.1^{\dagger \neq}$ & 0.04 \\
\hline WMSI & $1.0 \pm 0.1$ & $1.3 \pm 0.3^{\ddagger}$ & $1.1 \pm 0.2$ & 0.001 & $1.6 \pm 0.3^{*} \ddagger$ & $1.5 \pm 0.3^{\dagger \ddagger}$ & 0.77 \\
\hline MR & - & $3[3-4]$ & $1[1-2]$ & $<0.001$ & $3[3-4]$ & $3[3-4]$ & $>0.9$ \\
\hline $\operatorname{EROA}\left(\mathrm{mm}^{2}\right)$ & - & $36.9 \pm 11.2$ & - & - & $37.4 \pm 10.7$ & $32.6 \pm 10.3^{\dagger}$ & 0.07 \\
\hline $\mathrm{RF}(\%)$ & - & $45.2 \pm 10.6$ & - & - & $47.1 \pm 11.4$ & $44.1 \pm 13.6^{\dagger}$ & 0.1 \\
\hline $\mathrm{RV}$ (ml/beat) & - & $57.9 \pm 10.8$ & - & - & $58.1 \pm 10.6$ & $55.0 \pm 15.4^{\dagger}$ & 0.1 \\
\hline $\mathrm{TA}\left(\mathrm{cm}^{2}\right)$ & $0.8 \pm 2$ & $3.0 \pm 1.1^{\ddagger}$ & $1.9 \pm 0.3^{\ddagger}$ & $<0.001$ & $3.9 \pm 1.1^{* \neq}$ & $3.4 \pm 1.3^{\dagger \ddagger}$ & 0.06 \\
\hline $\mathrm{CL}(\mathrm{mm})$ & $8.4 \pm 0.4$ & $4.2 \pm 0.5^{\ddagger}$ & $8.9 \pm 0.4$ & $<0.001$ & $3.2 \pm 0.3^{*}$ & $3.3 \pm 0.3^{\dagger \neq}$ & 0.8 \\
\hline $\mathrm{CD}(\mathrm{mm})$ & $26.9 \pm 6.2$ & $35.9 \pm 7.1^{\ddagger}$ & $31.6 \pm 5.5^{\ddagger}$ & 0.62 & $36.6 \pm 5.0^{\ddagger}$ & $39.1 \pm 8.4^{\dagger \neq}$ & 0.6 \\
\hline $\mathrm{CH}(\mathrm{mm})$ & $6.0 \pm 2.3$ & $9.9 \pm 1.9^{\ddagger}$ & $6.8 \pm 0.8$ & 0.03 & $13.2 \pm 2.2^{* \ddagger}$ & $12.4 \pm 1.5^{\dagger \neq}$ & 0.8 \\
\hline $\mathrm{MA}_{\mathrm{S}}\left(\mathrm{cm}^{2}\right)$ & $4.5 \pm 0.5$ & $4.1 \pm 1.2$ & $2.6 \pm 0.7^{\ddagger}$ & $<0.001$ & $4.3 \pm 1.3$ & $2.5 \pm 0.6^{\ddagger}$ & $<0.001$ \\
\hline $\mathrm{MA}_{\mathrm{d}}\left(\mathrm{cm}^{2}\right)$ & $7.1 \pm 0.7$ & $5.0 \pm 1.3^{\ddagger}$ & $3.4 \pm 0.9^{\ddagger}$ & $<0.001$ & $5.1 \pm 1.2^{\ddagger}$ & $3.7 \pm 0.8^{\ddagger}$ & $<0.001$ \\
\hline \multicolumn{8}{|c|}{ Mitral Leaflet Tethering } \\
\hline$\beta^{\prime}\left(^{\circ}\right)$ & $23.2 \pm 3.1$ & $53.2 \pm 10.1^{\ddagger}$ & $73.7 \pm 13.9^{\ddagger}$ & $<0.001$ & $51.7 \pm 9.6^{\ddagger}$ & $78.1 \pm 12.0^{\ddagger}$ & $<0.001$ \\
\hline$\alpha\left(^{\circ}\right)$ & $34.1 \pm 5.8$ & $35.8 \pm 4.1^{\ddagger}$ & $29.5 \pm 3.3^{\ddagger}$ & $<0.006$ & $43.4 \pm 6.9 *^{\ddagger}$ & $37.0 \pm 5.6^{\dagger \neq}$ & 0.06 \\
\hline$\alpha / \beta$ & $0.72 \pm 0.2$ & $0.66 \pm 0.1^{\ddagger}$ & $0.43 \pm 0.1^{\ddagger}$ & $<0.001$ & $0.81 \pm 0.1 *^{\dagger \ddagger}$ & $0.49 \pm 0.2^{\ddagger}$ & $<0.001$ \\
\hline
\end{tabular}

Continuous variables are presented as mean \pm standard deviation; Non-normally distributed variables are presented as median [Interquartile range]. p: significance at paired t test; ${ }^{*}$ Significance vs. MR- at baseline at unpaired t test; + significance vs. MR- at follow-up at unpaired $t$ test. ${ }^{\ddagger}$ significance vs. controls.

Abbreviations: $\mathrm{LVEF}=$ Left Ventricular Ejection Fraction; ESV = End-systolic Volume; EDV = End-diastolic Volume; $\mathrm{MR}=$ Mitral regurgitation; $\mathrm{SI}_{\text {Sys }}=$ Systolic Sphericity Index; $\mathrm{SI}_{\mathrm{Dia}}=$ Diastolic Sphericity Index; WMSI = Wall Motion Score Index; EROA = Effective Regurgitant Orifice Area; RF = Regurgitant Fraction; RV = Regurgitant Volume; $\mathrm{TA}=$ Tenting Area; $\mathrm{CL}=$ Coaptation length; $\mathrm{CD}=$ Coaptation Distance; $\mathrm{CH}=$ Coaptation Height; MAs = Systolic Mitral Annular Area; $\mathrm{MA}_{d}=$ Diastolic Mitral Annular Area; $\beta=$ posterior mitral leaflet tethering angle; $\alpha=$ anterior mitral leaflet tethering angle; $\alpha / \beta=$ tethering pattern.

\section{Two-dimensional speckle-tracking analysis}

Speckle tracking analysis is shown in Table 4. Patients in the MR+ Group had higher baseline DYS-PAP ( $p<0.001$; [Figure 1]) and worse values of ALPM-LS ( $p<0.001)$, PMPMLS ( $p<0.001)$ and global longitudinal strain peak ( $p<0.00$; [Figure 2]). At follow-up control, in patients with MR recurrence, ALPM-LS ( $p=0.8)$ PMPM-LS $(p=0.8)$ and $G L S_{\text {peak }}$ $(p>0.9)$ did not change whereas DYS-PAP significantly worsened $(p=0.002)$. In contrast, in patients with no MR recurrence, ALPM-LS ( $p=0.005)$, PMPM-LS ( $p<0.001)$, $\mathrm{GLS}_{\text {peak }}(p<0.001)$ and DYS-PAP $(p=0.002)$ significantly improved (Table 4, Figure 1, Figure 2). No difference was detected in DYS-PAP values (Figure $3 \mathrm{~A}-\mathrm{B}$ ) with respect to 
the infarction site at baseline $(p=0.17)$ as well as at follow up $(p=0.79)$. Furthermore, there was a weak correlation between DYS-PAP and QRS width $(R=0.07, p=0.10)$. In contrast, DYS-PAP strongly correlated with WMSI $(R=0.90, p<0.001)$ and postoperative regurgitant volume $(R=0.92, p<0.001)$.

Table 4. Speckle-tracking Analysis

\begin{tabular}{lccccccc}
\hline & & \multicolumn{3}{c}{ MR -Group $(\mathrm{n}=412)$} & \multicolumn{3}{c}{ MR + Group $(\mathrm{n}=112)$} \\
\cline { 3 - 8 } & Controls & Baseline & Follow-up & $\mathrm{p}$ & Baseline & Follow-up & $\mathrm{p}$ \\
\hline ALPM-LS (\%) & $-22.3 \pm 7.1$ & $-12.7 \pm 0.4^{\ddagger}$ & $-17.7 \pm 0.7^{\ddagger}$ & 0.005 & $-6.6 \pm 0.3^{* \ddagger}$ & $-6.4 \pm 0.2^{\dagger \ddagger}$ & 0.8 \\
PMPM-LS (\%) & $-19.4 \pm 5.9$ & $-11.2 \pm 0.3^{\ddagger}$ & $-14.4 \pm 0.7^{\ddagger}$ & $<0.001$ & $-5.7 \pm 0.3^{* \ddagger}$ & $-5.2 \pm 0.3^{\dagger \ddagger}$ & 0.8 \\
GLS (\%) & $-20.7 \pm 7.0$ & $-10.4 \pm 0.2^{\ddagger}$ & $-15.6 \pm 0.6^{\ddagger}$ & $<0.001$ & $-6.0 \pm 0.2^{* \ddagger}$ & $-6.1 \pm 0.2^{\dagger \ddagger}$ & $>0.9$ \\
DYS-PAP (ms) & $4.8 \pm 2.1$ & $47.2 \pm 2.9^{\ddagger}$ & $25.3 \pm 4.4^{\ddagger}$ & 0.002 & $60.6 \pm 4.0^{* \ddagger}$ & $74.4 \pm 5.2^{\dagger \ddagger}$ & 0.002 \\
\hline
\end{tabular}

p: significance at paired t test; ${ }^{*}$ Significance vs. MR- at baseline at unpaired t test; ${ }^{\dagger}$ significance vs. MR- at follow-up at unpaired t test; ${ }^{\ddagger}$ significance vs. controls.

Abbreviations: ALPM-LS = Anterolateral papillary muscle longitudinal strain; PMPM-LS = Posteromedial papillary muscle longitudinal strain; GLS = Global longitudinal strain; DYS-PAP = Papillary muscle dyssynchrony.
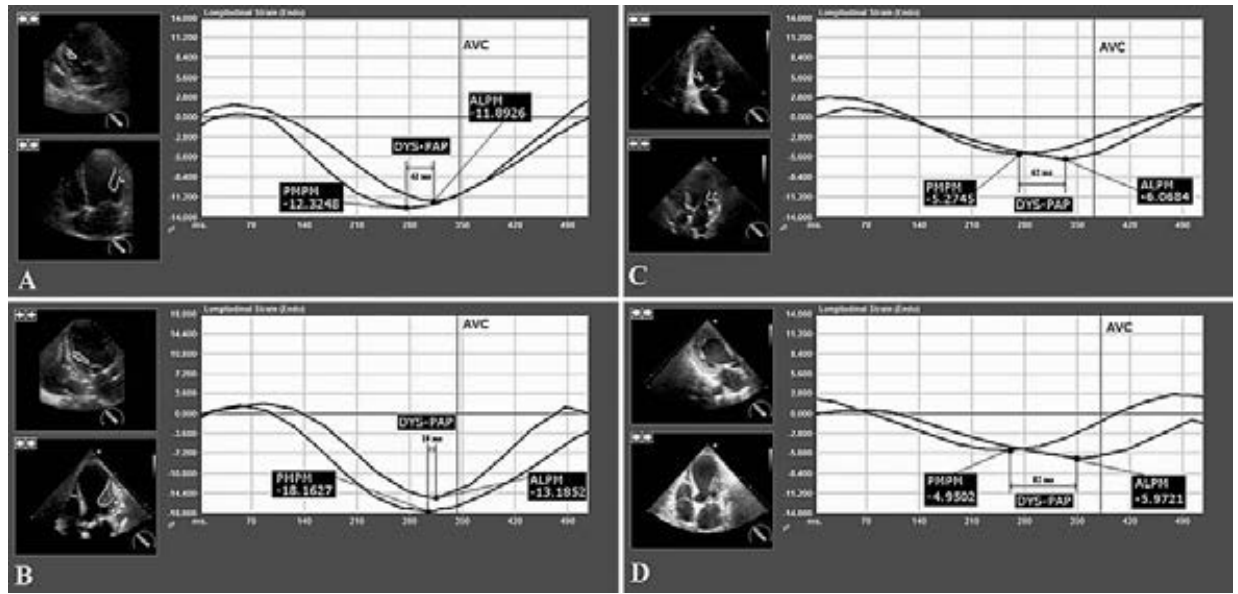

Figure 1. Papillary muscle dyssynchrony (DYS-PAP). A) Preoperative DYS-PAP in a patient without recurrent MR. B) Postoperative DYS-PAP in the same patient as A. C) Preoperative DYSPAP in a patient with MR+. D) Postoperative DYS-PAP in the same patient as $C$.

Abbreviations: AVC: Aortic valve closure; ALPM: Anterolateral papillary muscle; PMPM: posteromedial papillary muscle; DYS-PAP: papillary muscle dyssynchrony. 

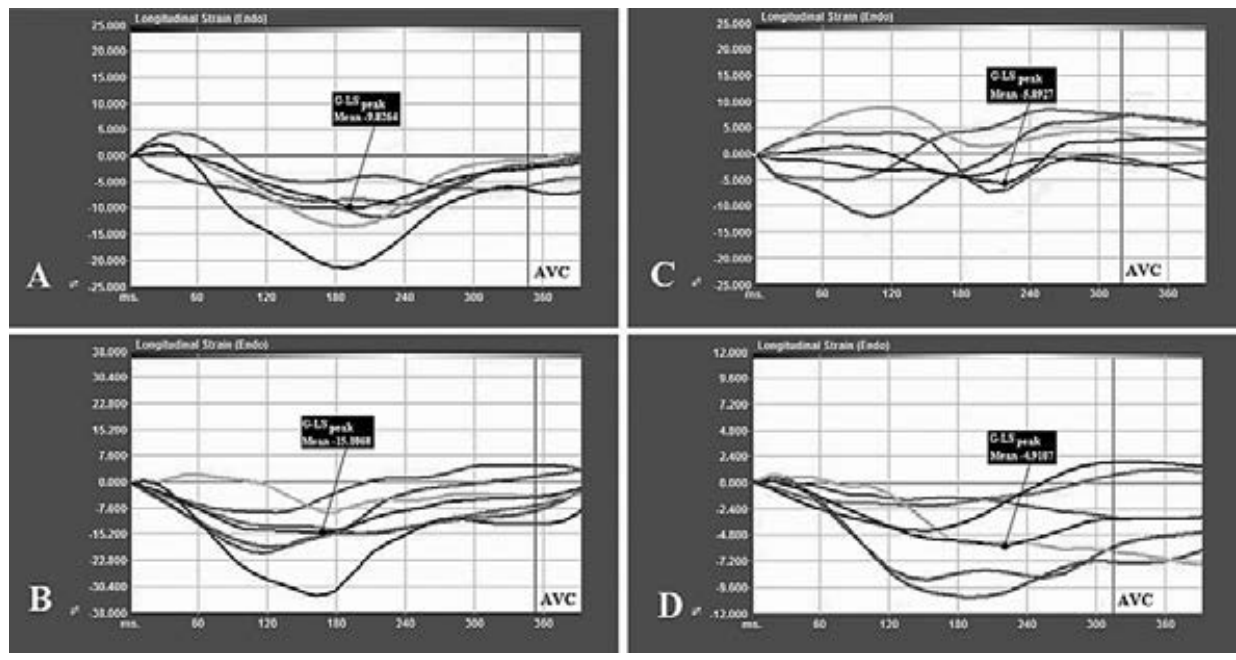

Figure 2. Global longitudinal strain (GLS) curves and global longitudinal strain peak $\left(G L S_{\text {peak }}\right)$ from apical 4chamber view. A) Preoperative GLS curves and $G_{L} S_{\text {peak }}$ in a patient without recurrent MR. B) Postoperative GLS curves and $\mathrm{GLS}_{\text {peak }}$ in the same patient as A. C) Preoperative GLS curves and $\mathrm{GLS}_{\text {peak }}$ in a patient with recurrent MR. D) Postoperative GLS curves and $G_{L} S_{\text {peak }}$ in the same patient as $C$.

Abbreviations: AVC: Aortic valve closure. 
DYS-PAP Baseline

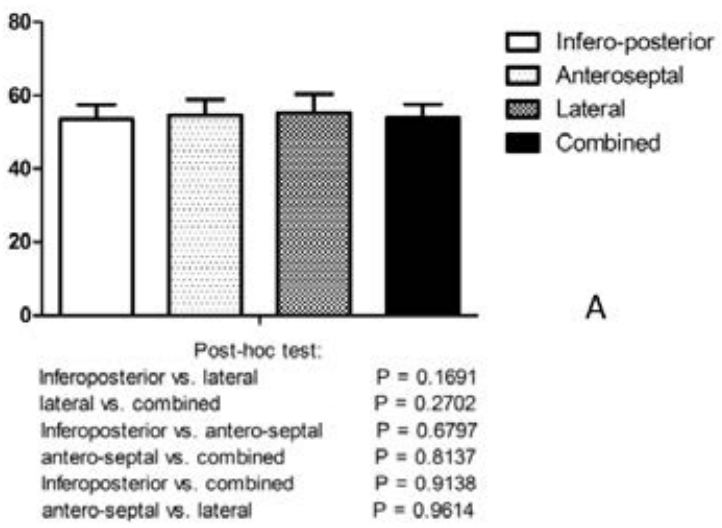

DYS-PAP Follow up

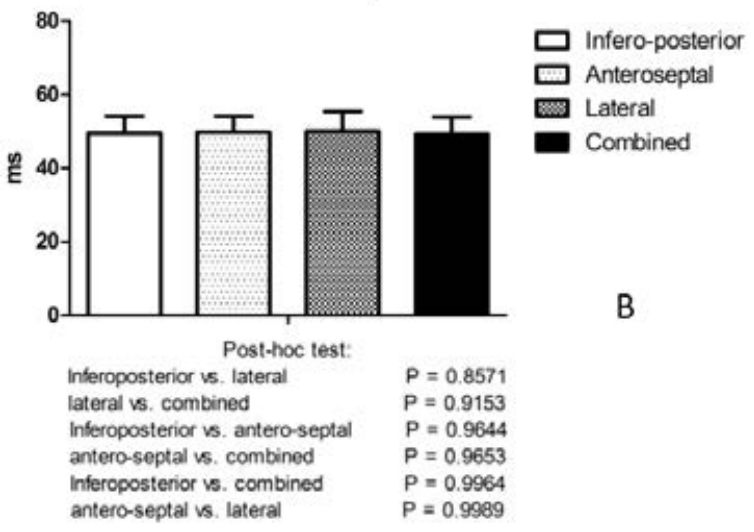

Figure 3. Papillary muscle dyssynchrony (DYS-PAP) values by infarction site at baseline $(A)$ and follow up (B)

\section{Predictors of recurrent MR}

At multivariable analysis (Figure 4) DYS-PAP (odds ratio [OR]: 4.8, 95\% Cl: 3.4-7.2), AML tethering angle $\alpha$ (OR: $4.5,95 \% \mathrm{Cl}: 3.0-7.0)$, tethering symmetry (OR: 3.7, 95\% Cl: $2.3-$ 5.5 ) and systolic sphericity Index (OR: 3.1, 95\% Cl: 2.2-4.7) were predictors of recurrent MR.

The ROC curve analysis (Figure 5A) showed that a cut-off value of DYS-PAP of $\geq 58$ ms was predictive of recurrent MR. Furthermore, optimal cut-off values of the anterior tethering angle, index of tethering symmetry $\alpha / \beta$ and $\mathrm{SI}_{\text {sys }}$ determined by ROC curve analysis were $\geq 39^{\circ}, \geq 0.76$ and $\geq 0.72$, respectively (Figure 5 B-D).

Finally, there were no apparent significant interactions between DYS-PAP and $\alpha$ (interaction $p$ value $=0.15$ ), $\alpha / \beta$ (interaction $p$ value $=0.27$ ), ESV (interaction $p$ val$\mathrm{ue}=0.55$ ) and $\mathrm{SI}_{\mathrm{SYS}}$ (interaction $\mathrm{p}$ value=0.33). 


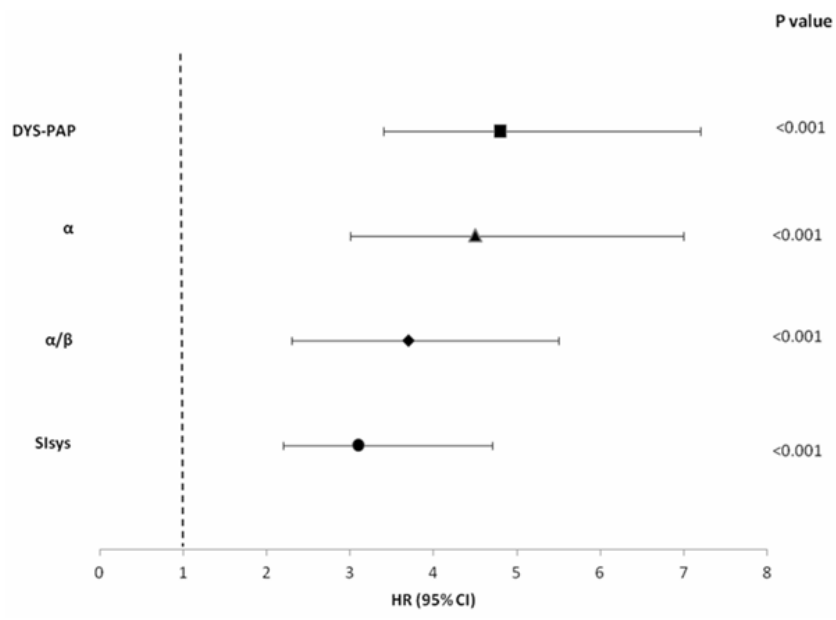

Figure 4. Multivariable predictors of postoperative papillary muscle dyssynchrony.

Abbreviations: DYS-PAP: papillary muscle dyssynchrony; $\alpha$ : anterior mitral leaflet tethering angle; $\beta$ : posterior mitral leaflet tethering angle; SIsys: Systolic sphericity Index.
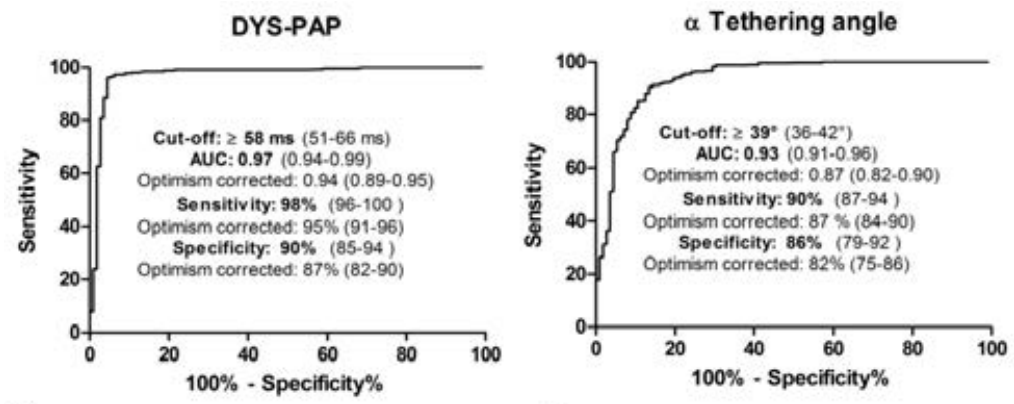

\section{A}

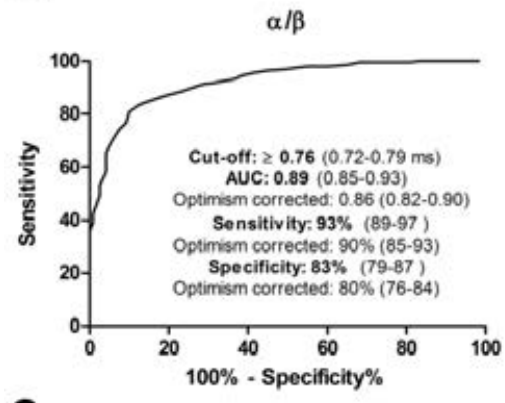

C
B

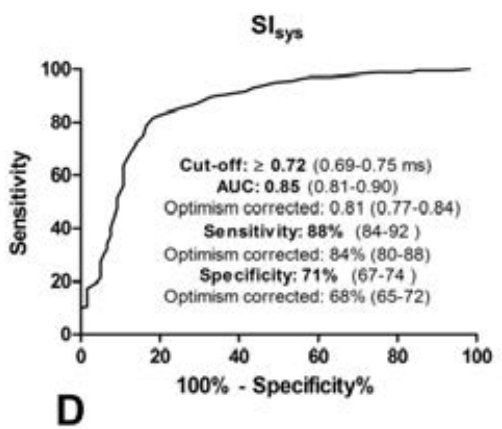

Figure 5. Predictors of recurrent $\mathrm{MR}$. All values are given with $95 \% \mathrm{Cl}$ (brackets). A) The figure shows a receiver operating characteristics (ROC) curve of papillary muscle dyssynchrony (DYS-PAP) in predicting recurrence of mitral regurgitation after restrictive annuloplasty. B) ROC curve of $\alpha$ tethering angle in predicting recurrent MR. C) ROC curve of $\alpha / \beta$ ratio in predicting recurrent MR. D) ROC curve of systolic sphericity Index $\left(\mathrm{SI}_{\mathrm{sys}}\right)$ in predicting recurrent $\mathrm{MR}$. 


\section{Discussion}

\section{Dyssynchrony and functional mitral regurgitation}

Functional mitral regurgitation is a common finding in patients with ischemic mitral disease as a complication of LV dysfunction and LV remodeling ${ }^{6,7}$. Although mitral valve tenting is the main determinant of functional mitral regurgitation, clinical and experimental observations suggest that the dyssynchronic contractions of LV segments in these patients could be a potential co-determinant of functional insufficiency.

Mechanical dyssynchrony of the LV can contribute to functional mitral regurgitation through several different mechanisms.

First, the mechanical dyssynchrony between LV segments supporting the papillary muscles produces uncoordinated regional LV mechanical activation in these segments, resulting in geometrical changes in mitral leaflet attachments and implying tethering of the mitral leaflets ${ }^{21-24}$. Second, a positive pressure gradient develops between the left atrium and left ventricle due to improper timing of atrio-ventricular relaxation and contraction cycles, which can create diastolic FMR during incomplete mitral valve closure $^{25}$. Finally, LV dyssynchrony decreases LV contraction efficiency and closing forces, thereby impairing mitral valve tenting ${ }^{26}$.

\section{Two-dimensional speckle-tracking echocardiography}

Tissue Doppler Imaging (TDI)-derived strain and strain rate imaging were introduced several years ago as a method to quantify myocardial mechanical function and it has been shown to be superior to myocardial velocities by TDI and wall motion scores in assessment of ischemia in experimental and clinical studies ${ }^{27-30}$. Nonetheless, TDIbased strain measurements are angle dependent owing to the use of the Doppler effect and simultaneous opposite deformation in the long and short axes ${ }^{27,28,31}$. Furthermore, this method encountered a number of criticisms, particularly in relation to noise interference, and substantial intra-observer and inter-observer variability ${ }^{31}$. Twodimensional speckle-tracking echocardiography has emerged as an alternative technique that analyses motion by tracking natural acoustic reflections and interference patterns within an ultrasonic window. It enables the angle-independent assessment of multidirectional LV strain and differentiates myocardial segments with active contraction from segments which are passively tethered ${ }^{9}$. Myocardial strain derived from 2DSTE has been validated using sono-micrometry ${ }^{32-34}$ and tagged magnetic resonance imaging $^{30,32,35-37}$. Speckle-tracking strain results correlate significantly with tissue Doppler-derived measurements. However, ROC curve analysis has shown that longitudinal and radial strain measured using 2D-STE has a significantly greater area under the curve than TDI strain in differentiating normal and dysfunctional LV segments ${ }^{37}$. 


\section{Clinical implications}

Our study demonstrated that DYS-PAP strongly correlated with postoperative regurgitant volume $(R=0.62, p<0.001)$ and that it was an independent predictor of recurrent $M R$. Indeed, we found that DYS-PAP $\geq 58$ ms predicted recurrence of MR with high sensitivity and specificity. Furthermore, DYS-PAP resulted to be independent of systolic volume, systolic sphericity index and tethering pattern. Indeed, we failed to find significant interactions between DYS-PAP and these parameters.

This may have important clinical implications for the likelihood of postoperative recurrent MR and may help to identify patients who benefit from UMRA.

Compared to data published in the literature in non-ischemic patients ${ }^{9}$, we had lower values of DYS-PAP and this might be attributable to the small number of subjects with QRS >120 ms (19.8\%) which reflects significant mechanical dyssynchrony not associated with electrical dyssynchrony in the majority of patients in our cohort. The small number of patients might also explain the weak correlation between in DYS-PAP and QRS width in our study $(\mathrm{p}=0.10)$. However, our findings are in accordance with recent data showing that the QRS duration does not adequately reflect LV dyssynchrony which may occur only in $30 \%$ of heart failure patients ${ }^{38}$. Published data show that reduction of ischemia by CABG is associated with resynchronization only in a small percentage of patients with a high degree of preoperative dyssynchrony ${ }^{39}$. Accordingly, in our experience, in patients showing recurrent MR with higher preoperative DYSPAP, it did not improve after revascularization whereas it was reduced significantly in patients who did not develop MR postoperatively who had a lower baseline DYS-PAP.

In our study, we found a strong correlation between DYS-PAP and WMSI $(p<0.001)$. Therefore, papillary dyssynchrony by speckle-tracking method resulted to be a volume-independent indicator of LV chamber contractility sensitive to wall motion abnormalities.

It has been demonstrated that the improvement in LV dyssynchrony plays an important role in the development of reverse remodeling after cardiac resynchronization therapy $(C R T)^{40}$ and that, in ischemic MR, local remodeling of the region of the LV supporting the papillary muscles is necessary for the development of functional $\mathrm{MR}^{26}$. Furthermore, strain was reported to correlate with LV Dp/dt and contractile reserve in patients with $\mathrm{MR}^{41,42}$ and speckle-tracking-derived strain was an early marker of LV dysfunction in asymptomatic MR with normal LV ejection fraction ${ }^{43}$.

We might therefore suppose that in patients who did not show recurrent $M R$, preoperative dyssynchrony reflects a reversible state of LV dysfunction and, after surgery, the dyssynchrony of papillary muscles and neighbouring myocardium decreases. The global systolic function and, consequently, closing forces improve and tethering of the mitral apparatus lessens.

In contrast in MR+ patients, impaired contraction of the papillary muscle is not reversed by surgery and the presence of post-repair significant dyssynchrony plays a role 
in the development of recurrent MR. Indeed, uncoordinated regional LV activation leads to a systolic imbalance of the forces acting on papillary muscles, resulting in geometrical changes in the mitral leaflet attachments. In addition, the presence of a significant volume overload due to recurrent MR appears as a factor promoting further LV dilatation and blocking the LV reverse remodeling process.

In other words, it is presumable that re-synchronization in MR-patients successfully interrupts the vicious circle of remodeling and LV dilatation and turns it into the virtuous circle of reverse remodeling and further resynchronization.

Undersized Mitral Ring Annuloplasty has long been considered as an effective approach to relieve CIMR. Nonetheless, although few groups report encouraging results after $U_{M R A^{5}}$, recurrent MR is seen in up to $30 \%$ of patients in other centers ${ }^{44}$. These disappointing results created the need for a better understanding and preoperative assessment of mitral valve configuration and LV geometry and function to improve risk stratification and to allow the identification of patient subgroups that are likely to benefit from this procedure.

The findings of our study advocate routine assessment for LV dyssynchrony, in addition to other echocardiographic parameters, in patients with CIMR undergoing CABG and UMRA. Patients with baseline DYS-PAP $\geq 58 \mathrm{~ms}$ are likely to have MR recurrence and should be considered for concomitant or alternative surgical techniques ${ }^{19,45}$.

\section{Study limitations}

The main limitation of the present study is the lack of information on myocardial viability. This was due to its retrospective nature and to the fact that viability was not routinely documented. The presence of viable myocardium might be responsible for favourable results in the MR-patients. Furthermore, we have no data about graft patency at follow-up and we cannot therefore exclude the possibility that different results might be attributable to abnormalities of perfusion in the MR+ Group. In addition, beyond the lack of information on myocardial viability, there is also no information on the extent of necrotic area. Nonetheless, reappearance of angina, occurrence of acute coronary syndrome and postoperative coronary procedures were not significantly different at follow up between the two groups. This might demonstrate that coronary history during the follow-up did not differ between groups although viability tests and coronarography would have been more informative and accurate regarding the progression of coronary atherosclerosis. All these aspects deserves further evaluation and will be object of an randomized study. Furthermore, the dyssynchrony measurement is based on the time to peak velocity of the papillary muscles on two apical views. This measure is not widely validated and a major concern remains about the reproducibility of this technique. Furthermore this is a retrospective analysis therefore a prospective validation of this method on a prospective dataset in a blinded fashion is necessary. 
Moreover, the PISA method of assessing the severity of ischemic MR is less accurate as the PISA is less likely to be a hemisphere. Adjustments of the aliasing velocity were carried out such that a well-defined hemisphere was obtained. This was done by shifting the baseline toward the direction of the flow, or by lowering the Nyquist limit or both (the latter reduces the wall filter whereas the former does not) ${ }^{15}$.

In addition, as the myocardial movement is basically three-dimensional, thus including rotational movements, two-dimensional speckle tracking may have inherent limitations, especially in patients with dilated LV. However, to our knowledge this is the first report investigating papillary muscle dyssynchrony in CIMR and its impact on predicting recurrent mitral regurgitation after reductive annuloplasty.

\section{Conclusions}

DYS-PAP is strongly correlated with the recurrence of MR after UMRA. A DYS-PAP cutoff value of $\geq 58 \mathrm{~ms}$ resulted to be a useful tool to identify patients in which combined UMRA and CABG are likely to fail. Further larger prospective studies are necessary to confirm our findings. 


\section{References}

1. Bolling SF, Deeb GM, Brunsting LA, Bach DS. Early outcome of mitral valve reconstruction in patients with end-stage cardiomyopathy. The Journal of thoracic and cardiovascular surgery. 1995;109:676-682; discussion 682-683

2. Hung J, Papakostas L, Tahta SA, Hardy BG, Bollen BA, Duran CM, Levine RA. Mechanism of recurrent ischemic mitral regurgitation after annuloplasty: Continued Iv remodeling as a moving target. Circulation. 2004;110:1185-II90

3. Gelsomino S, Lorusso R, De Cicco G, Capecchi I, Rostagno C, Caciolli S, Romagnoli S, Da Broi U, Stefano $P$, Gensini GF. Five-year echocardiographic results of combined undersized mitral ring annuloplasty and coronary artery bypass grafting for chronic ischaemic mitral regurgitation. European heart journal. 2008;29:231-240

4. Dahlberg PS, Orszulak TA, Mullany CJ, Daly RC, Enriquez-Sarano M, Schaff HV. Late outcome of mitral valve surgery for patients with coronary artery disease. The Annals of thoracic surgery. 2003;76:15391487; discussion 1547-1548

5. ten Brinke EA, Klautz RJ, Tulner SA, Verwey HF, Bax JJ, Delgado V, Holman ER, Schalij MJ, van der Wall EE, Braun J, Versteegh MI, Dion RA, Steendijk P. Clinical and functional effects of restrictive mitral annuloplasty at midterm follow-up in heart failure patients. The Annals of thoracic surgery. 2010;90:1913-1920

6. Agricola E, Oppizzi M, Maisano F, De Bonis M, Schinkel AF, Torracca L, Margonato A, Melisurgo G, Alfieri O. Echocardiographic classification of chronic ischemic mitral regurgitation caused by restricted motion according to tethering pattern. European journal of echocardiography: the journal of the Working Group on Echocardiography of the European Society of Cardiology. 2004;5:326-334

7. Dent JM, Spotnitz WD, Nolan SP, Jayaweera AR, Glasheen WP, Kaul S. Mechanism of mitral leaflet excursion. The American journal of physiology. 1995;269:H2100-H2108

8. He S, Fontaine AA, Schwammenthal E, Yoganathan AP, Levine RA. Integrated mechanism for functional mitral regurgitation: Leaflet restriction versus coapting force: In vitro studies. Circulation. 1997;96:1826-1834

9. Tigen K, Karaahmet T, Dundar C, Guler A, Cevik C, Basaran O, Kirma C, Basaran Y. The importance of papillary muscle dyssynchrony in predicting the severity of functional mitral regurgitation in patients with non-ischaemic dilated cardiomyopathy: A two-dimensional speckle-tracking echocardiography study. European journal of echocardiography: the journal of the Working Group on Echocardiography of the European Society of Cardiology. 2010;11:671-676

10. Karvounis HI, Dalamaga EG, Papadopoulos CE, Karamitsos TD, Vassilikos V, Paraskevaidis S, Styliadis IH, Parharidis GE, Louridas GE. Improved papillary muscle function attenuates functional mitral regurgitation in patients with dilated cardiomyopathy after cardiac resynchronization therapy. Journal of the American Society of Echocardiography: official publication of the American Society of Echocardiography. 2006;19:1150-1157

11. Ypenburg C, Lancellotti P, Tops LF, Bleeker GB, Holman ER, Pierard LA, Schalij MJ, Bax JJ. Acute effects of initiation and withdrawal of cardiac resynchronization therapy on papillary muscle dyssynchrony and mitral regurgitation. Journal of the American College of Cardiology. 2007;50:2071-2077

12. Leitman M, Lysyansky P, Sidenko S, Shir V, Peleg E, Binenbaum M, Kaluski E, Krakover R, Vered Z. Twodimensional strain-a novel software for real-time quantitative echocardiographic assessment of myocardial function. Journal of the American Society of Echocardiography: official publication of the American Society of Echocardiography. 2004;17:1021-1029

13. Perk G, Tunick PA, Kronzon I. Non-doppler two-dimensional strain imaging by echocardiography--from technical considerations to clinical applications. Journal of the American Society of Echocardiography: official publication of the American Society of Echocardiography. 2007;20:234-243

14. Carpentier A. Cardiac valve surgery--the "french correction". The Journal of thoracic and cardiovascular surgery. 1983;86:323-337 
15. Zoghbi WA, Enriquez-Sarano M, Foster E, Grayburn PA, Kraft CD, Levine RA, Nihoyannopoulos $P$, Otto CM, Quinones MA, Rakowski H, Stewart WJ, Waggoner A, Weissman NJ. Recommendations for evaluation of the severity of native valvular regurgitation with two-dimensional and doppler echocardiography. Journal of the American Society of Echocardiography: official publication of the American Society of Echocardiography. 2003;16:777-802

16. Schiller NB, Shah PM, Crawford M, DeMaria A, Devereux R, Feigenbaum H, Gutgesell H, Reichek N, Sahn D, Schnittger I, et al. Recommendations for quantitation of the left ventricle by two-dimensional echocardiography. American society of echocardiography committee on standards, subcommittee on quantitation of two-dimensional echocardiograms. Journal of the American Society of Echocardiography: official publication of the American Society of Echocardiography. 1989;2:358-367

17. Kono T, Sabbah HN, Rosman H, Alam M, Jafri S, Goldstein S. Left ventricular shape is the primary determinant of functional mitral regurgitation in heart failure. Journal of the American College of Cardiology. 1992;20:1594-1598

18. Vijayaraghavan G, Boltwood CM, Tei C, Wong M, Shah PM. Simplified echocardiographic measurement of the mitral anulus. American heart journal. 1986;112:985-991

19. Gelsomino S, Lorusso R, Caciolli S, Capecchi I, Rostagno C, Chioccioli M, De Cicco G, Bille G, Stefano P, Gensini GF. Insights on left ventricular and valvular mechanisms of recurrent ischemic mitral regurgitation after restrictive annuloplasty and coronary artery bypass grafting. The Journal of thoracic and cardiovascular surgery. 2008;136:507-518

20. Coats AJ, Shewan LG. Statement on authorship and publishing ethics in the international journal of cardiology. International journal of cardiology. 2011;153:239-240

21. Zhu F, Otsuji Y, Yotsumoto G, Yuasa T, Ueno T, Yu B, Koriyama C, Hamasaki S, Biro S, Kisanuki A, Minagoe S, Levine RA, Sakata R, Tei C. Mechanism of persistent ischemic mitral regurgitation after annuloplasty: Importance of augmented posterior mitral leaflet tethering. Circulation. 2005;112:I3961401

22. Kuwahara E, Otsuji Y, Iguro Y, Ueno T, Zhu F, Mizukami N, Kubota K, Nakashiki K, Yuasa T, Yu B, Uemura T, Takasaki K, Miyata M, Hamasaki S, Kisanuki A, Levine RA, Sakata R, Tei C. Mechanism of recurrent/persistent ischemic/functional mitral regurgitation in the chronic phase after surgical annuloplasty: Importance of augmented posterior leaflet tethering. Circulation. 2006;114:1529-1534

23. Magne J, Pibarot P, Dagenais F, Hachicha Z, Dumesnil JG, Senechal M. Preoperative posterior leaflet angle accurately predicts outcome after restrictive mitral valve annuloplasty for ischemic mitral regurgitation. Circulation. 2007;115:782-791

24. Kanzaki H, Bazaz R, Schwartzman D, Dohi K, Sade LE, Gorcsan J, 3rd. A mechanism for immediate reduction in mitral regurgitation after cardiac resynchronization therapy: Insights from mechanical activation strain mapping. Journal of the American College of Cardiology. 2004;44:1619-1625

25. Appleton CP, Basnight MA, Gonzalez MS. Diastolic mitral regurgitation with atrioventricular conduction abnormalities: Relation of mitral flow velocity to transmitral pressure gradients in conscious dogs. Journal of the American College of Cardiology. 1991;18:843-849

26. Agricola E, Oppizzi M, Galderisi M, Pisani M, Meris A, Pappone C, Margonato A. Role of regional mechanical dyssynchrony as a determinant of functional mitral regurgitation in patients with left ventricular systolic dysfunction. Heart. 2006;92:1390-1395

27. Stoylen A, Heimdal A, Bjornstad K, Wiseth R, Vik-Mo H, Torp H, Angelsen B, Skjaerpe T. Strain rate imaging by ultrasonography in the diagnosis of coronary artery disease. Journal of the American Society of Echocardiography: official publication of the American Society of Echocardiography. 2000;13:10531064

28. Urheim S, Edvardsen T, Torp H, Angelsen B, Smiseth OA. Myocardial strain by doppler echocardiography. Validation of a new method to quantify regional myocardial function. Circulation. 2000;102:1158-1164 
29. Voigt JU, Nixdorff U, Bogdan R, Exner B, Schmiedehausen K, Platsch G, Kuwert T, Daniel WG, Flachskampf FA. Comparison of deformation imaging and velocity imaging for detecting regional inducible ischaemia during dobutamine stress echocardiography. European heart journal. 2004;25:1517-1525

30. Edvardsen T, Skulstad H, Aakhus S, Urheim S, Ihlen H. Regional myocardial systolic function during acute myocardial ischemia assessed by strain doppler echocardiography. Journal of the American College of Cardiology. 2001;37:726-730

31. Castro PL, Greenberg NL, Drinko J, Garcia MJ, Thomas JD. Potential pitfalls of strain rate imaging: Angle dependency. Biomedical sciences instrumentation. 2000;36:197-202

32. Amundsen BH, Helle-Valle $T$, Edvardsen $T$, Torp $H$, Crosby J, Lyseggen E, Stoylen A, Ihlen H, Lima JA, Smiseth OA, Slordahl SA. Noninvasive myocardial strain measurement by speckle tracking echocardiography: Validation against sonomicrometry and tagged magnetic resonance imaging. Journal of the American College of Cardiology. 2006;47:789-793

33. Korinek J, Wang J, Sengupta PP, Miyazaki C, Kjaergaard J, McMahon E, Abraham TP, Belohlavek M. Two-dimensional strain--a doppler-independent ultrasound method for quantitation of regional deformation: Validation in vitro and in vivo. Journal of the American Society of Echocardiography: official publication of the American Society of Echocardiography. 2005;18:1247-1253

34. Toyoda T, Baba H, Akasaka T, Akiyama M, Neishi Y, Tomita J, Sukmawan R, Koyama Y, Watanabe N, Tamano S, Shinomura R, Komuro I, Yoshida K. Assessment of regional myocardial strain by a novel automated tracking system from digital image files. Journal of the American Society of Echocardiography: official publication of the American Society of Echocardiography. 2004;17:1234-1238

35. Notomi Y, Lysyansky P, Setser RM, Shiota T, Popovic ZB, Martin-Miklovic MG, Weaver JA, Oryszak SJ, Greenberg NL, White RD, Thomas JD. Measurement of ventricular torsion by two-dimensional ultrasound speckle tracking imaging. Journal of the American College of Cardiology. 2005;45:2034-2041

36. Roes SD, Mollema SA, Lamb HJ, van der Wall EE, de Roos A, Bax JJ. Validation of echocardiographic twodimensional speckle tracking longitudinal strain imaging for viability assessment in patients with chronic ischemic left ventricular dysfunction and comparison with contrast-enhanced magnetic resonance imaging. The American journal of cardiology. 2009;104:312-317

37. Cho GY, Chan J, Leano R, Strudwick M, Marwick TH. Comparison of two-dimensional speckle and tissue velocity based strain and validation with harmonic phase magnetic resonance imaging. The American journal of cardiology. 2006;97:1661-1666

38. Bleeker GB, Holman ER, Steendijk P, Boersma E, van der Wall EE, Schalij MJ, Bax JJ. Cardiac resynchronization therapy in patients with a narrow qrs complex. Journal of the American College of Cardiology. 2006;48:2243-2250

39. Penicka M, Bartunek J, Lang O, Medilek K, Tousek P, Vanderheyden M, De Bruyne B, Maruskova M, Widimsky $P$. Severe left ventricular dyssynchrony is associated with poor prognosis in patients with moderate systolic heart failure undergoing coronary artery bypass grafting. Journal of the American College of Cardiology. 2007;50:1315-1323

40. Breithardt OA, Sinha AM, Schwammenthal E, Bidaoui N, Markus KU, Franke A, Stellbrink C. Acute effects of cardiac resynchronization therapy on functional mitral regurgitation in advanced systolic heart failure. Journal of the American College of Cardiology. 2003;41:765-770

41. Lee R, Hanekom L, Marwick TH, Leano R, Wahi S. Prediction of subclinical left ventricular dysfunction with strain rate imaging in patients with asymptomatic severe mitral regurgitation. The American journal of cardiology. 2004;94:1333-1337

42. Kim MS, Kim YJ, Kim HK, Han JY, Chun HG, Kim HC, Sohn DW, Oh BH, Park YB. Evaluation of left ventricular short- and long-axis function in severe mitral regurgitation using 2-dimensional strain echocardiography. American heart journal. 2009;157:345-351 
43. Lancellotti P, Cosyns B, Zacharakis D, Attena E, Van Camp G, Gach O, Radermecker M, Pierard LA. Importance of left ventricular longitudinal function and functional reserve in patients with degenerative mitral regurgitation: Assessment by two-dimensional speckle tracking. Journal of the American Society of Echocardiography: official publication of the American Society of Echocardiography. 2008;21:1331-1336

44. Bouma W, van der Horst IC, Wijdh-den Hamer IJ, Erasmus ME, Zijlstra F, Mariani MA, Ebels T. Chronic ischaemic mitral regurgitation. Current treatment results and new mechanism-based surgical approaches. European journal of cardio-thoracic surgery: official journal of the European Association for Cardio-thoracic Surgery. 2010;37:170-185

45. Gelsomino S, Lorusso R, Capecchi I, Rostagno C, Romagnoli S, Bille G, De Cicco G, Tetta C, Stefano P, Gensini GF. Left ventricular reverse remodeling after undersized mitral ring annuloplasty in patients with ischemic regurgitation. The Annals of thoracic surgery. 2008;85:1319-1330 


\section{Chapter 5}

\section{Systolic papillary muscle dyssynchrony}

predicts recurrence of mitral regurgitation in patients with ischemic dilated cardiomyopathy (IDCM) undergoing mitral valve repair

Leen Van Garsse, MD, Sandro Gelsomino, MD, PhD, Orlando Parise, MSc, Fabiana Lucà, MD, EmileCheriex, MD, PhD, Roberto Lorusso, MD, PhD, Enrico Vizzardi, MD, Carmelo Massimiliano Rao, MD, Gian Franco Gensini, MD, Jos Maessen, MD, PhD.

Published in: Echocardiography. 2012 Aug 17. In press. 


\section{Abstract}

Objective: We investigated the impact of papillary muscle dyssynchrony (DYS-PAP) in predicting recurrent mitral regurgitation (MR) in patients with ischemic dilated cardiomyopathy (IDCM) undergoing undersized mitral ring annuloplasty (UMRA).

Methods: One hundred forty-four IDCM patients (left ventricular ejection fraction $<35 \%$ ) in sinus rhythm, undergoing UMRA between January 2001 and December 2010 at three Institutions (University Hospital, Maastricht, the Netherlands; Careggi Hospital, Florence, Italy; Civic Hospital, Brescia, Italy) were recruited. The primary endpoint was the recurrence of MR at the latest echocardiographic control defined as insufficiency $\geq 2+$ in patients with no/trivial MR at discharge.

The assessment of DYS-PAP was performed by applying 2D speckle-tracking imaging.

Results: In patients with MR recurrence DYS-PAP significantly worsened $(84.1 \pm 8.8 \mathrm{~ms}$ vs. $65.4 \pm 8.8 \mathrm{~ms}$ at baseline, $\mathrm{p}<0.001)$ whereas, in patients with no MR recurrence DYS-PAP did not vary ( $22.3 \pm 5.3 \mathrm{~ms}$ vs. $25.9 \pm 7.2 \mathrm{~ms}$ at baseline, $\mathrm{p}=0.8)$. Recurrent MR was positively correlated with DYS-PAP $(p<0.001)$, anterior mitral leaflet tethering angle $\alpha(p<0.001)$ and tethering symmetry index $\alpha / \beta(p<0.001)$. There was no significant correlation between MR recurrence and other echocardiographic parameters. Logistic regression analysis revealed that DYS-PAP (OR: 5.4 [95\% Cl: 3.1-7.7], $\mathrm{p}<0.001$ ), $\alpha$ (OR: 5.0 [95\% Cl: 2.6-6.7], $p<0.001$ ) and $\alpha / \beta$ (OR: 3.9 [95\% Cl: 2.5-5.7], $p<0.001$ ) were predictors of recurrent MR. A DYS-PAP value $\geq 58 \mathrm{~ms}$ predicted recurrence of MR with $100 \%$ sensitivity and $83 \%$ specificity [area under the curve (AUC): 0.92 [95\% Cl: $0.7-1$ ], $\mathrm{p}<0.001]$.

Conclusions: A DYS-PAP cut-off value of $58 \mathrm{~ms}$ is a useful tool to identify patients in which UMRA is likely to fail. Further larger prospective studies are necessary to confirm our findings. 


\section{Introduction}

Functional mitral regurgitation (FMR) is commonly observed in ischemic dilated cardiomyopathy (IDCM) and is associated with a poor prognosis ${ }^{1}$. Undersized mitral ring annuloplasty (UMRA) has been widely used in recent years for the treatment of ischemic FMR. Nonetheless, while some patients do show echocardiographic evidence of valve competence and left ventricular (LV) reverse remodeling ${ }^{2}$, in a large percentage of patients recurrence of MR associated with a persistent or progressive remodeling pattern has clearly been documented over time despite an initially successful surgical repair ${ }^{3}$.

Cardiac dyssynchrony (DYS) is associated with functional deterioration and poor prognosis in advanced systolic heart failure ${ }^{4}$ and is an important determinant of postoperative outcome in patients with severe LV dysfunction ${ }^{5}$. Furthermore, DYS is a strong predictor of LV remodeling after acute myocardial infarction ${ }^{6}$.

Two-dimensional (2D) strain imaging based on novel speckle tracking echocardiography (STE) is a relatively new tool to define regional myocardial strain and to quantify dyssynchrony based on a more robust technique and avoiding angle-dependence ${ }^{7}$.

We testedthehypothesis that systolic papillary muscle dyssynchrony (DYS-PAP) evaluated by $2 \mathrm{D}$ speckle-tracking echocardiography (2D-STE) can predict MR recurrence in patients with IDCM undergoing UMRA.

\section{Materials and methods}

\section{Patients}

Patients with IDCM (LV ejection fraction <35\%) and in sinus rhythm, undergoing UMRA between January 2001 and December 2010 at three Institutions (University Hospital, Maastricht, the Netherlands; Careggi Hospital, Florence, Italy; Civic Hospital, Brescia? Italy) were included in the study. Definitions, inclusion and exclusion criteria were as previously reported ${ }^{8}$. Patients were also excluded if they had residual MR defined as a mitral insufficiency $\geq 2$ at discharge or if echocardiograms were not available/incomplete or images were not appropriate for 2D-STE. One-hundred and forty-four patients met the inclusion criteria and represented the study population, whereas 35 were excluded.

The primary endpoint was the recurrence of MR (insufficiency $\geq 2+$ in patients with no/trivial MR at discharge) at the latest echocardiographic control, performed at a median of 39.3 months (interquartile range 18.9-46.5).

Ethical Committee approval was waived due to the retrospective analysis of the study according to National laws regulating observational retrospective studies (Italian law nr.11960, released on 13/7/2004; Dutch WMO law). However, all patients gave 
their informed consent to access their data for scientific purposes. Preoperative characteristics of the patients are shown in Table 1 . Ninety-eight patients (68.1\%) had $\geq$ moderate recurrent MR at follow-up whereas 46 (31.9\%) did not show recurrent MR at the last control. No difference was detected in preoperative and surgical data between the two groups.

Table 1. Preoperative demographic clinical and surgical data $(n=144)$

\begin{tabular}{|c|c|c|c|c|}
\hline & $\begin{array}{c}\text { All } \\
(n=144)\end{array}$ & $\begin{array}{l}M R- \\
(n=46)\end{array}$ & $\begin{array}{l}M R+ \\
(n=98)\end{array}$ & $\mathrm{p}$ \\
\hline Age, y & $64 \pm 6$ & $67 \pm 8$ & $62 \pm 7$ & 0.07 \\
\hline Gender M/F & $93 / 51(64.6 / 35.4)$ & $32 / 14(69.6 / 30.4)$ & $61 / 37(62.2 / 37.8)$ & 0.4 \\
\hline NYHA class & $3[3-4]$ & $3[3-4]$ & $3[3-4]$ & $>0.9$ \\
\hline Angina & $62(43.0)$ & $21(45.6)$ & $41(41.8)$ & 0.66 \\
\hline Hypertension & 69 (47.9) & $22(47.8)$ & 47 (47.9) & 0.9 \\
\hline Diabetes & 33 (22.9) & 11(23.9) & $22(22.4)$ & 0.83 \\
\hline Smoking & $79(54.8)$ & $22(47.8)$ & $57(58.1)$ & 0.25 \\
\hline Hypercholesterolemia & $86(59.7)$ & $25(54.3)$ & $61(62.2)$ & 0.37 \\
\hline COPD & $15(10.4)$ & $5(10.8)$ & $10(10.2)$ & 0.88 \\
\hline Chronic renal disease & $25(17.3)$ & $8(17.3)$ & $17(17.3)$ & 0.9 \\
\hline Cerebral vascular disease & $13(9.0)$ & $5(10.8)$ & $8(8.1)$ & 0.09 \\
\hline Peripheral vascular disease & $9(6.2)$ & $3(6.5)$ & $6(6.1)$ & 0.9 \\
\hline Familiar history & $81(56.2)$ & $24(52.1)$ & $57(58.1)$ & 0.5 \\
\hline \multicolumn{5}{|l|}{ Myocardial infarction } \\
\hline Inferior/Posterior & $61(42.3)$ & $21(45.6)$ & $40(40.8)$ & 0.6 \\
\hline Anterior/Septal & $15(10.4)$ & $5(10.8)$ & $10(10.2)$ & 0.88 \\
\hline Lateral & $17(11.8)$ & $5(10.8)$ & $12(12.2)$ & 0.83 \\
\hline Combined & $51(35.5)$ & $15(32.6)$ & $36(36.7)$ & 0.63 \\
\hline \multicolumn{5}{|l|}{ Coronary artery disease } \\
\hline Multivessel disease & $65(45.1)$ & $22(47.8)$ & $43(43.8)$ & 0.66 \\
\hline Left Main & $32(22.2)$ & $10(21.7)$ & $22(22.4)$ & $>0.9$ \\
\hline \multicolumn{5}{|l|}{ Surgery } \\
\hline CPB time (min) & 106 [94-123] & 101 [91-117] & 112 [99-125] & 0.06 \\
\hline CCL time (min) & $92[73-114]$ & 89 [70-105] & 97 [81-115] & 0.1 \\
\hline \multicolumn{5}{|l|}{ Mitral Ring } \\
\hline Ring size $(\mathrm{mm})$ & $28[26-30]$ & $28[26-30]$ & $28[26-30]$ & $>0.9$ \\
\hline Carpentier classic & $97(67.4)$ & $30(65.2)$ & $67(68.3)$ & 0.7 \\
\hline Physio ring & $47(32.6)$ & $16(34.7)$ & $31(31.6)$ & 0.7 \\
\hline \multicolumn{5}{|l|}{ CABG } \\
\hline Anastomoses/patient & $2.8 \pm 0.6$ & $2.7 \pm 0.5$ & $2.8 \pm 0.6$ & 0.3 \\
\hline Arterial graft/patient & $1.2 \pm 0.2$ & $1.3 \pm 0.2$ & $1.2 \pm 0.2$ & 0.2 \\
\hline \multicolumn{5}{|l|}{ MR } \\
\hline Grade & $3[3-4]$ & $3[3-4]$ & $3[3-4]$ & $>0.9$ \\
\hline $\mathrm{TA}(\mathrm{cm})$ & $7.4 \pm 0.9$ & $7.4 \pm 0.8$ & $7.5 \pm 0.7$ & 0.4 \\
\hline
\end{tabular}




\begin{tabular}{lcccr}
\hline & $\begin{array}{c}\text { All } \\
(\mathrm{n}=144)\end{array}$ & $\begin{array}{c}\mathrm{MR}- \\
(\mathrm{n}=46)\end{array}$ & $\begin{array}{c}\mathrm{MR}+ \\
(\mathrm{n}=98)\end{array}$ & $\mathrm{p}$ \\
\hline EROA $\left(\mathrm{mm}^{2}\right)$ & $34.4 \pm 8$ & $33.1 \pm 7$ & $36.4 \pm 9$ & 0.1 \\
$\mathrm{RF}(\%)$ & $49.7 \pm 9$ & $46.9 \pm 7$ & $51.5 \pm 9$ & 0.09 \\
$\mathrm{RV}(\mathrm{ml})$ & $55.3 \pm 11$ & $51.7 \pm 10$ & $57.8 \pm 12$ & 0.07 \\
$\mathrm{CH}(\mathrm{cm})$ & $14.1 \pm 3.3$ & $14.3 . \pm 4.0$ & $14.0 \pm 3.1$ & 0.23 \\
$\mathrm{CL}(\mathrm{mm})$ & $3.4 \pm 2.5$ & $3.4 \pm 2.5$ & $3.3 \pm 2.5$ & 0.9 \\
$\mathrm{CD}(\mathrm{mm})$ & $37.3 \pm 6.0$ & $36.6 \pm 5.7$ & $38.0 \pm 6.2$ & 0.5 \\
\hline
\end{tabular}

Normally distributed variables are presented as mean \pm standard deviation; Discrete variables are presented as percentages; Non-normally distributed variables are presented as median [interquartile range].

Abbreviations: $\mathrm{M} / \mathrm{F}=$ Male/Female; NYHA $=$ New York Heart Association; $\mathrm{COPD}=$ Chronic obstructive pulmonary disease $; \mathrm{CPB}=$ Cardiopulmonary bypass; $\mathrm{CCL}=($ Aortic) Cross-clamp; $\mathrm{CABG}=$ Coronary artery bypass grafting; $M R=$ Mitral regurgitation; $T A=$ Tenting area; $E R O A=$ Effective regurgitant orifice area; $R F=$ Regurgitant fraction; $\mathrm{RV}=$ Regurgitant volume; $\mathrm{CH}=$ Coaptation height; $\mathrm{CL}=$ Coaptation length; $\mathrm{CD}=$ Coaptation distance.

\section{Surgery}

Surgery was performed with standard operative techniques, including cardiopulmonary bypass and undersized annuloplasty. Downsizing by two ring sizes was performed in all patients.

All patients underwent associated CABG. For the purposes of this study, complete revascularization was accomplished when at least one graft was placed distally to an approximately $50 \%$ diameter narrowing in each of the three major vascular systems in which arterial narrowing of this severity was noted in a vessel $\geq 1.5 \mathrm{~mm}$ in diameter. Following this definition, $100 \%$ of patients underwent complete revascularization. After cardiopulmonary bypass, a transesophageal echocardiography (TEE) was performed to assess mitral valve repair: leaflet coaptation $\geq 0.5 \mathrm{~cm}, M R \leq 1$ and diastolic $\mathrm{MV}$ area $>2 \mathrm{~cm}^{2}$ was assessed as successful repair.

\section{Standard echocardiographic measurements}

Transthoracic echocardiography (TTE) was performed following a common standard protocol at baseline (within a week before surgery) at discharge, 6 months and at yearly follow-up visits. Two dimensional echoes were carried out using a commercially available ultrasound system IE 33, (Philips Medical System, Amsterdam, The Netherlands). Images were stored in DICOM format and transferred to a workstation for further off-line analysis (Tomtec Imaging system, Unterschleißheim, Germany). Measurements and calculations were made separately by one of the investigators (F.L.). Echocardiographic measurements and calculations were carried out as previously reported $^{8}$. The following quantitative measurements were simultaneously employed to grade the severity of MR: 1) Pulsed Doppler quantitative flow methods; 2) Proximal 
isovelocity surface area (PISA) ${ }^{9}$. Measurements as well as respective thresholds for mild, moderate and severe MR followed the American Society of Echocardiography (ASE) recommendations ${ }^{9}$.

Mitral valve configuration was assessed in mid-systole using the parasternal longaxis and 4-chamber views ${ }^{10}$. The anterior mitral leaflet (AML) tethering angle $\alpha$ (between the annular plane and the basal part of the anterior mitral leaflet), the posterior mitral leaflet (PML) tethering angle $\beta$ (between the angular plane and the basal part of the posterior mitral leaflet) and the bending angle $\gamma$ (between the line, reflecting the bending distance [from the anterior annulus to the bending point created by the tethering of intermediate or strut chordae in the body of the anterior leaflet] and the line from the bending point to the coaptation point) were directly measured with specific software (Philips DICOM Viewer, Philips Medical System, Amsterdam, The Netherlands) ${ }^{10}$. The excursion angles $\alpha_{\mathrm{ex}}$ and $\beta_{\mathrm{ex}}$ were calculated as the difference between AML and PML angles in systole and diastole. The anterior/posterior tethering angle ratio $\alpha / \beta$ was a quantitative measurement of tethering pattern: the more this ratio approached 1 the more symmetric was the tethering ${ }^{8}$.

The tenting area (TA) was measured by the area enclosed between the annular plane and mitral leaflets from the parasternal long-axis view at mid-systole. The coaptation height $(\mathrm{CH})$ was measured as the perpendicular distance between the coaptation point of the mitral leaflets and the line connecting the annular hinge points in the long axis view at end-systole. The coaptation length $(\mathrm{CL})$ was measured as the length of apposition of the anterior and posterior mitral leaflets. The coaptation distance (CD) was measured (along the annular plane) from the anterior leaflet attachment to the point of coaptation.

LV volumes and LV ejection fraction (LVEF) were assessed by the bi-apical Simpson disk method ${ }^{11}$. Sphericity indexes were obtained at end diastole and end systole $\left(\mathrm{SI}_{\mathrm{Dia}}\right.$ and $\mathrm{SI}_{\text {Sys }}$, respectively) as the volume of the LV divided by the volume of a sphere with a diameter equal to the longest axis of the LV measured in the apical view ${ }^{12}$.

The displacement of papillary muscles was quantified as distances from welldefined anatomic landmarks at early and end systole. From the parasternal short-axis view, the geometric chord defined by the intersection of the right and left ventricles ("septal insertions") and the mid-septal perpendicular line were used as references ${ }^{10}$. Lateral and inferior displacements of anterior and posterior papillary muscles were measured as distances from these fixed references. Separation between papillary muscles was directly measured ${ }^{10}$.

The lengths between the anterolateral papillary muscle (ALPM) and posteromedial papillary muscle (PMPM) tips and the contralateral anterior mitral annulus $\left(I_{1}\right.$ and $I_{2}$, respectively) were also measured in mid-systole in the apical 4- and 2-chamber views by using the anterior mitral annulus as a reference point to estimate outward PM displacement $^{13}$. 
The WMSI of the basal and mid-posterior and inferior segments for the PMPM (PMPMWMSI) and basal and mid-lateral and anterior segments for the ALPM (ALPM-WMSI) were also calculated ${ }^{14}$.

\section{Special echocardiographic readings}

Assessment of longitudinal peak systolic strain was performed by applying 2D speckletracking imaging to the apical two- and four-chamber views of the LV. All images were obtained at a frame-rate of 70-90 frames/sec. The LV was divided into six segments in each apical view and the global longitudinal strain (GLS) was obtained in each view ${ }^{14}$. The peak global longitudinal strain $\left(G L S_{\text {peak }}\right)$ was the mean of the peak negative values on the two GLS curves. The assessment of papillary muscle dyssynchrony (DYS-PAP) was performed by applying 2D speckle-tracking imaging to the apical four-chamber view for ALPM and to the apical long-axis view for PMPM ${ }^{15}$.

From an end-systolic single frame, the edge of each papillary muscle was detected on the endocardial cavity and an automated tracking algorithm (free-trace method, Tomtec, Tomtec Imaging system, Unterschleißheim, Germany) followed the papillary muscle from this single frame throughout the cardiac cycle. The value of peak systolic longitudinal strain for each papillary muscle was then determined as anterolateral papillary muscle longitudinal strain (ALPM-LS) and posteromedial papillary muscle longitudinal strain (PMPM-LS). The beginning of the QRS complex was used as the reference point and the time to peak (TTP) systolic longitudinal strain was quantified for each papillary muscle. For the assessment of DYS-PAP, the absolute difference in TTP between anterolateral and posteromedial papillary muscle was calculated (Figure 1).

The reliability of echocardiographic measurements was assessed by calculating intra-observer intervals of agreement of main direct measures used in this study in 20 subjects randomly chosen among the study patients. The Bland-Altman method showed excellent agreement between intra-observer measurements in both low and high values of echocardiographic parameters. (Table 2). 


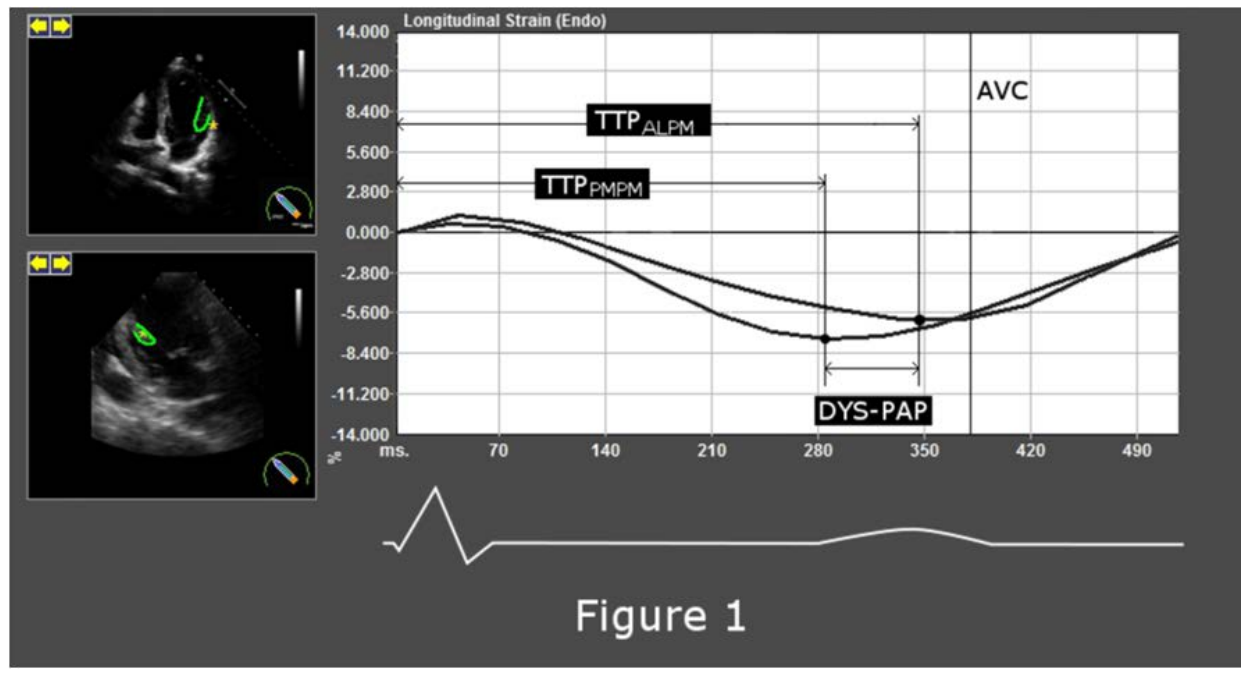

Figure 1. Representative example of measuring papillary muscle dyssynchrony as difference in time to peak (TTP) between anterolateral and posteromedial papillary muscles.

Abbreviations: AVC: Aortic valve closure.

Table 2. Bland -Altman limits of agreement for intra-observer variability.

\begin{tabular}{|c|c|c|c|}
\hline & Bias & Standard Deviation & 95\% Limit of Agreement \\
\hline ALPM-LS & 0.03 & 0.23 & -0.42 to 0.48 \\
\hline PMPM-LS & 0.05 & 0.16 & -0.27 to 0.37 \\
\hline$G_{L S}$ peak & -0.06 & 0.27 & -0.61 to 0.48 \\
\hline DYS-PAP & -0.07 & 0.76 & -1.57 to 1.42 \\
\hline
\end{tabular}

Intra-observer and inter-observer relative differences were $<5 \%$ for all parameters. The Bland-Altman method showed excellent agreement between intra-observer measurements in both low and high values of echocardiographic parameters.

Abbreviations: ALPM-LS = Anterolateral papillary muscle longitudinal strain ; PMPM-LS = Posteromedial papillary muscle longitudinal strain; GLSpeak = Peak Global longitudinal strain; DYS-PAP = Papillary muscle dyssynchrony.

\section{Statistics}

Statistical analysis was performed using a statistical software program (SPSS, version12.0, SPSS Inc. Chicago, IL, USA). Data are presented as mean and standard deviations or as median and interquartile range (IQR) after being controlled for normal distribution by the Kolmogorov-Smirnov test. Data were compared for statistical significance using a t test, Mann-Whitney rank sum test, $\chi 2$ test or Fisher exact test where appropriate. Multiple comparisons were carried out by ANOVA and Kruskal-Wallis tests with Tukey and Dunn post-hoc tests. Pearson's correlation was used to test linear relationships between variables. We assessed, on the entire patient population, univariable regression with postoperative regurgitant volume as a dependent variable and 
other preoperative echocardiographic parameters (end diastolic diameter [EDD], end systolic diameter [ESD], end diastolic volume Index [EDVI], end systolic volume index [ESVI], left ventricular ejection fraction [LVEF], $\mathrm{SI}_{\text {Sys }}, \mathrm{SI}_{\mathrm{Dia}}, \mathrm{TA}, \mathrm{CH}, \mathrm{CL}, \mathrm{CD}, \mathrm{AML}$ tethering angle $\alpha, P M L$ tethering angle $\beta, \alpha / \beta$ ratio, PMPM-WMSI, ALPM-WMSI, DYS-PAP, ALPM-LS, PMPM-LS and $G L S_{\text {peak }}$ ) as independent variables. Variables reaching statistical significance or borderline ( $p \leq 0.1)$ were introduced in multivariable analysis. Multivariate logistic regression with the forward stepwise method was performed to identify preoperative echocardiographic predictors of significant post-repair MR. The goodness of fit of the final logistic regression models was assessed with the HosmerLemeshow (H-M) statistic and predictive accuracy was assessed by the concordance index (c). Optimal cut-off values were determined by the receiver operating characteristic curve (ROC) as the rounding cut-off that gives the maximum sum of sensitivity and specificity. This value should be the shoulder at the top left of the ROC curve. Results were validated using the bootstrap method (1000 iterations).

A $p$ value $<0.05$ was considered statistically significant.

\section{Results}

\section{Leaflet tethering and $L V$ remodeling}

Table 3 shows changes in tethering configuration and LV remodeling before and after surgery.

At preoperative echo patients who developed recurrent MR had lower $\alpha$ excursion angle $(p<0.001)$ and higher $\alpha$ angle $(p=0.003), \alpha / \beta$ ratio $(p<0.001)$, ALPM posterior displacement $(p=0.001)$, ALPM lateral displacement $(p<0.001)$, ALPM apical displacement $(p<0.001)$ and ALPM-WMSI $(p=0.02)$.

At the latest control, in patients with no recurrent $M R, \alpha(p=0.03), \alpha / \beta$ ratio $(p<0.001), \gamma(p=0.01)$ and $\alpha_{\text {ex }}(p=0.03)$ improved whereas $\beta(p=0.04)$ and $\beta_{\text {ex }}(p=0.03)$ significantly worsened. In patients with recurrent $\operatorname{MR} \alpha(p=0.88), \gamma(p=6)$ and $\alpha_{\text {ex }}$ $(p=0.73)$ did not change while $\beta$ and $\beta$ ex (both, $p<0.001$ ) significantly worsened. In these patients the postoperative tethering became more asymmetric $(p<0.001)$. All indices of global LV remodeling significantly improved in MR-patients whereas they worsened in $\mathrm{MR}+$ patients. Ejection fraction increased in MR-patients $(p=0.04)$ while it did not change in $\mathrm{MR}+$ patients $(p=0.85)$.

Regarding local LV remodeling, in patients with no recurrent MR, posterior displacement $(p=0.03)$, lateral displacement $(p=0.002)$ and apical displacement $(p=0.02)$ of ALPM as well as ALPM-WMSI $(p=0.01)$ improved at late follow up, whereas indices of displacement of PMPM significantly worsened (posterior, $p=0.001$; lateral, $p=0.007$; apical, $p=0.04$; PLPM-WMSI, $p=0.007$ ). 
In contrast, in patients with recurrent MR, indices of displacement of PMPM significantly worsened (all, $p<0.001$ ) while ALPM posterior displacement $(p=0.86)$, ALPM lateral displacement $(p=0.80)$, ALPM apical displacement and ALPM-WMSI (both $p>0.9$ ) did not change.

Table 3. Mitral Leaflet Tethering and LV Remodeling.

\begin{tabular}{|c|c|c|c|c|c|c|c|c|c|c|}
\hline & Controls & & $\begin{array}{c}\text { All } \\
(n=144)\end{array}$ & & & $\begin{array}{c}M R- \\
(n=46)\end{array}$ & & & $\begin{array}{c}\mathrm{MR}+ \\
(\mathrm{n}=98)\end{array}$ & \\
\hline & & Baseline & Follow-up & $\mathrm{p}$ & Baseline & Follow-up & $\mathrm{p}$ & Baseline & Follow-up & ( \\
\hline \multicolumn{11}{|l|}{ Mitral tethering } \\
\hline$\alpha\left(\left(^{\circ}\right)\right.$ & $23.2 \pm 3.1$ & $43.1 \pm 6.4^{*}$ & $37.0 \pm 5.5^{*}$ & 0.02 & $37.3 \pm 4.6^{*+}$ & $28.3 \pm 5.5^{* \ddagger}$ & 0.03 & $48.9 \pm 6.8^{*}$ & $47.0 \pm 5.5^{*}$ & 0.88 \\
\hline$\beta\left(\left(^{\circ}\right)\right.$ & $34.1 \pm 5.8$ & $51.3 \pm 5.4^{*}$ & $58.0 \pm 6.6^{*}$ & 0.009 & $49.6 \pm 5.7^{*}$ & $54.6 \pm 7.0^{* \ddagger}$ & 0.04 & $53.1 \pm 5.6^{*}$ & $63.3 \pm 7.9^{*}$ & 0.03 \\
\hline$\alpha / \beta$ & $0.72 \pm 0.2$ & $0.84 \pm 0.1^{*}$ & $0.63 \pm 0.1^{*}$ & $<0.001$ & $0.75 \pm 0.1^{*+}$ & $0.52 \pm 0.1^{* \ddagger}$ & $<0.001$ & $0.92 \pm 0.1^{*}$ & $0.75 \pm 0.1^{*}<$ & $<0.001$ \\
\hline$\gamma\left(\left(^{\circ}\right)\right.$ & $155.1 \pm 8.4$ & $125.4 \pm 5.7^{*}$ & $128 \pm 7.1^{*}$ & 0.61 & $122.1 \pm 3.4^{*}$ & $133 \pm 8.6^{* \ddagger}$ & 0.011 & $127.7 \pm 7.3^{*}$ & $123.6 \pm 5.3^{*}$ & 0.6 \\
\hline$\alpha_{\mathrm{ex}}\left({ }^{\circ}\right)$ & $46.4 \pm 6.7$ & $25.5 \pm 3.0^{*}$ & $31.5 \pm 5.0^{*}$ & 0.03 & $32.1 \pm 4.4^{*+}$ & $40.6 \pm 7.5^{* \ddagger}$ & 0.03 & $20.3 \pm 2.7^{*}$ & $23.9 \pm 4.4^{*}$ & 0.73 \\
\hline$\beta_{\mathrm{ex}}\left(\left(^{\circ}\right)\right.$ & $28.0 \pm 5.6$ & $18.4 \pm 3.5^{*}$ & $10.3 \pm 3.5^{*}$ & 0.001 & $18.2 \pm 3.3^{*}$ & $13.3 \pm 3.1^{* \pm}$ & 0.03 & $19.6 \pm 3.7^{*}$ & $6.3 \pm 3.5^{*}<$ & $<0.001$ \\
\hline \multicolumn{11}{|l|}{ Global LV remodeli } \\
\hline $\mathrm{EDD}(\mathrm{mm})$ & $49.1 \pm 5$ & $69.0 \pm 6^{*}$ & $66.3 \pm 7^{*}$ & 0.74 & $67.3 \pm 6^{*}$ & $52.1 \pm 5^{* \ddagger}$ & 0.004 & $74.0 \pm 8^{*}$ & $80.6 \pm 6^{*}$ & 0.06 \\
\hline $\mathrm{ESD}(\mathrm{mm})$ & $31.7 \pm 4$ & $58.5 \pm 7^{*}$ & $55.7 \pm 6^{*}$ & 0.70 & $58.4 \pm 6^{*}$ & $44.3 \pm 4^{* \ddagger}$ & $<0.001$ & $60.3 \pm 8^{*}$ & $69.1 \pm 0^{*}$ & 0.04 \\
\hline $\operatorname{EDVI}\left(\mathrm{mL} / \mathrm{m}^{2}\right)$ & $55.9 \pm 8$ & $158.3 \pm 24^{*}$ & $155.7 \pm 22^{*}$ & 0.38 & $149.0 \pm 20^{*}$ & $126 \pm 15^{* \ddagger}$ & 0.003 & $166 \pm 29^{*}$ & $180.1 \pm 25^{*}$ & 0.001 \\
\hline $\operatorname{ESVI}\left(\mathrm{mL} / \mathrm{m}^{2}\right)$ & $25.2 \pm 7$ & $113.2 \pm 18^{*}$ & $109.6 \pm 15^{*}$ & 0.55 & $106.2 \pm 14^{*}$ & $78.4 \pm 9^{* \ddagger}$ & $<0.001$ & $119 \pm 23^{*}$ & $138 \pm 20^{*}<$ & $<0.001$ \\
\hline $\mathrm{SI}_{\text {sys }}$ & $0.52 \pm 0.1$ & $0.98 \pm 0.1^{*}$ & $0.92 \pm 0.1^{*}$ & 0.13 & $0.98 \pm 0.1^{*}$ & $0.82 \pm 0.1^{* \ddagger}$ & $<0.001$ & $0.99 \pm 0.2^{*}$ & $1.09 \pm 0.1^{*}$ & 0.04 \\
\hline$S I_{\text {Dia }}$ & $0.64 \pm 0.1$ & $0.84 \pm 0.1^{*}$ & $0.80 \pm 0.1^{*}$ & 0.42 & $0.82 \pm 0.1^{*}$ & $0.70 \pm 0.1^{* \ddagger}$ & $<0.001$ & $0.86 \pm 0.1^{*}$ & $0.91 \pm 0.1^{*}$ & 0.02 \\
\hline LVEF & $0.58 \pm 0.1$ & $0.28 \pm 0.1^{*}$ & $0.29 \pm 0.1^{*}$ & 0.11 & $0.30 \pm 0.1^{*}$ & $0.40 \pm 0.1^{* \ddagger}$ & 0.04 & $0.25 \pm 0.1^{*}$ & $0.24 \pm 0.1^{*}$ & 0.85 \\
\hline \multicolumn{11}{|c|}{ Local LV remodeling } \\
\hline \multicolumn{11}{|c|}{ Posterior Displacement } \\
\hline $\operatorname{ALPM}(\mathrm{cm})$ & $2.1 \pm 0.2$ & $3.1 \pm 0.3^{*}$ & $3.0 \pm 0.3^{*}$ & 0.88 & $2.6 \pm 0.2^{*+}$ & $2.3 \pm 0.2^{* \ddagger}$ & 0.03 & $3.5 \pm 0.4^{*}$ & $3.6 \pm 0.3^{*}$ & 0.86 \\
\hline PMPM $(\mathrm{cm})$ & .2 & $2.7 \pm 0.3^{*}$ & $3.3 \pm 0.5^{*}$ & 0.001 & $2.6 \pm 0.3^{*}$ & $3.3 \pm 0.5^{* \ddagger}$ & 0.001 & $2.8 \pm 0.3^{*}$ & $3.8 \pm 0.5^{*}<$ & $<0.001$ \\
\hline \multicolumn{11}{|l|}{ Lateral Displacemer } \\
\hline $\operatorname{ALPM}(\mathrm{cm})$ & \pm 0.2 & $1.6 \pm 0.4^{*}$ & $1.6 \pm 0.4^{*}$ & $>0.9$ & $1.3 \pm 0.2^{*+}$ & $1.1 \pm 0.4^{* \ddagger}$ & 0.002 & $2.0 \pm 0.5^{*}$ & $2.1 \pm 0.6^{*}$ & 0.8 \\
\hline PMPM (cm) & $0.4 \pm 0.2$ & $1.9 \pm 0.3^{*}$ & $2.5 \pm 0.5^{*}$ & 0.007 & $1.9 \pm 0.4^{*}$ & $2.3 \pm 0.5^{* \ddagger}$ & 0.007 & $1.9 \pm 0.3^{*}$ & $2.6 \pm 0.5^{*}<$ & $<0.001$ \\
\hline \multicolumn{11}{|c|}{ Apical Displacement } \\
\hline $\operatorname{ALPM}\left(I_{1}, \mathrm{~cm}\right)$ & & $4.1 \pm 0.8^{*}$ & $3.9 \pm 0.7^{*}$ & 0.80 & $3.3 \pm 0.7^{*+}$ & $3.0 \pm 05^{* \ddagger}$ & 0.02 & $4.9 \pm 0.9^{*}$ & $4.9 \pm 0.8^{*}$ & $>0.9$ \\
\hline $\operatorname{PMPM}\left(\mathrm{I}_{2}, \mathrm{~cm}\right)$ & $1.9 \pm 0.4$ & $3.7 \pm 0.6^{*}$ & $4.4 \pm 0.6^{*}$ & 0.004 & $3.7 \pm 0.8^{*}$ & $3.9 \pm 0.7^{* \ddagger}$ & 0.04 & $3.9 \pm 0.8^{*}$ & $4.9 \pm 0.8^{*}<$ & $<0.001$ \\
\hline ALPM-WMSI & $1.0 \pm 0$ & $1.6 \pm 0.5^{*}$ & $1.5 \pm 0.5^{*}$ & 0.62 & $1.3 \pm 0.3^{*+}$ & $1.1 \pm 0.3^{* \ddagger}$ & 0.01 & $1.9 \pm 0.7^{*}$ & $1.9 \pm 0.5^{*}$ & $>0.9$ \\
\hline PLPM-WMSI & $1.0 \pm 0$ & $1.4 \pm 0.3^{*}$ & $2.3 \pm 0.4^{*}$ & $<0.001$ & $1.5 \pm 0.3^{*}$ & $2.0 \pm 0.3^{* \ddagger}$ & 0.007 & $1.4 \pm 0.3^{*}$ & $2.6 \pm 0.4^{*}<$ & $<0.001$ \\
\hline
\end{tabular}

Abbreviations: $\alpha=$ Anterior mitral leaflet tethering angle; $\beta=$ posterior mitral leaflet tethering angle; $\gamma=$ bending angle; $\alpha_{\mathrm{ex}}=$ anterior mitral leaflet excursion angle; $\beta_{\mathrm{ex}}=$ posterior mitral leaflet excursion angle; EDD = End Diastolic Diameter; ESD = End Systolic Diameter; EDVI = End Diastolic Volume Index; ESVI = End Systolic Volume Index; $\mathrm{SI}_{\mathrm{Sys}}=$ Systolic Sphericity Index; SI on Fraction; PMPM = Posteromedial papillary Muscle; ALPM = Anterolateral papillary muscle; $\mathrm{WMSI}=\mathrm{Wall}$ Motion Score Index. ${ }^{*}$ Significance vs. controls; ${ }^{\dagger}$ Significance vs. MR+ at baseline; ${ }^{\ddagger}$ Significance vs. MR+ at follow-up. 


\section{Two-dimensional speckle-tracking analysis}

Results of two-dimensional speckle-tracking analysis are shown in Table 4. In patients with MR recurrence PMPM-LS $(p=0.6)$, ALPM-LS $(p=0.78)$ and $G L S_{\text {peak }}(p=0.8)$ had not changed at the last follow up when compared with baseline values. In contrast, DYSPAP significantly worsened ( $<<0.001$, [Figure 2 A] ) at the last echocardiographic control compared to baseline values. In contrast, in patients without MR recurrence, PMPM-LS $(p<0.001)$, ALPM-LS $(p<0.001)$ and $G^{2} S_{\text {peak }}(p=0.001)$ improved whereas DYSPAP did not vary ( $p=0.8$, [Figure $2 \mathrm{~B}$ ]) at the last postoperative echocardiographic controls. All these indices were significantly higher (all, $p<0.001$ ) in patients with recurrent MR compared to the patients without MR.

Table 4. Mitral Leaflet Tethering and LV Remodeling.

\begin{tabular}{|c|c|c|c|c|c|c|c|c|c|c|}
\hline & \multirow[t]{2}{*}{ Controls } & \multicolumn{2}{|c|}{$\begin{array}{c}\text { All } \\
(n=144)\end{array}$} & \multicolumn{4}{|c|}{$\begin{array}{l}\text { MR - } \\
(n=46)\end{array}$} & \multicolumn{3}{|c|}{$\begin{array}{c}\mathrm{MR}+ \\
(\mathrm{n}=98)\end{array}$} \\
\hline & & Baseline & Follow-up & $\mathrm{p}$ & Baseline & Follow-up & $p$ & Baseline & Follow-up & $\mathrm{p}$ \\
\hline PMPM-LS (\%) & $-19.4 \pm 5.9$ & $-7.4 \pm 2.9^{*}$ & $9.1 \pm 3.9^{*}$ & 0.3 & $-9.7 \pm 3.5^{* \dagger}$ & $-15.0 \pm 5.6^{* \ddagger}$ & $<0.001$ & $-5.2 \pm 2.5^{*}$ & $-3.2 \pm 2.2^{*}$ & 0.06 \\
\hline ALPM-LS (\%) & $-22.3 \pm 7.1$ & $8.2 \pm 3.2^{*}$ & $11.1 \pm 4.4^{*}$ & 0.1 & $-12.4 \pm 3.2^{* \dagger}$ & $-18.9 \pm 6.7^{* \ddagger}$ & $<0.001$ & $-4.6 \pm 3.3^{*}$ & $-3.4 \pm 2.2^{*}$ & 0.09 \\
\hline $\mathrm{GLS}_{\text {peak }}(\%)$ & $-20.7 \pm 7.0$ & $8.3 \pm 3.3^{*}$ & $11.2 \pm 4.0^{*}$ & 0.1 & $-10.1 \pm 4.3^{*+}$ & $-16.6 \pm 5.5^{* \ddagger}$ & 0.001 & $-6.5 \pm 2.3^{*}$ & $-5.8 \pm 2.4^{*}$ & 0.3 \\
\hline DYS-PAP (ms) & $4.8 \pm 2.1$ & $45.8 \pm 7.6^{*}$ & $53.2 \pm 8.1^{*}$ & 0.05 & $25.9 \pm 7.2^{* \dagger}$ & $22.3 \pm 7.3^{* \ddagger}$ & 0.8 & $65.4 \pm 8.2^{*}$ & $84.1 \pm 8.8^{*}$ & $<0.001$ \\
\hline
\end{tabular}

Abbreviations: ALPM-LS = Anterolateral papillary muscle longitudinal strain; PMPM-LS = Posteromedial papillary muscle longitudinal strain; $G L S_{\text {peak }}$ = Peak Global longitudinal strain; DYS-PAP = Papillary muscle . Significance vs. controls; ${ }^{\dagger}$ Significance vs. MR+ at baseline; ${ }^{\ddagger}$ Significance vs. MR+ at follow-up. 


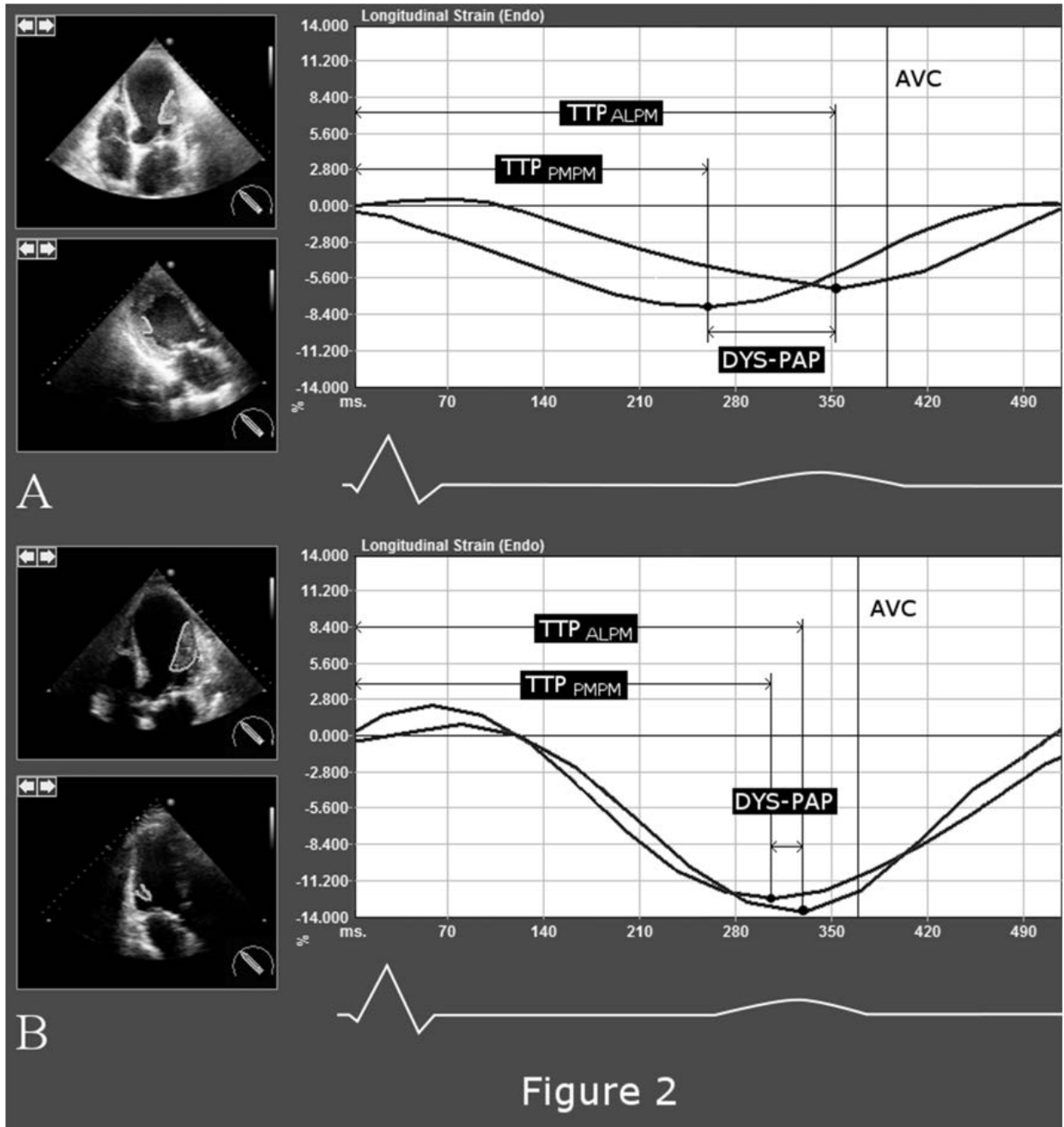

Figure 2. A) Papillary muscle dyssynchrony (DYS-PAP) at follow-up in a patient showing recurrent MR. B) Postoperative DYS-PAP in a patient without MR.

\section{Echocardiographic predictors of recurrent mitral regurgitation}

Recurrent MR was positively correlated with DYS-PAP $(p<0.001), \alpha$ angle $(p<0.001)$ and $\alpha / \beta$ ( $p<0.001[$ Table 5]). There was no significant correlation between MR recurrence and other echocardiographic parameters.

Logistic regression analysis revealed that DYS-PAP (OR: $5.4,95 \% \mathrm{Cl}: 3.1-7.7$, $\mathrm{p}<0.001$ ), AML tethering angle $\alpha$ (OR: 5.0, 95\% Cl: 2.6-6.7, $\mathrm{p}<0.001)$ and tethering symmetry (OR: $3.9,95 \% \mathrm{Cl}: 2.5-5.7, \mathrm{p}<0.001)$ were predictors of recurrent MR (Table 5). The model proved to be reliable $(H L, p=0.8)$ and accurate $(c=0.7)$. 
ROC analysis was performed to assess the utility of DYS-PAP to predict moderate or moderate-to-severe recurrent MR (Figure 3). A DYS-PAP value $\geq 58 \mathrm{~ms}$ predicted recurrence of MR with $100 \%$ sensitivity and $83 \%$ specificity (AUC $0.92,95 \% \mathrm{Cl}: 0.7-1$, $p<0.001$ ). Anterior tethering angle $\alpha$ had $95 \%$ sensitivity and $80 \%$ specificity with an optimal cut-off of $\geq 39.5^{\circ}$ (AUC $0.86,95 \% \mathrm{Cl} 0.72-0.95, p<0.001$ ); $\alpha / \beta$ with a cut-off of $\geq$ 0.75 had $88 \%$ sensitivity and $79 \%$ specificity (AUC $0.82,95 \% \mathrm{Cl} 0.70-0.93, \mathrm{p}<0.001$ ).

Table 5. Predictors of recurrent MR

\begin{tabular}{lccccc}
\hline & \multicolumn{2}{c}{ Univariable } & \multicolumn{3}{c}{ Multivariable } \\
& $\mathrm{R}$ & $\mathrm{p}$ & $\mathrm{OR}$ & $95 \% \mathrm{Cl}$ & $\mathrm{P}$ \\
\hline$\alpha($ o) & 0.78 & $<0.001$ & 5.0 & $2.6-6.7$ & $<0.001$ \\
$\alpha / \beta$ & 0.72 & $<0.001$ & 3.9 & $2.5-5.7$ & $<0.001$ \\
DYS-PAP (ms) & 0.84 & $<0.001$ & 5.4 & $3.1-7.7$ & $<0.001$ \\
\hline
\end{tabular}

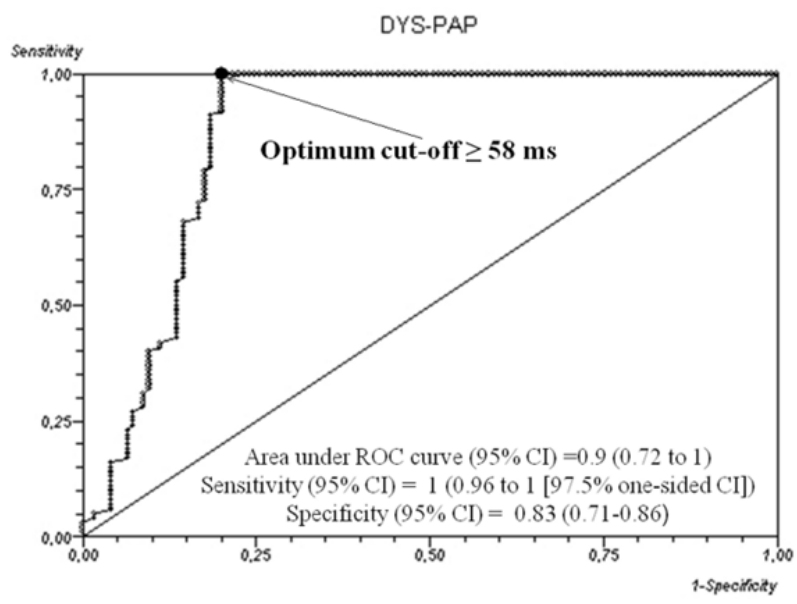

Figure 3. Receiver operating characteristic (ROC) curve demonstrating the predictive value of papillary muscle dyssynchrony for recurrence of mitral regurgitation after undersized mitral ring annuloplasty in patients with ischemic dilated cardiomyopathy. The cut-off value is marked in the plot by a dot.

\section{Discussion}

\section{General considerations}

Mitral valve repair has been preferred to replacement in patients with CIMR and LV dysfunction because of its positive effect with respect to preservation of ventricular function and improved survival ${ }^{16,17}$. Nonetheless, concern has been raised about the 
high incidence of recurrent MR following restrictive annuloplasty in CIMR patients ${ }^{18}$ which has been reported to range between $33 \%$ and $70 \%$ at 5 years ${ }^{3,19}$. Although predictors of reappearance or deterioration of MR have been related to preoperative mitral valve anatomical features, or to LV geometry and dimensions ${ }^{20,21}$, it seems unquestionable that, also in the presence of intraoperative successful repair, recurrence of MR still represents a disappointing and not unusual complication in CIMR patients submitted to mitral valve annuloplasty ${ }^{3,8,19}$. For this reason, much of current research in this area has been focused on identifying predictive factors of recurrent MR in order to select patients who can really benefit from annuloplasty. Functional MR and intraventricular dyssynchrony are common findings in patients with heart failure and they are associated with a poor prognosis ${ }^{4,5}$. The dyssynchronic contractions of LV segments are the leading causes of functional MR in these patients and are independently related to severity of functional $\mathrm{MR}^{22}$. In our study we investigated the impact of papillary muscle dyssynchrony (DYS-PAP) on the recurrence of MR in patients with ischemic dilated cardiomyopathy (IDCM) undergoing UMRA by 2-D speckle-tracking echocardiography (2D-STE) which allows differentiation of myocardial segments with active contractions from segments which are passively tethered ${ }^{7}$.

\section{Key findings}

Our study demonstrated that papillary muscle dyssynchrony was the strongest independent predictor of recurrent MR. Indeed, we found that DYS-PAP $\geq 58$ ms predicted recurrence of MR with $100 \%$ sensitivity and $83 \%$ specificity. Anterior tethering angle $\alpha$ had $95 \%$ sensitivity and $80 \%$ specificity with an optimal cut-off of $\geq 39.5^{\circ}$ and tethering symmetry with a cut-off of $\geq 0.75$ had $88 \%$ sensitivity and $79 \%$ specificity.

Penicka et al $^{5}$ have recently demonstrated that high-degree LV dyssynchrony hinders LV pump function recovery in patients with ischemic heart failure undergoing myocardial revascularization.

Myocardial ischemia is one of the major causes of LV dyssynchrony ${ }^{23}$ and one may speculate that correction of ischemia by CABG surgery should resynchronize LV contractions.

In the present study, patients with severe preoperative dyssynchrony did not show a reduction in DYS-PAP by CABG surgery, thus confirming that extensive LV dyssynchrony cannot be reversed by revascularization and that persistent high-degree dyssynchrony is associated with MR recurrence. Furthermore, in patients with significant $M R$ at follow-up, DYS-PAP significantly worsened. In these patients volume overload resulting from MR leads to further ventricular dilatation, LV dysfunction and DYS-PAP which subsequently leads to further regurgitation. The persistent mechanical dyssynchrony between LV segments supporting the papillary muscles also produces uncoordinated regional LV mechanical activation in these segments and decreases LV contraction efficiency and closing forces, thereby impairing mitral valve tenting and resulting 
in further geometric changes in mitral leaflet attachments and a worsening of mitral leaflet tethering.

We might postulate that DYS-PAP perpetuates the vicious circle whereby ventricular dilatation, LV dysfunction and changes in LV geometry promote MR and in turn MR promotes dilatation, dysfunction and geometrical remodeling. In contrast, in patients with low baseline DYS-PAP, the preserved synchronic contractions of papillary muscles might turn the vicious cycle into the virtuous cycle of normalization of LV function and reverse remodeling. Thus, preoperative DYS-PAP might represent a landmark of advanced myocardial damage in IDCM and a sign of irreversible progression of the disease. Studies on this topic are ongoing.

\section{Clinical implications}

The findings of our study advocate routine assessment for LV dyssynchrony, in addition to other echocardiographic parameters, in patients with IDCM undergoing CABG and UMRA. In these patients the presence of severe baseline DYS-PAP $\geq 58 \mathrm{~ms}$ was associated with high MR recurrence. Therefore, noninvasive testing to assess papillary dyssynchrony should be performed before surgery to guide patient selection. Patients with severe dyssynchrony might be considered for concomitant or alternative surgical techniques $^{24,25}$.

\section{Study limitations}

Our study findings should be viewed in light of some inherent limitations. First, viability testing was not performed in these patients. Therefore, the absence of viable myocardium might be responsible for irreversible dyssynchrony in patients showing unfavorable results. This issue deserves further investigation. Second, postoperative evaluation of the coronary status was not assessed. It would have been helpful to differentiate between surgical failure (valve repair and CABG) and the progress of the coronary disease. Third, the PISA method of assessing the severity of ischemic MR is less accurate as the PISA is less likely to be a hemisphere. Adjustments of the aliasing velocity were carried out such that a well-defined hemisphere was obtained. This was done by shifting the baseline toward the direction of the flow, or by lowering the Nyquist limit or both (the latter reduces the wall filter whereas the former does not) ${ }^{9}$. Furthermore, as the PISA method overestimates MR in patients with eccentric jets, we therefore also employed Pulsed Doppler quantitative flow methods and, in case of contradictory results, PISA was chosen in the presence of central jet or calcific mitral valve/mitral annulus, whereas pulsed Doppler quantitative flow methods were preferred when the jet was eccentric or multiple. 
Finally, as the myocardial movement is basically three-dimensional, thus including rotational movements, two-dimensional speckle tracking may have inherent limitations, especially in patients with dilated LV.

\section{Conclusions}

DYS-PAP is strongly correlated with the recurrence of MR. A DYS-PAP cut-off value of $58 \mathrm{~ms}$ is a useful tool to identify patients in whom UMRA is likely to fail. Further larger prospective studies are necessary to confirm our findings. 


\section{References}

1. Di Mauro M, Di Giammarco G, Vitolla G, Contini M, laco AL, Bivona A, Weltert L, Calafiore AM. Impact of no-to-moderate mitral regurgitation on late results after isolated coronary artery bypass grafting in patients with ischemic cardiomyopathy. The Annals of thoracic surgery. 2006;81:2128-2134

2. Bax JJ, Braun J, Somer ST, Klautz R, Holman ER, Versteegh MI, Boersma E, Schalij MJ, van der Wall EE, Dion RA. Restrictive annuloplasty and coronary revascularization in ischemic mitral regurgitation results in reverse left ventricular remodeling. Circulation. 2004;110:II103-II108

3. Hung J, Papakostas L, Tahta SA, Hardy BG, Bollen BA, Duran CM, Levine RA. Mechanism of recurrent ischemic mitral regurgitation after annuloplasty: Continued IV remodeling as a moving target. Circulation. 2004;110:1185-II90

4. Cho GY, Song JK, Park WJ, Han SW, Choi SH, Doo YC, Oh DJ, Lee Y. Mechanical dyssynchrony assessed by tissue doppler imaging is a powerful predictor of mortality in congestive heart failure with normal qrs duration. Journal of the American College of Cardiology. 2005;46:2237-2243

5. Penicka M, Bartunek J, Lang O, Medilek K, Tousek P, Vanderheyden M, De Bruyne B, Maruskova M, Widimsky $P$. Severe left ventricular dyssynchrony is associated with poor prognosis in patients with moderate systolic heart failure undergoing coronary artery bypass grafting. Journal of the American College of Cardiology. 2007;50:1315-1323

6. Turan B, Yilmaz F, Karaahmet T, Tigen K, Mutlu B, Basaran Y. Role of left ventricular dyssynchrony in predicting remodeling after st elevation myocardial infarction. Echocardiography. 2012;29:165-172

7. Nesser HJ, Winter S. Speckle tracking in the evaluation of left ventricular dyssynchrony. Echocardiography. 2009;26:324-336

8. Gelsomino S, Lorusso R, Caciolli S, Capecchi I, Rostagno C, Chioccioli M, De Cicco G, Bille G, Stefano P, Gensini GF. Insights on left ventricular and valvular mechanisms of recurrent ischemic mitral regurgitation after restrictive annuloplasty and coronary artery bypass grafting. The Journal of thoracic and cardiovascular surgery. 2008;136:507-518

9. Zoghbi WA, Enriquez-Sarano M, Foster E, Grayburn PA, Kraft CD, Levine RA, Nihoyannopoulos P, Otto CM, Quinones MA, Rakowski H, Stewart WJ, Waggoner A, Weissman NJ. Recommendations for evaluation of the severity of native valvular regurgitation with two-dimensional and doppler echocardiography. Journal of the American Society of Echocardiography: official publication of the American Society of Echocardiography. 2003;16:777-802

10. Gelsomino S, Van Garsse L, Luca F, Lorusso R, Cheriex E, Rao CM, Caciolli S, Vizzardi E, Crudeli E, Stefano $P$, Gensini GF, Maessen J. Impact of preoperative anterior leaflet tethering on the recurrence of ischemic mitral regurgitation and the lack of left ventricular reverse remodeling after restrictive annuloplasty. Journal of the American Society of Echocardiography: official publication of the American Society of Echocardiography. 2011;24:1365-1375

11. Schiller NB, Shah PM, Crawford M, DeMaria A, Devereux R, Feigenbaum H, Gutgesell H, Reichek N, Sahn D, Schnittger I, et al. Recommendations for quantitation of the left ventricle by two-dimensional echocardiography. American society of echocardiography committee on standards, subcommittee on quantitation of two-dimensional echocardiograms. Journal of the American Society of Echocardiography: official publication of the American Society of Echocardiography. 1989;2:358-367

12. Kono T, Sabbah HN, Rosman H, Alam M, Jafri S, Goldstein S. Left ventricular shape is the primary determinant of functional mitral regurgitation in heart failure. Journal of the American College of Cardiology. 1992;20:1594-1598

13. Zhang H, Otsuji Y, Uemura T, Yu B, Takeuchi M, Hamasaki S, Miyata M, Kisanuki A, Minagoe S, Levine RA, Tei C. Different mechanisms of ischemic mitral regurgitation in patients with inferior and anterior myocardial infarction. J Echocardiogr. 2008;8:74-83 
14. Cerqueira MD, Weissman NJ, Dilsizian V, Jacobs AK, Kaul S, Laskey WK, Pennell DJ, Rumberger JA, Ryan T, Verani MS. Standardized myocardial segmentation and nomenclature for tomographic imaging of the heart: A statement for healthcare professionals from the cardiac imaging committee of the council on clinical cardiology of the american heart association. Circulation. 2002;105:539-542

15. Tigen K, Karaahmet T, Dundar C, Guler A, Cevik C, Basaran O, Kirma C, Basaran Y. The importance of papillary muscle dyssynchrony in predicting the severity of functional mitral regurgitation in patients with non-ischaemic dilated cardiomyopathy: A two-dimensional speckle-tracking echocardiography study. European journal of echocardiography: the journal of the Working Group on Echocardiography of the European Society of Cardiology. 2010;11:671-676

16. LaPar DJ, Kron IL. Should all ischemic mitral regurgitation be repaired? When should we replace? Current opinion in cardiology. 2011;26:113-117

17. Bouma W, van der Horst IC, Wijdh-den Hamer IJ, Erasmus ME, Zijlstra F, Mariani MA, Ebels T. Chronic ischaemic mitral regurgitation. Current treatment results and new mechanism-based surgical approaches. European journal of cardio-thoracic surgery: official journal of the European Association for Cardio-thoracic Surgery. 2010;37:170-185

18. Shiota M, Gillinov AM, Takasaki K, Fukuda S, Shiota T. Recurrent mitral regurgitation late after annuloplasty for ischemic mitral regurgitation. Echocardiography. 2011;28:161-166

19. ten Brinke EA, Klautz RJ, Tulner SA, Verwey HF, Bax JJ, Delgado V, Holman ER, Schalij MJ, van der Wall $\mathrm{EE}$, Braun J, Versteegh MI, Dion RA, Steendijk P. Clinical and functional effects of restrictive mitral annuloplasty at midterm follow-up in heart failure patients. The Annals of thoracic surgery. 2010;90:1913-1920

20. Calafiore AM, Di Mauro M, Gallina S, Di Giammarco G, laco AL, Teodori G, Tavarozzi I. Mitral valve surgery for chronic ischemic mitral regurgitation. The Annals of thoracic surgery. 2004;77:1989-1997

21. De Bonis M, Lapenna E, Verzini A, La Canna G, Grimaldi A, Torracca L, Maisano F, Alfieri O. Recurrence of mitral regurgitation parallels the absence of left ventricular reverse remodeling after mitral repair in advanced dilated cardiomyopathy. The Annals of thoracic surgery. 2008;85:932-939

22. Hung CL, Tien SL, Lo Cl, Hung TC, Yeh HI, Wang YS. The incremental value of regional dyssynchrony in determining functional mitral regurgitation beyond left ventricular geometry after narrow qrs anterior myocardial infarction: A real time three-dimensional echocardiography study. Echocardiography. 2011;28:665-675

23. Forrester JS, Wyatt HL, Da Luz PL, Tyberg JV, Diamond GA, Swan HJ. Functional significance of regional ischemic contraction abnormalities. Circulation. 1976;54:64-70

24. Borger MA, Alam A, Murphy PM, Doenst T, David TE. Chronic ischemic mitral regurgitation: Repair, replace or rethink? The Annals of thoracic surgery. 2006;81:1153-1161

25. Gelsomino S, Lorusso R, Capecchi I, Rostagno C, Romagnoli S, Bille G, De Cicco G, Tetta C, Stefano P, Gensini GF. Left ventricular reverse remodeling after undersized mitral ring annuloplasty in patients with ischemic regurgitation. The Annals of thoracic surgery. 2008;85:1319-1330 


\section{Chapter 6}

Tethering symmetry reflects advanced left ventricular mechanical dyssynchrony in patients with ischemic mitral regurgitation undergoing restrictive mitral valve repair

Leen Van Garsse, MD, Sandro Gelsomino, MD, PhD, EmileCheriex, MD, PhD, Fabiana Lucà, MD, Carmelo Massimiliano Rao, MD, Orlando Parise, MS, Gian Franco Gensini, MD, Jos Maessen, MD, PhD.

Published in: Ann Thorac Surg. 2012 Nov;94(5):1418-28. 


\section{Abstract}

Background: We evaluated the papillary muscle systolic dyssynchrony (DYS-PAP) using two-dimensional speckle tracking echocardiography (2D-STE) in patients with chronic ischemic mitral regurgitation (CIMR) showing different preoperative leaflet pattern and investigated the impact of baseline tethering pattern in the prediction of significant post-repair desynchronized papillary muscle contraction.

Methods: We recruited 152 CIMR consecutive patients $(64.4 \%$ male, mean age $65.9 \pm$ 7.1 yrs.) who survived coronary artery bypass grafting (CABG) and undersized mitral ring annuloplasty (UMRA), performed between 2001 and 2010. The assessment of DYS-PAP was performed preoperatively and at follow up (median 41.5 months [IQR 2361]) by 2D-STE in the apical four-chamber view for anterolateral papillary muscle (ALPM) and apical long-axis view for posteromedial papillary muscle (PMPM). Based on the cut-off value (anterior/posterior tethering angle ratio $\alpha / \beta \geq 0.76$ ) patients were classified in two Groups: symmetrical (Group $1, n=73$, mean $\alpha / \beta=0.81 \pm 0.6$ ) and asymmetrical preoperative tethering pattern (Group 2, $n=79$, mean $\alpha / \beta=0.66 \pm 0.4$ ).

Results: Recurrent MR occurred in $67.1 \%(n=49)$ in Group 1 vs. 3.8\% $(n=3)$ in Group2 $(p<0.001)$.

Comparing both Groups at baseline, patients in Group 1 had higher DYS-PAP (57.7 \pm 5.3 vs. $29.8 \pm 2.4 \mathrm{~ms}$ in Group 2, $\mathrm{p}<0.001$ ) which significantly worsened at follow up (78.1 $\pm 8.8 \mathrm{~ms}, \mathrm{p}<0.001$ vs. baseline) whereas in Group 2 it improved ( $26.6 \pm 6.0 \mathrm{~ms}$, $\mathrm{p}<0.001$ vs. baseline). Tethering symmetry significantly correlated with DYS-PAP $(r=0.90, p<0.001)$ and it was a strong multivariable predictor of significant postoperative DYS-PAP (OR 4.2 [95\%Cl: 3.4-5.2], p<0.001).

Conclusions: Tethering symmetry is an easy and immediate tool to identify CIMR patients with advanced DYS-PAP who are unlikely to benefit from mitral repair with UMRA. 


\section{Introduction}

Chronic Ischemic Mitral Regurgitation (CIMR) remains one of the most complex and unresolved aspects of ischemic heart disease. Because of the unsatisfactory results of current strategies ${ }^{1,2}$ CIMR is becoming the focus of an increasing amount of cardiovascular research mainly focused on better understanding pathophysiological mechanisms underlying CIMR and their impact on postoperative results ${ }^{3}$. In particular, more attention has been drawn to the preoperative tethering pattern to predict MR recurrence after undersizing mitral ring annuloplasty (UMRA) and to establish whether a specific preoperative leaflet configuration is related to unfavourable outcomes ${ }^{4-6}$.

Recent evidence suggests that dyssynchronous papillary muscle activation may be a contributing factor in functional mitral regurgitation ${ }^{7}$ and it has been reported to be useful in detecting subclinical deterioration in LV function ${ }^{8}$. Nonetheless, no information exists about papillary muscle systolic dyssynchrony (DYS-PAP) in CIMR and little is known about the relationship between DYS-PAP and tethering pattern in CIMR patients and whether the persistence of dyssynchronous papillary muscle activation might be related to a specific leaflet configuration.

In this study we evaluated the DYS-PAP with two-dimensional speckle tracking echocardiography (2D-STE) in CIMR patients and we investigated the impact of baseline tethering pattern on the prediction of significant post-repair desynchronized papillary muscle contraction.

\section{Material and methods}

\section{Subjects}

The Ethics Committee approved the study and waived the need for patient consent according to the national law regulating observational retrospective studies (Dutch WMO law). However, all patients gave their informed consent to access their data for scientific purposes.

We retrospectively evaluated patients with CIMR referred to our Institution (University Hospital of Maastricht, Maastricht, The Netherlands) by five satellite hospitals (Heerlen, Roermond, Sittard, Venlo and Weert, The Nederlands) between 2001 and 2010.

Inclusion Criteria were ${ }^{6,9}:$ (1) mild-to-severe MR with prior myocardial infarction $>16$ days; (2) 75\% or greater stenosis of at least one coronary vessel; (3) a corresponding regional wall motion abnormality; (4) restricted-motion type IIIb leaflet dysfunction with or without annular dilatation.

Exclusion criteria were: (1) mitral valve replacement; (2) death; (3) persistent/residual $M R$ ( $M R \geq$ moderate at discharge); (4) degenerative or other non- 
ischemic etiology; (5) acute ischemic mitral regurgitation; (6) additional mitral valve repair procedures; (7) other valvular or congenital heart diseases; (8) previous cardiac surgery/percutaneous transluminal coronary angioplasty; (9) atrial fibrillation and (10) incomplete/unavailable echoes for strain analysis. The final study population consisted of 152 CIMR patients (Figure 1).

Recurrence of MR was defined as insufficiency $\geq$ moderate (in patients with no/trivial MR at discharge) at the last echocardiographic control performed at a median of 41.5 months [interquartile range (IQR) 23-61].

\section{Surgery}

Patients with moderate or severe CIMR (effective regurgitant orifice area [EROA]> $20 \mathrm{~mm}^{2}$ and regurgitant volume [RV] $>30 \mathrm{~mL}$ ) were scheduled for operation. When MR was moderate, surgery was indicated (1) in the presence of a dilated left ventricle (end-diastolic volume $>110 \mathrm{~mL} / \mathrm{m} 2)$ or low LV ejection fraction $(<0.35)$, as in the case of dilated cardiomyopathy ${ }^{10}$.

All patients underwent complete revascularization. Mitral annuloplasty was performed with standard operative techniques, including cardiopulmonary bypass and undersized annuloplasty rigid ring placement (Carpentier-Edwards Classic; Edwards LifeSciences, Irvine, CA). The ring size was determined by standard measurements of the inter-trigonal distance and anterior leaflet height. Downsizing by 2 ring sizes was performed in all patients. A successful repair was assessed as leaflet coaptation of 0.8 $\mathrm{cm}$ or more, MR of 1 or less and a systolic MV area exceeding $2 \mathrm{~cm}^{2}$ at intraoperative transesophageal echocardiography.

\section{Echocardiography}

Transthoracic echocardiography (TTE) was performed following a common standard protocol at baseline (within a week before surgery) at discharge, 6 months and at yearly follow-up visits. Exams were carried out using a commercially available ultrasound system IE 33, (Philips Medical System, Amsterdam, The Netherlands). Images were stored in DICOM format and transferred to a workstation for further offline analysis (Tomtec Imaging system, Unterschleißheim, Germany). Measurements and calculations were made separately by one of the investigators (F.L.). 


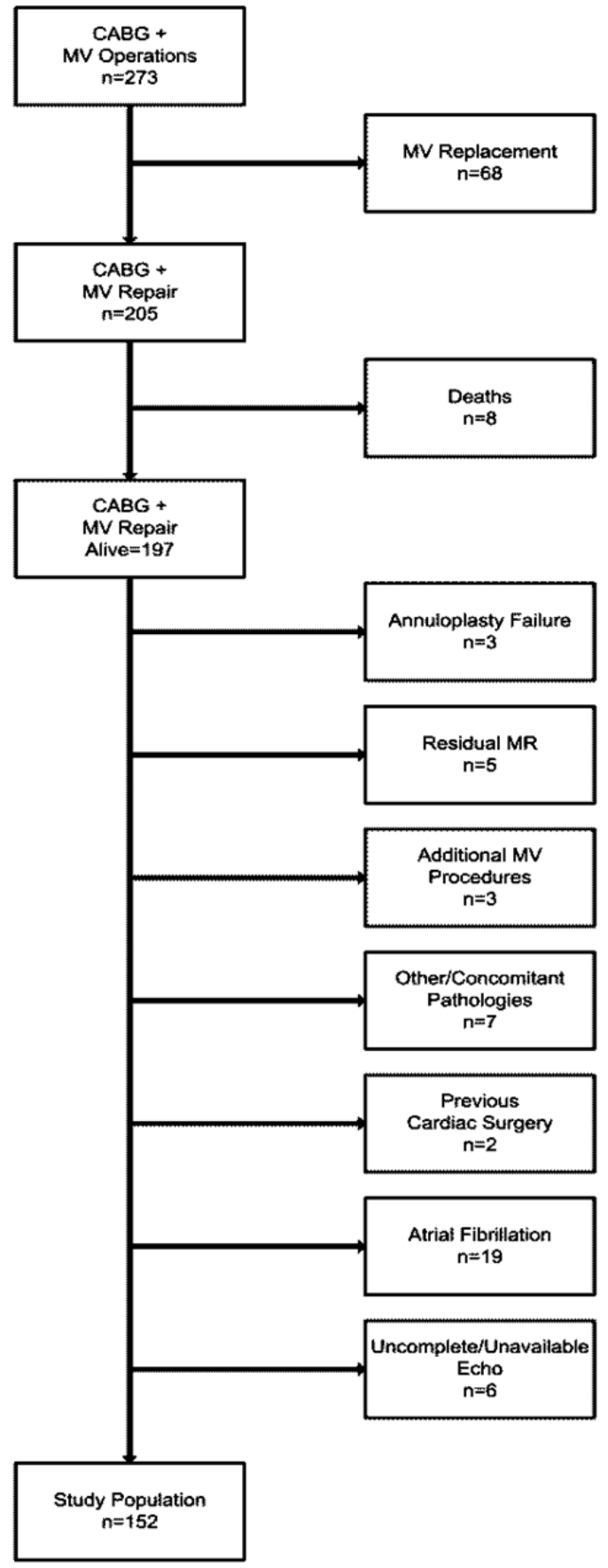

Figure 1. Patient Selection 
Echocardiographic measurements and calculations were carried out as previously reported $^{9}$. The severity of MR was graded on a scale of 1 to 4 according to American Society of Echocardiography Guidelines ${ }^{11}$. In patients with no/trivial MR by color Doppler, RV and RF were used as calculated and EROA was assumed as null. Left ventricular (LV) volumes and LV ejection fraction were assessed using the bi-apical Simpson disk method ${ }^{12}$. Sphericity indexes were obtained at end-diastole and end-systole as the volume of the left ventricle divided by the volume of a sphere with a diameter equal to the longest axis of the left ventricle measured in the apical view ${ }^{13}$. The wall motion score index (WMSI) was calculated according to a 17-segment model ${ }^{14}$. The WMSI of the basal and mid-posterior and inferior segments for the posteromedial papillary muscle (PMPM- WMSI) and basal and mid-lateral and anterior segments for the anterolateral papillary muscle (ALPM-WMSI) were also calculated ${ }^{14}$. The myocardial performance index (MPI) was measured using the method described by Tei and colleagues ${ }^{15}$.

\section{Mitral valve configuration}

Mitral valve configuration was assessed in mid-systole using the parasternal long-axis and four-chamber views (Figure $2 \mathrm{~A}$ ). The AML tethering angle $\alpha$ (between the annular plane and the $A M L$ ) and the posterior mitral leaflet tethering angle $\beta$ (between the annular plane and the PML), were directly measured with a specific software (Philips DICOM Viewer; Philips Medical System). The ratio of $\alpha$ to $\beta$ was a quantitative measurement of tethering: the closer the ratio was to 1 , the more symmetric was the tethering (Figure $2 \mathrm{~B}, \mathrm{C}$ ).

The tenting area (TA) was measured by the area enclosed between the annular plane and the mitral leaflets from the parasternal long-axis view at mid-systole. The coaptation height $(\mathrm{CH})$ was measured as the perpendicular distance between the coaptation point of the mitral leaflets and the line connecting the annular hinge points in the long-axis view at end-systole. The coaptation length (CL) was measured as the length of apposition of the anterior mitral leaflet (AML) and posterior mitral leaflet (PML). The coaptation distance (CD) was measured (along the annular plane) from the anterior leaflet attachment to the point of coaptation. 

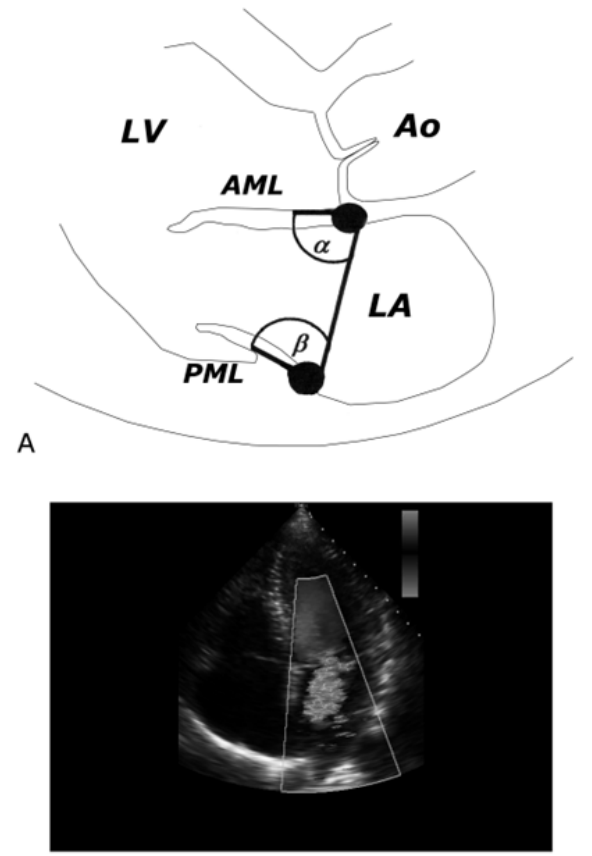

B

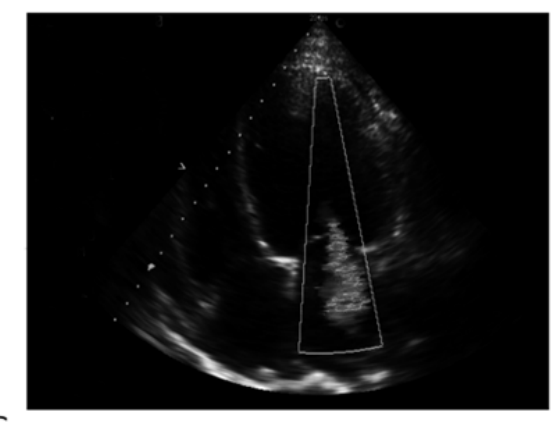

Figure 2. Measurement of tethering angles. A) Tethering of the anterior mitral leaflet (AML) was assessed by measuring the angle $\alpha$ between the annular plane and AML. B) Tethering of the posterior mitral leaflet (PML) was assessed by measuring the angle $\beta$ between the annular plane and the PML. C) Patient belonging to the symmetric group with central regurgitant jet. D) Patient belonging to the asymmetric group with eccentric regurgitant jet.

Abbreviations: LA: Left atrium; LV: Left ventricle; AO: Aorta.

C

\section{Two-dimensional speckle-tracking echocardiography}

The assessment of longitudinal peak systolic strain was performed by applying 2D speckle-tracking imaging to the apical two- and four-chamber views of the LV. The LV was divided into six segments in each apical view and the global longitudinal strain (GLS) was obtained in each view ${ }^{7}$. The peak global longitudinal strain $\left(G-L_{\text {peak }}\right)$ was the mean of the peak negative values on the two G-LS curves (Figure $3 \mathrm{~A}$ ). The assessment of papillary muscle dyssynchrony (DYS-PAP) was performed by applying 2D speckletracking imaging to the apical four-chamber view for anterolateral papillary muscle and to the apical long-axis view for posteromedial papillary muscle ${ }^{7}$. 
From an end-systolic single frame, the edge of each papillary muscle was detected on the endocardial cavity and an automated tracking algorithm (free-trace method, Tomtec, Tomtec Imaging system, Unterschleißheim, Germany) followed the papillary muscle from this single frame throughout the cardiac cycle. The value of peak systolic longitudinal strain for each papillary muscle was then determined as anterolateral papillary muscle longitudinal strain (ALPM-LS) and posteromedial papillary muscle longitudinal strain (PMPM-LS). The beginning of the QRS complex was used as the reference point and the time to peak (TTP) systolic longitudinal strain was quantified for each papillary muscle. For the assessment of DYS-PAP, the absolute difference in TTP between anterolateral and posteromedial papillary a muscle was calculated (Figure $3 \mathrm{~B})$.

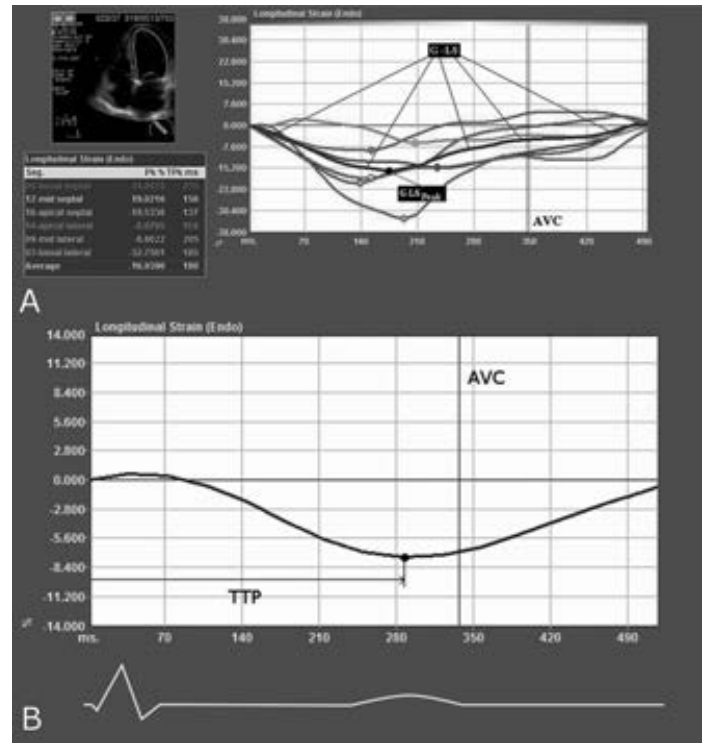

Figure 3. A) Representative example of measuring Global Longitudinal Strain (G-LS) and Global Longitudinal Strain peak $\left(G-L_{\text {peak }}\right)$ in apical 4-chamber view. B) Representative example of measuring papillary muscle dyssynchrony as difference in time to peak (TTP) between anterolateral and posteromedial papillary muscles.

Abbreviations: AVC: Aortic valve closure.

\section{Patient classification}

Patients were divided on the basis of the preoperative anterior/posterior tethering angle ratio $\alpha / \beta$. A cut-off value of 0.76 was chosen based on our previous experience ${ }^{9}$. There were 73 patients with symmetric (Group 1 , mean $\alpha / \beta=0.81 \pm 0.6$ ) and 79 with asymmetric preoperative tethering pattern (Group 2, mean $\alpha / \beta=0.66 \pm 0.4$ ). Patient 
characteristics are summarized in Table 1 . No difference was found in preoperative and operative variables between groups.

\section{Statistical analysis}

Data were analyzed with the use of statistical software (SPSS for Windows, version 12.0; SPSS Inc., Chicago, III). Normality of continuous data was analyzed with the Kolmogorov-Smirnov test.

Data were compared for statistical significance using a t test, Mann-Whitney rank sum test, $\chi 2$ test or Fisher exact test as appropriate. Multiple comparisons were carried out by ANOVA and Kruskal-Wallis tests with Tukey and Dunn post-hoc tests.

Correlations between DYS-PAP and other variables were tested by Pearson's correlation. We assessed, on the entire patient population, univariate regression with postoperative DYS-PAP and regurgitant volume as a dependent variables and other preoperative echocardiographic parameters (end diastolic diameter [EDD], end systolic diameter [ESD], end diastolic volume Index [EDVI], end systolic volume index [ESVI], left ventricular ejection fraction [LVEF], systolic sphericity index [SSI], diastolic sphericity index [DSI], WMSI, MPI, TA, EROA, RV, RF, CH, CL, CD, AML tethering angle $\alpha, P M L$ tethering angle $\beta, \alpha / \beta$ ratio, PMPM-WMSI, ALPM-WMSI) as independent variables. Variables reaching statistical significance or borderline $(p \leq 0.1)$ were introduced in multivariable analysis. Multivariable logistic regression with the forward stepwise method was performed to identify preoperative echocardiographic predictors of significant post-repair DYS-PAP, defined as DYS-PAP $>30 \mathrm{~ms}^{7}$ and significant post-repair recurrent $M R$ defined as $M R \geq$ moderate in patients with no residual MR at discharge. The goodness of fit of the final logistic regression models was assessed with the Hosmer-Lemeshow (H-M) statistic and predictive accuracy was assessed by the concordance index $(\mathrm{c})$. The logistic regression model proved to be reliable $(\mathrm{H}-\mathrm{M}=0.7$ and $\mathrm{H}-$ $\mathrm{M}=0.83$ in the two models, respectively) and accurate ( $c=0.84$ and $c=0.88$ in the two models, respectively). Optimal cut-off values were determined by receiver operating characteristic curve (ROC) as the rounding cut-off that gives the maximum sum of sensitivity and specificity. This value should be the shoulders at the top left of the ROC curve. Results were validated using the bootstrap method (1000 iterations). A p value $<0.05$ was considered statistically significant. 
Table 1. Clinical Characteristics $(n=152)$

\begin{tabular}{|c|c|c|c|}
\hline & $\begin{array}{c}\text { Symmetric } \\
(n=73)\end{array}$ & $\begin{array}{l}\text { Asymmetric } \\
(n=79)\end{array}$ & $\mathrm{p}$ \\
\hline Age, y & $64.4 \pm 6$ & $67.4 \pm 7$ & 0.74 \\
\hline Gender M/F & $47 / 26(64.3 / 35.6)$ & $51 / 28(64.5 / 35.4)$ & 0.90 \\
\hline NYHA class & $3[3-4]$ & $3[3-4]$ & $>0.9$ \\
\hline CCS angina class & $2[1-3]$ & $2[1-3]$ & $>0.9$ \\
\hline \multicolumn{4}{|l|}{ Euroscore } \\
\hline Additive & $8.1[6-8]$ & $7.9[6-8]$ & 0.84 \\
\hline Logistic & 15.3 [11-19] & $14.9[10-18]$ & 0.67 \\
\hline Hypertension & $30(41.1)$ & $29(36.7)$ & 0.53 \\
\hline Diabetes & $21(28.7)$ & $23(29.1)$ & 0.86 \\
\hline COPD & $10(13.7)$ & $11(13.9)$ & 0.93 \\
\hline Chronic renal disease & $12(16.4)$ & $13(16.4)$ & $>0.9$ \\
\hline Cerebral vascular disease & $9(12.3)$ & $10(12.6)$ & 0.88 \\
\hline Peripheral vascular disease & $3(4.1)$ & $6(7.6)$ & 0.07 \\
\hline Familiar history & $37(50.7)$ & $36(45.5)$ & 0.12 \\
\hline Preoperative IABP & $5(6.8)$ & $3(3.8)$ & 0.06 \\
\hline \multicolumn{4}{|l|}{ Myocardial infarction } \\
\hline$>3$ months & $57(78.1)$ & $59(74.7)$ & 0.84 \\
\hline$\leq 3$ months & $16(21.9)$ & $20(25.3)$ & 0.62 \\
\hline Inferior/Posterior & $31(42.4)$ & $44(55.7)$ & 0.57 \\
\hline Anterior/Septal & $8(10.9)$ & $7(8.8)$ & 0.50 \\
\hline Lateral & $9(12.3)$ & $6(7.6)$ & 0.07 \\
\hline Combined & $25(34.2)$ & $22(27.8)$ & 0.09 \\
\hline Coronary vessel disease & $2[1-3]$ & $2[1-3]$ & $>0.9$ \\
\hline Left Main & $14(19.2)$ & $13(16.4)$ & 0.20 \\
\hline \multicolumn{4}{|l|}{ Medications } \\
\hline ACEI/ARB & $62(84.9)$ & $65(82.3)$ & 0.87 \\
\hline$\beta$-adrenergic blockers & $41(56.1)$ & $44(55.7)$ & 0.64 \\
\hline Long-acting nitrates & $33(45.2)$ & $35(44.3)$ & 0.89 \\
\hline Diuretics & $63(86.3)$ & $68(86.1)$ & 0.9 \\
\hline \multicolumn{4}{|l|}{ Surgery } \\
\hline CPB time (min) & 108 [99-125] & 102 [94-115] & 0.08 \\
\hline CCL time (min) & $96[77-104]$ & $83[70-97]$ & 0.12 \\
\hline Mitral Ring size (mm) & $28[26-30]$ & $28[26-30]$ & 0.90 \\
\hline \multicolumn{4}{|l|}{ CABG } \\
\hline Anastomoses/patient & $2[1-3]$ & $2[1-3]$ & $>0.9$ \\
\hline Arterial graft/patient & $1[1-2]$ & $1[1-2]$ & $>0.9$ \\
\hline
\end{tabular}

Normally- distributed variables are presented as mean \pm standard deviation; Discrete variables are presented as percentages. Non-normally distributed variables are presented as median [Interquartile range].

Abbreviations: $\mathrm{M} / \mathrm{F}=$ Male/Female; NYHA = New York Heart Association; $\mathrm{CCS}=$ Canadian Cardiovascular Society; COPD = Chronic obstructive pulmonary disease; IABP = Intra-aortic balloon pump; $A C E I=$ angiotensin-converting enzyme inhibitor; $\mathrm{ARB}=$ angiotensin receptor blocker; $\mathrm{CPB}=$ Cardiopulmonary bypass; $\mathrm{CCL}=$ (Aortic) Cross-clamp; CABG = Coronary artery bypass grafting. 


\section{Results}

\section{Echocardiographic results and recurrence of mitral regurgitation}

At follow-up, recurrent MR occurred in $67.1 \%(n=49)$ in Group 1 vs. $3.8 \%(n=3)$ in Group2 $(p<0.001)$. In the symmetrical Group, at baseline, EDD $(p=0.005)$, ESD $(p=0.007)$, ESVI $(p=0.02)$, EDVI $(p<0.001)$, and sphericity Indexes (both, $p<0.001)$ were higher whereas LVEF was lower $(p<0.001)$. Furthermore, in this Group, TA $(p<0.001)$, and $\mathrm{CH}(\mathrm{p}=0.04)$ were preoperatively higher and $\mathrm{CL}(\mathrm{p}=0.01)$ was shorter. In addition, patients with symmetrical tethering had higher tethering angle $\alpha(p<0.001)$, lower tethering angle $\beta(p=0.001)$ and larger ALPM-WMSI $(p=0.03)$. Contrastingly, the PMPM-WMSI was higher in Group $2(p=0.03)$. At late follow up the tethering angle $\alpha$ diminished significantly from baseline in both Groups $(p<0.001)$ remaining still higher in the symmetrical Group ( $p<0.001$ vs. asymmetrical) whereas the tethering angle $\beta$ increased in both Groups ( $p<0.001$ vs. baseline) and was comparable at follow up between groups $(p=0.4)$. Furthermore, at the latest control, the asymmetrical group had smaller LV dimensions $(p<0.001)$, LV volumes $(p<0.001)$ and sphericity indexes $(p<0.001)$ and lower global WMSI $(p=0.007)$. Finally, in both Groups, PMPM-WMSI increased significantly at follow up ( $p=0.005$ and $p=0.03$ vs. baseline, in symmetric and asymmetric respectively) whereas ALPM-WMSI remained constant ( $p>0.9$ and $p=0.8$ vs. baseline, in symmetric and asymmetric respectively). All echocardiographic data are shown in Table 2.

Table 2. Echocardiographic Results ( $n=152)$

\begin{tabular}{|c|c|c|c|c|c|c|}
\hline & \multicolumn{3}{|c|}{$\begin{array}{l}\text { Symmetric tethering pattern } \\
\qquad(n=73)\end{array}$} & \multicolumn{3}{|c|}{$\begin{array}{l}\text { Asymmetric tethering pattern } \\
\qquad(\mathrm{n}=79)\end{array}$} \\
\hline & Baseline & Discharge & F-UP & Baseline & Discharge & F-UP \\
\hline \multicolumn{7}{|l|}{$\overline{M R}$} \\
\hline \multicolumn{7}{|c|}{ Direction of regurgitant jet } \\
\hline Central & $45(61.7)^{\ddagger}$ & - & $1(2.0)$ & $7(8.8)$ & - & - \\
\hline Anterior & $6(8.2)$ & - & $1(2.0)$ & $9(11.4)$ & - & - \\
\hline Posterior & $9(12.3)^{\ddagger}$ & - & $45(91.8)^{\ddagger}$ & $49(62.1)$ & - & $2(66.7)$ \\
\hline Complex & $13(17.8)$ & - & $2(4.2)^{\ddagger}$ & $14(17.7)$ & - & $1(33.3)$ \\
\hline \multicolumn{7}{|c|}{ Quantitative data } \\
\hline $\mathrm{TA}\left(\mathrm{cm}^{2}\right)$ & $4.1 \pm 1.0^{\ddagger}$ & $2.1 \pm 0.2^{*}$ & $2.7 \pm 0.9^{+}$ & $3.2 \pm 1.0$ & $1.8 \pm 0.6^{*}$ & $1.8 \pm 0.3$ \\
\hline EROA $\left(\mathrm{mm}^{2}\right)$ & $37 \pm 12$ & - & $29 \pm 12^{\ddagger}$ & $34 \pm 9$ & - & - \\
\hline $\operatorname{RF}(\%)$ & $48 \pm 13$ & - & $29 \pm 12^{\ddagger}$ & $44 \pm 15$ & - & - \\
\hline $\mathrm{RV}(\mathrm{ml})$ & $56 \pm 14$ & - & $35 \pm 13^{\ddagger}$ & $60 \pm 15$ & - & - \\
\hline \multicolumn{7}{|c|}{ Mitral leaflets tethering } \\
\hline$\alpha\left(^{\circ}\right)$ & $42.8 \pm 7^{\ddagger}$ & $36.8 \pm 7^{* \ddagger}$ & $34.9 \pm 9^{* \ddagger}$ & $33.8 \pm 4$ & $24.8 \pm 6^{*}$ & $23.9 \pm 5^{*}$ \\
\hline$\beta\left({ }^{\circ}\right)$ & $44.8 \pm 12^{\ddagger}$ & $61.9 \pm 11^{*}$ & $75.4 \pm 13^{*+}$ & $50.9 \pm 9$ & $60.0 \pm 11^{*}$ & $77.6 \pm 13^{*+}$ \\
\hline $\mathrm{CH}(\mathrm{cm})$ & $1.3 \pm 0.3^{\ddagger}$ & $0.8 \pm 0.1^{*}$ & $1.8 \pm 0.3^{* \dagger \ddagger}$ & $1.2 \pm 0.3$ & $0.8 \pm 0.2^{*}$ & $0.8 \pm 0.2^{*}$ \\
\hline $\mathrm{CL}(\mathrm{cm})$ & $3.1 \pm 0.1^{\ddagger}$ & $8.3 \pm 0.3^{*}$ & $2.6 \pm 0.2^{\dagger \ddagger}$ & $4.3 \pm 0.7$ & $8.6 \pm 0.5^{*}$ & $8.7 \pm 0.3^{*}$ \\
\hline $\mathrm{CD}(\mathrm{cm})$ & $3.6 . \pm 0.5$ & $3.3 \pm 0.6^{*}$ & $3.7 \pm 0.7^{\dagger}$ & $3.5 . \pm 0.8$ & $3.1 \pm 0.6^{*}$ & $3.1 \pm 0.5$ \\
\hline
\end{tabular}




\begin{tabular}{|c|c|c|c|c|c|c|}
\hline & \multicolumn{3}{|c|}{$\begin{array}{l}\text { Symmetric tethering pattern } \\
\qquad(\mathrm{n}=73)\end{array}$} & \multicolumn{3}{|c|}{$\begin{array}{l}\text { Asymmetric tethering pattern } \\
\qquad(\mathrm{n}=79)\end{array}$} \\
\hline & Baseline & Discharge & F-UP & Baseline & Discharge & F-UP \\
\hline \multicolumn{7}{|c|}{ Global LV remodeling } \\
\hline $\mathrm{EDD}(\mathrm{mm})$ & $66 \pm 7^{\ddagger}$ & $52 \pm 8^{*}$ & $57 \pm 6^{* \neq \ddagger}$ & $59 \pm 7$ & $53 \pm 7^{*}$ & $41 \pm 4^{+}$ \\
\hline $\mathrm{ESD}(\mathrm{mm})$ & $56 \pm 5^{\ddagger}$ & $44 \pm 7^{*}$ & $48 \pm 5^{* \pm \ddagger}$ & $49 \pm 8$ & $44 \pm 7^{*}$ & $34 \pm 3^{+}$ \\
\hline $\operatorname{EDVI}\left(\mathrm{mL} / \mathrm{m}^{2}\right)$ & $112 \pm 16^{\ddagger}$ & $87 \pm 13^{* \ddagger}$ & $110 \pm 15^{\dagger \ddagger}$ & $105 \pm 11$ & $93 \pm 10^{*}$ & $79 \pm 7^{+}$ \\
\hline ESVI $\left(\mathrm{mL} / \mathrm{m}^{2}\right)$ & $74 \pm 9^{\ddagger}$ & $59 \pm 7^{* \pm}$ & $72 \pm 5^{\dagger \ddagger}$ & $59 \pm 8$ & $49 \pm 7^{*}$ & $42 \pm 3^{+}$ \\
\hline LVEF (\%) & $33 \pm 9^{\ddagger}$ & $32 \pm 7^{\ddagger}$ & $34 \pm 5^{\ddagger}$ & $43 \pm 9$ & $47 \pm 9^{*}$ & $46 \pm 7$ \\
\hline SSI & $0.82 \pm 0.1^{\ddagger}$ & $0.76 \pm 0.1^{* \ddagger}$ & $0.73 \pm 0.2^{* \pm}$ & $0.62 \pm 0.1$ & $0.43 \pm 0.1^{*}$ & $0.37 \pm 0.1^{\dagger}$ \\
\hline DSI & $0.87 \pm 0.1^{\ddagger}$ & $0.83 \pm 0.1^{\ddagger}$ & $0.84 \pm 0.1^{\ddagger}$ & $0.75 \pm 0.1$ & $0.51 \pm 0.1^{*}$ & $0.41 \pm 0.1^{\dagger}$ \\
\hline WMSI & $1.4 \pm 0.6^{\ddagger}$ & $1.3 \pm 0.3^{\ddagger}$ & $1.7 \pm 0.3^{\ddagger}$ & $1.2 \pm 0.3$ & $1.0 \pm 0.2^{*}$ & $1.1 \pm 0.3^{\dagger}$ \\
\hline MPI & $0.92 \pm 0.1^{\ddagger}$ & $0.90 \pm 0.1^{\ddagger}$ & $0.93 \pm 0.1^{\ddagger}$ & $0.66 \pm 0.1$ & $0.57 \pm 0.1^{*}$ & $0.50 \pm 0.1^{+}$ \\
\hline \multicolumn{7}{|c|}{ Local LV remodeling } \\
\hline PMPM-WMSI & $2.1 \pm 0.3^{\ddagger}$ & $2.2 \pm 0.3^{* \ddagger}$ & $2.5 \pm 0.3^{*+\ddagger}$ & $2.5 \pm 0.4$ & $2.3 \pm 0.3^{*}$ & $2.7 \pm 0.31^{*+}$ \\
\hline ALPM-WMSI & $1.5 \pm 0.3^{\ddagger}$ & $1.4 \pm 0.3^{\ddagger}$ & $1.5 \pm 0.3^{\ddagger}$ & $1.1 \pm 0.1$ & $0.9 \pm 0.1^{*}$ & $1.0 \pm 0.1$ \\
\hline
\end{tabular}

Normally-distributed variables are presented as mean \pm standard deviation. Discrete variables are presented as percentages. Non-normally distributed variables are presented as median [Interquartile range]. ${ }^{* \dagger}$ Significance vs. baseline and discharge, respectively; ${ }^{\ddagger}$ Significance vs. the asymmetrical Group.

Abbreviations: TA = Tenting area; $\mathrm{EROA}=$ Effective regurgitant orifice area; $\mathrm{RF}=$ Regurgitant Fraction; RV = Regurgitant Volume; $\alpha=$ Anterior mitral leaflet tethering angle; $\beta=$ posterior mitral leaflet tethering angle; $\mathrm{CH}=$ coaptation height; $\mathrm{CL}=$ Coaptation Length; $\mathrm{CD}=$ Coaptation Distance; $\mathrm{EDD}=$ End Diastolic Diameter; ESD = End Systolic Diameter; EDVI = End Diastolic Volume Index; ESVI = End Systolic Volume Index; LVEF = Left Ventricular Ejection Fraction; SSI = Systolic Sphericity Index; DSI = Diastolic Sphericity Index; MPI = Myocardial Performance Index; WMSI = Wall Motion Score Index; PMPM = Posteromedial papillary Muscle; ALPM $=$ Anterolateral papillary muscle.

\section{Two-dimensional speckle-tracking analysis}

Comparing both Groups at baseline, patients in Group 1 had higher DYS-PAP $(57.7 \pm$ $5.3 \mathrm{~ms}$ vs. $29.8 \pm 2.4 \mathrm{~ms}, \mathrm{p}<0.001)$ and worse values of longitudinal strain (LS) in ALPM (ALPM-LS, $-6.8 \pm 0.4 \%$ vs. $-11.9 \pm 0.5 \%, p<0.001$ ), PMPM (PMPM-LS, $-5.5 \pm 0.3 \%$ vs.$10.9 \pm 0.3 \%, p<0.001)$ and global LS (G-LS, $-0.9 \pm 0.2 \%$ vs. $-7.0 \pm 0.7 \%$ vs. $p<0.001)$. At follow up, in patients in Group 1, ALPM-LS (-6.9 $\pm 0.2 \%, p>0.9$ vs. baseline) PMPM-LS ($5.0 \pm 0.5 \%, p=0.77$ vs. baseline) and $\mathrm{G}_{-\mathrm{LS}}$ peak $(0.9 \pm 0.2 \%, \mathrm{p}>0.9$ vs. baseline) did not change whereas DYS-PAP significantly worsened (78.1 $\pm 8.8 \mathrm{~ms}, \mathrm{p}<0.001$ vs. baseline [Figure $4 \mathrm{~A}-\mathrm{B}$, Figure 5]). In contrast, in patients in Group 2, ALPM-LS $(-19.9 \pm 0.9 \%$, $\mathrm{p}<0.001$ vs. baseline), PMPM-LS (-16.7 $\pm 0.8 \%, \mathrm{p}<0.001$ vs. baseline) and $\mathrm{G}-\mathrm{LS}_{\text {peak }}(-15.5$ $\pm 0.8 \%, p<0.001$ vs. baseline) and DYS-PAP (10.6 $\pm 3.0 \mathrm{~ms}, \mathrm{p}<0.001$ vs. baseline) improved (Figure $4 \mathrm{C}-\mathrm{D}$, Figure 5). At follow up, 68 patients (93.1\%) belonging to the symmetrical Group and 2 (2.5\%) with preoperative asymmetrical tethering had a DYSPAP >30 ms ( $<<0.001)$. In the latter Group, 13 patients $(16.5 \%)$ has DYS-PAP $15-30 \mathrm{~ms}$ and $64(81.0 \%)<15 \mathrm{~ms}$. 

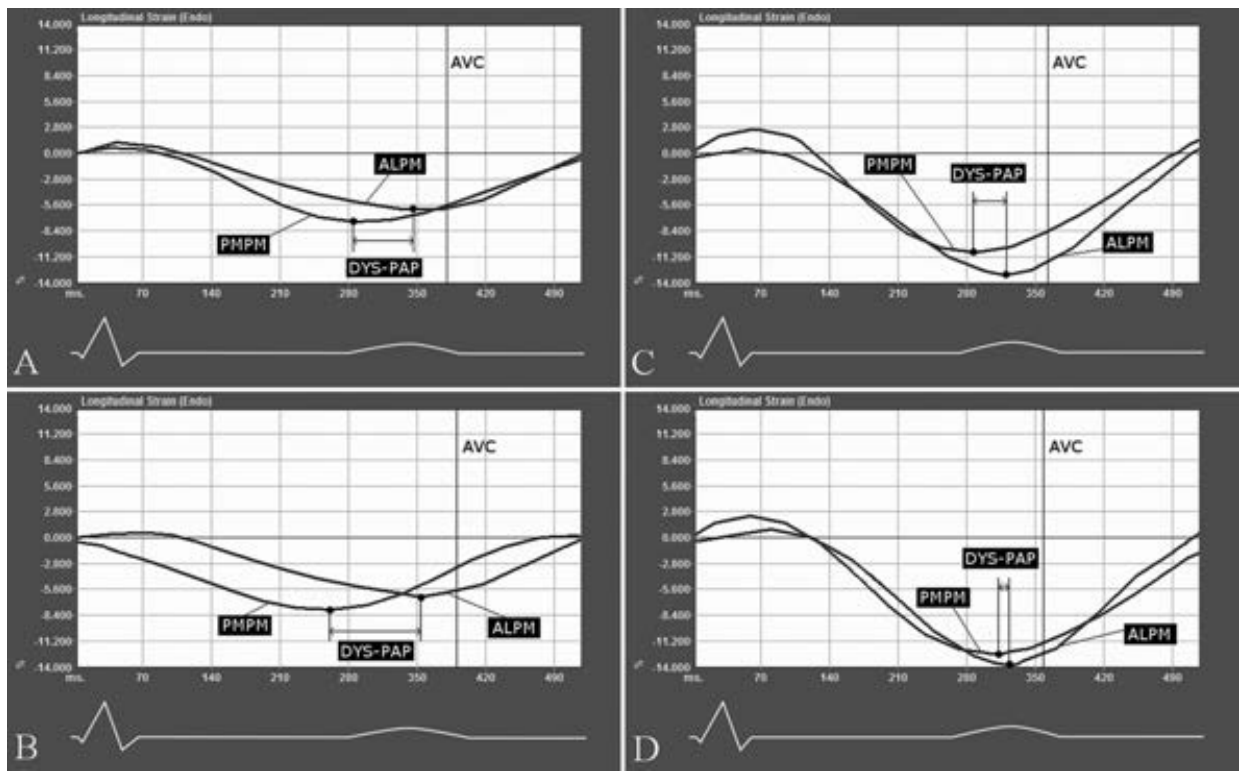

Figure 4. A) Preoperative papillary muscle dyssynchrony (DYS-PAP) in a patient with symmetrical tethering (60.3 $\mathrm{ms})$. B) Postoperative DYS-PAP in the same patient (95.2 ms) as in A. C) Preoperative DYS-PAP in a patient with asymmetrical tethering (35.5 ms). D) Postoperative DYS-PAP in the same patient (11.9 ms) as in C.

Abbreviations: AVC: Aortic valve closure; ALPM: Anterolateral papillary muscle; PMPM: posteromedial papillary muscle; DYS-PAP: papillary muscle dyssynchrony.
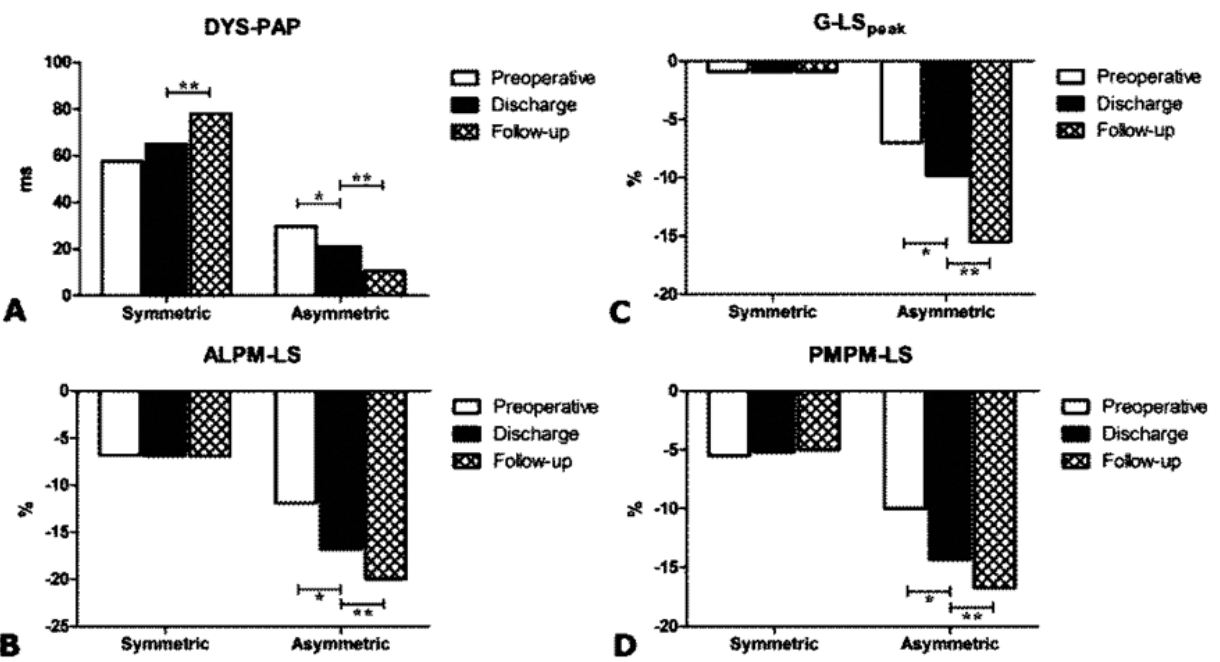

Figure 5. A) Papillary muscle dyssynchrony (DYS-PAP) at baseline, discharge and follow-up in the two Groups. B) Anterolateral papillary muscle longitudinal strain (ALPM-LS) at baseline, discharge and follow-up in the two Groups. C) Global longitudinal strain (GL-S) at baseline, discharge and follow-up in the two Groups.. D) Posteromedial papillary muscle longitudinal strain (PMPM-LS) at baseline, discharge and follow-up in the two Groups. *Significance vs. baseline. ** Significance vs. discharge. 


\section{Echocardiographic predictors of postoperative DYS-PAP}

There was a strong correlation between $\alpha / \beta$ and baseline DYS-PAP $(r=0.95, p<0.001)$. Furthermore, preoperative $\alpha / \beta$, $\alpha$ tethering angle, SSI, ESVI, MPI, EDVI , EDD, ESD and WMSI correlated strongly with postoperative DYS-PAP (all, $p<0.001$; Table 3). DSI $(p=0.004)$ had moderate correlation. Other preoperative variables did not correlate significantly with postoperative DYS-PAP (Table 3 ).

At multivariable analysis (Figure 6$) \alpha / \beta(p<0.001)$, tethering angle $\alpha(p<0.007)$, SSI $(p=0.01)$, ESVI $(p=0.04)$ and MPI $(p=0.04)$ were predictors of significant postoperative DYS-PAP.

On ROC curves, the best preoperative predictor of significant postoperative DYSPAP was $\alpha / \beta$ with a cut-off of $\geq 0.76$ (sensitivity $100 \%$, specificity $90 \%$, area under curve [AUC] 0.98 (95\% Cl 0.96-1).

Anterior tethering angle $\alpha$ had $90 \%$ sensitivity and $82 \%$ specificity with an optimal cut-off of $\geq 39.5^{\circ}$ (AUC 0.88 [95\% Cl: $0.77-0.93$ ], SSI with a cut-off of $\geq 0.7$ had $83 \%$ sensitivity and $75 \%$ specificity (AUC 0.78 [95\% Cl: $0.70-0.87$ ], MPI with a cut-off $\geq 0.88$ had $80 \%$ sensitivity and $70 \%$ specificity (AUC 0.74 [95\% Cl: $0.68-0.84$ ] and ESVI with a cut-off $\geq 60 \mathrm{~mL} / \mathrm{m}^{2}$ had $73 \%$ sensitivity and $66 \%$ specificity (AUC 0.70 [95\% Cl: 0.64 0.80].

\section{Echocardiographic predictors of recurrent mitral regurgitation}

There was a significant correlation between recurrent MR and DYS-PAP $(r=0.87$, $p<0.001), \alpha$ angle $(r=0.85, p<0.001)$ and $\alpha / \beta \quad(r=0.86, p<0.001)$ and SSI $(r=0.82$, $\mathrm{p}<0.001)$. There was no significant correlation between MR recurrence and other echocardiographic parameters.

Logistic regression analysis revealed that DYS-PAP (OR: $5.0,95 \% \mathrm{Cl}: 3.6-7.3$, $\mathrm{p}<0.001$ ), AML tethering angle $\alpha$ (OR: $4.4,95 \% \mathrm{Cl}: 2.7-6.3, \mathrm{p}<0.001$ ), tethering symmetry (OR: 4.0, 95\% Cl: 2.7-5.2, p<0.001) and systolic sphericity Index (OR: 4.3, 95\% Cl: $2.8-5.6, p<0.001$ ) were predictors of recurrent MR. A DYS-PAP value $\geq 58$ ms predicted recurrence of MR with $95 \%$ sensitivity and $88 \%$ specificity (AUC 0.92, 95\% Cl: $0.7-1$, $\mathrm{p}<0.001)$. Anterior tethering angle $\alpha$ had $90 \%$ sensitivity and $83 \%$ specificity with an optimal cut-off of $\geq 39.5^{\circ}$ (AUC $0.84,95 \% \mathrm{Cl} 0.70-0.91, p<0.001$ ); $\alpha / \beta$ with a cut-off of $\geq$ 0.76 had $87 \%$ sensitivity and $80 \%$ specificity (AUC $0.82,95 \% \mathrm{Cl} 0.78-0.87, \mathrm{p}<0.001$ ). Finally, SSI $\geq 0.72$ had $88 \%$ sensitivity and $76 \%$ specificity with an with an area under curve of 0.77 ( $95 \% \mathrm{Cl}: 0.64-0.88)$ in predicting MR recurrence. 
Table 3. Univariate Linear Regression Analyses for Echocardiographic Factors Predicting Post-repair DYS-PAP.

\begin{tabular}{lll}
\hline & $r$ & $p$ \\
\hline End diastolic diameter & 0.324 & $<0.001$ \\
End systolic diameter & 0.391 & $<0.001$ \\
End systolic volume index & 0.527 & $<0.001$ \\
End diastolic volume index & 0.373 & $<0.001$ \\
Left Ventricular ejection fraction & -0.07 & 0.45 \\
Systolic sphericity index & 0.761 & $<0.001$ \\
Diastolic sphericity index & 0.273 & 0.004 \\
Wall motion score index & 0.331 & $<0.001$ \\
Myocardial performance index & 0.477 & $<0.001$ \\
Tenting area & 0.110 & 0.132 \\
Effective regurgitant orifice area & 0.116 & 0.167 \\
Regurgitant fraction & 0.054 & 0.398 \\
Regurgitant volume & 0.066 & 0.401 \\
Coaptation height & 0.045 & 0.605 \\
Coaptation length & 0.040 & 0.613 \\
$\alpha$ (anterior mitral leaflet tethering angle) & 0.883 & $<0.001$ \\
$\beta$ (posterior mitral leaflet tethering angle) & 0.015 & 0.884 \\
$\alpha / \beta$ (tethering pattern) & 0.90 & $<0.001$ \\
Coaptation distance & 0.070 & 0.408 \\
Wall motion score index at posteromedial papillary muscle & 0.049 & 0.603 \\
Wall motion score index at anterolateral papillary muscle & 0.036 & 0.683 \\
\hline & &
\end{tabular}

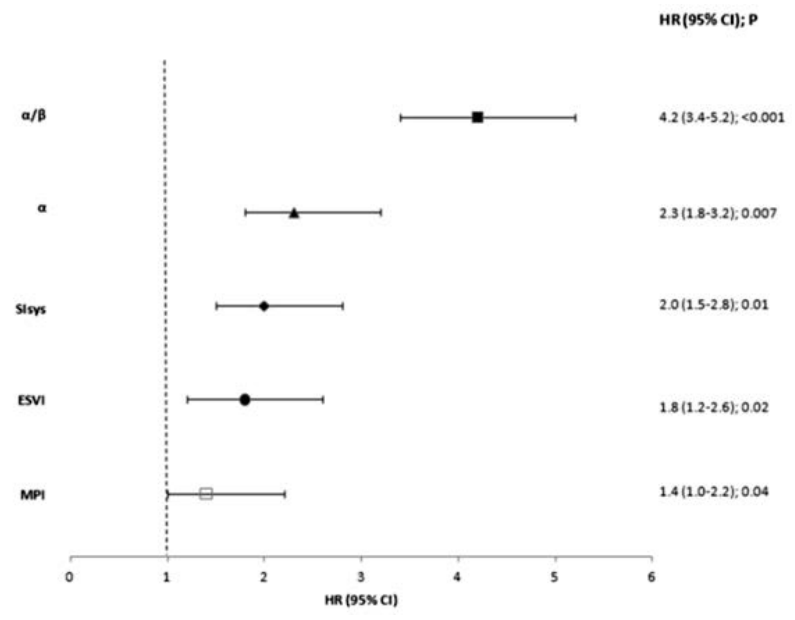

Figure 6. Multivariable predictors of postoperative papillary muscle dyssynchrony.

Abbreviations: $\alpha$ : anterior mitral leaflet tethering angle; $\beta$ : posterior mitral leaflet tethering angle; $\mathrm{SI}_{\text {sys }}$ : Systolic sphericity Index; ESVI: End Systolic Volume Index; MPI: Myocardial Performance Index. 


\section{Discussion}

Chronic ischemic mitral regurgitation (CIMR) occurs despite a structurally normal mitral valve as a consequence of LV dysfunction ${ }^{16}$. LV distortion and remodeling after $\mathrm{MI}$ displaces papillary muscles from the mitral annulus ${ }^{17}$ and this displacement puts excessive tension on the chordae resulting both in the tethering of the mitral leaflets and in restricting their coaptation during systole ${ }^{16}$. Agricola and coworkers ${ }^{18}$ first recognized, among patients with CIMR due to restricted motion, two groups according to the tethering pattern: the asymmetrical group with predominant posterior tethering of both leaflets and the symmetrical one with predominant apical tethering of both leaflets. Since each papillary muscle supplies chordae to both leaflets, consequentially a posterior displacement of only one papillary muscle invariably exerts traction on both leaflets $^{19}$. Therefore, the different shapes of tethering of the two groups depend on the relationship of the three tethering vectors (posterior, apical and lateral). In the asymmetrical group, the posterior leaflet is simply drawn more posteriorly than apically (more parallel to the posterior wall). This posterior restriction of the posterior leaflet prevents it from reaching its normal, more anteriorly located coaptation point, so that the coaptation point moves posteriorly, creating the asymmetrical tethering shape. In the symmetrical one, there is additional apical, and medio-lateral tethering in addition to the posterior component. The net result of these forces is more apical tenting, with the coaptation point being displaced more apically. In the symmetrical group the regurgitant jet usually has a central origin and direction because the systolic motion of both leaflets is equally affected. In the asymmetrical one, it is the movement of the posterior leaflet to be predominantly compromised causing posterior jet direction ${ }^{18}$. In our recent studies the symmetrical tethering proved to be a strong predictor of recurrent mitral regurgitation ${ }^{20}$ and lack of LV reverse remodeling ${ }^{21}$ after restrictive annuloplasty for CIMR. As a measure of tethering symmetry we employed the ratio between the anterior and posterior tethering angles which represents an indirect measurement of the resultant tethering vector.

Left ventricular dyssynchrony is a frequently observed feature in patients with functional ischemic mitral regurgitation and it may contribute to MR by several mechanisms: 1) Uncoordinated regional LV mechanical activation results in geometrical changes in mitral leaflet attachments and tethering of the mitral leaflets; 2) A positive pressure gradient develops between the left atrium and left ventricle due to improper timing of atrio-ventricular relaxation and contraction cycles, which effects mitral valve closure; 3) LV dyssynchrony decreases LV contraction efficiency and closing forces, thereby impairing mitral valve tenting ${ }^{22}$.

No information exists about the relationship between DYS-PAP and tethering pattern in CIMR patients and whether the persistence of dyssynchronous papillary muscle activation after surgery may be related to a specific leaflet configuration. In this study 
we assessed global ventricular strain and DYS-PAP in CMIR patients, divided on the basis of preoperative tethering pattern into symmetrical and asymmetrical.

We employed two-dimensional speckle-tracking (2D-ST), a no-Doppler-based method which provides angle-independent assessment of multidirectional LV strain and differentiates myocardial segments with active contraction from segments that are passively tethered ${ }^{7}$. To our knowledge this is the first study investigating both LV strain and papillary muscle dyssynchrony in patients with CIMR utilizing 2D-STE analysis.

Our experience demonstrated that, in patients with preoperative asymmetrical tethering, G-LS $S_{\text {peak, }}$ PMPM-LS, ALPM-LS $(p<0.001)$ and DYS-PAP $(p=0.01)$ significantly improved at follow-up. More specifically, $16.5 \%(n=13)$ patients with preoperative asymmetrical pattern had postoperative DYS-PAP comprised of between $15 \mathrm{~ms}$ and 30 $\mathrm{ms}, 81 \%(\mathrm{n}=64)<15 \mathrm{~ms}$ and $2.5 \%(\mathrm{n}=2)>30 \mathrm{~ms}$. In contrast, in patients with preoperative symmetrical pattern, DYS-PAP worsened and 93.1\% (68/73) of these patients had DYS-PAP >30 ms at follow-up. Moreover, symmetrical pattern was the strongest predictor of significant postoperative DYS-PAP. Finally, in accordance with our previous experience ${ }^{6}$, symmetrical tethering pattern resulted in a significantly higher postoperative MR rate $(67.1 \%$ vs. $3.8 \%, p<0.001)$ and a DYS-PAP $\geq 58$, anterior tethering angle $\alpha$ $\geq 39.5^{\circ}, \alpha / \beta \geq 0.76$ and $\mathrm{SSI} \geq 0.72$ were predictors of MR recurrence.

Compared to data published in the literature in no-ischemic patients ${ }^{7}$, we had lower values of DYS-PAP and this might be attributable to the small number of subjects with QRS >120 ms (20.\%) which reflects significant mechanical dyssynchrony not associated with electrical dyssynchrony in the majority of patients in our cohort. This is in accordance with recent data showing that the QRS duration does not adequately reflect LV dyssynchrony which may occur only in $30 \%$ of heart failure patients ${ }^{23}$. Published data show that reduction of ischemia by CABG is associated with resynchronization only in a small percentage of patients with a high degree of preoperative dyssynchrony $^{24}$. Accordingly, in our experience, in patients in Group 1 with significant preoperative DYS-PAP, it did not improve after revascularization.

It has been demonstrated that cardiac resynchronization therapy (CRT) reduces the degree of functional MR both immediately ${ }^{25}$ and in the long -term ${ }^{26}$ and that the improvement in LV dyssynchrony plays an important role in the development of reverse remodeling after $\mathrm{CRT}^{25}$.

Improved coordination of papillary muscle contraction might contribute to ensuring post-repair mitral competence in these patients by re-coordinating the tethering forces on papillary muscles and increasing closing force on the mitral leaflets. Furthermore, it has been demonstrated that the improvement in LV dyssynchrony plays an important role in the development of reverse remodeling after $\mathrm{CRT}^{27}$. Therefore, we might suppose that re-synchronization successfully interrupts the vicious circle of remodeling and turns it into the virtuous circle of reverse remodeling and further resynchronization. Indeed, patients with asymmetrical tethering and without significant 
preoperative dyssynchrony, showed significant reverse remodeling and MV competence at follow-up. In contrast, in patients with symmetrical tethering, DYS-PAP was irreversible; the lack of re-synchronization might have led to significant volume overload which promoted further LV dilatation, continuous remodeling and recurrent MR.

\section{Clinical implications}

The main finding of our study was the correlation of preoperative tethering pattern and DYS-PAP and the evidence that significant dyssynchrony after surgery can be predicted from the configuration of the mitral valve on the preoperative echocardiographic assessment. Onset of LV dyssynchrony is a marker of advanced cardiac dysfunction and resynchronization of LV contractions is associated with reverse remodeling and reduction in MR.

The symmetric pattern might represent an advanced stage of the ischemic disease with dilated-dysfunctional left ventricle and advanced DYS-PAP and it might reflect an irreversible, mechanical LV dyssynchrony. Tethering symmetry may, therefore, represent an easy and immediate tool to identify patients who cannot benefit from UMRA. Thus, an accurate evaluation of tethering pattern before surgery should be incorporated into risk prediction models for unsuccessful MV repair and, in patients with preoperative symmetrical tethering pattern, the risk of performing UMRA must be carefully weighed and concomitant or alternative surgical techniques should be considered ${ }^{9}$ $21,28$.

On the basis of these data and from our previous experiences ${ }^{6,9,20,21}$ we believe that UMRA can be safely employed only in selected patients identified on the basis of clinical and echocardiographic features. However, in our opinion, this selection should include also data on myocardial viability and dyssynchrony. Therefore, UMRA is indicated in patients with echocardiographic asymmetric pattern: 1) in presence of viable myocardium surrounding the scar; 2 ) in absence of papillary muscle dyssynchrony; 3 ) in patients with $\mathrm{AML}<39.5^{\circ} ; 4$ ) in patients with systolic sphericity index $<0.7 ; 5$ ) in absence of advanced diastolic dysfunction.

\section{Study limitations}

The main limitation of the present study is the lack of information on myocardial viability. This was due to its retrospective nature and to the fact that viability was not routinely documented. The presence of viable myocardium with concomitant absence of LV dyssynchrony might be responsible for favorable results in the asymmetrical Groups. This aspect deserves further evaluation.

In addition, as the myocardial movement is basically three-dimensional, thus including rotational movements, two-dimensional speckle tracking may have inherent limitations, especially in patients with dilated LV. Finally, the cut-off $>30 \mathrm{~ms}$ is an accepted 
threshold for patient with dilated non-ischemic cardiomyopathy and it has not been validated for CIMR patients.

\section{Conclusions}

Preoperative tethering symmetry identifies CIMR patients with advanced DYS-PAP who are unlikely to benefit from mitral repair. Further larger studies are necessary to confirm our findings. 


\section{References}

1. Diodato MD, Moon MR, Pasque MK, Barner HB, Moazami N, Lawton JS, Bailey MS, Guthrie TJ, Meyers $B F$, Damiano RJ, Jr. Repair of ischemic mitral regurgitation does not increase mortality or improve longterm survival in patients undergoing coronary artery revascularization: A propensity analysis. The Annals of thoracic surgery. 2004;78:794-799; discussion 794-799

2. Bouma W, van der Horst IC, Wijdh-den Hamer IJ, Erasmus ME, Zijlstra F, Mariani MA, Ebels T. Chronic ischaemic mitral regurgitation. Current treatment results and new mechanism-based surgical approaches. European journal of cardio-thoracic surgery: official journal of the European Association for Cardio-thoracic Surgery. 2010;37:170-185

3. Lee AP, Acker M, Kubo SH, Bolling SF, Park SW, Bruce CJ, Oh JK. Mechanisms of recurrent functional mitral regurgitation after mitral valve repair in nonischemic dilated cardiomyopathy: Importance of distal anterior leaflet tethering. Circulation. 2009;119:2606-2614

4. Kuwahara E, Otsuji Y, Iguro Y, Ueno T, Zhu F, Mizukami N, Kubota K, Nakashiki K, Yuasa T, Yu B, Uemura T, Takasaki K, Miyata M, Hamasaki S, Kisanuki A, Levine RA, Sakata R, Tei C. Mechanism of recurrent/persistent ischemic/functional mitral regurgitation in the chronic phase after surgical annuloplasty: Importance of augmented posterior leaflet tethering. Circulation. 2006;114:1529-1534

5. Magne J, Pibarot P, Dagenais F, Hachicha Z, Dumesnil JG, Senechal M. Preoperative posterior leaflet angle accurately predicts outcome after restrictive mitral valve annuloplasty for ischemic mitral regurgitation. Circulation. 2007;115:782-791

6. Gelsomino S, Van Garsse L, Luca F, Lorusso R, Cheriex E, Rao CM, Caciolli S, Vizzardi E, Crudeli E, Stefano $P$, Gensini GF, Maessen J. Impact of preoperative anterior leaflet tethering on the recurrence of ischemic mitral regurgitation and the lack of left ventricular reverse remodeling after restrictive annuloplasty. Journal of the American Society of Echocardiography: official publication of the American Society of Echocardiography. 2011;24:1365-1375

7. Tigen K, Karaahmet T, Dundar C, Guler A, Cevik C, Basaran O, Kirma C, Basaran Y. The importance of papillary muscle dyssynchrony in predicting the severity of functional mitral regurgitation in patients with non-ischaemic dilated cardiomyopathy: A two-dimensional speckle-tracking echocardiography study. European journal of echocardiography: the journal of the Working Group on Echocardiography of the European Society of Cardiology. 2010;11:671-676

8. de Isla LP, de Agustin A, Rodrigo JL, Almeria C, del Carmen Manzano M, Rodriguez E, Garcia A, Macaya C, Zamorano J. Chronic mitral regurgitation: A pilot study to assess preoperative left ventricular contractile function using speckle-tracking echocardiography. Journal of the American Society of Echocardiography: official publication of the American Society of Echocardiography. 2009;22:831-838

9. Gelsomino S, Lorusso R, Caciolli S, Capecchi I, Rostagno C, Chioccioli M, De Cicco G, Bille G, Stefano P, Gensini GF. Insights on left ventricular and valvular mechanisms of recurrent ischemic mitral regurgitation after restrictive annuloplasty and coronary artery bypass grafting. The Journal of thoracic and cardiovascular surgery. 2008;136:507-518

10. Calafiore AM, Di Mauro M, Gallina S, Di Giammarco G, laco AL, Teodori G, Tavarozzi I. Mitral valve surgery for chronic ischemic mitral regurgitation. The Annals of thoracic surgery. 2004;77:1989-1997

11. Zoghbi WA, Enriquez-Sarano M, Foster E, Grayburn PA, Kraft CD, Levine RA, Nihoyannopoulos $P$, Otto CM, Quinones MA, Rakowski H, Stewart WJ, Waggoner A, Weissman NJ. Recommendations for evaluation of the severity of native valvular regurgitation with two-dimensional and doppler echocardiography. Journal of the American Society of Echocardiography: official publication of the American Society of Echocardiography. 2003;16:777-802

12. Schiller NB, Shah PM, Crawford M, DeMaria A, Devereux R, Feigenbaum H, Gutgesell H, Reichek N, Sahn D, Schnittger I, et al. Recommendations for quantitation of the left ventricle by two-dimensional echocardiography. American society of echocardiography committee on standards, subcommittee on quantitation of two-dimensional echocardiograms. Journal of the American Society of Echocardiography: official publication of the American Society of Echocardiography. 1989;2:358-367 
13. Kono T, Sabbah HN, Rosman H, Alam M, Jafri S, Goldstein S. Left ventricular shape is the primary determinant of functional mitral regurgitation in heart failure. Journal of the American College of Cardiology. 1992;20:1594-1598

14. Cerqueira MD, Weissman NJ, Dilsizian V, Jacobs AK, Kaul S, Laskey WK, Pennell DJ, Rumberger JA, Ryan T, Verani MS. Standardized myocardial segmentation and nomenclature for tomographic imaging of the heart: A statement for healthcare professionals from the cardiac imaging committee of the council on clinical cardiology of the american heart association. Circulation. 2002;105:539-542

15. Tei C, Ling LH, Hodge DO, Bailey KR, Oh JK, Rodeheffer RJ, Tajik AJ, Seward JB. New index of combined systolic and diastolic myocardial performance: A simple and reproducible measure of cardiac function-a study in normals and dilated cardiomyopathy. Journal of cardiology. 1995;26:357-366

16. Yiu SF, Enriquez-Sarano M, Tribouilloy C, Seward JB, Tajik AJ. Determinants of the degree of functional mitral regurgitation in patients with systolic left ventricular dysfunction: A quantitative clinical study. Circulation. 2000;102:1400-1406

17. Otsuji Y, Handschumacher MD, Schwammenthal E, Jiang L, Song JK, Guerrero JL, Vlahakes GJ, Levine RA. Insights from three-dimensional echocardiography into the mechanism of functional mitral regurgitation: Direct in vivo demonstration of altered leaflet tethering geometry. Circulation. 1997;96:1999-2008

18. Agricola E, Oppizzi M, Maisano F, De Bonis M, Schinkel AF, Torracca L, Margonato A, Melisurgo G, Alfieri O. Echocardiographic classification of chronic ischemic mitral regurgitation caused by restricted motion according to tethering pattern. European journal of echocardiography: the journal of the Working Group on Echocardiography of the European Society of Cardiology. 2004;5:326-334

19. Godley RW, Wann LS, Rogers EW, Feigenbaum H, Weyman AE. Incomplete mitral leaflet closure in patients with papillary muscle dysfunction. Circulation. 1981;63:565-571

20. Van Garsse L, Gelsomino S, Luca F, Lorusso R, Rao CM, Stefano P, Maessen J. Importance of anterior leaflet tethering in predicting recurrence of ischemic mitral regurgitation after restrictive annuloplasty. The Journal of thoracic and cardiovascular surgery. 2012;143:S54-S59

21. Gelsomino S, Lorusso R, De Cicco G, Bille G, Caciolli S, Rostagno C, Capecchi I, Chioccioli M, Stefano P, Gensini GF. Does preoperative tethering symmetry affect left ventricular reverse remodeling after restrictive annuloplasty? International journal of cardiology. 2010;141:182-191

22. Agricola E, Oppizzi M, Galderisi M, Pisani M, Meris A, Pappone C, Margonato A. Role of regional mechanical dyssynchrony as a determinant of functional mitral regurgitation in patients with left ventricular systolic dysfunction. Heart. 2006;92:1390-1395

23. Bleeker GB, Holman ER, Steendijk P, Boersma E, van der Wall EE, Schalij MJ, Bax JJ. Cardiac resynchronization therapy in patients with a narrow qrs complex. Journal of the American College of Cardiology. 2006;48:2243-2250

24. Penicka M, Bartunek J, Lang O, Medilek K, Tousek P, Vanderheyden M, De Bruyne B, Maruskova M, Widimsky $P$. Severe left ventricular dyssynchrony is associated with poor prognosis in patients with moderate systolic heart failure undergoing coronary artery bypass grafting. Journal of the American College of Cardiology. 2007;50:1315-1323

25. Breithardt OA, Sinha AM, Schwammenthal E, Bidaoui N, Markus KU, Franke A, Stellbrink C. Acute effects of cardiac resynchronization therapy on functional mitral regurgitation in advanced systolic heart failure. Journal of the American College of Cardiology. 2003;41:765-770

26. Cleland JG, Daubert JC, Erdmann E, Freemantle N, Gras D, Kappenberger L, Tavazzi L. The effect of cardiac resynchronization on morbidity and mortality in heart failure. The New England journal of medicine. 2005;352:1539-1549

27. Celikyurt U, Vural A, Sahin T, Kilic T, Agacdiken A, Ural D. Relationship between left ventricular dyssynchrony and reverse remodeling after cardiac resynchronization therapy. Clinical cardiology. 2011;34:645-648

28. Gelsomino S, Lorusso R, Capecchi I, Rostagno C, Romagnoli S, Bille G, De Cicco G, Tetta C, Stefano P, Gensini GF. Left ventricular reverse remodeling after undersized mitral ring annuloplasty in patients with ischemic regurgitation. The Annals of thoracic surgery. 2008;85:1319-1330 



\section{Chapter 7}

Left ventricular strain in chronic ischemic mitral regurgitation in relation to mitral tethering pattern

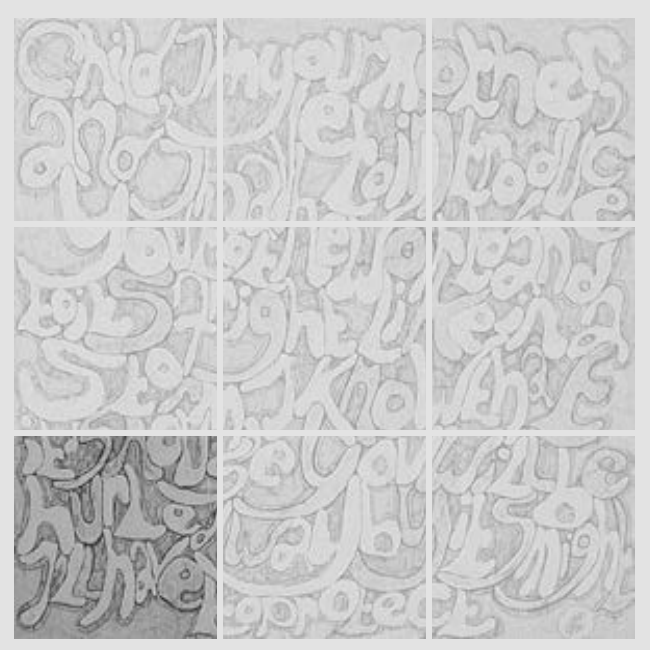

Sandro Gelsomino, MD ,PhD, Leen Van Garsse, MD , Fabiana Lucà, MD, Orlando Parise, MSc, Emile Cheriex, MD, PhD, Carmelo Massimiliano Rao, MD, Gian Franco Gensini, MD, Jos Maessen, MD, PhD.

Published in: J Am Soc Echocardiogr. 2013 Feb 14. In Press. 


\section{Abstract}

Background: In this retrospective study we explored whether different tethering patterns in chronic ischemic mitral regurgitation (CIMR) have a different distribution of LV systolic longitudinal, circumferential and radial strain before and after mitral valve repair.

Methods: Sixty-one CIMR patients who underwent mitral repair were divided on the basis of the preoperative anterior/posterior tethering angle ratio $\alpha / \beta$ (cut-off value 0.76). There were 29 patients with symmetric (Group 1) and 32 with asymmetric preoperative tethering pattern (Group 2). Assessment of longitudinal peak systolic strain was performed off-line by applying speckle tracking imaging to the apical two-threeand four-chamber views of the LV. Peak systolic radial and circumferential strain were obtained from short axis views at basal, middle and apical level. Twenty healthy subjects were controls.

Results: In Group 1 baseline LV strain was impaired in all LV segments with the worst values in the anterolateral, anterior and inferolateral segments at mid-ventricular and basal level. In contrast, asymmetric patients showed higher values in the inferior and inferoseptal walls and values closer to normal in the other segments. After surgery, all strain measurements showed a significant improvement in all LV segments in Group 2 whereas, in Group 1 it worsened in the inferoseptal, inferior and anteroseptal walls and it did not change in the other segments.

Conclusions: Patients with a baseline symmetric tethering pattern showed a more extensive abnormal strain which was observed in all LV segments and was not reverted by surgery. Our findings need to be confirmed by further larger studies. 


\section{Introduction}

Myocardial strain echocardiography has been introduced as a clinical index of region$\mathrm{al}^{1,2}$ and global ${ }^{3,4}$ left ventricular (LV) function. Two-dimensional speckle-tracking echocardiography (2D-STE) measures strain by tracing tissue scatter in grayscale images and enables the angle-independent assessment of myocardial deformation indices ${ }^{5}$. As it is noninvasive and reproducible, strain might be well suited for follow-up and to guide the timing of surgical intervention ${ }^{6-9}$.

In our previous experiences, measures of leaflet tethering resulted in fundamental findings to identify patients with chronic ischemic mitral regurgitation (CIMR) who can really benefit from restrictive annuloplasty. Specifically, preoperative symmetric tethering with anterior mitral leaflet predominance was strongly associated with recurrence of mitral regurgitation and lack of reverse remodeling ${ }^{10-12}$.

In the present study we test the hypothesis that different tethering patterns in CIMR exhibit a different distribution of LV systolic longitudinal, circumferential and radial strain before and after mitral valve repair.

\section{Materials and methods}

\section{Subjects}

Patients with CIMR who survived combined coronary artery bypass grafting (CABG) and undersized mitral ring annuloplasty (UMRA) performed at our Institution (Academic Hospital Maastricht, the Netherlands) between May 2010 and March 2012 were retrospectively included in the study. Inclusion and exclusion criteria are shown in Figure 1. The final study population consisted of 61 patients who were divided based on the preoperative anterior/posterior tethering angle ratio $\alpha / \beta$. A cut-off value of 0.76 was chosen based on our previous experience ${ }^{12}$. There were 29 patients with symmetric (Group 1 , mean $\alpha / \beta=0.83 \pm 0.5$ ) and 32 with asymmetric preoperative tethering pattern (Group 2, mean $\alpha / \beta=0.63 \pm 0.3$ ).

Finally, strain data of CIMR patients were compared with data obtained from 20 healthy controls. The group of healthy controls comprised individuals matched for age, gender, body surface area and body mass index who were volunteers recruited through general practitioners or subjects referred for echocardiography as part of a routine check-up during the study period. Those individuals who showed LV dilatation, had known hypertension or were referred for echocardiographic evaluation of known valvular disease, murmur or heart failure were excluded. Accordingly, all individuals included in the control Group had normal echocardiography. 


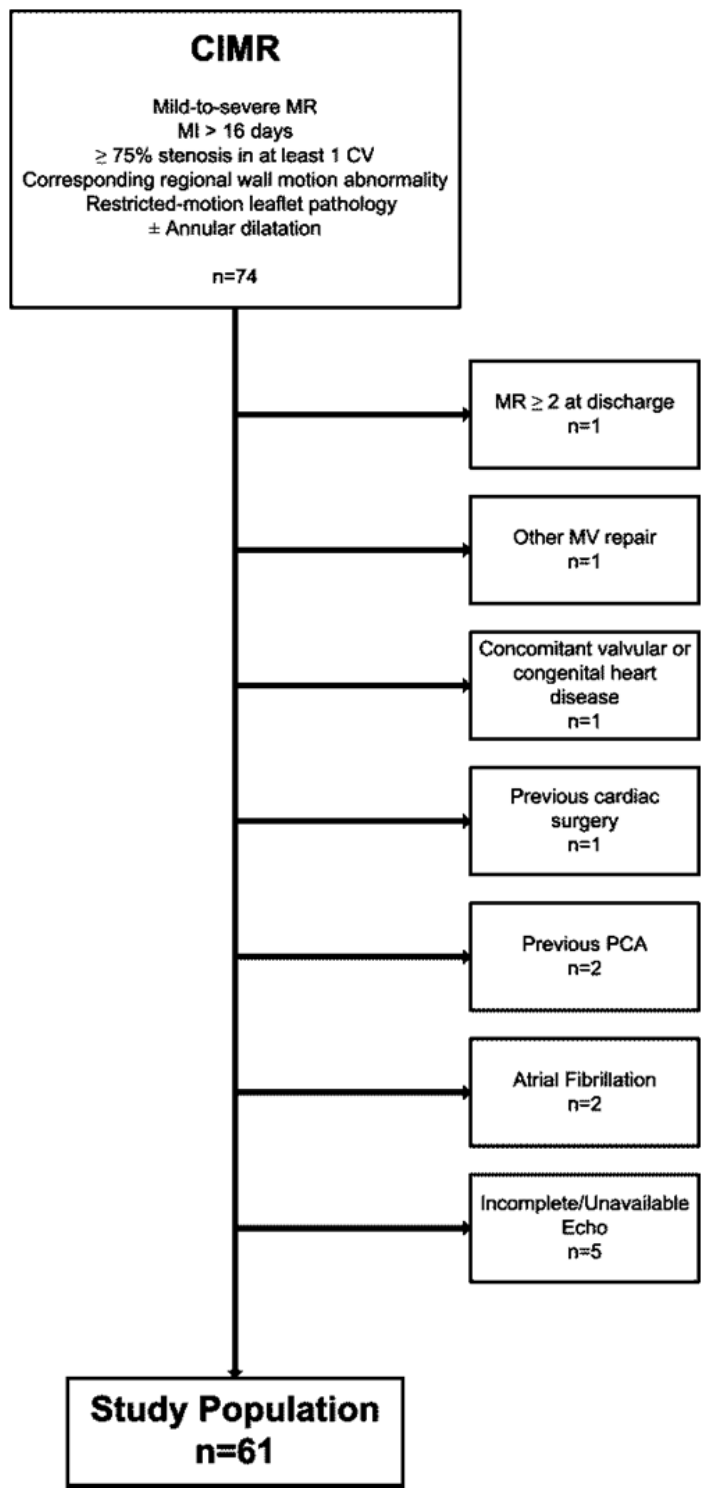

Figure 1. Patients Selection. Patients were included on the basis of the following criteria: (1) mild-to-severe MR with prior myocardial infarction $>16$ days; (2) $75 \%$ or greater stenosis of at least one coronary vessel; (3) a corresponding regional wall motion abnormality; (4) restricted-motion type IIIb (Carpentier's classification) leaflet dysfunction with or without annular dilatation. Exclusion criteria were: (1) Persistent/residual MR ( $M R \geq 2+$ at discharge); (2) other concomitant mitral valve repair procedures performed; (3) degenerative or other non-ischemic etiology or concomitant valvular or congenital heart diseases; (4) previous cardiac surgery/percutaneous transluminal coronary angioplasty; (5) atrial fibrillation; (6) echocardiograms not available/incomplete or images not appropriate for 2D-STE analysis.

Abbreviations. $\mathrm{CIMR}=$ Chronic Ischemic Mitral Regurgitation; $\mathrm{MR}=$ Mitral Regurgitation, $\mathrm{CV}=$ Coronary Vessel; $\mathrm{PCA}=$ Percutaneous Coronary Angioplasty.

\section{Surgery}

All patients underwent complete revascularization. The ring size was determined by standard measurements of the inter-trigonal distance and anterior leaflet height. Downsizing by 2 ring sizes was performed in all patients. A successful repair was assessed as leaflet coaptation of $0.8 \mathrm{~cm}$ or more, MR of 1 or less and a diastolic mitral valve (MV) area exceeding $2 \mathrm{~cm}^{2}$ at intraoperative transesophageal echocardiography. 


\section{Echocardiography}

Transthoracic echocardiography (TTE) was carried out following a standard protocol before surgery (within a week before), at one year follow up and yearly thereafter. Echocardiographic examinations were performed using a commercially available ultrasound system IE 33 (Philips Medical System, Best, The Netherlands). All patients reached one-year follow up. Median follow-up was 16.6 months (Interquartile range [IQR]14.1-20.5).

Images were acquired by experienced echocardiographers, (F.L. and C.M.R.) blinded to the aims of the study, stored in DICOM format and transferred to a workstation for further off-line analysis (TOMTEC Imaging system. Unterschleißheim. Germany).

\section{Standard echocardiography}

Quantitation of MR was carried out as previously described ${ }^{10}$ and the respective thresholds for mild, moderate, and severe MR followed the American Society of Echocardiography (ASE) recommendations ${ }^{13}$. Recurrent MR at latest follow-up was defined as insufficiency $\geq 2+$ in patients with no or trivial MR at discharge.

The anterior mitral leaflet $(\mathrm{AML})$ tethering angle $\alpha$ (between the annular plane and the basal part of the anterior mitral leaflet) and the posterior mitral leaflet (PML) tethering angle $\beta$ (between the annular plane and the basal part of the posterior mitral leaflet) were directly measured with specific software (Philips DICOM Viewer, Philips Medical System, Amsterdam, The Netherlands) ${ }^{10}$.

The ratio of $\alpha$ to $\beta$ was a quantitative measurement of tethering: the closer the ratio was to 1 , the more symmetric was the tethering.

Tenting area (TA) was measured by the area enclosed between the annular plane and mitral leaflets from the parasternal long-axis view at mid-systole. Coaptation height $(\mathrm{CH})$ was measured as the perpendicular distance between the coaptation point of the mitral leaflets and the line connecting the annular hinge points in the long axis view at end-systole.

LV volumes and LV ejection fraction (LVEF) were assessed by the apical biplane method of discs ${ }^{14}$. The wall motion score index (WMSI) was calculated according to a 16-segment model ${ }^{15}$. In addition, the WMSI of the basal and mid-posterior and inferior segments for the posteromedial papillary muscle (PMPM-WMSI) and basal and midlateral and anterior segments for the anterolateral papillary muscle (ALPM-WMSI) were also calculated ${ }^{16}$.

\section{Two-dimensional speckle-tracking echocardiography}

The assessment of longitudinal peak systolic strain was performed off-line by applying 2D-STE to the apical two- three- and four-chamber views of the LV employing a com- 
mercially available software (Tomtec, Tomtec Imaging system, Unterschleißheim, Germany).

With the beginning of the QRS complex and the aortic valve closure time as reference points, the peak longitudinal strain was the maximal negative strain value during the ejection phase. Basal, mid-ventricular and apical longitudinal strains were obtained from each LV wall (infero-septum, anterolateral, inferior, anterior, inferolateral and antero-septum) $)^{17}$.

In addition, peak systolic radial and circumferential strains were obtained from short axis views at basal, middle and apical level ${ }^{18}$.

\section{Statistical analysis}

Data are presented as mean and standard deviations or as median and interquartile range (IQR) after being controlled for normal distribution by the Kolmogorov-Smirnov test. Data were compared for statistical significance using a t test, Mann-Whitney rank sum test and $\chi 2$ test or Fisher exact test where appropriate. Multiple comparisons were carried out by ANOVA with Tukey's post-hoc test.

Two-way ANOVA for repeated measures was employed for comparison between baseline and postoperative echocardiographic data. Intra- and inter-observer reproducibility of strain measurements by 2D-STE was determined by interclass correlation coefficient and Bland-Altman analysis. Intra-observer reproducibility was determined by repeating strain measurements by one experienced reader in 10 randomly selected patients. A second, blinded experienced reader performed the strain analysis in the same 10 patients, providing the inter-observer reproducibility data.

Statistical analysis was performed using a statistical software program (SPSS. version15.0. SPSS Inc. Chicago. IL. USA).

Ethical Committee approval was waived due to the retrospective analysis of the study according to national laws regulating observational retrospective studies (Dutch WMO law). However, all patients gave their informed consent to access their data for scientific purposes.

\section{Results}

\section{Patient characteristics and clinical results}

Patient characteristics are shown in Table 1. No difference was found between Groups in baseline demographics. Four (13.7\%) patients in the symmetric and two $(6.25 \%)$ in the asymmetric Group experienced postoperative complications $(p=0.33)$. These were low output syndrome $(n=1)$, bleeding $(n=2)$, respiratory failure $(n=1)$, infection $(n=1)$ and pericardial effusion $(n=1)$. The mean length of stay in the intensive care unit $(20$ 
[11 to 30] hours in symmetric vs. 23 [15 to 33] hours in asymmetric, p=0.29) and the inhospital stay (7 [5 to 8] days in symmetric vs. 6 [4 to 7] days in asymmetric, $p=0.82$ ), were comparable in the two Groups. At follow up, recurrence of mitral valve regurgitation (MR $\geq 2+)$ was 68.9\% ( $n=20)$ in Group 1 and $18.7 \%(n=6)$ in Group $2(p<0.001)$. Among patients showing recurrent MR, 20 patients (15 in the symmetric and 5 in the asymmetric Group) were medically treated, whereas 6 ( 5 in the symmetric and 1 in the asymmetric Group) underwent reoperation (mitral valve replacement). Neither endocarditis nor other adverse cardiovascular events occurred during the follow up in the two Groups.

\section{Standard echocardiographic results}

In Group 1, at baseline, end-diastolic diameter $(p=0.003)$, end-systolic diameter ( $p$ $=0.003)$, ESVI $(p<0.001)$, EDVI $(p=0.01)$, WMSI $(p=0.02)$ and ALPM-WMSI $(p=0.01)$ were higher, whereas PMPM-WMSI $(p=0.01)$ and LVEF $(P=0.04)$ were lower, compared to group 2 . In addition, in this group tenting area $(p=0.02)$ and coaptation height $(p=0.02)$ were preoperatively higher. Furthermore, patients with symmetric tethering had higher tethering angle $\alpha(p=0.003)$ but lower tethering angle $\beta(p=0.02)$.

At 1-year follow-up the tethering angle $\alpha$ diminished significantly from baseline in both groups $(p<0.001)$ remaining still higher in the symmetric group $(p<0.001$ versus asymmetric), whereas the tethering angle $\beta$ increased in both groups $(p<0.001$ versus baseline) and was comparable at one-year follow-up ( $p=0.9$ between groups).

Moreover, at the latest evaluation, the asymmetric group had smaller LV systolic and diastolic dimensions $(p<0.001)$ and volumes $(p<0.001)$ and lower global WMSI $(p=$ 0.007). Finally, in both groups, PMPM-WMSI increased significantly at one year ( $p$ $<0.001$ and $p=0.01$ versus baseline in symmetric and asymmetric, respectively), whereas ALPM-WMSI remained constant ( $p>0.9$ and $p=0.06$ versus baseline, in symmetric and asymmetric, respectively). All echocardiographic data are shown in Table 2. 
Table 1. Clinical and echocardiographic characteristics.

\begin{tabular}{|c|c|c|c|c|}
\hline & $\begin{array}{l}\text { Controls } \\
(n=20)\end{array}$ & $\begin{array}{c}\text { Symmetric } \\
(n=29)\end{array}$ & $\begin{array}{l}\text { Asymmetric } \\
\quad(n=32)\end{array}$ & $\mathrm{p}$ \\
\hline \multicolumn{5}{|l|}{ Baseline } \\
\hline Age. y & $66.0 \pm 4.4$ & $66.3 \pm 5.9$ & $67.2 \pm 7.2$ & 0.81 \\
\hline Gender M/F & $14 / 6(70 / 30)$ & $19 / 10(65.5 / 34.5)$ & $21 / 11(65.6 / 34.4)$ & $>0.9$ \\
\hline BSA & $1.85 \pm 1.6$ & $1.86 \pm 1.8$ & $1.88 \pm 1.7$ & 0.9 \\
\hline BMI & $23.3 \pm 1.2$ & $24.7 \pm 1.3$ & $25.6 \pm 1.7$ & 0.87 \\
\hline NYHA class & $1[1-2]$ & $3[3-4]$ & $3[3-4]$ & $>0.9$ \\
\hline CCS angina class & $1[1-2]$ & $2[1-3]$ & $2[1-3]$ & $>0.9$ \\
\hline Euroscore & - & $15.5[11-18]$ & 15.7 [10-19] & 0.84 \\
\hline Hypertension & - & $13(44.8)$ & $16(50.0)$ & 0.69 \\
\hline Diabetes & - & $6(20.6)$ & $10(31.2)$ & 0.36 \\
\hline COPD & - & $3(10.3)$ & $7(21.8)$ & 0.25 \\
\hline Chronic renal disease & - & $4(13.7)$ & $9(28.1)$ & 0.19 \\
\hline Cerebral vascular disease & - & $1(3.4)$ & $4(1.2)$ & 0.24 \\
\hline Peripheral vascular disease & - & $3(10.3)$ & $4(1.2)$ & 0.81 \\
\hline Preoperative IABP & - & $2(6.8)$ & $3(9.3)$ & 0.75 \\
\hline Myocardial infarction & - & & & \\
\hline Inferior/Posterior & - & $12(41.4)$ & $15(46.9)$ & 0.67 \\
\hline Anterior/Septal & - & $2(6.9)$ & $3(9.4)$ & 0.75 \\
\hline Lateral & - & $5(17.2)$ & $2(6.2)$ & 0.21 \\
\hline Combined & - & $10(34.5)$ & $12(37.5)$ & 0.61 \\
\hline Coronary vessel disease & - & $2[1-3]$ & $2[1-3]$ & $>0.9$ \\
\hline Left Main & - & $6(20.6)$ & $7(21.8)$ & $>0.9$ \\
\hline Surgery & - & & & \\
\hline CPB time ( $\min )$ & - & 111 [95-130] & 107 [98-119] & 0.10 \\
\hline $\mathrm{CCL}$ time (min) & - & 99 [79-115] & 93 [80-107] & 0.23 \\
\hline Mitral Ring size (mm) & - & $28[26-30]$ & $28[26-30]$ & 0.9 \\
\hline CABG & - & & & \\
\hline Anastomoses/patient & - & $2[1-3]$ & $2[1-3]$ & $>0.9$ \\
\hline Arterial graft/patient & - & $1[1-2]$ & $1[1-2]$ & $>0.9$ \\
\hline \multicolumn{5}{|l|}{ Follow-up } \\
\hline NYHA class & - & $2[2-3]$ & $1[1-2]$ & $<0.001$ \\
\hline NYHA III/IV & - & $21(72.4)$ & $8(25.0)$ & $<0.001$ \\
\hline
\end{tabular}

Data are shown as mean \pm standard deviation, median [Interquartile range] or number (percentage) as appropriated.

Abbreviations: $\mathrm{M} / \mathrm{F}=$ Male/Female; $\mathrm{BSA}=$ Body Surface Area $\left(\mathrm{m}^{2}\right) ; \mathrm{BMI}=$ Body Mass Index $\left(\mathrm{Kg} / \mathrm{m}^{2}\right) ; \mathrm{NYHA}=$ New York Heart Association; CCS = Canadian Cardiovascular Society; COPD = Chronic obstructive pulmonary disease; $\mathrm{IABP}=$ Intra-aortic balloon pump; $\mathrm{CPB}=$ Cardiopulmonary bypass; $\mathrm{CCL}=$ (Aortic) Cross-clamp; $\mathrm{CABG}$ = Coronary artery bypass grafting. 
Table 2. Standard Echocardiographic Data

\begin{tabular}{|c|c|c|c|c|c|c|c|}
\hline & \multirow[b]{2}{*}{ Controls } & \multicolumn{3}{|c|}{ Symmetric } & \multicolumn{3}{|c|}{ Asymmetric } \\
\hline & & Baseline & Follow-up & $\mathrm{p}$ & Baseline & Follow-up & $p$ \\
\hline MR (grade) & - & $3[3-4]$ & $2[2-4]^{+}$ & 0.02 & $3[3-4]$ & $1[1-2]$ & $<0.001$ \\
\hline $\operatorname{EROA}\left(\mathrm{mm}^{2}\right)$ & - & $38 \pm 12$ & $34 \pm 11$ & 0.13 & $35 \pm 10$ & - & - \\
\hline $\mathrm{RF}(\%)$ & - & $48 \pm 12$ & $44 \pm 13$ & 0.41 & $45 \pm 12$ & - & - \\
\hline $\mathrm{RV}$ (mL/beat) & - & $58 \pm 15$ & $55 \pm 15$ & 0.67 & $56 \pm 13$ & - & - \\
\hline $\mathrm{TA}\left(\mathrm{cm}^{2}\right)$ & $0.8 \pm 0.1$ & $4.1 \pm 1.1^{*+}$ & $3.7 \pm 1.2^{*+}$ & 0.06 & $3.0 \pm 1.0^{*}$ & $1.7 \pm 0.5^{*}$ & $<0.001$ \\
\hline $\mathrm{CH}(\mathrm{mm})$ & $6.2 \pm 2$ & $13.4 \pm 4.2^{*+}$ & $12.8 \pm 4.0^{*+}$ & 0.74 & $9.7 \pm 3.4^{*}$ & $6.9 \pm 2.6^{*}$ & $<0.001$ \\
\hline$\alpha\left({ }^{\circ}\right)$ & $25 \pm 4$ & $45 \pm 9^{*+}$ & $36 \pm 9^{*+}$ & $<0.001$ & $35 \pm 5^{*}$ & $25 \pm 5$ & $<0.001$ \\
\hline$\beta\left({ }^{\circ}\right)$ & $36 \pm 5$ & $41 \pm 12^{*+}$ & $77 \pm 12^{*}$ & $<0.001$ & $55 \pm 9^{*}$ & $77 \pm 11^{*}$ & $<0.001$ \\
\hline$\alpha / \beta$ & $0.6 \pm 0.2$ & $0.8 \pm 0.5^{* \dagger}$ & $0.5 \pm 0.3^{* \dagger}$ & $<0.001$ & $0.6 \pm 0.3^{*}$ & $0.3 \pm 0.1^{*}$ & $<0.001$ \\
\hline $\operatorname{EDD}(\mathrm{mm})$ & $45 \pm 5$ & $69 \pm 8^{*+}$ & $69 \pm 8^{*+}$ & $>0.9$ & $57 \pm 5^{*}$ & $49 \pm 3^{*}$ & 0.007 \\
\hline $\mathrm{ESD}(\mathrm{mm})$ & $29 \pm 4$ & $58 \pm 5^{*+}$ & $57 \pm 7^{*+}$ & 0.9 & $46 \pm 6^{*}$ & $39 \pm 3^{*}$ & 0.005 \\
\hline EDVI $\left(\mathrm{mL} / \mathrm{m}^{2}\right)$ & $56 \pm 8$ & $115 \pm 14^{*+}$ & $113 \pm 12^{*+}$ & 0.85 & $101 \pm 9^{*}$ & $88 \pm 6^{*}$ & $<0.001$ \\
\hline ESVI $\left(\mathrm{mL} / \mathrm{m}^{2}\right)$ & $25 \pm 6$ & $69 \pm 7^{*+}$ & $66 \pm 7^{*+}$ & 0.77 & $59 \pm 5^{*}$ & $47 \pm 4^{*}$ & 0.01 \\
\hline LVEF (\%) & $59 \pm 6$ & $39 \pm 5^{*+}$ & $41 \pm 5$ & 0.34 & $44 \pm 4^{*}$ & $46 \pm 4^{*}$ & 0.8 \\
\hline WMSI & $1.0 \pm 0$ & $1.4 \pm 0.5^{*+}$ & $1.6 \pm 0.4^{*+}$ & 0.01 & $1.2 \pm 0.4^{*}$ & $1.0 \pm 0.3$ & 0.01 \\
\hline PMPM-WMSI & $1.0 \pm 0$ & $2.1 \pm 0.3^{*+}$ & $2.6 \pm 0.3^{*}$ & $<0.001$ & $2.5 \pm 0.4^{*}$ & $2.7 \pm 0.3^{*}$ & 0.01 \\
\hline ALPM-WMSI & $1.0 \pm 0$ & $2.5 \pm 0.4^{*+}$ & $2.5 \pm 0.4^{*+}$ & $>0.9$ & $1.2 \pm 0.2^{*}$ & $1.1 \pm 0.2^{*}$ & 0.06 \\
\hline
\end{tabular}

Data are shown as mean \pm standard deviation median [Interquartile range] or number (percentage) as appropriated.

Abbreviations. $\mathrm{MR}=$ Mitral Regurgitation; $\mathrm{EROA}=$ Effective regurgitant Orifice Area; $\mathrm{RF}=$ Regurgitant Fraction; $\mathrm{CH}=$ Coaptation height; $\alpha=$ anterior mitral leaflet tethering angle; $\beta=$ posterior mitral leaflet tethering angle; $\mathrm{EDD}=$ End Diastolic Diameter; ESD = End Systolic Diameter; EDVI = End Diastolic Volume Index; ESVI = End Systolic Volume Index; LVEF = Left Ventricular Ejection Fraction; WMSI = Wall motion score Index; PMPM = Posteromedial papillary muscle; ALPM = Anterolateral papillary muscle. ${ }^{*} \mathrm{p}<0.05$ vs. controls, ${ }^{\dagger}$ $\mathrm{p}<0.05$ vs. asymmetric.

\section{Longitudinal systolic strain}

At baseline (Figure 2), patients in Group 1 showed abnormal reductions in longitudinal strain values in all LV segments (all, $p<0.001$ ) with the worst values in the midventricular and basal segments of the antero-lateral $(2.3 \pm 2.7 \%$ and $3.3 \pm 2.1 \%$. respectively) anterior ( $3.4 \pm 2.5 \%$ and $3.9 \pm 2.7$, respectively) and inferolateral $(3.3 \pm 2.9$ and $3.5 \pm 2.0 \%$, respectively) walls.

In contrast, patients with asymmetric tethering showed better values in the inferoseptum (apical, $p=0.02$; mid-ventricular and basal, both $p<0.001$ vs. controls) and inferior (all, $p<0.001$ vs. controls) walls. In the other segments peak strain values were comparable to controls in this Group.

At 1-year follow up (Figure 3), in patients with asymmetric tethering, longitudinal systolic strain showed a significant improvement in all LV segments (all, $p<0.05$ ). In contrast, In Group 1, these values worsened in the mid-ventricular and basal segments 
of the inferoseptal ( $p=0.02$ and $p=0.01$, respectively), inferior $(p=0.02$ and $p=0.001$, respectively) and anteroseptal (both, $p<0.001$ ) walls remaining unchanged in the other segments (Figure 4).
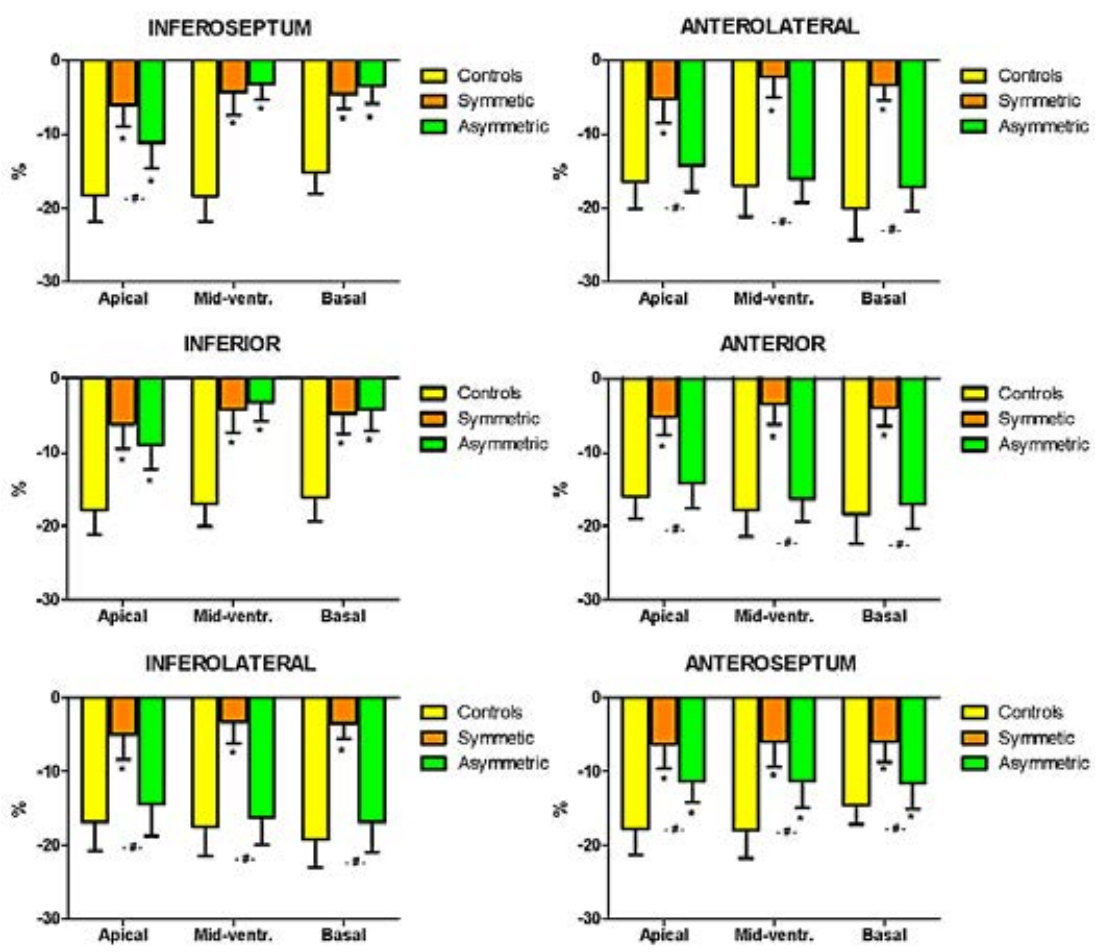

Figure 2. Baseline longitudinal segmental strain in the two Groups .Patients with symmetric tethering showed higher (less negative) values in all segments, whereas those with asymmetric tethering had highest values in the inferoseptal and inferior walls. ${ }^{*}$ Significance vs. controls; ${ }^{\#}$ significance symmetric vs. asymmetric. 

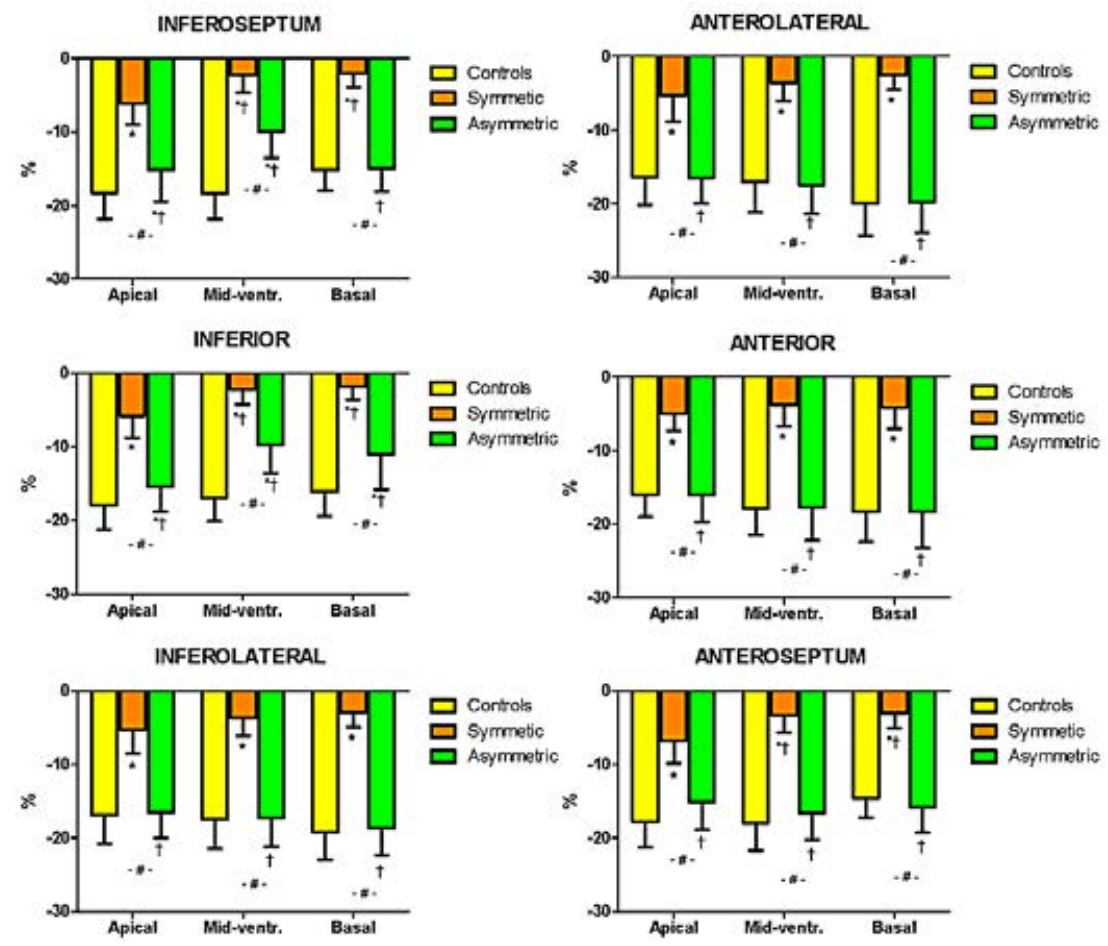

Figure 3. Longitudinal segmental strain at the follow up in the two Groups. In asymmetric patients it improved significantly in all segments. In contrast, in symmetric patients it worsened at level of inferoseptal, inferior and anteroseptal segments in the mid-ventricular and basal walls. "Significance vs. controls; ${ }^{\#}$ significance symmetric vs. asymmetric; † significance vs. baseline values.

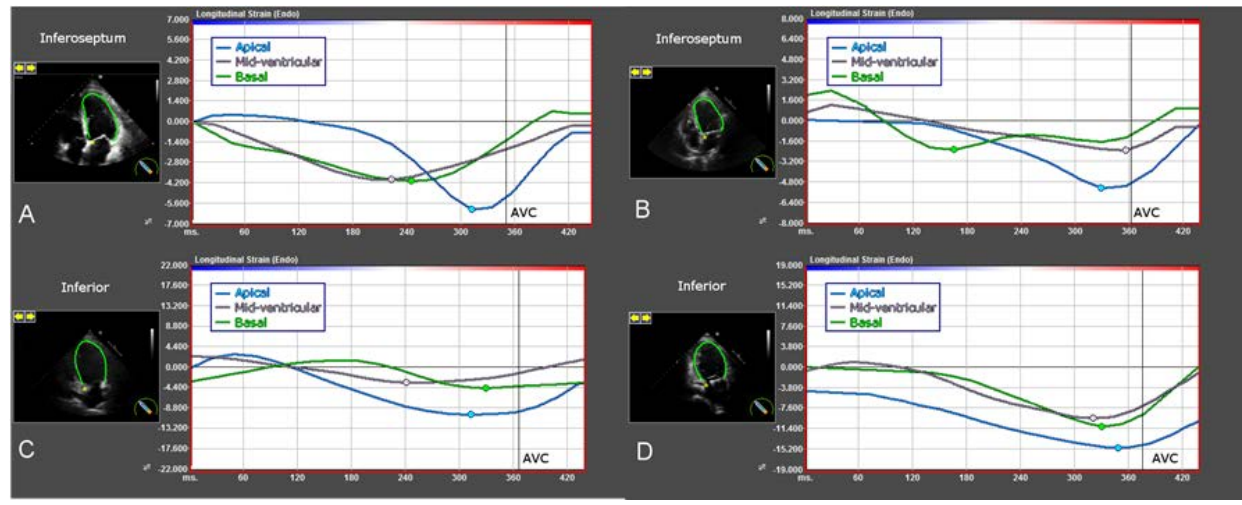

Figure 4. A) Baseline inferoseptal longitudinal strain in a patient with symmetric tethering. B) Inferoseptal longitudinal strain in the same patient as in A at follow up: mid-ventricular and basal segments showed a significant worsening. C) Baseline inferior longitudinal strain in a patient with asymmetric tethering. D) Inferior longitudinal strain in the same patient as in C, at follow up: it significantly improved in all segments. Abbreviations. AVC: Aortic Valve Closure. 


\section{Circumferential systolic strain}

Baseline circumferential strain (Figure 5) was significantly worse in symmetric patients in the anterolateral and anterior segments in all short axis views (all. $p<0.001$ ) and also in inferolateral and anteroseptal segments at basal and mid-ventricular level $(p<0.001)$. In contrast, patients with asymmetric tethering showed better circumferential strain values in the inferoseptal and inferior walls at basal and mid ventricular level $(p<0.001$ vs. controls).

At one-year follow-up control (Figure 6) in patients in Group 2, circumferential strain improved in all segments (all, $\mathrm{p}<0.05$ ) whereas in symmetric patients it did not change in the anterolateral, anterior and inferolateral walls while it significantly worsened $(p<0.05)$ in the inferoseptum (all views), inferior wall (all views) and anteroseptum (basal and mid-ventricular views).

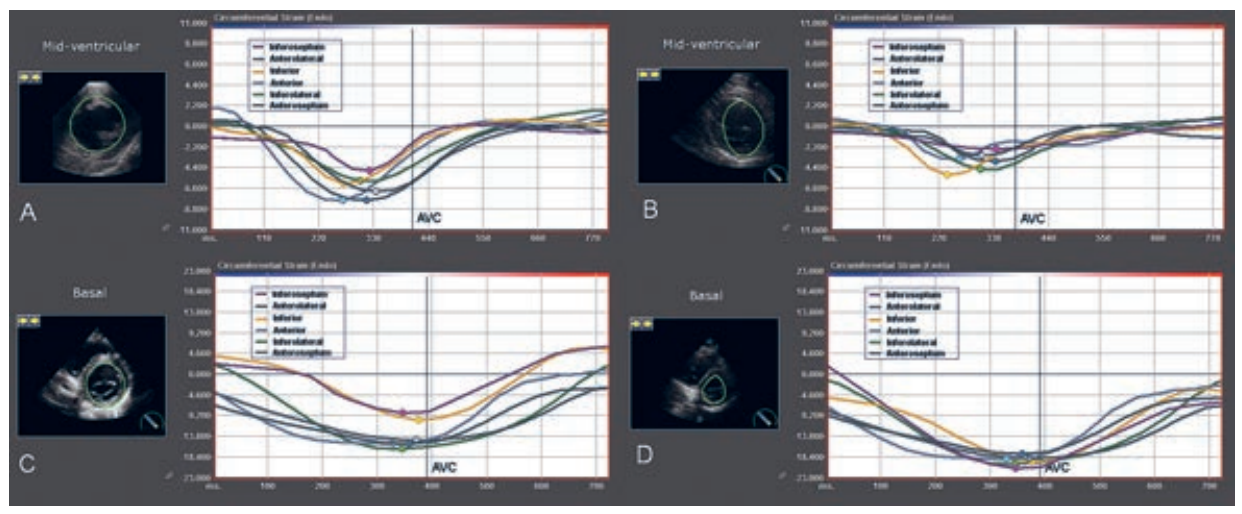

Figure 6 A) Baseline mid-ventricular short axis circumferential strain in a patient with symmetric tethering. B) Mid-ventricular short axis circumferential strain in the same patient as in A, at follow up: all segments showed a significant worsening. C) Baseline basal short-axis circumferential strain in a patient with asymmetric tethering. D) Follow-up short-axis circumferential strain in the same patient as in C: strain significantly improved in all segments.

Abbreviations. AVC: Aortic Valve Closure. 
Basal Baseline Segmental Circumferential Systolic Strain

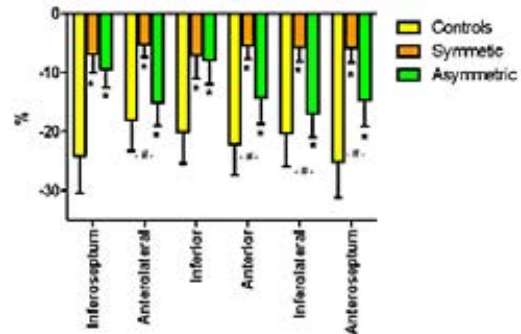

Mid Baseline Segmental Circumferential Systolic Strain

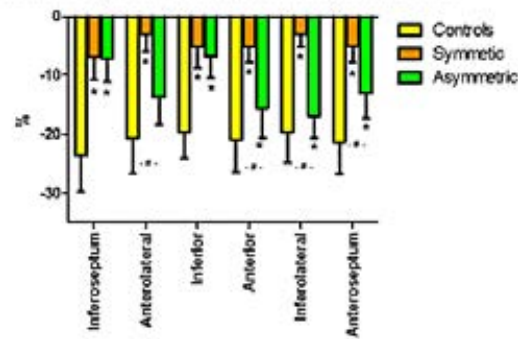

Apical Baseline Segmental Circumferential Systolic Strain

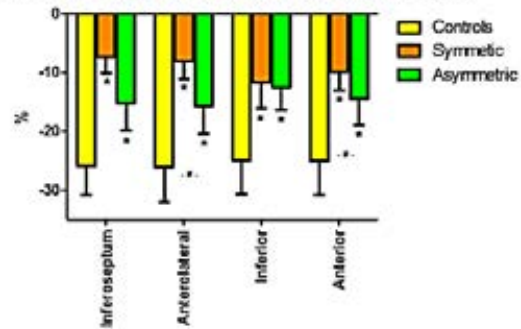

Basal Postoperative Segmental Circumferential Systolic Strain
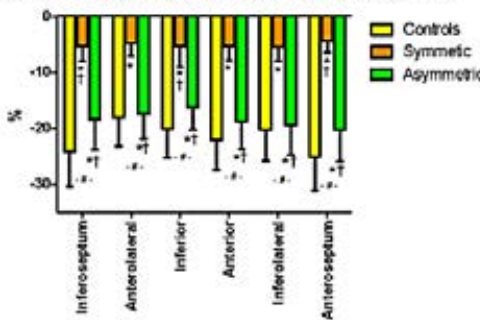

$\square$ Agrmmer

Mid Postoperative Segmental Circumferential Systolic Strain

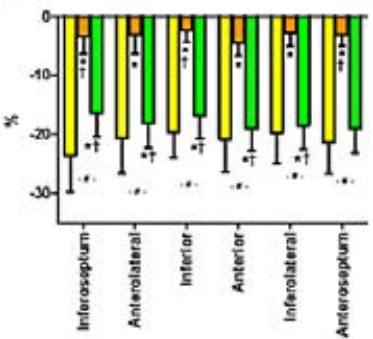

Apical Postoperative Segmental Circumferential Systolic Strain

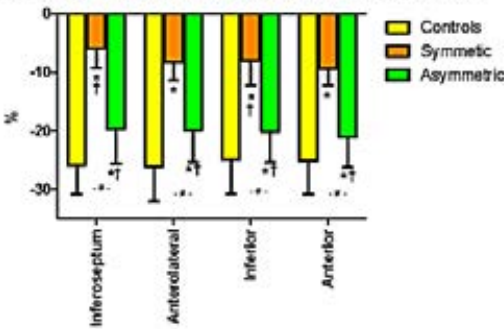

Figure 5. Circumferential strain at baseline and at follows up in the two Groups. Baseline circumferential strain was significantly higher in symmetric patients in the anterolateral and anterior segments in all short axis views and also in inferolateral and anteroseptal segments at basal and mid-ventricular level. At one-year follow-up control in patients in Group 2 circumferential strain improved in all segments whereas in symmetric patients it significantly worsened in the infero-septum, inferior wall and antero-septum. ${ }^{*}$ Significance vs. controls; " significance symmetric vs. asymmetric; † significance vs. baseline values.

\section{Radial systolic strain}

Baseline radial strain (Figure 7) was worse (lower) in symmetric patients in the basal and mid ventricular views at the level of anterolateral, anterior, inferolateral and anteroseptal segments (all, $p<0.001$ ).

In the apical view, this Group showed values lower than controls in all segments (all, $\mathrm{p}<0.001$ ). On the contrary, asymmetric patients exhibited values lower than controls and comparable to symmetric patients in the inferior and inferoseptal segments 
in the basal (inferior, $18.8 \pm 7.7$; inferoseptal, $16.0 \pm 8.4$ ) and mid-ventricular (inferior, $13.4 \pm 9.4$; inferoseptal, $15.6 \pm 8.8$ ) views. Others values and apical strain values were significantly higher (apical inferior, $p=0.007$, others $p<0.001$ ).

At one year, radial systolic strain worsened, in the symmetric Group, in the inferior, inferoseptal and anteroseptal segments in the basal and mid-ventricular views whereas it was unchanged in the other segments. In contrast, radial strain improved in all segments in the asymmetric patients at follow-up control (Figure 8).

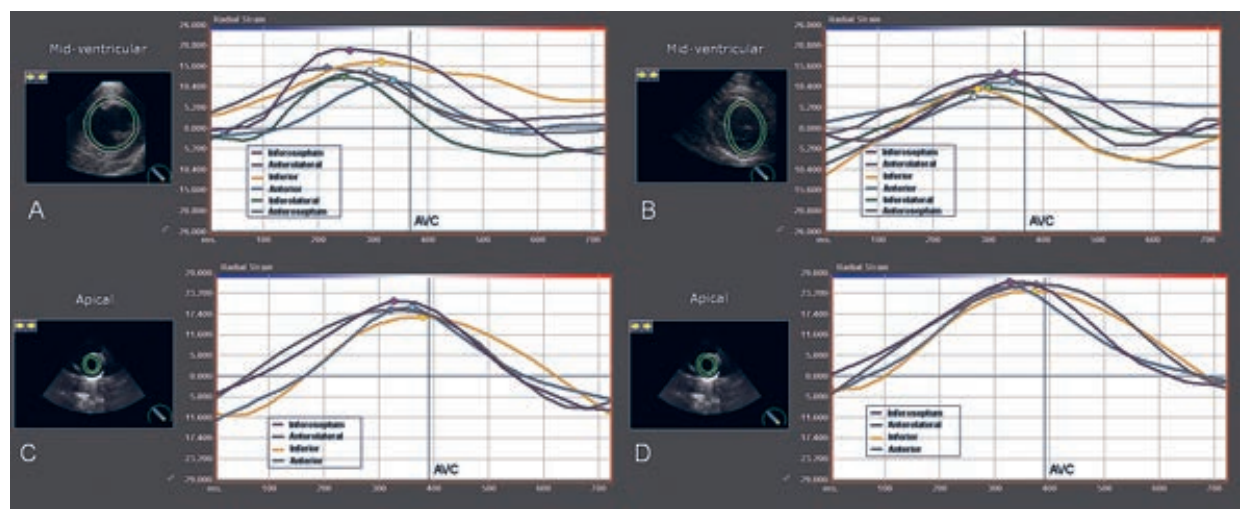

Figure 8. A) Baseline mid-ventricular short axis radial strain in a patient with symmetric tethering. B) Midventricular short axis radial strain in the same patient as in A, at follow up: inferior, inferoseptal and anteroseptal segments showed a significant worsening. C) Baseline apical short axis radial strain in a patient with asymmetric tethering. D) Follow-up apical short axis radial strain in the same patient as in C: strain significantly improved in all segments.

Abbreviations. AVC: Aortic Valve Closure. 
Basal Preoperative Segmental Radial Systolic Strain

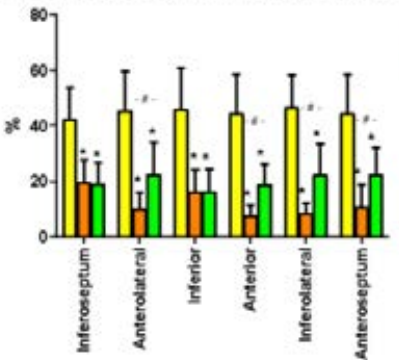

Mid Preoperative Segmental Radial Systolic Strain

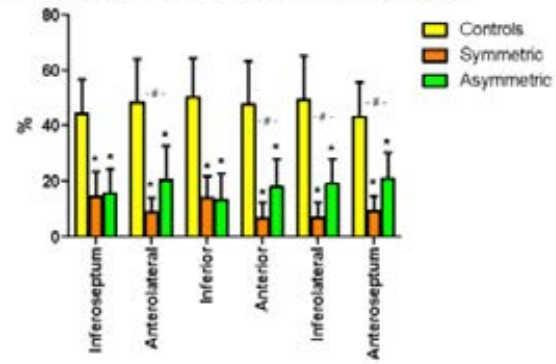

Apical Preoperative Segmental Radial Systolic Strain

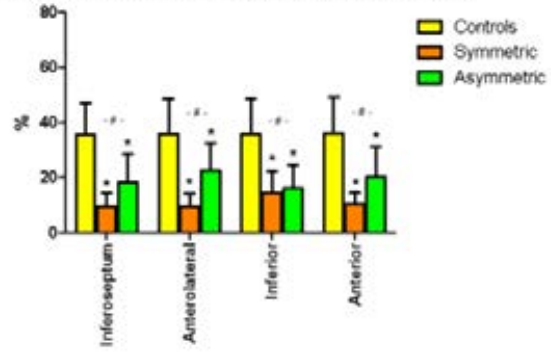

Basal Postoperative Segmental Radial Systolic Strain

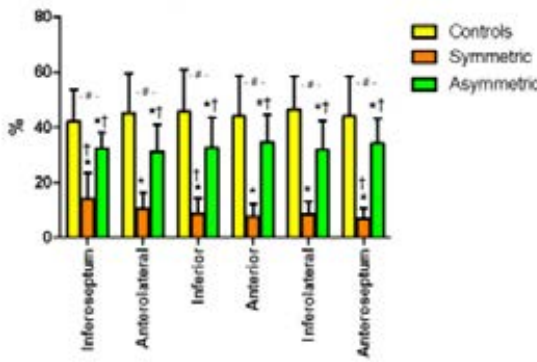

Mid Postoperative Segmental Radial Systolic Strain

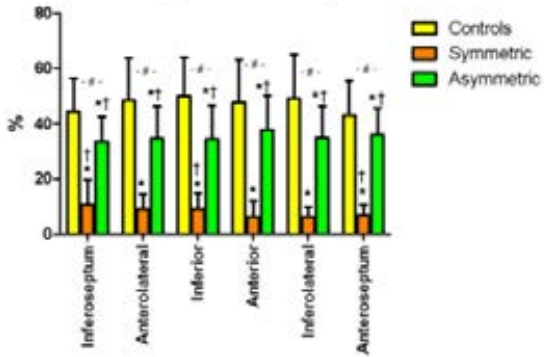

Apical Postoperative Segmental Radial Systolic Strain

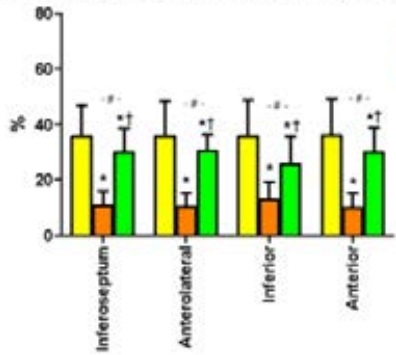

Figure 7. Radial strain at baseline and at follow up in the two Groups. Strain was lower in symmetric patients in the basal and mid ventricular views at the level of the anterolateral, anterior, inferolateral and anteroseptal segments in all short axis views (all, $p<0.001$ ) and in all segments in the apical view. At one year, radial systolic strain worsened, in the symmetric Group, in the inferior, inferoseptal and anteroseptal segments in the basal and mid-ventricular views whereas it was unchanged in the other segments. In contrast, radial strain improved in all segments in the asymmetric patients. "Significance vs. controls; " significance symmetric vs. asymmetric; †significance vs. baseline values. 


\section{Reliability analysis}

Reliability analysis demonstrated good intra- and inter-observer agreement for the measurement of longitudinal, circumferential and radial strain. In addition, BlandAltman analysis showed small bias, with no significant trend for all strain measurements performed by the same observer (Table 3).

Table 3. Intra-observer and inter-observer reproducibility of myocardial strain assessed by two-dimensional speckle-tracking echocardiography

\begin{tabular}{lcccccr}
\hline & \multicolumn{3}{c}{ Intra-observer } & \multicolumn{3}{c}{ Inter-observer } \\
\cline { 2 - 7 } & Mean difference \pm 2SD & ICC & $\mathrm{p}$ & Mean difference \pm 2 SD & ICC & $\mathrm{p}$ \\
\hline Longitudinal strain & $0.04 \pm 0.20$ & 0.96 & $<0.001$ & $0.06 \pm 0.28$ & 0.93 & $<0.001$ \\
Circumferential strain & $-0.19 \pm 2.01$ & 0.98 & $<0.001$ & $0.29 \pm 3.87$ & 0.90 & 0.001 \\
Radial strain & $0.64 \pm 7.44$ & 0.96 & $<0.001$ & $1.80 \pm 10.9$ & 0.80 & 0.006 \\
\hline
\end{tabular}

\section{Discussion}

The main findings of our paper can be summarized as follows: 1) In chronic ischemic mitral regurgitation (CIMR), different preoperative mitral tethering patterns reflect a different extent of myocardial involvement in the ischemic disease and impairment of LV strain. Indeed, symmetric tethering seems to reflect a more generalized disease with significant involvement of all segments of the LV myocardium whereas patients with asymmetric tethering show a more localized worsening of longitudinal, circumferential and radial strain. 2) In asymmetric patients the impairment of LV strain was significantly higher in the inferoseptal and inferior segments whereas symmetric patients showed the worst strain values in the anterolateral, anterior and anterolateral segments. 3) After repair, longitudinal, circumferential and radial strain significantly worsened or remained unchanged in patients with symmetric tethering while in those with asymmetric tethering strain values significantly improved in all segments. 4) Different tethering patterns reflect also a greater extent of global LV remodeling (baseline higher values of WMSI) and local remodeling at level of both papillary muscles (baseline higher PMPM-WMSI and ALPM-WMSI) in the symmetric Group with prevalent remodeling at level of the antero-medial papillary muscle. 5) At follow up in both Groups we observed a significant increase in PMPM-WMSI, demonstrating a substantial continued remodeling at level of the LV surrounding the posteromedial papillary muscle after surgery. 


\section{Myocardial deformation indices and tethering pattern in chronic ischemic mitral regurgitation}

Myocardial deformation indices such as strain and strain rate of the LV obtained using speckle-tracking imaging, have been reported to be useful in detecting subclinical deterioration in LV function ${ }^{19}$ and predicting postoperative LV dysfunction in patients with $\mathrm{MR}^{7,20,21}$. Furthermore, strain was reported to correlate with $\mathrm{dP} / \mathrm{dt}$ and contractile reserve in patients with $\mathrm{MR}^{22,23}$. In addition, results from a study in animals ${ }^{24}$ revealed that transmural strain may be an early marker of LV dysfunction after development of MR and it has been demonstrated that longitudinal speckle tracking-derived strain was lower in patients with asymptomatic MR and normal LV ejection fraction, indicating subclinical dysfunction ${ }^{25}$.

Chronic ischemic mitral regurgitation (CIMR) is a common complication of coronary artery disease that doubles late mortality ${ }^{26,27}$. Despite the clinical importance of $\mathrm{CIMR}$, the therapy remains problematic. Mitral ring annuloplasty, often applied at the time of bypass surgery, reduces mitral annular size but does not directly addresses the broader problem of ischemic LV distortion with tethering; its benefits are therefore incomplete and recurrence of mitral regurgitation after annuloplasty has been widely

reported predominantly related to progressive LV remodeling ${ }^{28-30}$.

The concept of tethering symmetry in ischemic mitral regurgitation was first introduced by Agricola and coworkers ${ }^{31}$ who recognized, among patients with CIMR due to restricted motion, two groups according to the tethering pattern: the asymmetric group with predominant posterior tethering of both leaflets and the symmetric one with predominant apical tethering of both leaflets. They demonstrated that these two groups translate into different degrees of local and global LV remodeling and characteristics of the regurgitant jet. Our previous experiences demonstrated that the preoperative symmetric pattern with anterior mitral leaflet tethering prevalence is strongly associated with lack of reverse remodeling and higher recurrence of MR after annuloplasty $^{11,12}$.

In the present study we calculated anterior and posterior tethering angles with their ratio $\alpha / \beta$ (cut-off $\geq 0.76$ ) being a quantitative measurement of tethering symmetry. We tested whether the distribution of LV systolic deformation indices was different, before and after annuloplasty, in patients with symmetric and asymmetric tethering.

Our findings demonstrate that asymmetric tethering reflects a more localized process at the LV level. Indeed, the peak strain is altered in a restricted area which comprises the insertion of the posterior papillary muscle (mid-ventricular segment(s), inferior-inferoseptal walls). The impairment of peak strain in this area might reflect a local remodeling in this region with altered wall motion ${ }^{12}$ and this is confirmed by the analysis of local remodeling at level of papillary muscle which confirms a prevalent PMPM remodeling. 
Since each papillary muscle supplies chordae to both leaflets, consequentially an infero-posterior displacement of only one papillary muscle invariably exerts traction on both leaflets ${ }^{32}$. Therefore, the different shapes of tethering of the two groups depend on the relationship of the three tethering vectors (infero-posterior, apical and lateral). Hence, we can suppose that in asymmetric tethering, the posterior leaflet is simply drawn more inferiorly (and posteriorly) than apically (more parallel to the posterior wall). This posterior restriction of the posterior leaflet prevents it from reaching its normal, more anteriorly located coaptation point, so that the coaptation point moves posteriorly, creating the asymmetric tethering shape.

In contrast, symmetric tethering seems to reflect generalized disease with significant involvement of all segments of the LV myocardium. As a result, in these patients there is additional apical and medio-lateral tethering in addition to the posterior component. The analysis of strain confirms this hypothesis and the examination of local remodeling shows an involvement of LV surrounding both papillary muscle but with higher values of ALPM-WMSI.

The net result of these forces is a more apical tenting, with the coaptation point being displaced more apically with the regurgitant jet which usually has a central origin and direction being the systolic motion of both leaflets equally affected ${ }^{33}$.

Patients in Group 1 experienced a high recurrence of MR after surgery and our data confirm that it is related to continued remodeling which is detected especially at level of the PMPM ${ }^{30}$. Nonetheless, this is observed also in the asymmetric Group in which the MR recurrence rate was low and this suggests that other mechanisms are involved in the recurrence of MR in these patients now confirmed by a recent study demonstrating that MR recurrence may occur also in the absence of progressive LV remodeling ${ }^{34}$.

However, the question arises if these two patterns are two different expressions of the same disease or if they represent two sequential steps of CIMR. In the latter case symmetric tethering might reflect an advanced stage of the disease resulting from progressive involvement of neighboring myocardium. This aspect deserves further investigations and will be the object of an ongoing study.

Nonetheless, our results confirm that mitral repair is more likely to fail in patients with symmetric tethering. Therefore, in such patients, concomitant or alternative surgery addressing leaflet tethering ${ }^{33}$ or a chordal-sparing mitral valve replacement should be considered ${ }^{35}$.

Tethering symmetry may, therefore, represent an easy and immediate tool to identify patients who cannot benefit from UMRA. Thus, an accurate evaluation of tethering pattern before surgery should be incorporated into risk prediction models for unsuccessful MV repair and, in patients with a preoperative symmetric tethering pattern, the risk of performing UMRA must be carefully weighed. 


\section{Study limitations}

The main limitations of the present study are its retrospective nature and the lack of information on myocardial viability. Therefore, the fact that patients with asymmetric tethering showed improved LV strain post-operatively could be related to a higher rate of preoperative LV viability in this group. This aspect deserves further investigation and will be object of an on-going study. Furthermore, patients with symmetric tethering had larger LV volumes than those with asymmetric tethering and we cannot exclude that symmetric tethering might be simply an indicator that LV geometric abnormalities are more widespread.

Second, postoperative evaluation of the coronary status was not assessed. It would have been helpful to differentiate between surgical failure (valve repair and CABG) and progress of the coronary disease.

Third, the PISA method of assessing the severity of ischemic MR is less accurate as the PISA is less likely to be a hemisphere. Adjustments of the aliasing velocity were carried out such that a well-defined hemisphere was obtained. This was done by shifting the baseline toward the direction of the flow, or by lowering the Nyquist limit or both (the latter reduces the wall filter whereas the former does not) ${ }^{13}$.

Fourth, as the PISA method overestimates MR in patients with eccentric jets ${ }^{36}$, we therefore employed also pulsed Doppler quantitative flow methods and, in case of contradictory results, PISA was chosen in the presence of central jet or calcific mitral valve/mitral annulus, whereas pulsed Doppler quantitative flow methods were preferred when the jet was eccentric or multiple.

Fifth, as myocardial movement is basically three-dimensional thus including rotational movements, two-dimensional speckle tracking may have inherent limitations, especially in patients with dilated LV.

Finally, strain echocardiographic measurements are not widely validated, are dependent on image quality and a major concern remains about their reproducibility.

\section{Conclusions}

Among patients with ischemic mitral regurgitation, those with baseline symmetric tethering pattern showed more extensive abnormal peak strains which were observed in all LV segments. These abnormalities were not reverted by annuloplasty. In contrast, in asymmetric patients peak systolic longitudinal strain parameters were altered only in the inferior and inferoseptal walls and returned to normal values after surgery. There is the need for extensive prospective studies to confirm our findings. 


\section{Appendix 1: Supplemental figures}

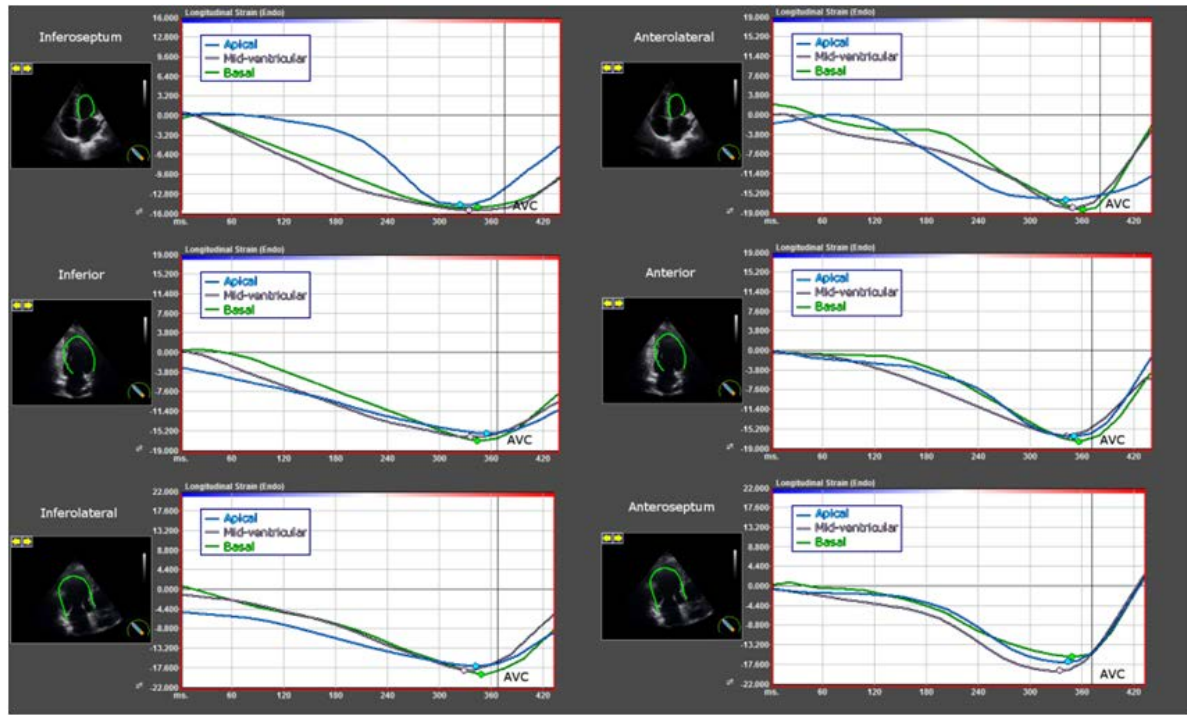

Supplemental Figure 1. Segmental longitudinal strain in a healthy control.

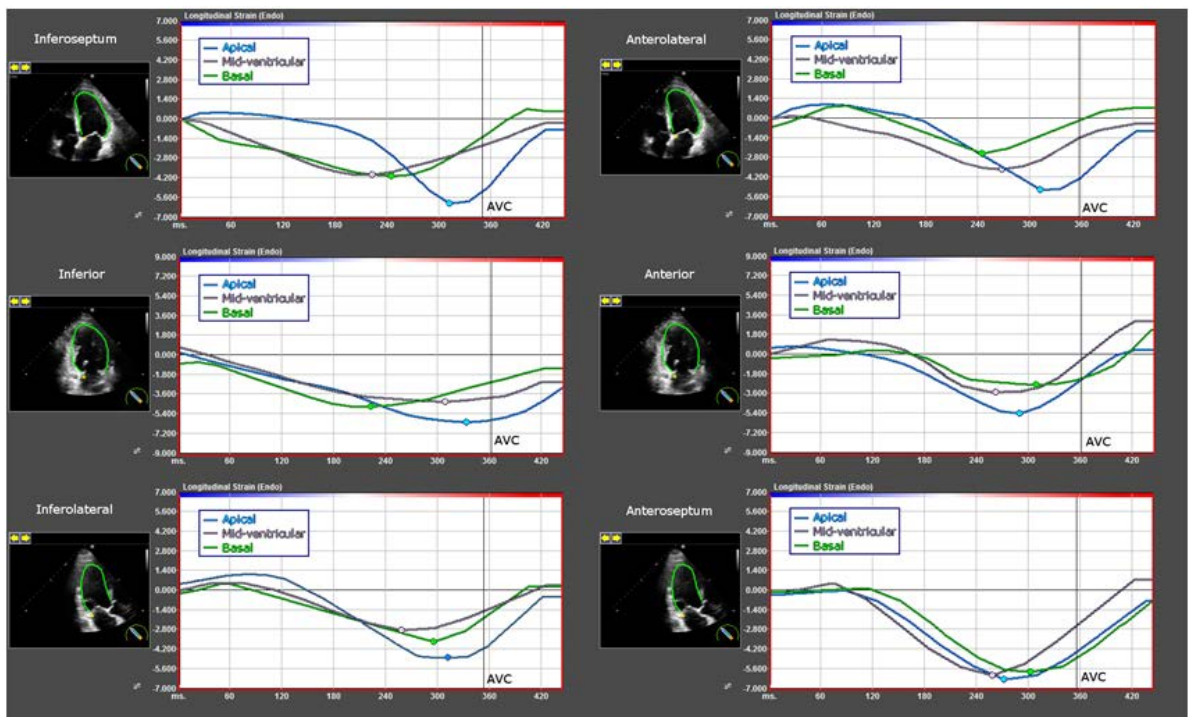

Supplemental Figure 2. Baseline segmental longitudinal strain in a patient with preoperative symmetric tethering. This patients showed higher (less negative) values in all segments. AVC: Aortic Valve Closure. 


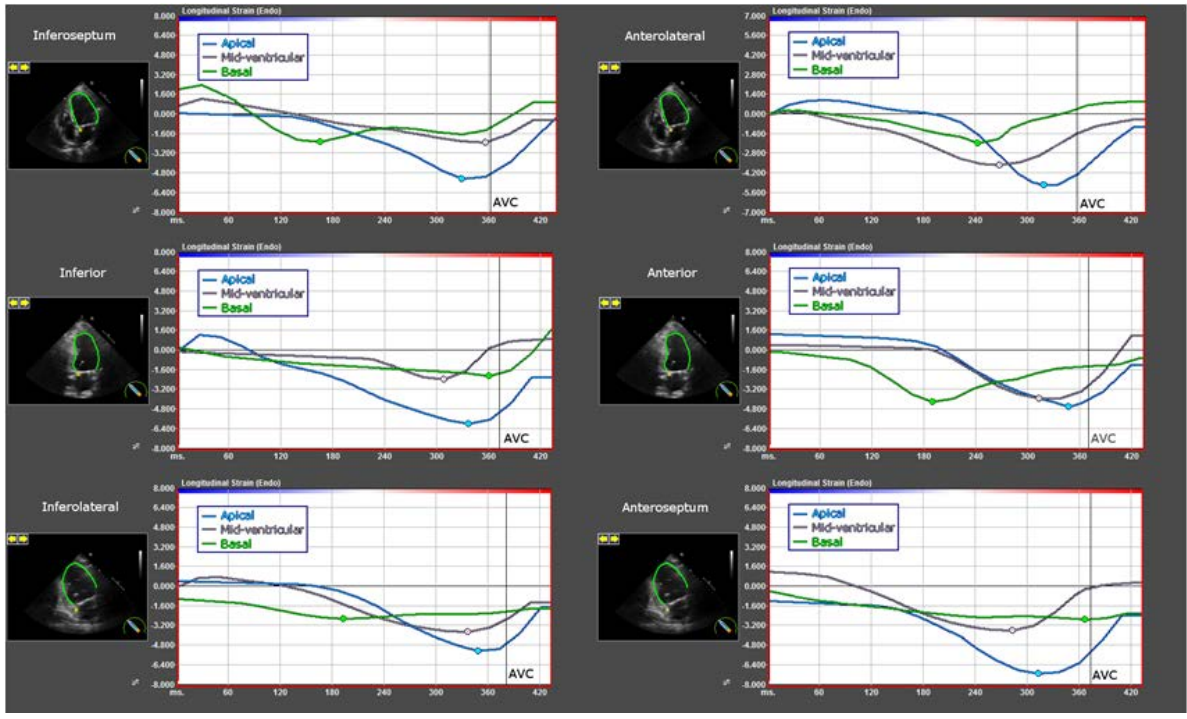

Supplemental Figure 3. Postoperative segmental longitudinal strain in patient with preoperative symmetric tethering (the same patient in Supplemental Figure 2). Peak strain worsened at follow up in the inferoseptum, inferior and antero-septum at the basal and mid-levels, remaining unchanged in the others segments. AVC: Aortic Valve Closure.

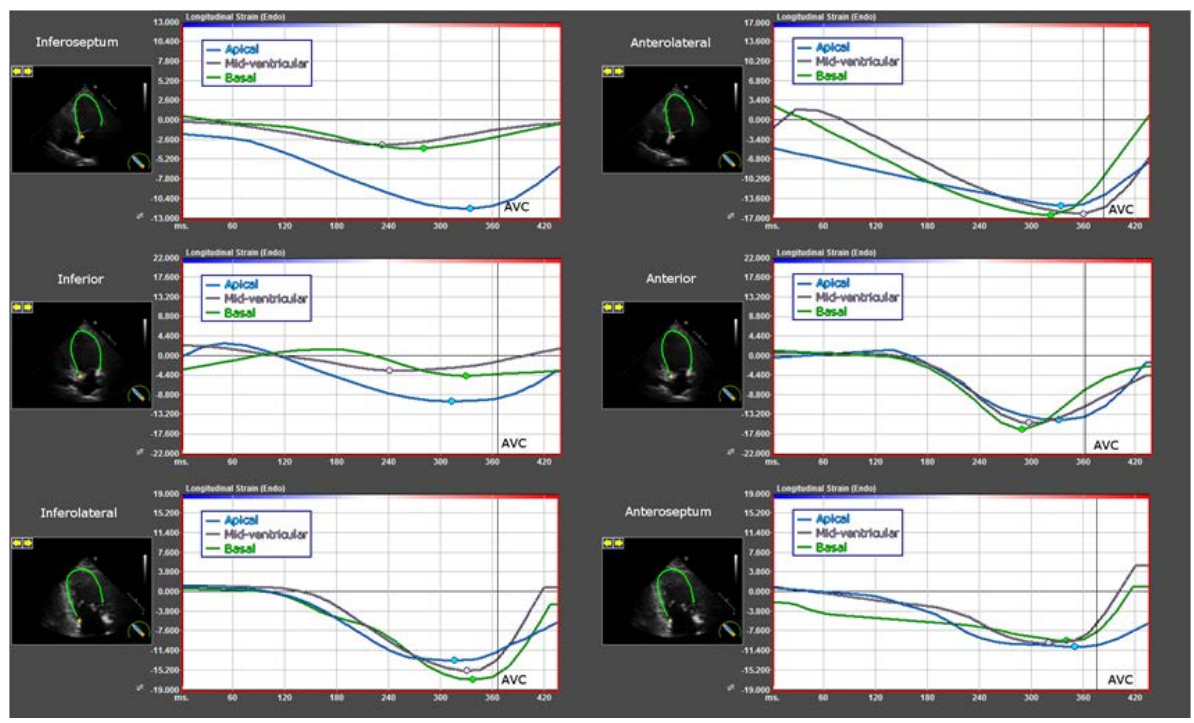

Supplemental Figure 4. Baseline segmental longitudinal strain in a patient with preoperative asymmetric tethering. Peak strain was worse at the levels of the basal and mid-ventricular segments in the inferior and infero-septal walls. AVC: Aortic Valve Closure. 


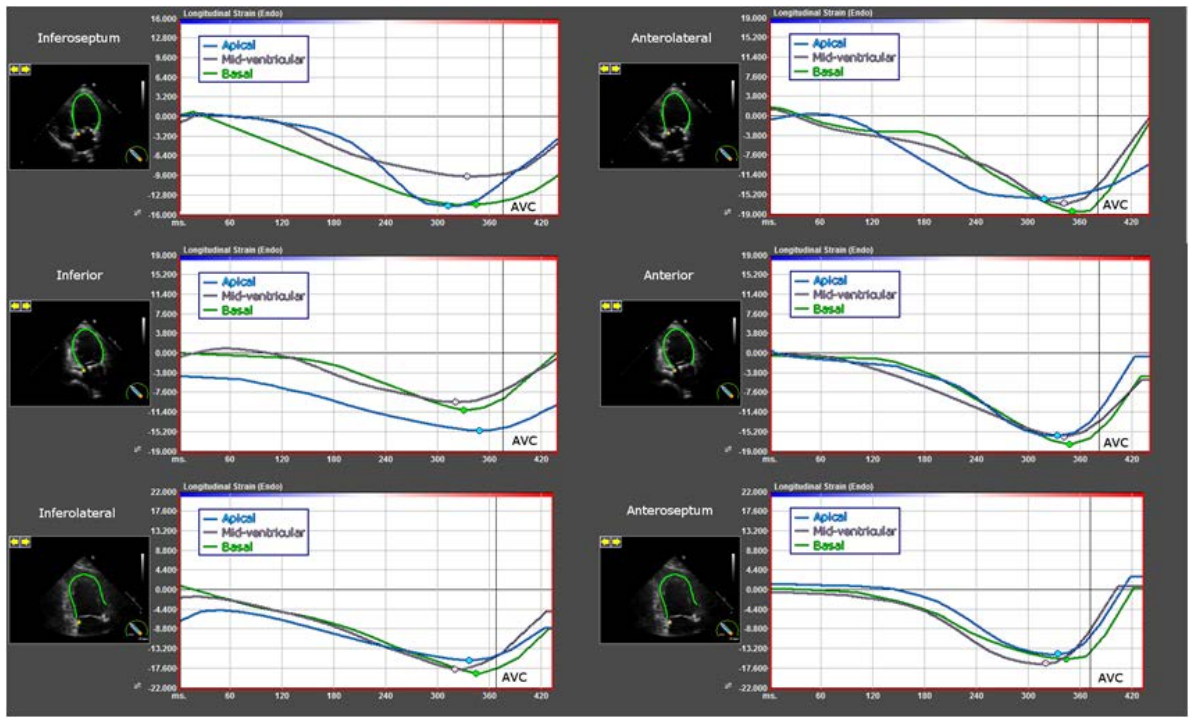

Supplemental Figure 5. Postoperative segmental longitudinal strain in a patient with preoperative asymmetric tethering (the same patient in Supplemental Figure 4). Peak strain showed a significant improvement in all segments. AVC: Aortic Valve Closure 


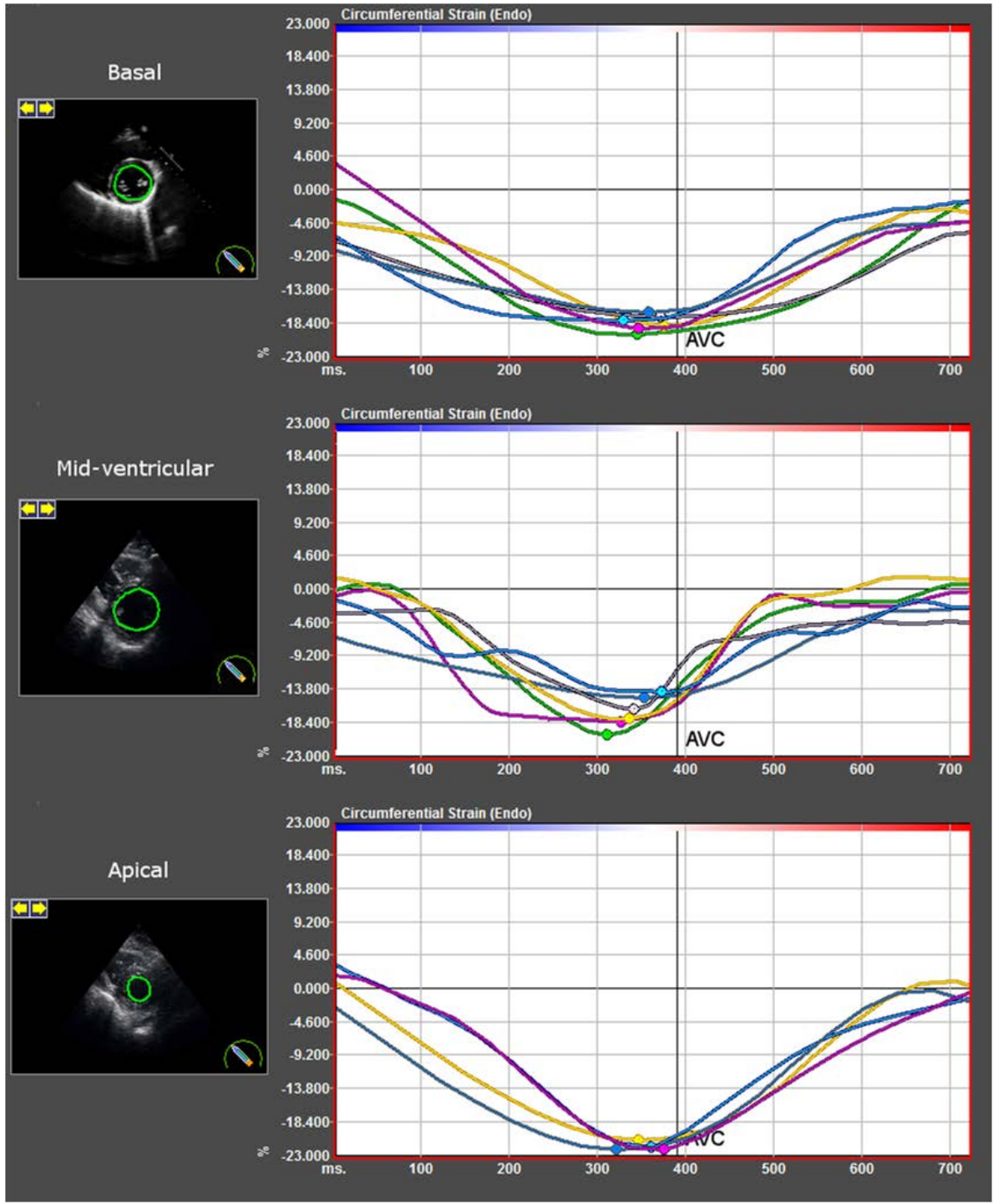

Supplemental Figure 6. Segmental circumferential s strain in a healthy control. 


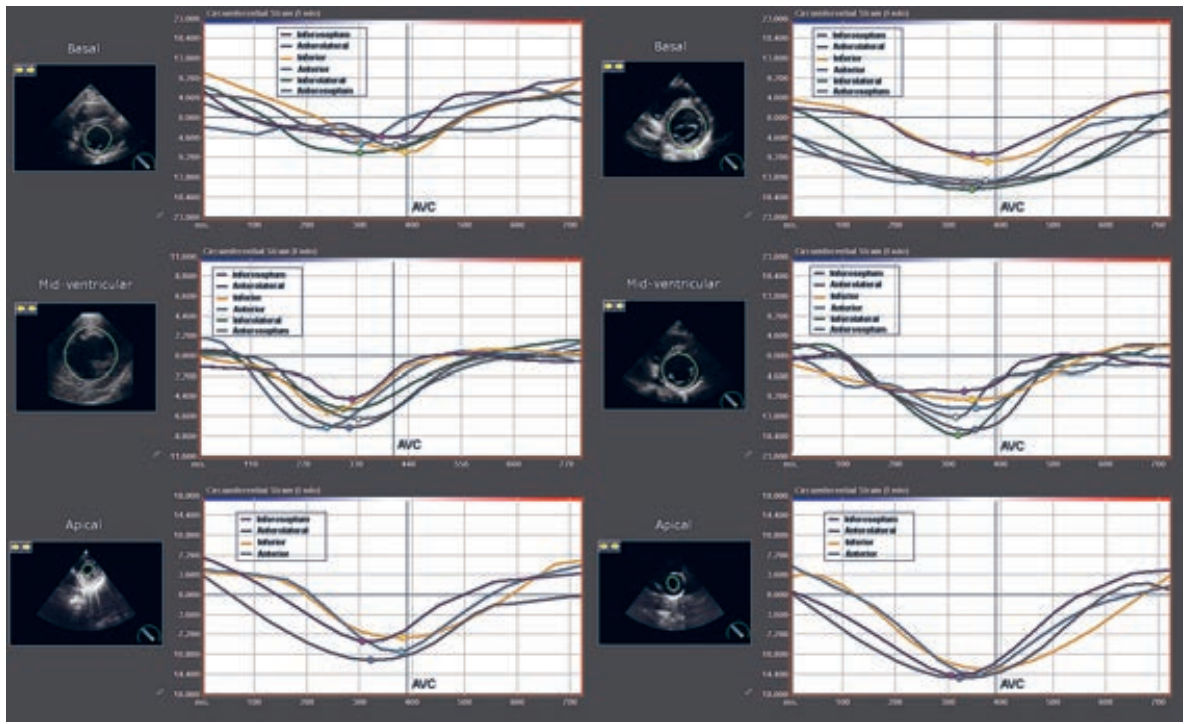

Supplemental Figure 7. Baseline segmental circumferential strain in a patient with preoperative symmetric tethering (left) and one with asymmetric tethering (right) in basal, mid and apical short-axis views. Strain was higher in the symmetric patient in all segments. In contrast, the patients with asymmetric tethering showed higher circumferential strain values in the inferoseptal and inferior walls at basal and midventricular levels. AVC: Aortic Valve Closure

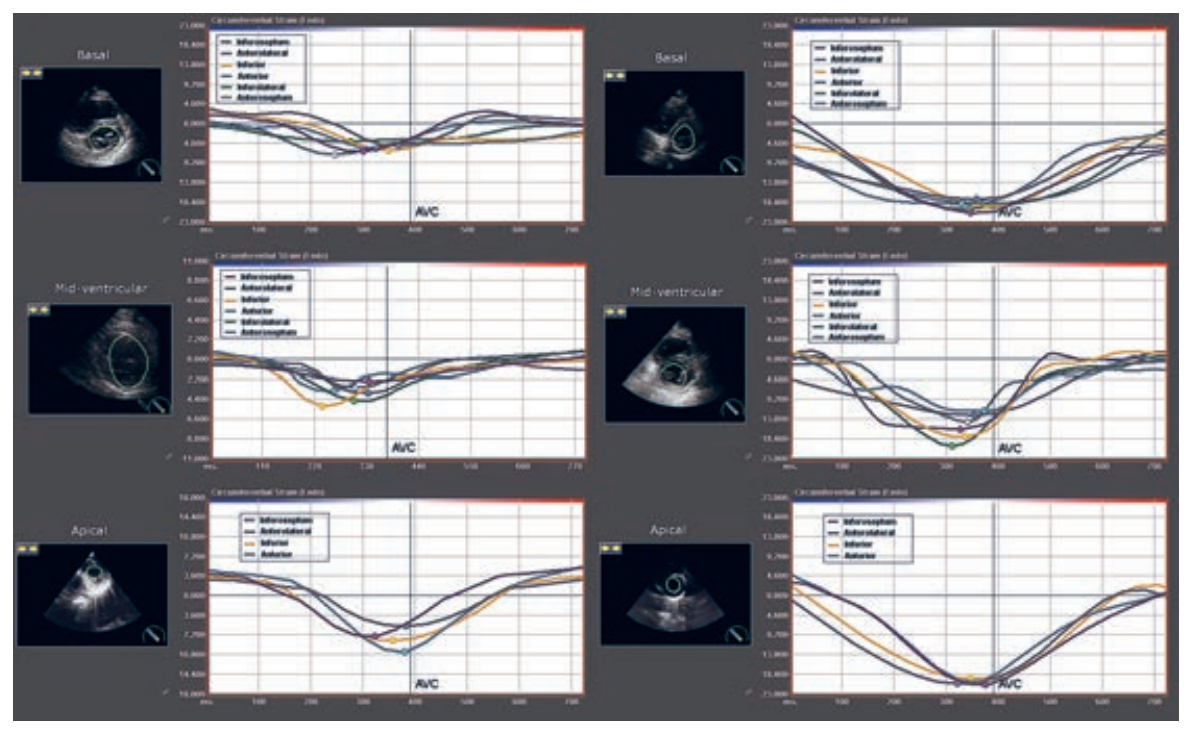

Supplemental Figure 8. Segmental circumferential strain in a patient with preoperative symmetric tethering (the same patient in Supplemental Figure 2, left) and one with asymmetric tethering (the same patient in Supplemental Figure 4, right) in basal, mid and apical short-axis views. In the patient belonging to Group 2 (right) circumferential strain improved in all segments whereas in the symmetric patient (left) it worsened most of the segments. AVC: Aortic Valve Closure. 


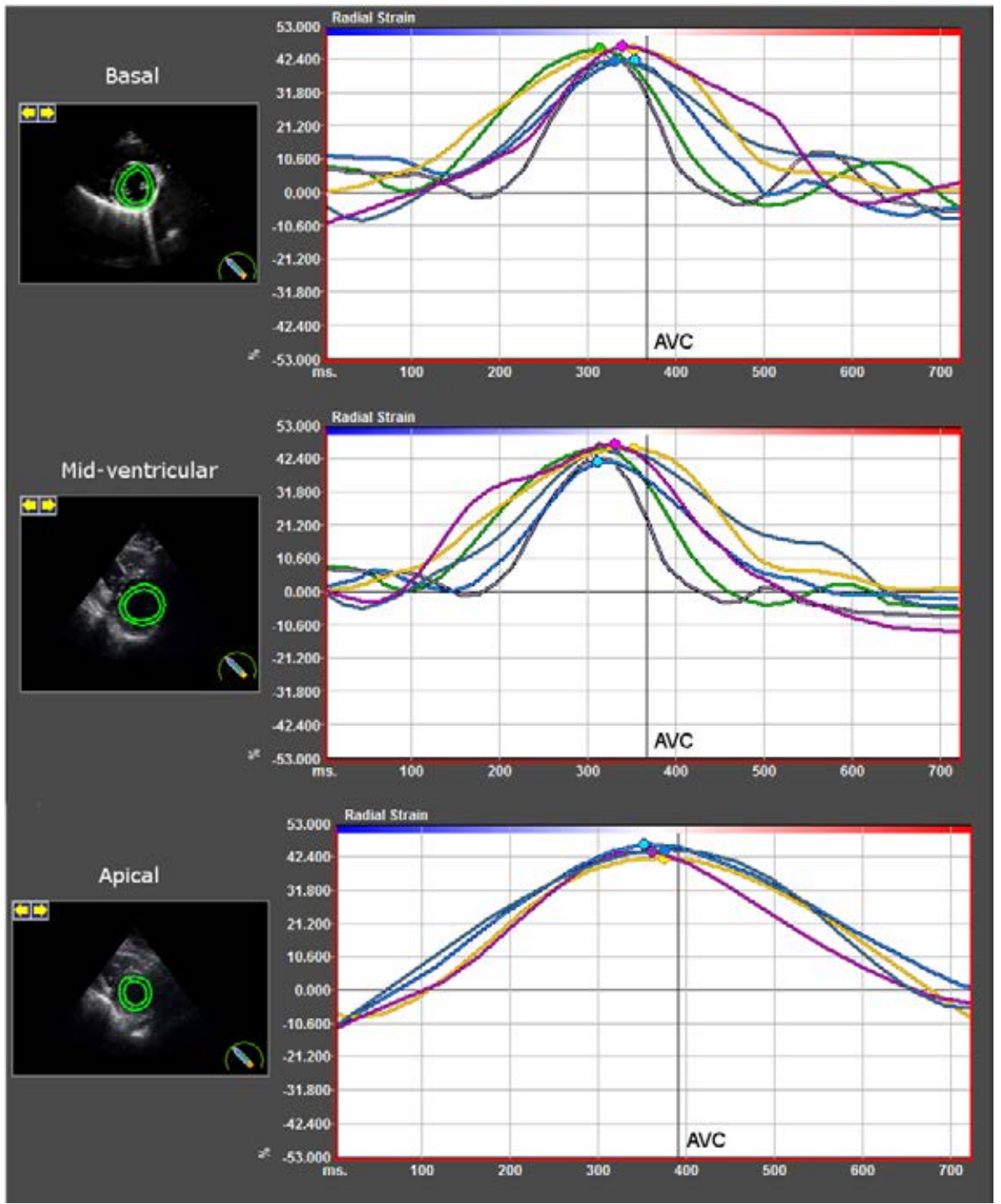

Supplemental Figure 9. Segmental radial strain in a healthy control. 


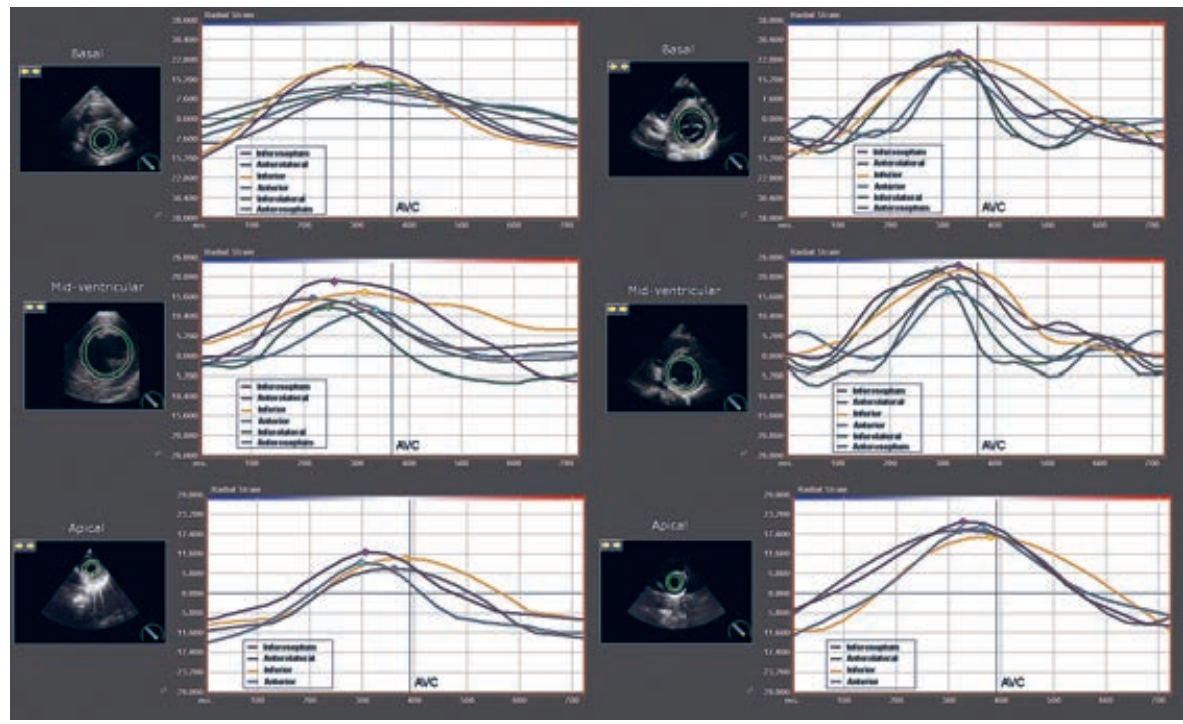

Supplemental Figure 10. Baseline segmental radial strain in a patient with preoperative symmetric tethering (left) and one with asymmetric tethering (right) in the basal, mid and apical short-axis views. Strain was lower in the symmetric patient in all segments in the apical view and in the anterolateral, anterior, inferolateral and anteroseptal segments in the mid-ventricular and basal walls. AVC: Aortic Valve Closure

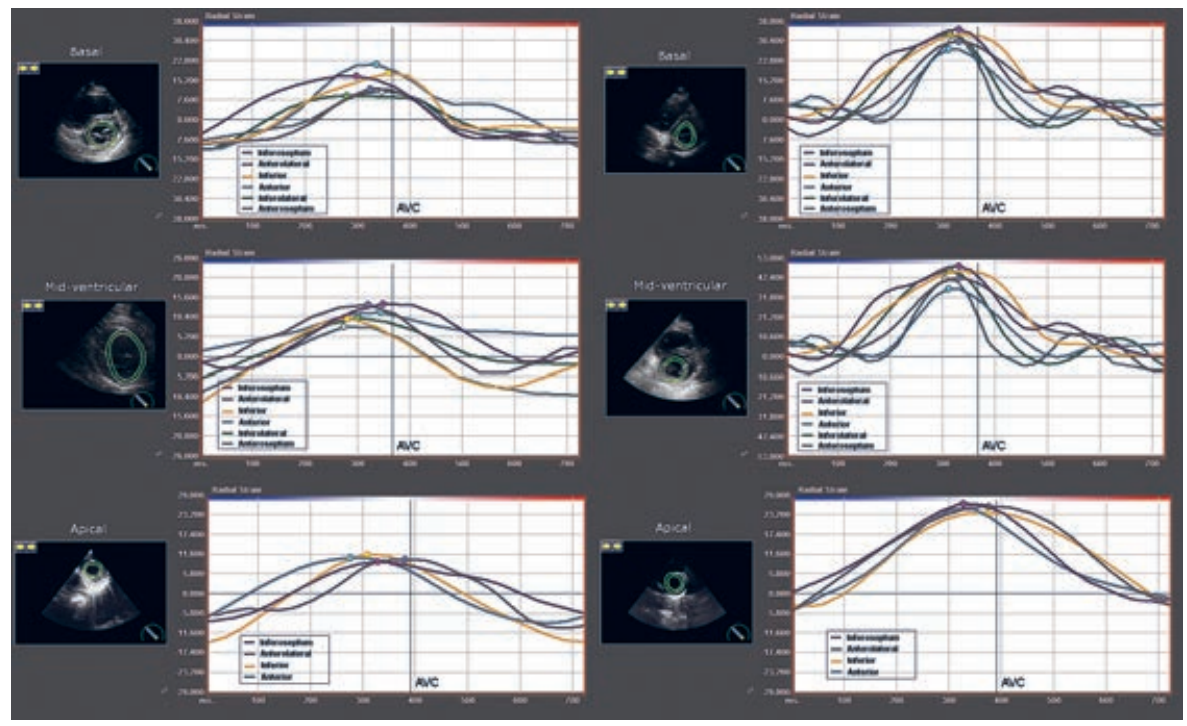

Supplemental Figure 11. Follow-up segmental radial strain in a patient with preoperative symmetric tethering (the same patient in Supplemental Figure 10, left) and one with asymmetric tethering (the same patient in Supplemental Figure 10, right) in the basal, mid and apical short-axis views. At follow up, radial systolic strain worsened, in the symmetric patient, in the inferior and inferoseptal segments. In contrast, strain improved in all segments in the symmetric patient. AVC: Aortic Valve Closure. 


\section{Appendix 2: Supplemental tables}

Supplemental Table 1. Baseline Segmental Longitudinal Systolic Strain

\begin{tabular}{|c|c|c|c|c|c|c|c|c|}
\hline & \multicolumn{4}{|c|}{ Infero-septum } & \multicolumn{4}{|c|}{ Anterolateral } \\
\hline & Controls & Symmetric & Asymmetric & $p$ & Controls & Symmetric & Asymmetric & $\mathrm{p}$ \\
\hline Apical & $-18.2 \pm 3.6$ & $-6.0 \pm 3.0^{*}$ & $-11.1 \pm 3.4^{*}$ & $<0.001$ & $-16.4 \pm 3.7$ & $-5.2 \pm 3.3^{*}$ & $-14.2 \pm 3.5$ & $<0.001$ \\
\hline Mid-ventricular & $-18.3 \pm 3.5$ & $-4.2 \pm 3.2^{*}$ & $-3.1 \pm 2.2^{*}$ & 0.3 & $-17.0 \pm 4.1$ & $-2.3 \pm 2.7^{*}$ & $-16.0 \pm 3.2$ & $<0.001$ \\
\hline \multirow[t]{3}{*}{ Basal } & $-15.1 \pm 2.9$ & $-4.5 \pm 2.0^{*}$ & $-3.4 \pm 2.4^{*}$ & 0.2 & $-20.0 \pm 4.3$ & $-3.3 \pm 2.1^{*}$ & $-17.1 \pm 3.4$ & $<0.001$ \\
\hline & \multicolumn{4}{|c|}{ Inferior } & \multicolumn{4}{|c|}{ Anterior } \\
\hline & Controls & Symmetric & Asymmetric & $\mathrm{p}$ & Controls & Symmetric & Asymmetric & $p$ \\
\hline Apical & $-17.9 \pm 3.3$ & $-6.2 \pm 3.3^{*}$ & $-9.0 \pm 3.3^{*}$ & 0.08 & $-16.0 \pm 3.0$ & $-5.1 \pm 2.5^{*}$ & $-14.2 \pm 3.4$ & $<0.001$ \\
\hline Mid-ventricular & $-17.0 \pm 3.1$ & $-4.2 \pm 3.1^{*}$ & $-3.2 \pm 2.6^{*}$ & 0.1 & $-17.9 \pm 3.6$ & $-3.4 \pm 2.7^{*}$ & $-16.3 \pm 3.1$ & $<0.001$ \\
\hline \multirow[t]{3}{*}{ Basal } & $-16.1 \pm 3.3$ & $-4.7 \pm 2.8^{*}$ & $-4.2 \pm 2.9^{*}$ & 0.6 & $-18.4 \pm 4.0$ & $-3.9 \pm 2.5^{*}$ & $-17.0 \pm 3.3$ & $<0.001$ \\
\hline & \multicolumn{4}{|c|}{ Inferolateral } & \multicolumn{4}{|c|}{ Antero-septum } \\
\hline & Controls & Symmetric & Asymmetric & $\mathrm{p}$ & Controls & Symmetric & Asymmetric & $\mathrm{p}$ \\
\hline Apical & $-16.9 \pm 3.9$ & $-5.0 \pm 3.4^{*}$ & $-14.4 \pm 4.4$ & $<0.001$ & $-17.8 \pm 3.5$ & $-6.3 \pm 3.3^{*}$ & $-11.4 \pm 2.8^{*}$ & 0.002 \\
\hline Mid-ventricular & $-17.5 \pm 4.0$ & $-3.3 \pm 2.9^{*}$ & $-16.2 \pm 3.8$ & $<0.001$ & $-18.0 \pm 3.7$ & $-6.0 \pm 3.4^{*}$ & $-11.3 \pm 3.7^{*}$ & 0.01 \\
\hline Basal & $-19.2 \pm 3.8$ & $-3.5 \pm 2.0^{*}$ & $-16.9 \pm 4.0$ & $<0.001$ & $-14.6 \pm 2.6$ & $-6.0 \pm 2.7^{*}$ & $-11.6 \pm 3.5^{*}$ & 0.03 \\
\hline
\end{tabular}

* $p<0.05$ vs. controls; $p=$ significance symmetric vs. asymmetric.

Supplemental Table 2. Segmental Longitudinal Systolic Strain at Follow up

\begin{tabular}{|c|c|c|c|c|c|c|c|c|}
\hline & \multicolumn{4}{|c|}{ Infero-septum } & \multicolumn{4}{|c|}{ Anterolateral } \\
\hline & Controls & Symmetric & Asymmetric & $p$ & Controls & Symmetric & Asymmetric & $p$ \\
\hline Apical & $-18.2 \pm 3.6$ & $-6.1 \pm 2.9^{*}$ & $-15.1 \pm 4.4^{*+}$ & $<0.001$ & $-16.4 \pm 3.7$ & $-5.3 \pm 3.5^{*}$ & $-16.5 \pm 3.5^{\dagger}$ & $<0.001$ \\
\hline Mid-ventricular & $-18.3 \pm 3.5$ & $-2.2 \pm 2.5^{*+}$ & $-9.9 \pm 3.6^{*+}$ & $<0.001$ & $-17.0 \pm 4.1$ & $-3.5 \pm 2.5^{*}$ & $-17.4 \pm 4.0^{+}$ & $<0.001$ \\
\hline \multirow[t]{3}{*}{ Basal } & $-15.1 \pm 2.9$ & $-2.0 \pm 1.9^{*+}$ & $-14.9 \pm 3.2^{+}$ & $<0.001$ & $-20.0 \pm 4.3$ & $-2.5 \pm 2.0^{*}$ & $-19.8 \pm 4.1^{\dagger}$ & $<0.001$ \\
\hline & \multicolumn{4}{|c|}{ Inferior } & \multicolumn{4}{|c|}{ Anterior } \\
\hline & Controls & Symmetric & Asymmetric & $\mathrm{p}$ & Controls & Symmetric & Asymmetric & $\mathrm{p}$ \\
\hline Apical & $-17.9 \pm 3.3$ & $-5.8 \pm 3.0^{*}$ & $-15.5 \pm 3.4^{*+}$ & $<0.001$ & $-16.0 \pm 3.0$ & $-5.0 \pm 2.3^{*}$ & $-16.0 \pm 3.7^{+}$ & $<0.001$ \\
\hline Mid-ventricular & $-17.0 \pm 3.1$ & $-2.2 \pm 2.0^{*+}$ & $-9.7 \pm 3.9^{* \dagger}$ & $<0.001$ & $-17.9 \pm 3.6$ & $-3.8 \pm 2.9^{*}$ & $-17.7 \pm 4.5^{\dagger}$ & $<0.001$ \\
\hline \multirow[t]{3}{*}{ Basal } & $-16.1 \pm 3.3$ & $-1.8 \pm 1.8^{*+}$ & $-11.0 \pm 4.8^{* \dagger}$ & $<0.001$ & $-18.4 \pm 4.0$ & $-4.1 \pm 2.9^{*}$ & $-18.4 \pm 4.9^{+}$ & $<0.001$ \\
\hline & \multicolumn{4}{|c|}{ Inferolateral } & \multicolumn{4}{|c|}{ Antero-septum } \\
\hline & Controls & Symmetric & Asymmetric & $p$ & Controls & Symmetric & Asymmetric & $p$ \\
\hline Apical & $-16.9 \pm 3.9$ & $-5.2 \pm 3.3^{*}$ & $-16.5 \pm 3.5^{\dagger}$ & $<0.001$ & $-17.8 \pm 3.5$ & $-6.7 \pm 3.2^{*}$ & $-15.1 \pm 3.8^{\dagger}$ & $<0.001$ \\
\hline Mid-ventricular & $-17.5 \pm 4.0$ & $-3.6 \pm 2.5^{*}$ & $-17.3 \pm 3.9^{+}$ & $<0.001$ & $-18.0 \pm 3.7$ & $-3.3 \pm 2.4^{*+}$ & $-16.6 \pm 3.6^{\dagger}$ & $<0.001$ \\
\hline Basal & $-19.2 \pm 3.8$ & $-2.9 \pm 2.0^{*}$ & $-18.6 \pm 3.7^{\dagger}$ & $<0.001$ & $-14.6 \pm 2.6$ & $-3.0 \pm 2.0^{*+}$ & $-15.8 \pm 3.5^{+}$ & $<0.001$ \\
\hline
\end{tabular}

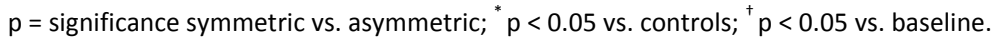


Supplemental Table 3. Segmental Circumferential Systolic Strain

\begin{tabular}{|c|c|c|c|c|c|c|c|}
\hline & & & Baseline & & & Eollow-up & \\
\hline \multicolumn{8}{|l|}{ Basal } \\
\hline & Controls & Symmetric & Asymmetric & $\mathrm{p}$ & Symmetric & Asymmetric & $\mathrm{p}$ \\
\hline Infero-septum & $-24.1 \pm 6.3$ & $-7.0 \pm 3.1^{*}$ & $-9.4 \pm 3.2^{*}$ & 0.51 & $-5.3 \pm 2.7^{* \dagger}$ & $-18.4 \pm 5.5^{* \dagger}$ & $<0.001$ \\
\hline Anterolateral & $-18.1 \pm 5.2$ & $-5.2 \pm 2.2^{*}$ & $-15.1 \pm 3.9^{*}$ & $<0.001$ & $-4.8 \pm 2.3^{*}$ & $-17.5 \pm 4.3^{* \dagger}$ & $<0.001$ \\
\hline Inferior & $-20.1 \pm 5.3$ & $-7.1 \pm 3.9^{*}$ & $-7.9 \pm 4.1^{*}$ & 0.9 & $-5.4 \pm 3.5^{*+}$ & $-16.3 \pm 4.0^{* \dagger}$ & $<0.001$ \\
\hline Anterior & $-22.1 \pm 5.4$ & $-5.4 \pm 2.3^{*}$ & $-14.3 \pm 4.4^{*}$ & $<0.001$ & $-5.3 \pm 2.5^{*}$ & $-18.8 \pm 4.9^{* \dagger}$ & $<0.001$ \\
\hline Inferolateral & $-20.3 \pm 5.6$ & $-5.7 \pm 2.4^{*}$ & $-17.0 \pm 4.0^{*}$ & $<0.001$ & $5.5 \pm 2.5^{*}$ & $-19.4 \pm 5.3^{*+}$ & $<0.001$ \\
\hline Antero-septum & $-25.1 \pm 6.1$ & $-5.8 \pm 2.5^{*}$ & $-14.7 \pm 4.4^{*}$ & $<0.001$ & $4.4 \pm 2.1^{*+}$ & $-20.3 \pm 5.7^{*+}$ & $<0.001$ \\
\hline \multicolumn{8}{|l|}{ Mid-ventricular } \\
\hline & Controls & Symmetric & Asymmetric & $\mathrm{p}$ & Symmetric & Asymmetric & $\mathrm{p}$ \\
\hline Infero-septum & $-23.6 \pm 6.1$ & $-6.9 \pm 3.8^{*}$ & $-7.2 \pm 3.9^{*}$ & 0.73 & $-3.5 \pm 2.9^{* \dagger}$ & $-16.5 \pm 4.0^{*+}$ & $<0.001$ \\
\hline Anterolateral & $-20.7 \pm 5.9$ & $-3.1 \pm 2.9^{*}$ & $-13.7 \pm 4.7$ & $<0.001$ & $-3.1 \pm 3.2^{*}$ & $-18.1 \pm 4.2^{* \dagger}$ & $<0.001$ \\
\hline Inferior & $-19.6 \pm 4.4$ & $-5.2 \pm 3.6^{*}$ & $-6.8 \pm 3.7^{*}$ & 0.84 & $-2.4 \pm 2.0^{* \dagger}$ & $-16.9 \pm 3.9^{* \dagger}$ & $<0.001$ \\
\hline Anterior & $-20.9 \pm 5.6$ & $-5.2 \pm 2.6^{*}$ & $-15.6 \pm 5.1^{*}$ & $<0.001$ & $-4.6 \pm 2.1^{*}$ & $-19.0 \pm 3.8^{* \dagger}$ & $<0.001$ \\
\hline Inferolateral & $-19.7 \pm 5.2$ & $-3.0 \pm 2.2^{*}$ & $-16.9 \pm 4.0^{*}$ & $<0.001$ & $-2.8 \pm 2.3^{*}$ & $-18.5 \pm 4.1^{* \dagger}$ & $<0.001$ \\
\hline Antero-septum & $-21.4 \pm 5.3$ & $-5.1 \pm 2.7^{*}$ & $-13.1 \pm 4.4^{*}$ & $<0.001$ & $-3.1 \pm 1.9^{* \dagger}$ & $-19.1 \pm 4.0^{* \dagger}$ & $<0.001$ \\
\hline \multicolumn{8}{|l|}{ Apical } \\
\hline & Controls & Symmetric & Asymmetric & $\mathrm{p}$ & Symmetric & Asymmetric & $p$ \\
\hline Infero-septum & $-25.8 \pm 5.0$ & $-7.3 \pm 2.9^{*}$ & $-15.2 \pm 4.7^{*}$ & 0.03 & $-5.9 \pm 3.4^{* \dagger}$ & $-19.7 \pm 5.9^{* \dagger}$ & $<0.001$ \\
\hline Anterolateral & $-26.1 \pm 5.9$ & $-8.1 \pm 3.0^{*}$ & $-15.7 \pm 4.8^{*}$ & $<0.001$ & $-8.3 \pm 3.1^{*}$ & $-19.9 \pm 5.4^{* \dagger}$ & $<0.001$ \\
\hline Inferior & $-24.9 \pm 5.8$ & $-11.7 \pm 4.4^{*}$ & $-12.5 \pm 3.9^{*}$ & 0.69 & $-11.0 \pm 4.2^{*}$ & $-20.1 \pm 5.3^{*+}$ & $<0.001$ \\
\hline Anterior & $-25.1 \pm 5.7$ & $-9.9 \pm 3.1^{*}$ & $-14.5 \pm 4.4^{*}$ & $<0.001$ & $-9.4 \pm 2.8^{*}$ & $-21.1 \pm 5.1^{* \dagger}$ & $<0.001$ \\
\hline
\end{tabular}

$p=$ significance symmetric vs. asymmetric; $* p<0.05$ vs. controls; $\uparrow p<0.05$ vs. baseline. 
Supplemental Table 4. Segmental Radial Systolic Strain

\begin{tabular}{|c|c|c|c|c|c|c|c|}
\hline & & & Baseline & & & Follow-up & \\
\hline \multicolumn{8}{|l|}{ Basal } \\
\hline & Controls & Symmetric & Asymmetric & $\mathrm{p}$ & Symmetric & Asymmetric & $\mathrm{p}$ \\
\hline Infero-septum & $42.0 \pm 11.7$ & $19.3 \pm 8.2^{*}$ & $18.8 \pm 7.7^{*}$ & 0.55 & $20.0 \pm 9.4^{*}$ & $32.5 \pm 5.4^{*+}$ & $<0.001$ \\
\hline Anterolateral & $45.2 \pm 14.3$ & $10.0 \pm 6.0^{*}$ & $22.2 \pm 11.8^{*}$ & $<0.001$ & $10.4 \pm 6.1^{*}$ & $31.1 \pm 9.9^{*+}$ & $<0.001$ \\
\hline Inferior & $45.7 \pm 15.2$ & $15.9 \pm 8.1^{*}$ & $16.0 \pm 8.4^{*}$ & 0.9 & $8.8 \pm 5.4^{* \dagger}$ & $32.7 \pm 10.9^{* \dagger}$ & $<0.001$ \\
\hline Anterior & $44.1 \pm 14.6$ & $7.3 \pm 4.1^{*}$ & $18.7 \pm 7.4^{*}$ & $<0.001$ & $7.7 \pm 4.6^{*}$ & $34.4 \pm 10.2^{*+}$ & $<0.001$ \\
\hline Inferolateral & $46.3 \pm 12.1$ & $8.0 \pm 4.1^{*}$ & $22.0 \pm 11.5^{*}$ & $<0.001$ & $8.4 \pm 4.7^{*}$ & $31.8 \pm 10.6^{*+}$ & $<0.001$ \\
\hline Antero-septum & $44.0 \pm 14.5$ & $10.4 \pm 8.4^{*}$ & $22.2 \pm 9.9^{*}$ & $<0.001$ & $6.9 \pm 3.8^{*+}$ & $34.0 \pm 9.1^{* \dagger}$ & $<0.001$ \\
\hline \multicolumn{8}{|l|}{ Mid-ventricular } \\
\hline & Controls & Symmetric & Asymmetric & $\mathrm{p}$ & Symmetric & Asymmetric & $\mathrm{p}$ \\
\hline Infero-septum & $44.3 \pm 12.2$ & $14.4 \pm 9.1^{*}$ & $15.6 \pm 8.8^{*}$ & 0.75 & $13.9 \pm 8.8^{*}$ & $33.5 \pm 9.2^{* \dagger}$ & $<0.001$ \\
\hline Anterolateral & $48.4 \pm 15.4$ & $8.8 \pm 5.2^{*}$ & $20.5 \pm 12^{*}$ & $<0.001$ & $9.3 \pm 5.2^{*}$ & $34.7 \pm 11.6^{* \dagger}$ & $<0.001$ \\
\hline Inferior & $50.1 \pm 14.1$ & $13.9 \pm 7.8^{*}$ & $13.4 \pm 9.4^{*}$ & 0.9 & $9.3 \pm 5.6^{*+}$ & $34.4 \pm 12.3^{*+}$ & $<0.001$ \\
\hline Anterior & $47.7 \pm 15.7$ & $6.4 \pm 5.9^{*}$ & $17.8 \pm 9.9^{*}$ & $<0.001$ & $6.5 \pm 5.6^{*}$ & $37.9 \pm 12.4^{* \dagger}$ & $<0.001$ \\
\hline Inferolateral & $49.2 \pm 15.9$ & $6.8 \pm 5.6^{*}$ & $19.0 \pm 8.6^{*}$ & $<0.001$ & $6.4 \pm 3.7^{*}$ & $34.9 \pm 11.4^{* \dagger}$ & $<0.001$ \\
\hline Antero-septum & $43.1 \pm 12.6$ & $9.4 \pm 5.4^{*}$ & $20.8 \pm 9.3^{*}$ & $<0.001$ & $6.8 \pm 3.8^{*+}$ & $36.0 \pm 9.8^{*+}$ & $<0.001$ \\
\hline \multicolumn{8}{|l|}{ Apical } \\
\hline & Controls & Symmetric & Asymmetric & $\mathrm{p}$ & Symmetric & Asymmetric & $p$ \\
\hline Infero-septum & $35.7 \pm 11.3$ & $9.3 \pm 5.0^{*}$ & $18.3 \pm 10.3^{*}$ & $<0.001$ & $10.6 \pm 5.4^{*}$ & $29.9 \pm 8.8^{*+}$ & $<0.001$ \\
\hline Anterolateral & $35.9 \pm 12.7$ & $9.3 \pm 4.8^{*}$ & $22.4 \pm 10.2^{*}$ & $<0.001$ & $10.2 \pm 5.0^{*}$ & $30.4 \pm 5.9^{*+}$ & $<0.001$ \\
\hline Inferior & $35.8 \pm 12.9$ & $14.4 \pm 7.8^{*}$ & $16.2 \pm 8.4^{*}$ & 0.007 & $12.8 \pm 6.6^{*}$ & $25.8 \pm 10.1^{* \dagger}$ & $<0.001$ \\
\hline Anterior & $36.0 \pm 13.1$ & $10.1 \pm 4.4^{*}$ & $20.3 \pm 10.9^{*}$ & $<0.001$ & $9.8 \pm 5.3^{*}$ & $30.0 \pm 8.8^{* \dagger}$ & $<0.001$ \\
\hline
\end{tabular}

$\mathrm{p}=$ significance symmetric vs. asymmetric; $* p<0.05$ vs. controls; $\uparrow p<0.05$ vs. baseline. 


\section{References}

1. Edvardsen $T$, Skulstad H, Aakhus S, Urheim S, Ihlen H. Regional myocardial systolic function during acute myocardial ischemia assessed by strain doppler echocardiography. Journal of the American College of Cardiology. 2001;37:726-730

2. Tsai WC, Liu YW, Huang YY, Lin CC, Lee CH, Tsai LM. Diagnostic value of segmental longitudinal strain by automated function imaging in coronary artery disease without left ventricular dysfunction. Journal of the American Society of Echocardiography: official publication of the American Society of Echocardiography. 2010;23:1183-1189

3. Vartdal T, Brunvand H, Pettersen E, Smith HJ, Lyseggen E, Helle-Valle T, Skulstad H, Ihlen H, Edvardsen T. Early prediction of infarct size by strain doppler echocardiography after coronary reperfusion. Journal of the American College of Cardiology. 2007;49:1715-1721

4. Sjoli B, Orn S, Grenne B, Vartdal T, Smiseth OA, Edvardsen T, Brunvand H. Comparison of left ventricular ejection fraction and left ventricular global strain as determinants of infarct size in patients with acute myocardial infarction. Journal of the American Society of Echocardiography: official publication of the American Society of Echocardiography. 2009;22:1232-1238

5. Nesser HJ, Winter S. Speckle tracking in the evaluation of left ventricular dyssynchrony. Echocardiography. 2009;26:324-336

6. Gjesdal O, Vartdal T, Hopp E, Lunde K, Brunvand H, Smith HJ, Edvardsen T. Left ventricle longitudinal deformation assessment by mitral annulus displacement or global longitudinal strain in chronic ischemic heart disease: Are they interchangeable? Journal of the American Society of Echocardiography: official publication of the American Society of Echocardiography. 2009;22:823-830

7. de Isla LP, de Agustin A, Rodrigo JL, Almeria C, del Carmen Manzano M, Rodriguez E, Garcia A, Macaya C, Zamorano J. Chronic mitral regurgitation: A pilot study to assess preoperative left ventricular contractile function using speckle-tracking echocardiography. Journal of the American Society of Echocardiography: official publication of the American Society of Echocardiography. 2009;22:831-838

8. Eek C, Grenne B, Brunvand H, Aakhus S, Endresen K, Smiseth OA, Edvardsen T, Skulstad H. Strain echocardiography predicts acute coronary occlusion in patients with non-st-segment elevation acute coronary syndrome. European journal of echocardiography: the journal of the Working Group on Echocardiography of the European Society of Cardiology. 2010;11:501-508

9. Helin LM, Tamas E, Nylander E. Preoperative longitudinal left ventricular function by tissue doppler echocardiography at rest and during exercise is valuable in timing of aortic valve surgery in male aortic regurgitation patients. Journal of the American Society of Echocardiography: official publication of the American Society of Echocardiography. 2010;23:387-395

10. Gelsomino S, Van Garsse L, Luca F, Lorusso R, Cheriex E, Rao CM, Caciolli S, Vizzardi E, Crudeli E, Stefano $P$, Gensini GF, Maessen J. Impact of preoperative anterior leaflet tethering on the recurrence of ischemic mitral regurgitation and the lack of left ventricular reverse remodeling after restrictive annuloplasty. Journal of the American Society of Echocardiography: official publication of the American Society of Echocardiography. 2011;24:1365-1375

11. Gelsomino S, Lorusso R, De Cicco G, Bille G, Caciolli S, Rostagno C, Capecchi I, Chioccioli M, Stefano P, Gensini GF. Does preoperative tethering symmetry affect left ventricular reverse remodeling after restrictive annuloplasty? International journal of cardiology. 2010;141:182-191

12. Gelsomino S, Lorusso R, Caciolli S, Capecchi I, Rostagno C, Chioccioli M, De Cicco G, Bille G, Stefano P, Gensini GF. Insights on left ventricular and valvular mechanisms of recurrent ischemic mitral regurgitation after restrictive annuloplasty and coronary artery bypass grafting. The Journal of thoracic and cardiovascular surgery. 2008;136:507-518 
13. Zoghbi WA, Enriquez-Sarano M, Foster E, Grayburn PA, Kraft CD, Levine RA, Nihoyannopoulos $P$, Otto CM, Quinones MA, Rakowski H, Stewart WJ, Waggoner A, Weissman NJ. Recommendations for evaluation of the severity of native valvular regurgitation with two-dimensional and doppler echocardiography. Journal of the American Society of Echocardiography: official publication of the American Society of Echocardiography. 2003;16:777-802

14. Lang RM, Bierig M, Devereux RB, Flachskampf FA, Foster E, Pellikka PA, Picard MH, Roman MJ, Seward J, Shanewise JS, Solomon SD, Spencer KT, Sutton MS, Stewart WJ. Recommendations for chamber quantification: A report from the american society of echocardiography's guidelines and standards committee and the chamber quantification writing group, developed in conjunction with the european association of echocardiography, a branch of the european society of cardiology. Journal of the American Society of Echocardiography: official publication of the American Society of Echocardiography. 2005;18:1440-1463

15. Cerqueira MD, Weissman NJ, Dilsizian V, Jacobs AK, Kaul S, Laskey WK, Pennell DJ, Rumberger JA, Ryan $T$, Verani MS. Standardized myocardial segmentation and nomenclature for tomographic imaging of the heart: A statement for healthcare professionals from the cardiac imaging committee of the council on clinical cardiology of the american heart association. Circulation. 2002;105:539-542

16. Grigioni F, Enriquez-Sarano M, Zehr KJ, Bailey KR, Tajik AJ. Ischemic mitral regurgitation: Long-term outcome and prognostic implications with quantitative doppler assessment. Circulation. 2001;103:1759-1764

17. Smedsrud MK, Pettersen E, Gjesdal O, Svennevig JL, Andersen K, Ihlen H, Edvardsen T. Detection of left ventricular dysfunction by global longitudinal systolic strain in patients with chronic aortic regurgitation. Journal of the American Society of Echocardiography: official publication of the American Society of Echocardiography. 2011;24:1253-1259

18. Sun JP, Lee AP, Wu C, Lam YY, Hung MJ, Chen L, Hu Z, Fang F, Yang XS, Merlino JD, Yu CM. Quantification of left ventricular regional myocardial function using two-dimensional speckle tracking echocardiography in healthy volunteers - a multi-center study. International journal of cardiology. 2012

19. Magne J, Mahjoub H, Pierard LA, O'Connor K, Pirlet C, Pibarot P, Lancellotti P. Prognostic importance of brain natriuretic peptide and left ventricular longitudinal function in asymptomatic degenerative mitral regurgitation. Heart. 2012;98:584-591

20. Marciniak A, Claus P, Sutherland GR, Marciniak M, Karu T, Baltabaeva A, Merli E, Bijnens B, Jahangiri M. Changes in systolic left ventricular function in isolated mitral regurgitation. A strain rate imaging study. European heart journal. 2007;28:2627-2636

21. Mascle S, Schnell F, Thebault C, Corbineau H, Laurent M, Hamonic S, Veillard D, Mabo P, Leguerrier A, Donal E. Predictive value of global longitudinal strain in a surgical population of organic mitral regurgitation. Journal of the American Society of Echocardiography: official publication of the American Society of Echocardiography. 2012;25:766-772

22. Lee R, Hanekom L, Marwick TH, Leano R, Wahi S. Prediction of subclinical left ventricular dysfunction with strain rate imaging in patients with asymptomatic severe mitral regurgitation. The American journal of cardiology. 2004;94:1333-1337

23. Kim MS, Kim YJ, Kim HK, Han JY, Chun HG, Kim HC, Sohn DW, Oh BH, Park YB. Evaluation of left ventricular short- and long-axis function in severe mitral regurgitation using 2-dimensional strain echocardiography. American heart journal. 2009;157:345-351

24. Carlhall CJ, Nguyen TC, Itoh A, Ennis DB, Bothe W, Liang D, Ingels NB, Miller DC. Alterations in transmural myocardial strain: An early marker of left ventricular dysfunction in mitral regurgitation? Circulation. 2008;118:S256-S262

25. Lancellotti P, Cosyns B, Zacharakis D, Attena E, Van Camp G, Gach O, Radermecker M, Pierard LA. Importance of left ventricular longitudinal function and functional reserve in patients with degenerative mitral regurgitation: Assessment by two-dimensional speckle tracking. Journal of the American Society of Echocardiography: official publication of the American Society of Echocardiography. 2008;21:1331-1336 
26. Lamas GA, Mitchell GF, Flaker GC, Smith SC, Jr., Gersh BJ, Basta L, Moye L, Braunwald E, Pfeffer MA. Clinical significance of mitral regurgitation after acute myocardial infarction. Survival and ventricular enlargement investigators. Circulation. 1997;96:827-833

27. Barzilai B, Gessler C, Jr., Perez JE, Schaab C, Jaffe AS. Significance of doppler-detected mitral regurgitation in acute myocardial infarction. The American journal of cardiology. 1988;61:220-223

28. Bax JJ, Braun J, Somer ST, Klautz R, Holman ER, Versteegh MI, Boersma E, Schalij MJ, van der Wall EE, Dion RA. Restrictive annuloplasty and coronary revascularization in ischemic mitral regurgitation results in reverse left ventricular remodeling. Circulation. 2004;110:II103-II108

29. McGee EC, Gillinov AM, Blackstone EH, Rajeswaran J, Cohen G, Najam F, Shiota T, Sabik JF, Lytle BW, McCarthy PM, Cosgrove DM. Recurrent mitral regurgitation after annuloplasty for functional ischemic mitral regurgitation. The Journal of thoracic and cardiovascular surgery. 2004;128:916-924

30. Hung J, Papakostas L, Tahta SA, Hardy BG, Bollen BA, Duran CM, Levine RA. Mechanism of recurrent ischemic mitral regurgitation after annuloplasty: Continued Iv remodeling as a moving target. Circulation. 2004;110:II85-II90

31. Agricola E, Oppizzi M, Maisano F, De Bonis M, Schinkel AF, Torracca L, Margonato A, Melisurgo G, Alfieri O. Echocardiographic classification of chronic ischemic mitral regurgitation caused by restricted motion according to tethering pattern. European journal of echocardiography: the journal of the Working Group on Echocardiography of the European Society of Cardiology. 2004;5:326-334

32. Godley RW, Wann LS, Rogers EW, Feigenbaum H, Weyman AE. Incomplete mitral leaflet closure in patients with papillary muscle dysfunction. Circulation. 1981;63:565-571

33. Borger MA, Alam A, Murphy PM, Doenst T, David TE. Chronic ischemic mitral regurgitation: Repair, replace or rethink? The Annals of thoracic surgery. 2006;81:1153-1161

34. Magne J, Pibarot P, Dumesnil JG, Senechal M. Continued global left ventricular remodeling is not the sole mechanism responsible for the late recurrence of ischemic mitral regurgitation after restrictive annuloplasty. Journal of the American Society of Echocardiography: official publication of the American Society of Echocardiography. 2009;22:1256-1264

35. Maltais S, Schaff HV, Daly RC, Suri RM, Dearani JA, Sundt TM, 3rd, Enriquez-Sarano M, Topilsky Y, Park SJ. Mitral regurgitation surgery in patients with ischemic cardiomyopathy and ischemic mitral regurgitation: Factors that influence survival. The Journal of thoracic and cardiovascular surgery. 2011;142:995-1001

36. Enriquez-Sarano M, Miller FA, Jr., Hayes SN, Bailey KR, Tajik AJ, Seward JB. Effective mitral regurgitant orifice area: Clinical use and pitfalls of the proximal isovelocity surface area method. Journal of the American College of Cardiology. 1995;25:703-709 


\section{Chapter 8}

Left atrial strain and strain rate before and following restrictive annuloplasty for ischemic mitral regurgitation evaluated by two-dimensional speckle tracking echocardiography

Leen Van Garsse, MD, Sandro Gelsomino, MD, PhD, Fabiana Lucà, MD, Orlando Parise, $\mathrm{MS}_{\mathrm{C}}$, EmileCheriex, MD, PhD, Carmelo Massimiliano Rao, MD, Gian Franco Gensini, MD, Jos Maessen, MD, PhD.

Published in: Eur Heart J Cardiovasc Imaging. 2012 Oct 10. In Press. 


\section{Abstract}

Aims: We retrospectively evaluated left atrial (LA) strain and strain Rate (SR) before and after undersized mitral ring annuloplasty (UMRA) for chronic ischemic mitral regurgitation (CIMR) with two-dimensional speckle-tracking echocardiography (2D-STE).

Methods: LA volumes, LA reservoir, conduit, and contractile phases and LA ejection fraction (LAEF) were measured in 95 CIMR patients who underwent coronary bypass grafting (CABG) and UMRA. Left atrial peak global strain $(\varepsilon)$ and reservoir $\left(\mathrm{SR}_{\mathrm{P}}\right)$, conduit $\left(S R_{E}\right)$ and contractile phase $\left(S_{A}\right)$ strain rates were obtained at baseline and at followup (median 41.5 months, interquartile range 23-61). Based on the recurrence of mitral regurgitation (MR) at the follow-up, the patients were divided into 2 groups: patients with (Group MR+, $n=30$ ) or without (Group MR-, $n=65$ ) recurrent MR. Twenty age-and gender-matched healthy adults were controls.

Results: In the MR- Group baseline $\varepsilon(p<0.001), \mathrm{SR}_{\mathrm{P}}(\mathrm{p}<0.001), \mathrm{SR}_{E}(\mathrm{p}<0.001)$ and $\mathrm{SR}_{\mathrm{A}}$ $(p<0.001)$ were enhanced while in $M R+$ Group $\varepsilon(p<0.001), \operatorname{SR}_{P}(p=0.03), \mathrm{SR}_{E}(p=0.03)$ and $\mathrm{SR}_{\mathrm{A}}(p=0.003)$ were worse than controls. At follow up none of these indices changed in the MR+ group while all returned to normal values in patients belonging to the MR- group. LA deformation correlated with corresponding volumetric parameters. Furthermore, we found a direct correlation between $\mathrm{SR}_{\mathrm{E}}$ and early peak diastolic velocity (E) $(\rho=0.52, p=0.02)$ and $E$-wave deceleration time (DT) $(\rho=0.50, p=0.02)$. Finally, there was a strong correlation between $\varepsilon, \operatorname{SR}_{\mathrm{P}}$ and $\mathrm{SR}_{\mathrm{A}}(\rho=0.72, \mathrm{p}<0.001$ and $\rho=0.79$, $p<0.001$, respectively) and $\mathrm{SR}_{E}(\rho=0.69, p<0.001$ and $\rho=0.71, p<0.001$, respectively). Finally, $\varepsilon, \mathrm{SR}_{\mathrm{P}}$ and $\mathrm{SR}_{\mathrm{E}}$ (all, $\mathrm{p}<0.001$ ) were co-factors associated to recurrent $\mathrm{MR}$.

Conclusions: Left atrial peak global strain, peak systolic strain rate and peak early diastolic strain rate were cofactors associated to recurrent MR. The assessment of LA strain and strain rate, in addition to other echocardiographic parameters, can be helpful in detecting patients undergoing UMRA who are unlikely to benefit from annuloplasty. 


\section{Introduction}

Chronic ischemic mitral regurgitation (CIMR) is a functional insufficiency of the mitral valve which commonly complicates myocardial infarction (MI) and severely affects cardiovascular mortality and morbidity ${ }^{1}$. Several studies have provided a great amount of insight into the mechanisms of $\mathrm{CIMR}^{2}$. Increased tethering forces (papillary muscles displacement leading to a more apical position of the leaflets and their coaptation point) and reduced closing forces (reduced contractility, dyssynchrony of the papillary muscles, intra-left ventricular dyssynchrony) appear to be the basic mechanisms for CIMR. Annular dilatation and LV dysfunction probably contribute to the development of $M R$ in the presence of augmented tethering ${ }^{3,4}$. Transthoracic and transesophageal echocardiography has emerged as a fundamental tool in the evaluation of mitral deformation and global and regional left-ventricular remodeling ${ }^{5}$. However, an increasing amount of research has been focused mainly on defining more precisely the annular, sub-annular and ventricular changes occurring in CIMR in order to direct surgical strategy more effectively ${ }^{6,7}$. In contrast, left atrial (LA) function has been poorly investigat$\mathrm{ed}^{8}$ and there is no information about changes in LA function before and after mitral valve repair for CIMR.

Two-dimensional speckle tracking echocardiography (2D-STE) is a non-Doppler based method originally employed to assess left ventricular strain in standard B-mode echocardiography ${ }^{9,10}$. More recently, this technique has been proposed as a method for the quantification of the LA myocardial deformation ${ }^{8}$ and it has been demonstrated to assess accurately atrial function during the different phases of the cardiac cycle ${ }^{11}$.

The aim of this study was to investigate the LA strain and strain rate by 2D-STE before and after mitral valve repair in patients with CIMR. We also tested the ability of LA echocardiographic indices to predict MR recurrence in patients with CIMR undergoing mitral repair.

\section{Materials and methods}

\section{Subjects}

We retrospectively evaluated 249 consecutive patients with CIMR undergoing combined coronary artery bypass grafting (CABG) and mitral valve repair at the University Hospital of Maastricht (Maastricht, The Netherlands) between January 2001 and January 2011.

Inclusion Criteria were ${ }^{7}$ : (1) mild-to-severe MR with prior myocardial infarction >16 days; (2) $75 \%$ or greater stenosis of at least one coronary vessel; (3) a corresponding regional wall motion abnormality; (4) restricted-motion type leaflet dysfunction with or without annular dilatation. 
Exclusion criteria were: (1) persistent/residual MR ( $M R \geq 2+$ at discharge); (2) degenerative or other non-ischemic etiology; (3) acute ischemic mitral regurgitation; (4) additional mitral valve repair procedures; (5) other valvular or congenital heart diseases; (6) severe tricuspid regurgitation; (7) previous cardiac surgery/percutaneous transluminal coronary angioplasty; (8) atrial fibrillation.

Patients were also excluded in case of echocardiograms not available/incomplete or images not appropriate for 2D-STE analysis. Ninety-five patients surviving surgery met all the inclusion criteria and represented our study population. Based on the recurrence of mitral regurgitation (MR) at the follow-up ( $M R \geq 2+$ in patients with no/trivial $M R$ at discharge), the patients were divided into 2 groups: patients with (Group MR+, $\mathrm{n}=30$ ) or without (Group MR-, $\mathrm{n}=65$ ) recurrent MR. Twenty age- and gender-matched healthy adults were controls. Patient characteristics are shown in Table 1. No differences were found in baseline characteristics between the 2 groups. Compared with the MR-Group, the MR+ Group had a higher postoperative New York Heart Association class and showed a trend towards a higher preoperative MR grade.

Clinical follow-up information was obtained from all survivors through outpatient visits and phone calls and was $100 \%$ complete. Median follow up was 41.5 months [interquartile range (IQR)23-61].

Ethical Committee approval was waived due to the retrospective analysis of the study according to the national law regulating observational retrospective studies (Dutch WMO law). However, all patients gave their informed consent to access their data for scientific purposes. 
Table 1. Clinical Characteristics $(n=95)$

\begin{tabular}{|c|c|c|c|}
\hline & $\begin{array}{c}\text { MR+ Group } \\
(n=30)\end{array}$ & $\begin{array}{c}\text { MR- Group } \\
(n=65)\end{array}$ & $\mathrm{p}$ \\
\hline Age, y & $66.3 \pm 4.8$ & $66.5 \pm 7.0$ & 0.4 \\
\hline Gender M/F & $17 / 13(56.6 / 43.4)$ & $39 / 26(60.0 / 40.0)$ & 0.1 \\
\hline NYHA class & $3[3-4]$ & $3[3-4]$ & $>0.9$ \\
\hline CCS angina class & $2[1-3]$ & $2[1-3]$ & $>0.9$ \\
\hline \multicolumn{4}{|l|}{ Euroscore } \\
\hline Additive & 8.8 [7.4-11.9] & $8.7[7.5-12.0]$ & 0.9 \\
\hline Logistic & 14.9 [11.7-16..9] & 14.4 [11.3-16.6] & 0.8 \\
\hline Hypertension & $12(40.0)$ & $27(41.5)$ & 0.8 \\
\hline Diabetes & $7(23.3)$ & $19(29.2)$ & 0.09 \\
\hline COPD & $3(10 . .0)$ & $7(10.7)$ & 0.5 \\
\hline Chronic renal disease & $2(6.7)$ & $5(7.6)$ & 0.7 \\
\hline Cerebral vascular disease & $3(10.0)$ & $7(10.7)$ & 0.8 \\
\hline Peripheral vascular disease & $2(6.7)$ & $5(7.6)$ & 0.7 \\
\hline Familiar history & $13(43.3)$ & $25(38.4)$ & 0.07 \\
\hline IABP & $2(6.7)$ & $6(9.2)$ & 0.1 \\
\hline \multicolumn{4}{|l|}{ Myocardial infarction } \\
\hline Inferior/Posterior & $12(40.0)$ & $27(41.5)$ & \\
\hline Anterior/Septal & $2(6.7)$ & $5(7.6)$ & 0.5 \\
\hline Lateral & $2(6.7)$ & $4(6.3)$ & \\
\hline Combined & $14(46.6)$ & $29(44.6)$ & \\
\hline Coronary vessel disease & $2[2-3]$ & $2[2-3]$ & $>0.9$ \\
\hline Left Main & $7(23.3)$ & $14(21.5)$ & 0.7 \\
\hline \multicolumn{4}{|l|}{ Medications } \\
\hline ACEI/ARB & $28(93.3)$ & $59(90.0)$ & \\
\hline B-adrenergic blockers & $15(50.0)$ & $33(50.7)$ & \\
\hline Long-acting nitrates & $12(40.0)$ & $27(41.5)$ & 0.61 \\
\hline Diuretics & $27(90.0)$ & $55(84.6)$ & \\
\hline Spironolactone & $19(63.3)$ & $38(58.4)$ & \\
\hline \multicolumn{4}{|l|}{ Surgery } \\
\hline CPB time (min) & 115 [92-133] & $108[90-120]$ & 0.1 \\
\hline CCL time (min) & $98[74-111]$ & $88[69-104]$ & 0.08 \\
\hline Mitral Ring size (mm) & $28[26-28]$ & $28[26-28]$ & $>0.9$ \\
\hline Anastomoses/patient & $2[2-3]$ & $2[2-3]$ & $>0.9$ \\
\hline Arterial grafts/patient & $1[1-2]$ & $1[1-2]$ & $>0.9$ \\
\hline \multicolumn{4}{|l|}{ Follow-up } \\
\hline NYHA class & $2[2-3]$ & $1[1-2]$ & $<0.001$ \\
\hline NYHA III/IV & $23(76.6)$ & $20(30.7)$ & $<0.001$ \\
\hline
\end{tabular}

Normally-distributed variables are presented as mean \pm standard deviation. Discrete variables are presented as number (percentage). Non-normally distributed variables are presented as median [Interquartile range]. Abbreviations: $\mathrm{M} / \mathrm{F}=$ Male/Female; NYHA = New York Heart Association; $\mathrm{CCS}=$ Canadian Cardiovascular Society; COPD = Chronic obstructive pulmonary disease; IABP = Intra-aortic balloon pump; $A C E I=$ angiotensin-converting enzyme inhibitor; and $\mathrm{ARB}=$ angiotensin receptor blocker; $\mathrm{CPB}=$ Cardiopulmonary bypass; $\mathrm{CCL}=$ (Aortic) Cross-clamp; $\mathrm{CABG}=$ Coronary artery bypass grafting; $\mathrm{MR}=$ Mitral regurgitation . 


\section{Surgery}

All patients underwent associated CABG. For the purpose of this study, complete revascularization was accomplished when at least one graft was placed distal to an approximately $50 \%$ diameter narrowing in each of the 3 major vascular systems in which arterial narrowing of this severity was noted in a vessel of $1.5 \mathrm{~mm}$ or greater of diameter. It was not considered necessary to bypass all obstructed diagonal branches of the anterior descending or marginal branches of the circumflex coronary arteries for a classification of complete revascularization. Following this definition, $100 \%$ of patients underwent complete revascularization. The ring size was determined by means of standard measurements of the inter-trigonal distance and anterior leaflet height. A downsizing by 2 ring sizes was performed in all patients. After cardiopulmonary bypass, transesophageal echocardiographic analysis was performed to assess residual MR.

\section{Echocardiography}

Transthoracic echocardiography (TTE) was performed within a week before surgery and at follow up appointments. Exams were carried out using a commercially available ultrasound system (IE33,Philips Medical System, Amsterdam, The Netherlands. Images were stored in DICOM format and transferred to a workstation for further off-line analysis (TOMTEC Imaging system, Unterschleißheim, Germany). Measurements and calculations were made separately by one of the investigators (F.L.). The reliability of echocardiographic measurements was assessed by calculating the intra-observer intervals of agreement of the main direct measures used in this study in 20 subjects randomly chosen from among the study patients (Appendix 1).

\section{Mitral regurgitation and $L V$ remodeling}

The following quantitative measurements were simultaneously used to grade the severity of MR: (1) Pulsed Doppler quantitative flow methods and (2) proximal isovelocity surface area (PISA). When the evidence from different parameters was congruent, the measurements were averaged, allowing the calculation of effective regurgitant orifice area (EROA), regurgitant volume (RV) and regurgitant fraction (RF) $)^{12}$.

When different parameters were contradictory, PISA was chosen in case of a central jet or in the presence of a calcific mitral valve or mitral annulus, whereas pulsedwave Doppler was preferred when the jet was eccentric or multiple ${ }^{12}$. Mitral valve configuration was assessed in mid-systole using the parasternal long-axis and fourchamber views. The tenting area (TA), coaptation height $(\mathrm{CH})$, coaptation length $(\mathrm{CL})$ and coaptation distance $(C D)$ were measured as previously reported ${ }^{7}$. 
Left ventricular (LV) volumes and LV ejection fraction were assessed using the bi-apical Simpson disk method and indexed to body surface area (BSA) ${ }^{13}$. Sphericity indexes were obtained at end diastole and end systole $\left(\mathrm{SI}_{\mathrm{D}}\right.$ and $\mathrm{SI}_{\mathrm{S}}$, respectively) as the volume of the LV divided by the volume of a sphere with a diameter equal to the longest axis of the LV measured in the apical view ${ }^{14}$. The wall motion score index (WMSI) was calculated according to a 17 -segment model ${ }^{15}$.

\section{Diastolic function and tissue doppler imaging}

To assess diastolic function, pulsed-wave Doppler of the mitral valve (MV) was performed by placing the Doppler sample volume between the tips of the mitral leaflets. The early (E) and late (A) peak diastolic velocities and E-wave deceleration time (DT) were measured $^{16}$. The isovolumetric relaxation time (IVRT) was obtained by continuous wave Doppler by placing the cursor in the LV outflow tract to simultaneously display the end of aortic ejection and the onset of mitral inflow ${ }^{17}$. Diastolic function was graded according to the most recent recommendations of the European Association of Echocardiography (EAE) /American Society of Echocardiography (ASE) ${ }^{17}$.

\section{LA volumes and LA function}

LA antero-posterior diameter was measured from the parasternal long-axis view at end-systole. LA volumes were measured using the area-length method, from the apical four and two chamber views.

The following LA volumes were measured ${ }^{18}$ :

1. Maximum LA volume ( $\left.\mathrm{LAV}_{\mathrm{MAX}}\right)$ : just prior to $\mathrm{MV}$ opening

2. $L A$ volume prior to atrial contraction $\left(\operatorname{LAV}_{\mathrm{P}}\right)$ : just prior to $E C G \mathrm{p}$ wave

3. Minimum LA volume ( $\left(\mathrm{AV}_{\mathrm{MIN}}\right)$ : immediately after $\mathrm{MV}$ closure.

From these volumes, the following dynamic volumes were calculated:

a. $L A$ reservoir volume $\left(L A V_{\text {RES }}\right): L A V_{M A X}-L A V_{\text {MIN }}$

b. $L A$ passive emptying volume $\left(L A V_{P-E M P T}\right): L A V_{M A X}-L A V_{P}$

c. $L A$ contractile volume $\left(\operatorname{LAV}_{\text {CONTR }}\right)$ : $L_{A V}-L_{P} V_{M I N}$

LA volumes were indexed to BSA as recommended ${ }^{19}$.

LA ejection fraction (LAEF) was calculated as LA contractile volume/LAV $\mathrm{V}_{\mathrm{p}}$, and expressed as a percentage.

\section{Two-dimensional speckle-tracking echocardiography}

Longitudinal LA strain was computed by speckle-tracking echocardiography (2D Cardiac Performance Analysis; Tom Tec Imaging Systems, Munich, Germany). Twelve global 
strain curves were obtained using apical four-chamber and two-chamber views: basal septal, mid septal, apical septal, basal lateral, mid lateral, apical lateral, basal anterior, mid anterior, apical anterior, basal inferior, mid inferior and apical inferior. Global LA peak systolic strain $(\varepsilon)$ was measured during LV ejection (Figure $1 \mathrm{~A})$. Peak strain rate $\left(S R_{P}\right)$ was measured during LV ejection (LA reservoir phase) whereas peak early diastolic strain rate $\left(S R_{E}\right)$ was measured during LV early diastole (LA conduit phase) and peak negative strain rate $\left(S_{A}\right)$ was measured during $L V$ diastole occurring after the P-wave (active contraction phase, Figure $1 \mathrm{~B}$ ). Deformation parameters were also normalized to relative volumes to correct for the volume-dependency of the deformation.
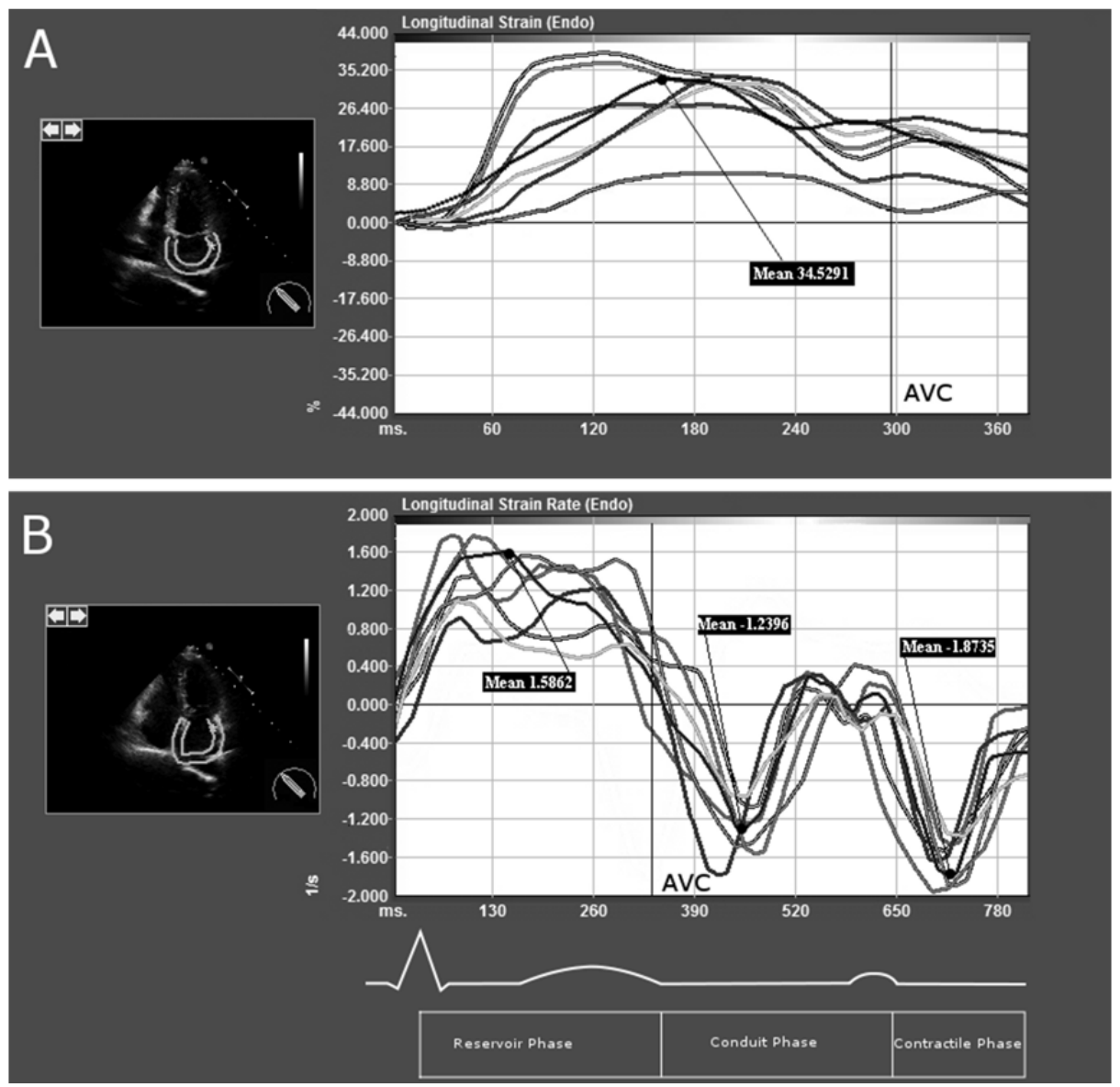

Figure 1. A) Left atrial peak systolic strain and B) strain rate with schematic ECG in a control subject. Abbreviations. AVC: Aortic Valve Closure. 


\section{Statistical analysis}

Data were analyzed with the use of statistical software (SPSS for Windows, version 12.0; SPSS Inc., Chicago, III). Normality of continuous data was analyzed with the Kolmogorov-Smirnov test. Variables were expressed as mean \pm SD, median and interquartile range, or number of patients (percentage) as appropriate. For within-group comparisons before and after annuloplasty, the paired t test or the Wilcoxon signed rank test were used. Between-group differences were assessed by the unpaired test, Mann-Whitney $U$ test, or Pearson $\chi^{2}$ test. Spearman Rank Correlation was employed to test the correlation between variables. The Benjamini and Hochberg step-up false discovery rate $(F D R)^{20}$ control correction for multiple testing was applied to the $p$ values in the analysis $\left(\mathrm{p}_{\mathrm{FDR}}\right)$.

Univariable regression was carried out with postoperative regurgitant volume as dependent variable and preoperative LA echocardiographic parameters (Left Atrial Diameter [LAD], LAV $\mathrm{MAX}_{1}, \mathrm{LAV}_{\mathrm{P}}, \mathrm{LAV}_{\mathrm{MIN}}, \mathrm{LAV}_{\mathrm{RES}}, \mathrm{LAV}_{\mathrm{P}-\mathrm{EMPT}}, \mathrm{LAV}_{\text {CONTR, }} \mathrm{LAEF}, \varepsilon, \mathrm{SR}_{\mathrm{P}}, \mathrm{SR}_{\mathrm{E}}$ and $\mathrm{SR}_{\mathrm{A}}$ ) as independent variables. Variables reaching statistical significance or borderline $(p \leq 0.1)$ were introduced in multivariable analysis. Multivariable logistic regression with the forward stepwise method was performed to identify co-factors associated to significant post-repair MR. The goodness of fit of the final logistic regression models was assessed with the Hosmer-Lemeshow (H-M) statistic and predictive accuracy was assessed by the concordance index (c). Optimal cut-off values were determined by receiver operating characteristic curve (ROC) as the rounding cut-off that gives the maximum sum of sensitivity and specificity. This value should be the shoulders at the top left of the ROC curve. Results were validated using the bootstrap method (1000 iterations).

To test whether the predictive value of LA parameters were independent of diastolic function a sub-group analysis was carried out based on deceleration time (DT, $\leq 142$ / >142 ms) which was found to be a strong predictor of recurrent MR in our previous study ${ }^{21}$. We tested for interactions by entering interaction terms between each LA parameter and DT with an interaction $p<0.10$ considered statistically significant.

\section{Results}

\section{Mitral regurgitation and $L V$ remodeling}

Patients in the MR+ Group had baseline larger LV volumes (both, $p<0.001$ ) and a more spherical ventricle ( $p<0.001$, Table 2). At follow up, in patients in the MR- Group, ESVI decreased significantly $(p<0.001)$ while in group MR+ Group it increased compared to baseline value without reaching statistical significance $(p=0.7)$. Changes in EDVI and WMSI showed the same tendency for ESVI. Similarly, end-diastolic diameter $(p<0.001)$ 
and end-systolic diameter $(p=0.004)$ decreased in MR-patients, whereas did not change in MR+ Group ( $p=0.8$ and $p=0.7$, respectively).

Systolic and diastolic sphericity indexes were reduced significantly in the MRGroup ( $p=0.003$ and $p<0.001$, respectively), whereas these indexes increased, though not significantly, in patients with recurrent $M R$ (both, $p=0.4$ ). At baseline there was no significant difference regarding quantitative $M R$ data. In contrast, preoperative TA $(p=0.02)$ and $\mathrm{CH}(p=0.01)$ were higher whereas $\mathrm{CL}$ was lower $(p=0.002)$ in patients with recurrent $M R$. In contrast, $C D$ was comparable between groups $(p=0.6)$. At follow up, in the MR- Group, TA $(p<0.001)$ and $\mathrm{CH}(p=0.01)$ were reduced while $C L(<0.001)$ increased significantly. These indices did not change in the MR+ Group.

Table 2. LV Geometry and Mitral Valve Regurgitation ( $n=95)$.

\begin{tabular}{|c|c|c|c|c|c|c|c|}
\hline & \multirow[b]{2}{*}{ Controls } & \multicolumn{3}{|c|}{$\begin{array}{c}\text { MR+ Group } \\
(n=30)\end{array}$} & \multicolumn{3}{|c|}{$\begin{array}{c}\text { MR- Group } \\
(n=65)\end{array}$} \\
\hline & & Preoperative & Follow up & $\mathrm{p}$ & Preoperative & Follow up & $p$ \\
\hline$\overline{\text { LVEF (\%) }}$ & $59 \pm 7$ & $38 \pm 10$ & $37 \pm 9$ & 0.8 & $39 \pm 10^{*}$ & $42 \pm 10$ & 0.07 \\
\hline $\mathrm{EDD}(\mathrm{mm})$ & $44 \pm 5$ & $59 \pm 8^{*}$ & $60 \pm 9^{* \ddagger}$ & 0.8 & $60 \pm 8^{*}$ & $47 \pm 7$ & $<0.001$ \\
\hline $\mathrm{ESD}(\mathrm{mm})$ & $30 \pm 4$ & $45 \pm 6^{*}$ & $47 \pm 8^{* \ddagger}$ & 0.7 & $44 \pm 6^{*}$ & $35 \pm 5$ & 0.004 \\
\hline $\operatorname{EDVI}\left(\mathrm{mL} / \mathrm{m}^{2}\right)$ & $50 \pm 10$ & $129 \pm 24^{*+}$ & $134 \pm 22^{* \ddagger}$ & 0.1 & $105 \pm 15^{*}$ & $80 \pm 12$ & $<0.001$ \\
\hline $\operatorname{ESVI}\left(\mathrm{mL} / \mathrm{m}^{2}\right)$ & $22 \pm 5$ & $81 \pm 14^{*+}$ & $83 \pm 15^{* \ddagger}$ & 0.7 & $63 \pm 10^{*}$ & $47 \pm 8$ & $<0.001$ \\
\hline WMSI & $1.0 \pm 0$ & $1.5 \pm 0.4^{* \dagger}$ & $1.6 \pm 0.4^{*+}$ & 0.8 & $1.3 \pm 0.2^{*}$ & $1.1 \pm 0.2$ & 0.03 \\
\hline$S I_{D}$ & $0.42 \pm 0.1$ & $0.80 \pm 0.1^{*+}$ & $0.85 \pm 0.1^{* \ddagger}$ & 0.4 & $0.72 \pm 0.1^{*}$ & $0.62 \pm 0.1$ & 0.003 \\
\hline $\mathrm{SI}_{\mathrm{S}}$ & $0.35 \pm 0.1$ & $0.75 \pm 0.1^{* \dagger}$ & $0.80 \pm 0.1^{* \ddagger}$ & 0.4 & $0.64 \pm 0.1^{*}$ & $0.51 \pm 0.1$ & $<0.001$ \\
\hline$\overline{M R}$ & - & $3[3-4]$ & $3[3-4]$ & $>0.9$ & $3[3-4]$ & $1[1-2]$ & $<0.001$ \\
\hline $\operatorname{ERO}\left(\mathrm{mm}^{2}\right)$ & - & $34.6 \pm 12.2$ & $29.4 \pm 10.1^{\ddagger}$ & 0.07 & $36.6 \pm 10.9$ & -1 & - \\
\hline $\mathrm{RF}(\%)$ & - & $48.3 \pm 12.1$ & $43.9 \pm 12.6^{\ddagger}$ & 0.07 & $45.9 \pm 10.3$ & - & - \\
\hline $\mathrm{RV}$ (ml/beat) & - & $59.0 \pm 10.3$ & $55.8 \pm 13.9^{\ddagger}$ & 0.3 & $57.7 \pm 11.9$ & - & - \\
\hline $\mathrm{TA}\left(\mathrm{cm}^{2}\right)$ & $0.8 \pm 0.2$ & $4.0 \pm 1.1^{*+}$ & $3.6 \pm 1.4^{\ddagger}$ & 0.07 & $3.1 \pm 1.2^{*}$ & $1.9 \pm 0.2$ & $<0.001$ \\
\hline $\mathrm{CL}(\mathrm{mm})$ & $8.5 \pm 0.4$ & $3.2 \pm 0.3^{*^{+}}$ & $3.3 \pm 0.3^{\ddagger}$ & 0.8 & $4.2 \pm 0.5^{*}$ & $8.7 \pm 0.5$ & $<0.001$ \\
\hline $\mathrm{CD}(\mathrm{mm})$ & $25.5 \pm 5.9$ & $38.6 \pm 5.4^{*}$ & $38.1 \pm 5.8^{\ddagger}$ & 0.8 & $35.5 \pm 6.6^{*}$ & $31.0 \pm 4 . .3$ & 0.44 \\
\hline $\mathrm{CH}(\mathrm{mm})$ & $6.0 \pm 1.9$ & $13.0 \pm 2.0^{*+}$ & $12.6 \pm 1.7^{\ddagger}$ & 0.6 & $10.0 \pm 2.1^{*}$ & $6.5 \pm 0.6$ & 0.001 \\
\hline
\end{tabular}

Normally- distributed variables are presented as mean \pm standard deviation. Non-normally distributed variables are presented as median [Interquartile range].

Abbreviations: $\mathrm{LVEF}=$ Left Ventricular Ejection Fraction; EDD = End-diastolic diameter; ESD = End-systolic diameter; $E S V I=$ End-systolic Volume Index; EDVI = End-diastolic Volume Index; WMSI = Wall motion score index; $\mathrm{SI}_{\mathrm{D}}=$ diastolic sphericity Index; $\mathrm{SI}_{\mathrm{S}}=$ Systolic sphericity Index; $\mathrm{MR}=$ Mitral Regurgitation; $\mathrm{ERO}=\mathrm{Effec}-$ tive Regurgitant Orifice; RF = Regurgitant Fraction; RV = Regurgitant Volume; $\mathrm{TA}=$ Tenting Area; $\mathrm{CL}=\mathrm{Coapta}$ tion Length; $\mathrm{CD}=$ Coaptation Distance; $\mathrm{CH}=$ Coaptation Height. ${ }^{*} \mathrm{p}<0.05$ vs. controls; ${ }^{\dagger} \mathrm{p}<0.05$ vs. MR- at baseline; ${ }^{\ddagger} p<0.05$ vs. MR- at follow-up.

\section{Diastolic function}

In the MR+ Group diastolic dysfunction was mild (E/A $<0.8$, DT $>200 \mathrm{~ms}$, IVRT $\geq 100$ $\mathrm{ms}$ ) in 6 patients $(20 \%)$, moderate (E/A $<0.8-1.5$ [decreasing by $50 \%$ during Valsalva maneuvers], DT $160-200 \mathrm{~ms}$, IVRT 60-100 ms) in 16 patients $(53.4 \%)$ and severe (E/A $\geq$ 2 , DT $<160 \mathrm{~ms}$, IVRT $\leq 60 \mathrm{~ms}$ ) in 8 patients (26.6\%). In the MR-group these figures 
were 38 (58.5\%), 15 (23.1\%) and 5 (7.7\%, p=0.002). In addition, in this Group, 7 (10.7\%) patients had no diastolic dysfunction. In the MR+ Group, at baseline, $E / A$ ( $p=0.04$ vs. controls, $p<0.001$ vs. MR-), DT ( $<<0.001$ vs. controls, $p<0.001$ vs. MR-) and IVRT ( $p=$ 0.03 vs. controls, $p=0.02$ vs. MR-) were lower compared to controls and MR-patients. None of these indices varied in the MR+ Group at follow-up. In contrast, in patients without recurrent $M R, E / A(p<0.001)$ was reduced while the other indices did not change (Figure $2 \mathrm{~A}-\mathrm{C}$ ).

A

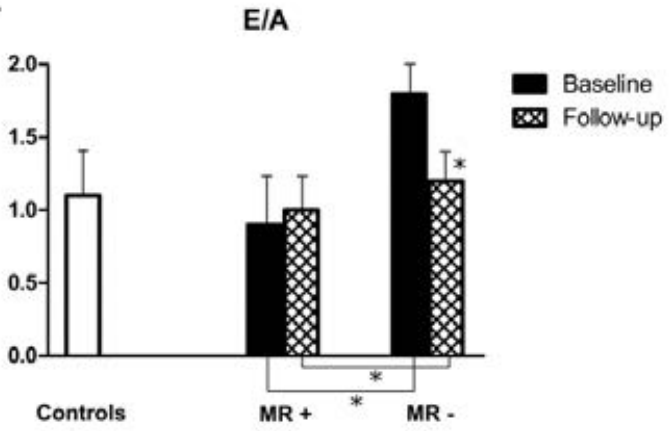

B

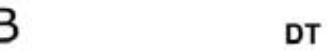

Figure 2. Diastolic function in controls and at baseline and follow up in patients with $(M R+)$ or without (MR-) recurrent Mitral Regurgitation. A) Early (E) and late (A) peak diastolic velocities ratio E/A. B) E-wave deceleration time (DT). C) Isovolumetric relaxation time (IVRT). ${ }^{*} \mathrm{p}<0.05$.

C

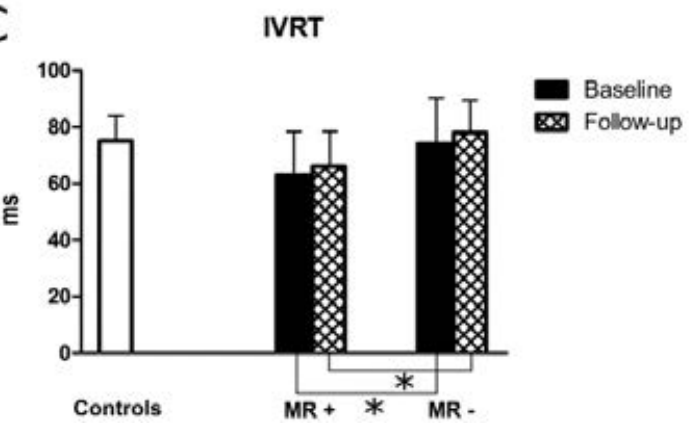




\section{LA function and 2D-STE analysis}

An higher number of patients in MR+ Group experienced at least one episode of $\mathrm{AF}$ after surgery (52.9\% [18/34] vs. 5.0\% [6/118], p<0.001).

Compared to controls, all static and dynamic atrial volumes were significantly higher in MR- and significantly lower in MR+ patients (Table 3). At follow up, in patients without recurrent MR all volumes were reduced and were not significantly different from controls. In contrast, for $\mathrm{MR}+$ patients atrial volumes did not vary. Baseline $\mathrm{LA}_{\mathrm{EF}}$ was comparable between Groups and not different from controls. At follow up it did not change in both Groups. In the MR+ Group $\varepsilon(p<0.001$, normalized $p=0.006), S R_{P}$ $(p=0.03$, normalized $p=0.04), \mathrm{SR}_{E}(p=0.03$, normalized $p=0.04)$ and $\mathrm{SR}_{A}(p=0.003$, normalized $p=0.01$ ) were lower (Figure $3 A$, Figure $4 A$ ) while in $M R$ - patients $\varepsilon(p<0.001$, normalized $p<0.001), S_{P}(p<0.001$, normalized $p<0.001), \operatorname{SR}_{E}(p<0.001$, normalized $p<0.001)$ and $S_{A}(p<0.001$, normalized $p<0.001)$ were higher (Figure $3 C$, Figure $4 C$ ) than controls (Figure $1 \mathrm{~A}$, Figure $1 \mathrm{~B}$ ). At follow up none of these indices changed in the $\mathrm{MR}+$ Group (Figure 3B, Figure $4 \mathrm{~B}$ ) while all returned to normal values in patients belonging to the MR- Group (Figure 3D, Figure 4D).

Table 3. LA Function and Speckle-tracking Analysis

\begin{tabular}{|c|c|c|c|c|c|c|c|}
\hline & \multirow[b]{2}{*}{ Controls } & \multicolumn{3}{|c|}{$\begin{array}{c}M R+\text { Group } \\
(n=34)\end{array}$} & \multicolumn{3}{|c|}{$\begin{array}{c}\text { MR- Group } \\
(n=118)\end{array}$} \\
\hline & & Preoperative & Follow up & $\mathrm{p}$ & Preoperative & Follow up & $P$ \\
\hline $\mathrm{LAD}\left(\mathrm{cm} / \mathrm{m}^{2}\right)$ & $1.8 \pm 0.2$ & $2.2 \pm 0.3^{*+}$ & $2.2 \pm 0.3^{* \ddagger}$ & 0.9 & $2.4 \pm 0.3^{*}$ & $2.0 \pm 0.3^{*}$ & 0.01 \\
\hline $\mathrm{LAV}_{\mathrm{MAX}}\left(\mathrm{mL} / \mathrm{m}^{2}\right)$ & $39 \pm 15$ & $28 \pm 13^{*+}$ & $30 \pm 12^{* \ddagger}$ & 0.8 & $62 \pm 18^{*}$ & $42 \pm 10$ & $<0.001$ \\
\hline $\operatorname{LAV}_{\mathrm{P}}\left(\mathrm{mL} / \mathrm{m}^{2}\right)$ & $28 \pm 8$ & $20 \pm 6^{*+}$ & $20 \pm 7^{* \ddagger}$ & $>0.9$ & $48 \pm 13^{*}$ & $30 \pm 8$ & $<0.001$ \\
\hline $\operatorname{LAV}_{\mathrm{MIN}}\left(\mathrm{mL} / \mathrm{m}^{2}\right)$ & $19 \pm 6$ & $15 \pm 5^{*+}$ & $16 \pm 6^{* \ddagger}$ & 0.8 & $30 \pm 11^{*}$ & $22 \pm 6$ & $<0.001$ \\
\hline $\mathrm{LAV}_{\mathrm{RES}}\left(\mathrm{mL} / \mathrm{m}^{2}\right)$ & $20 \pm 6$ & $12 \pm 5^{*+}$ & $14 \pm 7^{* \ddagger}$ & 0.5 & $34 \pm 10^{*}$ & $21 \pm 6$ & $<0.001$ \\
\hline $\operatorname{LAV}_{\text {P-EMPT }}\left(\mathrm{mL} / \mathrm{m}^{2}\right)$ & $11 \pm 4$ & $8 \pm 2^{*+}$ & $9 \pm 5^{* \neq}$ & 0.7 & $15 \pm 7^{*}$ & $12 \pm 5$ & 0.03 \\
\hline $\operatorname{LAV}_{\text {CONTR }}\left(\mathrm{mL} / \mathrm{m}^{2}\right)$ & $10 \pm 3$ & $7 \pm 2^{*+}$ & $6 \pm 2^{* \neq}$ & $>0.9$ & $18 \pm 8^{*}$ & $11 \pm 4$ & 0.007 \\
\hline LAEF (\%) & $35 \pm 7$ & $33 \pm 6$ & $33 \pm 5$ & 0.8 & $37 \pm 5$ & $36 \pm 6$ & 0.8 \\
\hline$\varepsilon(\%)$ & $35.2 \pm 8.1$ & $22.2 \pm 12.1^{\dagger}$ & $20.6 \pm 12.9^{* \ddagger}$ & 0.6 & $47.2 \pm 11.2^{*}$ & $37.3 \pm 12.4$ & $<0.001$ \\
\hline $\mathrm{SR}_{\mathrm{P}}\left(\sec ^{-1}\right)$ & $1.56 \pm 0.9$ & $1.43 \pm 0.4^{* \dagger}$ & $1.33 \pm 0.3^{* \ddagger}$ & 0.5 & $2.55 \pm 0.8^{*}$ & $1.59 \pm 0.7$ & $<0.001$ \\
\hline $\mathrm{SR}_{\mathrm{E}}\left(\sec ^{-1}\right)$ & $-1.27 \pm 0.5$ & $-1.04 \pm 0.2^{*+}$ & $-1.18 \pm 0.2^{* \ddagger}$ & 0.5 & $-2.32 \pm 0.7^{*}$ & $-1.28 \pm 0.5$ & $<0.001$ \\
\hline $\mathrm{SR}_{\mathrm{A}}\left(\sec ^{-1}\right)$ & $-1.87 \pm 0.6$ & $-1.39 \pm 0.3^{*+}$ & $-1.30 \pm 0.2^{* \ddagger}$ & 0.1 & $-2.64 \pm 0.7^{*}$ & $-1.82 \pm 0.7$ & $<0.001$ \\
\hline
\end{tabular}

Abbreviations: $L A D=$ Left Atrial diameter; $L_{A V} V_{M A X}=$ Maximum Left atrial volume; $L A V_{P}=$ Left atrial volume prior to atrial contraction; $L A V_{M I N}=$ Minimum Left atrial volume; $L A V_{R E S}=$ Reservoir Left atrial volume; $L A V_{P}$ ${ }_{\mathrm{EMPT}}=$ Passive-empting Left atrial volume; $\mathrm{LAV}_{\text {CONTR }}=$ Contraction Left atrial volume; $\mathrm{LAEF}(\%)=$ Left atrial ejection fraction; $\varepsilon=$ Peak systolic atrial strain; $\mathrm{SR}_{\mathrm{P}}=$ Peak systolic strain rate; $\mathrm{SR}_{\mathrm{E}}=$ Peak early diastolic strain rate; $\mathrm{SR}_{\mathrm{A}}=$ Peak late diastolic strain rate. ${ }^{*} \mathrm{p}<0.05$ vs. controls; ${ }^{\dagger} \mathrm{p}<0.05$ vs. $\mathrm{MR}$ - at baseline; ${ }^{\ddagger} \mathrm{p}<0.05$ vs. $\mathrm{MR}-$ at follow-up. 

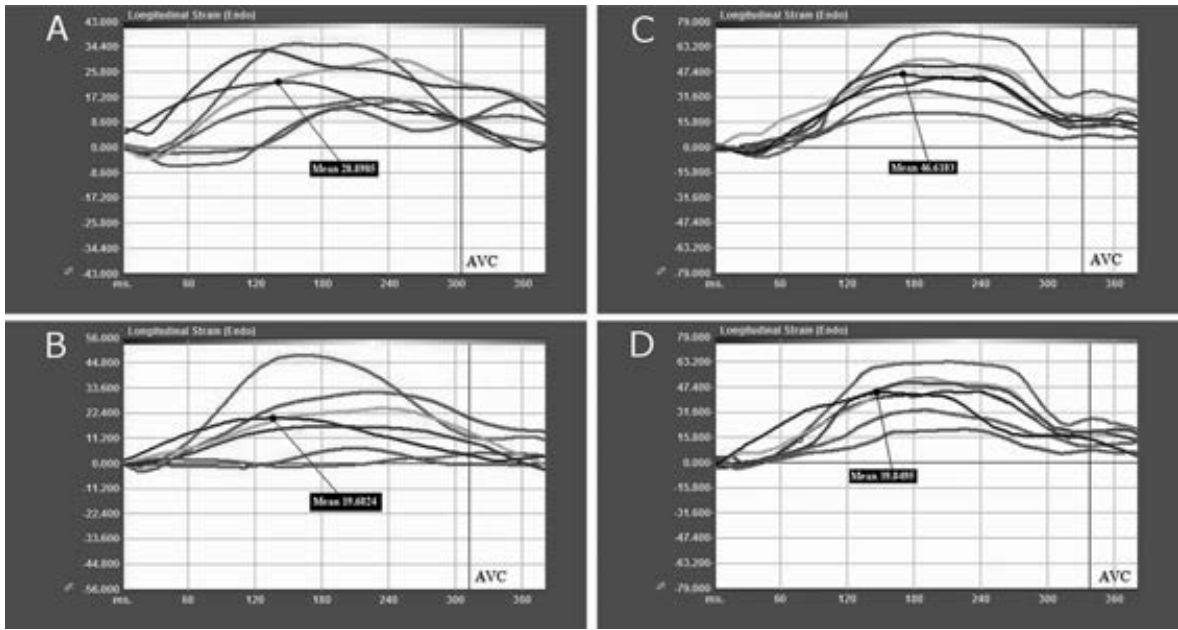

Figure 3. A) Preoperative left atrial peak global strain in a patient with recurrent MR. B) Postoperative left atrial peak global strain in the same patient as in A. C) Preoperative left atrial peak global strain in a patient without recurrent MR. D) Postoperative left atrial peak global strain in the same patient as in C.

Abbreviations. AVC: Aortic valve closure.
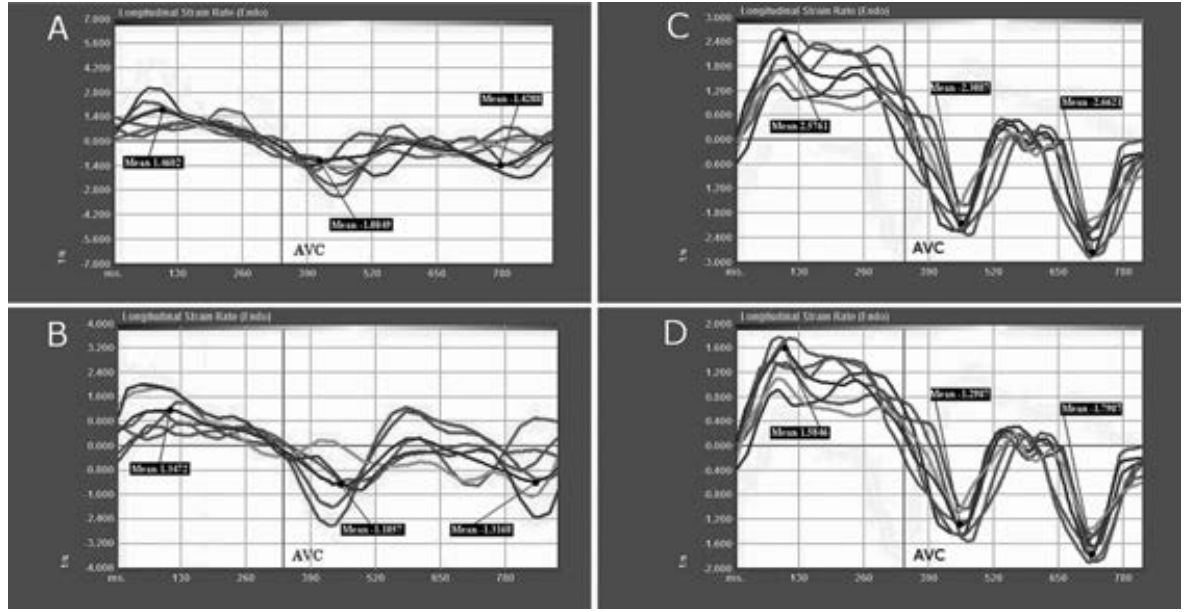

Figure 4. A) Preoperative left atrial Strain Rate in a patient with recurrent MR. B) Postoperative left atrial Strain Rate in the same patient as in A. C) Preoperative left atrial Strain Rate a patient without recurrent MR. D) Postoperative left atrial Strain Rate in the same patient as in C.

Abbreviations. AVC: Aortic valve closure.

\section{Correlations between 2D-STE indices and other echocardiographic parameters}

There was a strong correlation between and $\operatorname{LAV}_{\mathrm{RES}}$ and $\varepsilon\left(\rho=0.64, p=0.001, \mathrm{p}_{\mathrm{FDR}}=0.002\right)$ and $\operatorname{SR}_{\mathrm{P}}\left(\rho=0.57, \mathrm{p}=0.002, \mathrm{p}_{\mathrm{FDR}}=0.003\right)$. Furthermore we found a significant correlation between $\operatorname{SR}_{E}$ and $\operatorname{LAV}_{\mathrm{P} \text {-EMPT }}\left(\rho=0.45, p=0.03, \mathrm{p}_{\mathrm{FDR}}=0.033\right)$ and between $\mathrm{SR}_{\mathrm{A}}$ and $\mathrm{LAV}_{\text {CONTR }}$ 
$\left(\rho=0.40, p=0.03, p_{F D R}=0.033\right)$. Also, we found a direct correlation between $S_{E}$ and $E$ $\left(\rho=0.52, p=0.02, p_{F D R}=0.029\right)$ and DT $\left(\rho=0.50, p=0.02, p_{F D R}=0.029\right)$. In addition, $S R_{A}$ showed a weak correlation with $\operatorname{LAEF}\left(\rho=0.25, p=0.3, p_{F D R}=0.027\right)$. Finally, there was a strong correlation between both $\varepsilon$ and $\mathrm{SR}_{\mathrm{P}}$ with $\mathrm{SR}_{\mathrm{A}}\left(\rho=0.72, p<0.001\left[\mathrm{p}_{\mathrm{FDR}}=0.001\right]\right.$ and $\rho=0.79, p<0.001\left[p_{F D R}=0.001\right]$, respectively) and $S_{E}\left(\rho=0.69, p<0.001\left[p_{F D R}=0.001\right]\right.$ and $\rho=0.71, p<0.001\left[p_{F D R}=0.001\right]$, respectively).

\section{Co-factors of recurrent mitral regurgitation}

Recurrent MR was positively correlated with $\varepsilon(p<0.001), \mathrm{SR}_{\mathrm{P}}(\mathrm{p}<0.001)$, and $\mathrm{SR}_{\mathrm{E}}$ $(p<0.001)$. There was no significant correlation between MR recurrence and other $L A$ echocardiographic parameters.

Logistic regression analysis revealed that $\varepsilon, \mathrm{SR}_{\mathrm{P}}$ and $\mathrm{SR}_{E}$ (all, $\mathrm{p}<0.001$ ) were cofactors associated to recurrent MR. The model proved to be reliable and accurate (Table 4).

At Receiver operating characteristic (ROC) analysis an $\varepsilon$ value $\leq 25 \%$ predicted recurrence of MR with $92 \%$ sensitivity and $87 \%$ specificity (Area Under Curve [AUC] 0.90 [95\% Cl: 0.8-0.98], $\mathrm{p}<0.001$ ); $\mathrm{SR}_{\mathrm{p}}$ had $90 \%$ sensitivity and $82 \%$ specificity with an optimal cut-off of $\leq 1.50 \mathrm{sec}^{-1}$ (AUC 0.85 [95\% Cl: 0.70-0.94], $\mathrm{p}<0.001$ ). SRE with a cut-off $\geq$ $-1.11 \mathrm{sec}^{-1}$ had $86 \%$ sensitivity and $80 \%$ specificity (AUC 0.79 [95\% Cl: 0.66-0.83], p $<0.001)$.

Finally, There were no apparent significant interactions between DT and $\varepsilon$ (interaction $p$ value $=0.21$ ), $S_{P}$ (interaction $p$ value $=0.56$ ) and $S_{E}$ (interaction $p$ value = $0.68)$.

Table 4. Logistic Regression

\begin{tabular}{lccc}
\hline & $\mathrm{p}$ & $\mathrm{OR}$ & $95 \% \mathrm{Cl}^{*}$ \\
\hline$\varepsilon$ & $<0.001$ & 4.8 & $3.0-5.7$ \\
$\mathrm{SR}_{\mathrm{P}}$ & $<0.001$ & 4.4 & $2.6-5.2$ \\
$\mathrm{SR}_{\mathrm{E}}$ & $<0.001$ & 4.0 & $2.3-5.0$ \\
\hline
\end{tabular}

Abbreviations: $\varepsilon=$ Peak systolic strain; $\mathrm{SR}_{\mathrm{P}}=$ Peak systolic strain rate; $\mathrm{SR}_{\mathrm{E}}=$ Peak early diastolic strain rate; $\mathrm{OR}=$ Odds Ratio; $\mathrm{Cl}=$ Confidence Interval. *Bootstrap corrected

\section{Discussion}

The main findings of our study can be summarized as follows:

First, the left atrial peak global strain $\varepsilon$ and $S_{P}$, which accurately reflect the LA reservoir function ${ }^{22-24}$, were increased at baseline in MR-patients, possibly due to a rise in atrial compliance and increased filling during the reservoir period (combined 
flow from pulmonary veins and from the mitral regurgitant jet) ${ }^{25}$. After surgery, in these patients, the reduced preload after MV repair was responsible for the normalization of $\varepsilon$ and $\mathrm{SR}_{\mathrm{p}}$. This was not observed in patients showing recurrent MR who showed an impairment of left atrial peak global strain $(\varepsilon)$ and $\mathrm{SR}_{\mathrm{p}}$ at baseline. At follow up, MR+ patients still had low left atrial peak global strain and peak strain rate and this might be explained by irreversible ultra-structural changes of the atrial wall occurring in these patients ${ }^{26}$.

Second, compared to controls, baseline passive LV filling was increased in patients belonging to the MR- Group. This might be attributable to an increased atrioventricular gradient, decreased LV chamber stiffness and increased recoil of the $L^{27}$. After repair, $\mathrm{SR}_{\mathrm{E}}$ was comparable with controls. In $\mathrm{MR}+$ patients baseline $\mathrm{SR}_{\mathrm{E}}$ was low and this might be explained by the higher levels of LV filling. Indeed, it has been demonstrated that the enhancement of conduit function may diminish with LV dysfunction ${ }^{28}$ which, in this Group, was also accompanied by a decreased E wave DT, consistent with the onset of increased LV stiffness ${ }^{29}$. This was also confirmed by the direct correlation between $\mathrm{SR}_{\mathrm{E}}$ and DT $(\mathrm{p}=0.02)$.

Third, regarding the contraction phase, in the preoperative period patients in the MR- Group showed a higher SR . Importantly, in this Group, preoperative LAEF, which measures booster function ${ }^{30}$, was comparable to controls and this index showed a weak correlation with $\mathrm{SR}_{\mathrm{A}}(\mathrm{p}=0.3)$.

Fourth, the strong correlation between $\mathrm{SR}_{A}$ and both $\varepsilon(p<0.001)$ and $\mathrm{SR}_{\mathrm{P}}(\mathrm{p}<0.001)$ demonstrates the preload-dependency of the contractile phase $\mathrm{SR}_{\mathrm{A}}{ }^{23}$. Indeed, after repair which reduces the preload, $\mathrm{SR}_{\mathrm{A}}$ returned to normal values in $\mathrm{MR}$ - patients. Conversely, the strong reduction of $\mathrm{SR}_{\mathrm{A}}$ in patients with recurrent $\mathrm{MR}$ might be potentially attributable to the high filling pressure and, consequently, to the increased LA afterload resulting in an overwhelmed Frank-Starling mechanism ${ }^{23,}{ }^{31}$. In the postoperative period $\mathrm{SR}_{\mathrm{A}}$ did not improve in $\mathrm{MR}+$ patients, reflecting a persistent state of LV impairment.

Fifth, interestingly, in our experience, although the degree of MR was not different in the two groups, confirming our previous experience ${ }^{32}$, LA volumes were higher in the MR- group, whereas in MR+ groups were markedly lower. Although the exact mechanism remains to be elucidated, we might postulate that this discrepancy may be due to significant LA wall stiffness and impairment in LA compliance (reservoir function) estimated by global peak systolic strain $\varepsilon$ and peak systolic strain rate $\mathrm{SR}_{\mathrm{p}}$ in $\mathrm{MR}+$ patients. However, whether it reflects irreversible histological changes in the left atrium and LA fibrosis will be the subject of ongoing research. After surgery, in accordance to Bax et $\mathrm{al}^{33}$ and Geidel et $\mathrm{al}^{34}$, we observed a significant reduction in static and dynamic volumes only in MR- patients, whereas in the MR+ group they did not vary after surgery. This might further confirm irreversible chronic changes and ultra-structural LA wall abnormalities in MR+ patients. 
Finally, in our experience, $\varepsilon, S R_{P}$ and $S R_{E}($ all, $p<0.001$ ) were multivariable cofactors associated to recurrent MR. In contrast, LA volumes resulted not to be significant. All these parameters resulted to be independent of DT which has been previously recognized a predictor of recurrent $\mathrm{MR}^{21}$.

\section{Study limitations}

Our study findings should be viewed in light of some inherent limitations.

First, data about survival and adverse cardiac events (MACE) were not provided since this was not the aim of the study. Hence, we cannot exclude that the observed strain differences and recurrence of MR might have clinical effect on survival as demonstrated by previous studies ${ }^{35}$. Furthermore, standard echocardiographic results shown in our previous paper were not presented in this study.

Second, although 2D-STE has the advantage of being angle independent and to be affected less by reverberations, side lobes and drop-out artifacts, it carries some intrinsic limitations such as strict frame rate dependency, and potential errors in epicardial/endocardial border tracing in subjects with suboptimal image quality. Although strain measurements in our study were carried out by an experienced echocardiographer, specifically trained for this analysis, these limitations must be considered while examining our findings.

Third, we employed conventional indices of LV diastolic function. Tissue Doppler imaging (TDI) would have been more useful in estimating LV diastolic function. Nonetheless, DT, although not load independent, is strongly related to pulmonary capillary wedge pressure and end-diastolic pressure and it is a useful parameter for the serial assessment of LV diastolic function in post-infarct patients, including those with MR. Moreover, of 249 patients only 95 were included after the exclusion/inclusion criteria. Therefore, the population studied appears rather selective and may not represent the majority of real-life patients. This aspect must be taken into account when examining our results.

Fourth, we excluded patients with atrial fibrillation from this study. It may have been interesting to see how AF would have affected LA myocardial deformation and its correlation with LA structural changes. In addition, we have not assessed pulmonary venous inflow which could have provided more information on LA function. Moreover, it has been demonstrated that age influences LA deformation ${ }^{17}$ but our analysis did not take into consideration the influence of age on LA strain and SR.

Fifth, we did not explore the influence of postoperative AF on MR and strain. We cannot exclude that these patients would probably have more MR, worse LVEF and more severe diastolic dysfunction at baseline, perhaps also more severe coronary heart disease or scarring after myocardial infarction. 
Sixth, only patients surviving surgery were included and there was a large variability in follow-up time. Indeed, after 10 years there might be more MR recurrence as LVEF deteriorates in CABG patients, as compared to shorter follow-up.

Seventh, the timing after intervention for the control examination has a wide range and this should normally affect the relationship between recurrent MR and LA function. This aspect must be taken into consideration when examining our findings.

Finally, since LV volumes are higher in the MR+ Group, we cannot exclude that the recurrence of MR may be just related to more advanced underlying myocardial disease and its progression. Hence, the LA strains might therefore just identify the more advanced disease and may not have any direct causal association with recurrence of MR.

Nonetheless, even with the above mentioned limitations, to the best of our knowledge, this is the first study exploring LA strain and SR in CIMR patients before and after surgery.

\section{Conclusions}

In patients with recurrent MR we observed an irreversible impairment of the LA reservoir due to reduced compliance. The filling and contraction phases did not improve after operation and this might be attributable to high LV filling pressure and increased afterload. Left atrial peak global strain, peak systolic strain rate and peak early diastolic strain rate were cofactors associated to recurrent MR.

The assessment of LA strain and strain rate, in addition to other echocardiographic parameters, can be helpful in detecting patients undergoing UMRA who are unlikely to benefit from annuloplasty. 


\section{Appendices}

Appendix 1. Bland -Altman limits of agreement for intra-observer variability.

\begin{tabular}{lccc}
\hline Variable & $\begin{array}{c}\text { Mean } \\
\text { difference }\end{array}$ & $\begin{array}{c}\text { Standard } \\
\text { deviation }\end{array}$ & $\begin{array}{c}\text { 95\% limits } \\
\text { of agreement }\end{array}$ \\
\hline$\varepsilon(\%)$ & 0.92 & 0.07 & $-1.12-1.64$ \\
$\mathrm{SR}_{\mathrm{P}}\left(\mathrm{sec}^{-1}\right)$ & 1.15 & 0.03 & $-1.21-2.04$ \\
$\mathrm{SR}_{\mathrm{E}}\left(\mathrm{sec}^{-1}\right)$ & 1.29 & 0.09 & $-1.20-2.18$ \\
$\mathrm{SR}_{\mathrm{A}}\left(\mathrm{sec}^{-1}\right)$ & 1.61 & 0.10 & $-1.25-2.22$ \\
\hline
\end{tabular}

Observer: Fabiana Lucà (FL) The Bland-Altman method showed excellent agreement. The relative differences were $<5 \%$ for all parameters.

Abbreviations: $\varepsilon$ : Peak systolic strain; $\mathrm{SR}_{\mathrm{P}}$ : Peak systolic strain rate; $\mathrm{SR}_{\mathrm{E}}$ : Peak early diastolic strain rate; $\mathrm{SR}_{\mathrm{A}}$ : peak late diastolic strain rate. 


\section{References}

1. Lamas GA, Mitchell GF, Flaker GC, Smith SC, Jr., Gersh BJ, Basta L, Moye L, Braunwald E, Pfeffer MA. Clinical significance of mitral regurgitation after acute myocardial infarction. Survival and ventricular enlargement investigators. Circulation. 1997;96:827-833

2. Anyanwu A, Rahmanian PB, Filsoufi F, Adams DH. The pathophysiology of ischemic mitral regurgitation: Implications for surgical and percutaneous intervention. J Interven Cardiol. 2006:S78-S86

3. Pierard LA, Carabello BA. Ischaemic mitral regurgitation: Pathophysiology, outcomes and the conundrum of treatment. European heart journal. 2010;31:2996-3005

4. Otsuji Y, Handschumacher MD, Schwammenthal E, Jiang L, Song JK, Guerrero JL, Vlahakes GJ, Levine RA. Insights from three-dimensional echocardiography into the mechanism of functional mitral regurgitation: Direct in vivo demonstration of altered leaflet tethering geometry. Circulation. 1997;96:1999-2008

5. Grigioni F, Enriquez-Sarano M, Zehr KJ, Bailey KR, Tajik AJ. Ischemic mitral regurgitation: Long-term outcome and prognostic implications with quantitative doppler assessment. Circulation. 2001;103:1759-1764

6. Magne J, Pibarot P, Dagenais F, Hachicha Z, Dumesnil JG, Senechal M. Preoperative posterior leaflet angle accurately predicts outcome after restrictive mitral valve annuloplasty for ischemic mitral regurgitation. Circulation. 2007;115:782-791

7. Gelsomino S, Lorusso R, Caciolli S, Capecchi I, Rostagno C, Chioccioli M, De Cicco G, Bille G, Stefano P, Gensini GF. Insights on left ventricular and valvular mechanisms of recurrent ischemic mitral regurgitation after restrictive annuloplasty and coronary artery bypass grafting. The Journal of thoracic and cardiovascular surgery. 2008;136:507-518

8. D'Andrea A, Caso P, Romano S, Scarafile R, Riegler L, Salerno G, Limongelli G, Di Salvo G, Calabro P, Del Viscovo L, Romano G, Maiello C, Santangelo L, Severino S, Cuomo S, Cotrufo M, Calabro R. Different effects of cardiac resynchronization therapy on left atrial function in patients with either idiopathic or ischaemic dilated cardiomyopathy: A two-dimensional speckle strain study. European heart journal. 2007;28:2738-2748

9. Notomi Y, Lysyansky P, Setser RM, Shiota T, Popovic ZB, Martin-Miklovic MG, Weaver JA, Oryszak SJ, Greenberg NL, White RD, Thomas JD. Measurement of ventricular torsion by two-dimensional ultrasound speckle tracking imaging. Journal of the American College of Cardiology. 2005;45:2034-2041

10. Cho GY, Chan J, Leano R, Strudwick M, Marwick TH. Comparison of two-dimensional speckle and tissue velocity based strain and validation with harmonic phase magnetic resonance imaging. The American journal of cardiology. 2006;97:1661-1666

11. Cianciulli TF, Saccheri MC, Lax JA, Bermann AM, Ferreiro DE. Two-dimensional speckle tracking echocardiography for the assessment of atrial function. World journal of cardiology. 2010;2:163-170

12. Zoghbi WA, Enriquez-Sarano M, Foster E, Grayburn PA, Kraft CD, Levine RA, Nihoyannopoulos P, Otto CM, Quinones MA, Rakowski H, Stewart WJ, Waggoner A, Weissman NJ. Recommendations for evaluation of the severity of native valvular regurgitation with two-dimensional and doppler echocardiography. Journal of the American Society of Echocardiography: official publication of the American Society of Echocardiography. 2003;16:777-802

13. Schiller NB, Shah PM, Crawford M, DeMaria A, Devereux R, Feigenbaum H, Gutgesell H, Reichek N, Sahn D, Schnittger I, et al. Recommendations for quantitation of the left ventricle by two-dimensional echocardiography. American society of echocardiography committee on standards, subcommittee on quantitation of two-dimensional echocardiograms. Journal of the American Society of Echocardiography: official publication of the American Society of Echocardiography. 1989;2:358-367

14. Kono T, Sabbah HN, Rosman H, Alam M, Jafri S, Goldstein S. Left ventricular shape is the primary determinant of functional mitral regurgitation in heart failure. Journal of the American College of Cardiology. 1992;20:1594-1598 
15. Cerqueira MD, Weissman NJ, Dilsizian V, Jacobs AK, Kaul S, Laskey WK, Pennell DJ, Rumberger JA, Ryan T, Verani MS. Standardized myocardial segmentation and nomenclature for tomographic imaging of the heart: A statement for healthcare professionals from the cardiac imaging committee of the council on clinical cardiology of the american heart association. Circulation. 2002;105:539-542

16. Yu CM, Sanderson JE, Marwick TH, Oh JK. Tissue doppler imaging a new prognosticator for cardiovascular diseases. Journal of the American College of Cardiology. 2007;49:1903-1914

17. Nagueh SF, Appleton CP, Gillebert TC, Marino PN, Oh JK, Smiseth OA, Waggoner AD, Flachskampf FA, Pellikka PA, Evangelista A. Recommendations for the evaluation of left ventricular diastolic function by echocardiography. Journal of the American Society of Echocardiography: official publication of the American Society of Echocardiography. 2009;22:107-133

18. Borg AN, Pearce KA, Williams SG, Ray SG. Left atrial function and deformation in chronic primary mitral regurgitation. European journal of echocardiography: the journal of the Working Group on Echocardiography of the European Society of Cardiology. 2009;10:833-840

19. Lang RM, Bierig M, Devereux RB, Flachskampf FA, Foster E, Pellikka PA, Picard MH, Roman MJ, Seward J, Shanewise J, Solomon S, Spencer KT, St John Sutton M, Stewart W. Recommendations for chamber quantification. European journal of echocardiography: the journal of the Working Group on Echocardiography of the European Society of Cardiology. 2006;7:79-108

20. Benjamini $Y$, Hochberg Y. Controlling the false discovery rate: A practical and powerful approach to multiple testing. J. R. Statist. Soc. 1995;57:289-300

21. Gelsomino S, Lorusso R, Bille G, Rostagno C, De Cicco G, Romagnoli S, Porciani C, Tetta C, Stefano P, Gensini GF. Left ventricular diastolic function after restrictive mitral ring annuloplasty in chronic ischemic mitral regurgitation and its predictive value on outcome and recurrence of regurgitation. International journal of cardiology. 2009;132:419-428

22. Sirbu C, Herbots L, D'Hooge J, Claus P, Marciniak A, Langeland T, Bijnens B, Rademakers FE, Sutherland GR. Feasibility of strain and strain rate imaging for the assessment of regional left atrial deformation: $A$ study in normal subjects. European journal of echocardiography: the journal of the Working Group on Echocardiography of the European Society of Cardiology. 2006;7:199-208

23. Di Salvo G, Russo MG, Paladini D, Felicetti M, Castaldi B, Tartaglione A, di Pietto L, Ricci C, Morelli C, Pacileo G, Calabro R. Two-dimensional strain to assess regional left and right ventricular longitudinal function in 100 normal foetuses. European journal of echocardiography: the journal of the Working Group on Echocardiography of the European Society of Cardiology. 2008;9:754-756

24. Saraiva RM, Demirkol S, Buakhamsri A, Greenberg N, Popovic ZB, Thomas JD, Klein AL. Left atrial strain measured by two-dimensional speckle tracking represents a new tool to evaluate left atrial function. Journal of the American Society of Echocardiography: official publication of the American Society of Echocardiography. 2010;23:172-180

25. Kihara Y, Sasayama S, Miyazaki S, Onodera T, Susawa T, Nakamura Y, Fujiwara H, Kawai C. Role of the left atrium in adaptation of the heart to chronic mitral regurgitation in conscious dogs. Circulation research. 1988;62:543-553

26. Verheule S, Wilson E, Everett Tt, Shanbhag S, Golden C, Olgin J. Alterations in atrial electrophysiology and tissue structure in a canine model of chronic atrial dilatation due to mitral regurgitation. Circulation. 2003;107:2615-2622

27. Katayama K, Tajimi T, Guth BD, Matsuzaki M, Lee JD, Seitelberger R, Peterson KL. Early diastolic filling dynamics during experimental mitral regurgitation in the conscious dog. Circulation. 1988;78:390-400

28. Sadaniantz A, Miller G, Hadi BJ, Parisi AF. Effects of left ventricular systolic function on left ventricular diastolic filling patterns in severe mitral regurgitation. The American journal of cardiology. 1997;79:1488-1492

29. Appleton CP, Hatle LK, Popp RL. Relation of transmitral flow velocity patterns to left ventricular diastolic function: New insights from a combined hemodynamic and doppler echocardiographic study. Journal of the American College of Cardiology. 1988;12:426-440 
30. Jeevanantham V, Ntim W, Navaneethan SD, Shah S, Johnson AC, Hall B, Shah A, Hundley WG, Daubert $J P$, Fitzgerald D. Meta-analysis of the effect of radiofrequency catheter ablation on left atrial size, volumes and function in patients with atrial fibrillation. The American journal of cardiology. 2010;105:1317-1326

31. Maltais S, Schaff HV, Daly RC, Suri RM, Dearani JA, Sundt TM, 3rd, Enriquez-Sarano M, Topilsky Y, Park SJ. Mitral regurgitation surgery in patients with ischemic cardiomyopathy and ischemic mitral regurgitation: Factors that influence survival. The Journal of thoracic and cardiovascular surgery. 2011;142:995-1001

32. Gelsomino S, Van Garsse L, Luca F, Lorusso R, Cheriex E, Rao CM, Caciolli S, Vizzardi E, Crudeli E, Stefano $P$, Gensini GF, Maessen J. Impact of preoperative anterior leaflet tethering on the recurrence of ischemic mitral regurgitation and the lack of left ventricular reverse remodeling after restrictive annuloplasty. Journal of the American Society of Echocardiography: official publication of the American Society of Echocardiography. 2011;24:1365-1375

33. Bax JJ, Braun J, Somer ST, Klautz R, Holman ER, Versteegh MI, Boersma E, Schalij MJ, van der Wall EE, Dion RA. Restrictive annuloplasty and coronary revascularization in ischemic mitral regurgitation results in reverse left ventricular remodeling. Circulation. 2004;110:II103-II108

34. Geidel S, Lass M, Schneider C, Groth G, Boczor S, Kuck KH, Ostermeyer J. Downsizing of the mitral valve and coronary revascularization in severe ischemic mitral regurgitation results in reverse left ventricular and left atrial remodeling. European journal of cardio-thoracic surgery: official journal of the European Association for Cardio-thoracic Surgery. 2005;27:1011-1016

35. Gelsomino S, Lorusso R, De Cicco G, Capecchi I, Rostagno C, Caciolli S, Romagnoli S, Da Broi U, Stefano $P$, Gensini GF. Five-year echocardiographic results of combined undersized mitral ring annuloplasty and coronary artery bypass grafting for chronic ischaemic mitral regurgitation. European heart journal. 2008;29:231-240 

Chapter 9

\section{Discussion}

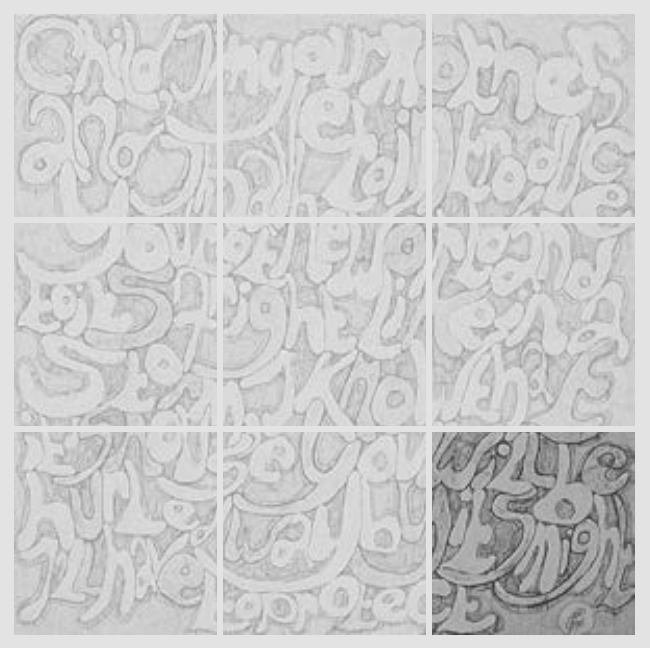


Chronic ischemic mitral regurgitation (CIMR) is a common complication of coronary artery disease that doubles late mortality ${ }^{1,2}$. During the last decades, patients with chronic ischemic mitral regurgitation (CIMR) have been treated by restrictive annuloplasty $(R A)$ in combination with coronary bypass grafting (CABG) ${ }^{3}$. Despite the high initial surgical success rate and positive effects on survival and functional symptomatic status $^{4-7}$, an increasing number of studies report a recurrence of mitral regurgitation $(\mathrm{MR})^{7-10}$, with an incidence up to $35 \%$ and $72 \%$ at 3 - and 5 year-follow up (MR $\geq 2+$ ), respectively ${ }^{7}$. The mechanisms of recurrent $M R$ are the continuous global ${ }^{11,12}$ and local $^{13-16}$ adverse remodeling of the left ventricle, leading to recurrence of mitral leaflet tethering and consequently hindering of the systolic leaflet closure.

Occurrence of recurrent MR in CIMR patients is a much more precise indicator of procedural success than freedom from reoperation. The latter often overestimates the positive results, since many patients with recurrent MR will not undergo reoperation for several reasons, such as good functional class, advanced age, poor left ventricular function or the presence of more co-morbidities. However, recurrent MR should not be confused with residual MR which can be detected by post-bypass transesophageal echocardiography or pre-discharge transthoracic echocardiography ${ }^{17}$. In our studies we excluded patients with residual MR since the procedural outcome should reflect failure of the technique employed.

When evaluating potential predicting factors of recurrent MR (the subject of this thesis), we can describe predictors that reflect systolic LV dysfunction (leaflet geometry, PM function and LV local and global geometry) and predictors that reflect diastolic LV dysfunction (left atrial function). The predictors of LV systolic dysfunction are variables that contribute to the vicious circle of CIMR (FIGURE 1, 2). In contrast, the characteristics of LA dysfunction identify patients with advanced LV ischemic disease and may not have any direct causal association with recurrent MR. However, all predictors find their origin in LV dysfunction since the etiology of CIMR is ischemic myocardial disease. In these patients, MR is functional and reflects a diseased (ischemic) ventricle rather than a disease of the mitral leaflets. 


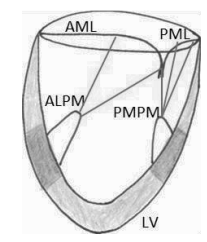

B

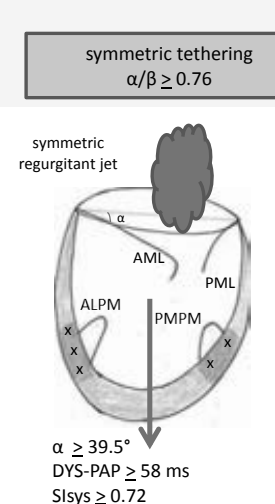

CIMR asymmetric tethering $\alpha / \beta<0.76$

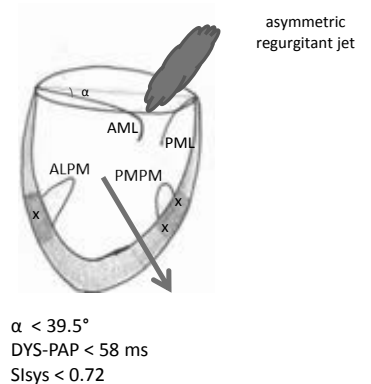

Figure 1. Mitral valve complex (mid-systole) in normal patients $(A)$ and in patients with Chronic ischemic mitral regurgitation (B), divided in two groups based on leaflet tethering pattern.

Abbreviations: $\mathrm{CIMR}=$ Chronic ischemic mitral regurgitation, $\mathrm{AML}=$ anterior mitral leaflet, $\mathrm{PML}=$ posterior mitral leaflet, $\mathrm{ALPM}=$ anterolateral papillary muscle, $\mathrm{PMPM}=$ posteromedial papillary muscle, $\mathrm{LV}=\mathrm{left}$ ventricle, $\alpha=$ anterior mitral leaflet tethering angle, $\beta=$ posterior mitral leaflet tethering angle, DYS-PAP = papillary muscle dyssynchrony, $\mathrm{SI}_{\mathrm{sys}}=$ systolic sphericity index, ALPM-LS = anterolateral papillary muscle longitudinal strain, PMPM-LS = posteromedial papillary muscle longitudinal strain, $\mathrm{GLS}_{\text {peak }}=$ peak global longitudinal strain, ALPM-WMSI = anterolateral papillary muscle wall motion score index (which is the WMSI of left ventricular segments adjacent to ALPM), PMPM-WMSI = posteromedial papillary muscle wall motion score index (which is the WMSI of left ventricular segments adjacent to PMPM). Arrow = resultant tethering vector. 
B

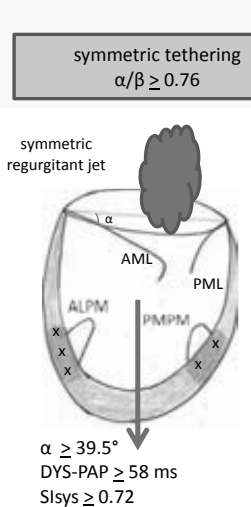

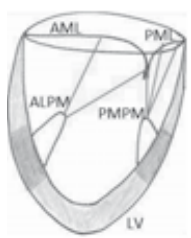

CIMR asymmetric tethering $\alpha / \beta<0.76$

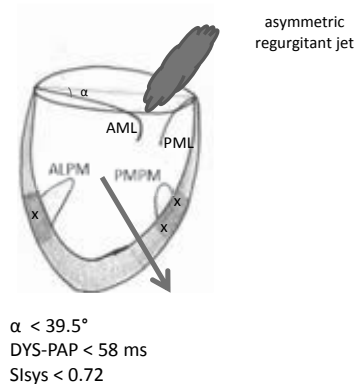

Figure 1. Mitral valve complex (mid-systole) in normal patients $(A)$ and in patients with Chronic ischemic mitral regurgitation (B), divided in two groups based on leaflet tethering pattern.

Abbreviations: $\mathrm{CIMR}=$ Chronic ischemic mitral regurgitation, $\mathrm{AML}=$ anterior mitral leaflet, $\mathrm{PML}=$ posterior mitral leaflet, $\mathrm{ALPM}=$ anterolateral papillary muscle, $\mathrm{PMPM}=$ posteromedial papillary muscle, $\mathrm{LV}=\mathrm{left}$ ventricle, $\alpha=$ anterior mitral leaflet tethering angle, $\beta=$ posterior mitral leaflet tethering angle, DYS-PAP = papillary muscle dyssynchrony, $\mathrm{SI}_{\mathrm{sys}}=$ systolic sphericity index, ALPM-LS = anterolateral papillary muscle longitudinal strain, PMPM-LS = posteromedial papillary muscle longitudinal strain, $\mathrm{GLS}_{\text {peak }}=$ peak global longitudinal strain, ALPM-WMSI = anterolateral papillary muscle wall motion score index (which is the WMSI of left ventricular segments adjacent to ALPM), PMPM-WMSI = posteromedial papillary muscle wall motion score index (which is the WMSI of left ventricular segments adjacent to PMPM). Arrow = resultant tethering vector. 
B

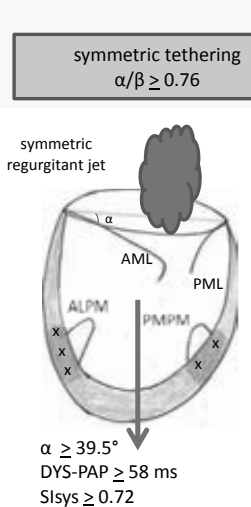

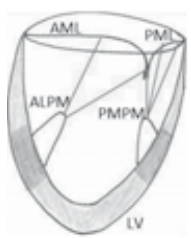

CIMR asymmetric tethering $\alpha / \beta<0.76$

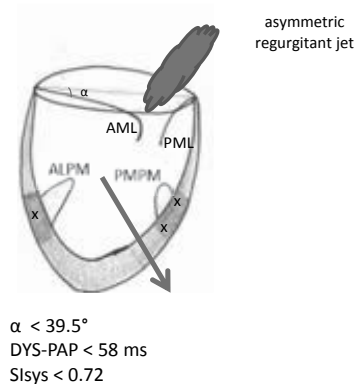

Figure 1. Mitral valve complex (mid-systole) in normal patients $(A)$ and in patients with Chronic ischemic mitral regurgitation (B), divided in two groups based on leaflet tethering pattern.

Abbreviations: $\mathrm{CIMR}=$ Chronic ischemic mitral regurgitation, $\mathrm{AML}=$ anterior mitral leaflet, $\mathrm{PML}=$ posterior mitral leaflet, $\mathrm{ALPM}=$ anterolateral papillary muscle, $\mathrm{PMPM}=$ posteromedial papillary muscle, $\mathrm{LV}=\mathrm{left}$ ventricle, $\alpha=$ anterior mitral leaflet tethering angle, $\beta=$ posterior mitral leaflet tethering angle, DYS-PAP = papillary muscle dyssynchrony, $\mathrm{SI}_{\mathrm{sys}}=$ systolic sphericity index, ALPM-LS = anterolateral papillary muscle longitudinal strain, PMPM-LS = posteromedial papillary muscle longitudinal strain, $\mathrm{GLS}_{\text {peak }}=$ peak global longitudinal strain, ALPM-WMSI = anterolateral papillary muscle wall motion score index (which is the WMSI of left ventricular segments adjacent to ALPM), PMPM-WMSI = posteromedial papillary muscle wall motion score index (which is the WMSI of left ventricular segments adjacent to PMPM). Arrow = resultant tethering vector. 
B

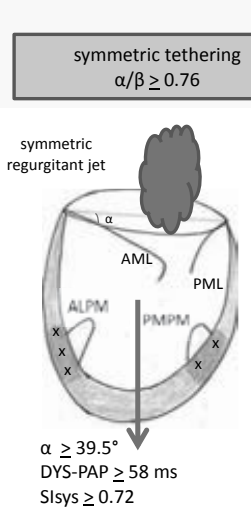

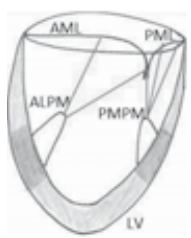

CIMR asymmetric tethering $\alpha / \beta<0.76$

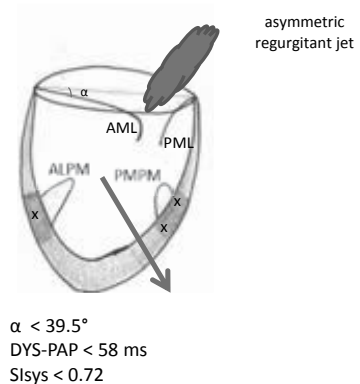

Figure 1. Mitral valve complex (mid-systole) in normal patients $(A)$ and in patients with Chronic ischemic mitral regurgitation (B), divided in two groups based on leaflet tethering pattern.

Abbreviations: $\mathrm{CIMR}=$ Chronic ischemic mitral regurgitation, $\mathrm{AML}=$ anterior mitral leaflet, $\mathrm{PML}=$ posterior mitral leaflet, $\mathrm{ALPM}=$ anterolateral papillary muscle, $\mathrm{PMPM}=$ posteromedial papillary muscle, $\mathrm{LV}=\mathrm{left}$ ventricle, $\alpha=$ anterior mitral leaflet tethering angle, $\beta=$ posterior mitral leaflet tethering angle, DYS-PAP = papillary muscle dyssynchrony, $\mathrm{SI}_{\mathrm{sys}}=$ systolic sphericity index, ALPM-LS = anterolateral papillary muscle longitudinal strain, PMPM-LS = posteromedial papillary muscle longitudinal strain, $\mathrm{GLS}_{\text {peak }}=$ peak global longitudinal strain, ALPM-WMSI = anterolateral papillary muscle wall motion score index (which is the WMSI of left ventricular segments adjacent to ALPM), PMPM-WMSI = posteromedial papillary muscle wall motion score index (which is the WMSI of left ventricular segments adjacent to PMPM). Arrow = resultant tethering vector. 


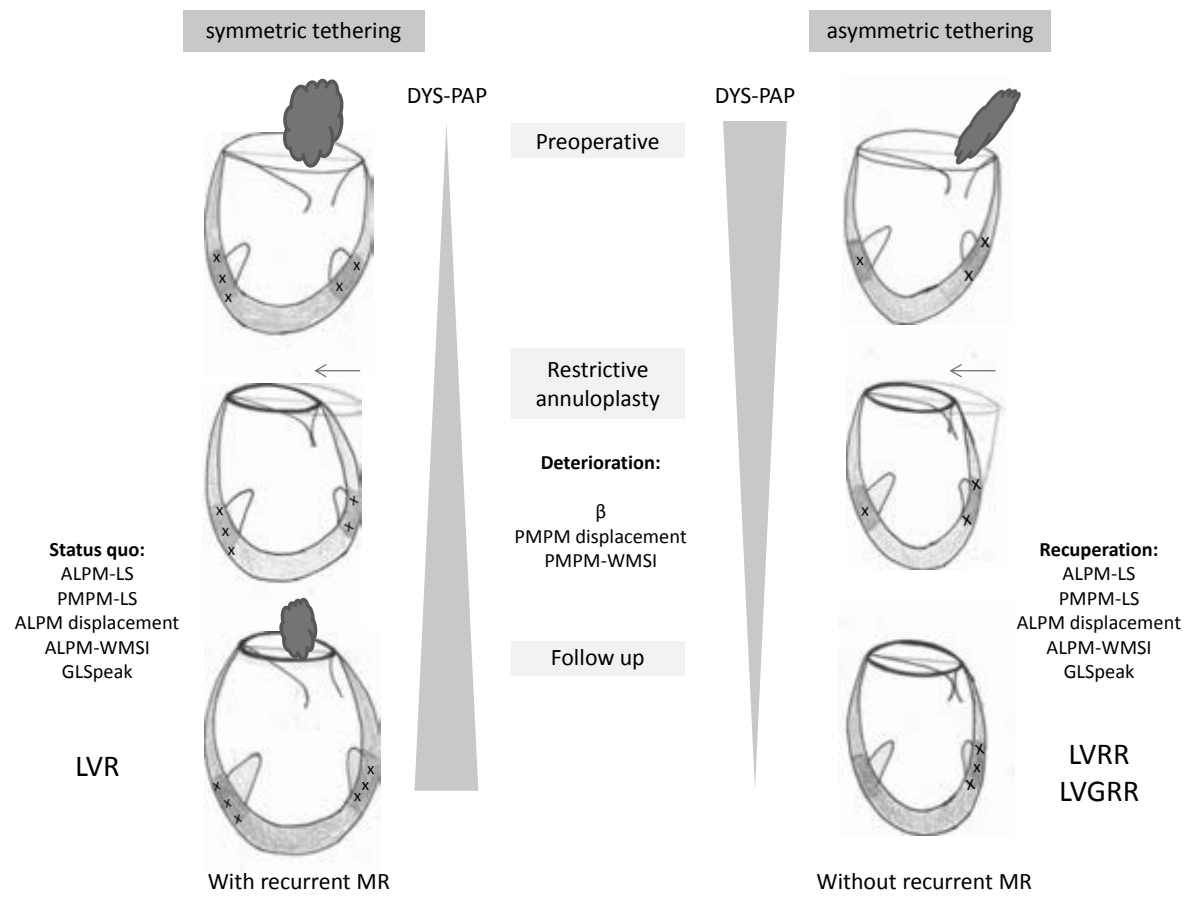

Figure 2. Configuration of the mitral valve complex at baseline and follow up (after restrictive annuloplasty) in patients with chronic ischemic mitral regurgitation, based on mitral leaflet tethering pattern at baseline. Abbreviations: $\mathrm{MR}=$ mitral regurgitation, $\mathrm{AML}=$ anterior mitral leaflet, $\mathrm{PML}=$ posterior mitral leaflet, $\mathrm{ALPM}$ $=$ anterolateral papillary muscle, $\mathrm{PMPM}=$ posteromedial papillary muscle, $\beta=$ posterior mitral leaflet tethering angle, DYS-PAP = papillary muscle dyssynchrony, ALPM-LS = anterolateral papillary muscle longitudinal strain, PMPM-LS = posteromedial papillary muscle longitudinal strain, $\mathrm{GLS}_{\text {peak }}=$ peak global longitudinal strain, LVR = left ventricular remodeling, LVRR = left ventricular reverse remodeling, LVGRR = left ventricular geometrical reverse remodeling, ALPM-WMSI = anterolateral papillary muscle wall motion score index (which is the WMSI of left ventricular segments adjacent to ALPM), PMPM-WMSI = posteromedial papillary muscle wall motion score index (which is the WMSI of left ventricular segments adjacent to PMPM). + indicates the value of WMSI in the LV segments.

\section{Configuration of mitral leaflets as predictor of recurrent MR}

\section{Anterior mitral leaflet angle predicts recurrent MR after RA (FIGURE 1)}

Recently, attention has been drawn to the preoperative mitral valve leaflet configuration to predict MR recurrence and procedural outcomes. Nevertheless, published data are conflicting: recurrence of MR was associated with increased preoperative tethering of the posterior mitral leaflet $(\mathrm{PML})^{14,18}$ or tethering of both leaflets ${ }^{19}$. Magne and coworkers demonstrated that a tethering angle of the $\mathrm{PML} \geq 45^{\circ}$ was associated with persistence of MR and a reduced 3-year event-free survival ${ }^{18}$. However, residual MR 
should be excluded when evaluating outcome of mitral valve repair and the procedural success should not be interpreted by event-free survival but by long term follow up echocardiographic measurements ${ }^{17}$. Kuwahara and colleagues observed an increased PML tethering at follow up and demonstrated a significant contribution of progressive $\mathrm{PML}$ tethering for recurrent/persistent ischemic $M R^{14}$. Indeed, tethering of the $\mathrm{PML}$ is clearly exacerbated by RA: while reducing the septal-lateral annular diameter, the posterior (muscular) annulus is shifted anteriorly towards the fibrous anterior annulus (FIGURE 2). This can deceptively give the impression that the increased PML angle is the predictor of recurrent MR. Green et al. reported in an ovine model, that restrictive annuloplasty produced a markedly impaired motion of a "frozen" PML, transforming the mitral valve (MV) in a mono-cuspid valve where the closure mechanism is only performed by the anterior mitral leaflet $(A M L)^{20}$. Gelsomino and coworkers demonstrated that the tethering angle of the $A M L \geq 39.5^{\circ}$ predicted recurrent $M R^{13}$. In addition, we have reported (Chapter 3 ) that compared to patients with $\mathrm{PML} \geq 45^{\circ}$, those with $A L M \geq 39.5^{\circ}$ had a more than 3-fold higher risk of recurrent $\mathrm{MR}^{21}$.

\section{Angle of the AML: basal or distal}

Lee et al. reported that rendering postoperative mitral competence was highly dependent on the distal anterior leaflet mobility and that the recurrence of MR was predicted by a preoperative distal $A M L$ angle $>25^{\circ}{ }^{22}$. These authors differentiated the $\mathrm{AML}$ angle measurement between the basal and distal (tip) angle, in accordance with the insertion of the secondary and marginal chordae, respectively. They argued that by measuring only the basal angle, the mobility of the whole AML was not taken into account. They explained that the distal AML could be less restricted and might be pivoting around the "knee" (the point where these secondary chordae were attached), which consequently resulted in an alteration of the AML in a concave configuration.

However, it seems to be the basal part of the leaflets that contributes most to the coaptation height $(\mathrm{CH})$ of the $\mathrm{MV}$. In several studies a $\mathrm{CH} \geq 11 \mathrm{~mm}$ is described to be an independent predictor of recurrent $\mathrm{MR}^{13,23}$. Moreover, Messas et al. showed that second-chordal cutting of the AML could reduce MR in IMR, suggesting that AML tethering at this point (second chorda insertion) determined the $M R^{24}$. The importance of the leaflet configuration by tethering on the second-order chordae is demonstrated in several porcine studies on the physiological function of the chordae: van Rijk-Zwikker et al. demonstrated that the AML second-order chordae remained taut during the whole cardiac cycle ${ }^{25}$. Moreover, the forces on the second-order AML chordae were 3fold higher than those on the first-order chordae $\left(0.7 \mathrm{~N}\right.$ vs. $0.2 \mathrm{~N}$ respectively) ${ }^{26}$. Since in CIMR patients MR is caused by left ventricular remodeling (with increased LV dimensions), the tethering forces on the chordae are increased, especially on the secondorder chordae. These findings suggest that measurements based on the angle of the 
AML tip will probably underestimate the recurrence of MR. For this reason, in our studies the AML angle is measured at the basal part of the leaflet.

As described by Messas et al. the decreased progression of LVR in animals with CIMR by second-order chordal cutting ${ }^{27}$ can be explained by the reduction of MR (since the most tethered chordae, responsible for $M R$, are cut) and consequently by the deceleration of the vicious circle of CIMR (chapter 1). Since it hasn't been demonstrated that second-order cutting leads to left ventricular reversed remodeling (LVRR), this procedure cannot be assumed as curative in CIMR.

\section{The AML tethering angle is a primary predictor of MR}

Because of the importance of AML function after RA, Gelsomino and colleagues investigated the role of the preoperative AML mobility in predicting the outcome after surgery $^{13}$. They demonstrated that the preoperative basal AML angle (with a cut off: $\alpha \geq$ $39.5^{\circ}$ ) and the basal $A M L$ excursion angle (with a cut off: $\alpha E X \leq 35^{\circ}$ ) were independent predictors of recurrent MR, while the preoperative PML was not significantly different between patient groups with and without postoperative recurrent MR. However, the mechanism of recurrent MR is the ongoing $L V$ remodeling ${ }^{12}$, or the lack of $L V$ reverse remodeling (LVRR) after $R A^{11}$. Nonetheless, it could be possible that the altered $A M L$ geometry and recurrent MR are both the result of abnormal LV function and geometry in predicting recurrent MR and consequently, that the abnormal LV function and geometry are the primary predictors of MR. In a large multicenter study, we confirmed the importance of $A M L$ tethering in predicting $M R$ (Chapter 2$)^{28}$. Indeed, compared with patients with $P M L$ tethering $\geq 45^{\circ}$, those with severe $A M L$ tethering $\left(>40^{\circ}\right)$ had 3.7 times higher odds of MR recurrence. However, we failed to find any interaction between the AML tethering and other predicting variables of abnormal LV function and global geometrical characteristics (systolic sphericity index $\geq 0.7$; end systolic volume $\geq 145 \mathrm{ml}$; myocardial performance index $\geq 0.9$; wall motion score index $\geq 1.5$ and coaptation height $\geq 11 \mathrm{~mm}$ ). Thus we demonstrated that the AML tethering is a primary independent predictor of MR, not secondary to LV function and geometry.

\section{$L V$ remodeling, the mechanism of recurrent $M R$ in relation to the configuration of mitral leaflets}

$L V$ remodeling $(L V R)^{12}$ or lack of $L V$ reverse remodeling $(L V R R)^{11}$ after $R A$ results in a significant recurrence of MR in CIMR patients. Published data are conflicting whether the recurrent MR is caused by global LV remodeling ${ }^{12}$ or by local remodeling of de LV segments supporting the papillary muscles ${ }^{13-16}$. We explored the relationship between the AML tethering angle (primary predictor of recurrent MR) and the global and local 
LV remodeling (mechanism of recurrent $M R$ ). We investigated whether the leaflet configuration could predict LV remodeling (FIGURE 2).

\section{AML tethering angle predicts lack of global LV reverse remodeling (LVRR)}

Bax and colleagues reported that the high success of CABG and RA, as a treatment for patients with CIMR, was the result of the LVRR after the procedure ${ }^{4}$. The mechanism of LVRR after RA is unclear and explained by the contribution of 1) decreased LV wall stress by remodeling the base of the heart and restoring a more elliptical shape to the LV cavity ${ }^{29}, 2$ ) even a small reduction of LV workload and passive ventricular restraint, which is sufficient to attenuate LV dilation (and progressive functional decline) ${ }^{30}$ or 3 ) decreased LV afterload after reduction in volume overload (reduction of end-systolic wall stress) ${ }^{31}$. Correspondingly, Gelsomino et al. demonstrated that the lack of LVRR after RA led to a significant recurrence of $M R^{11}$. The predictors of LVRR were systolic sphericity index $\left(\mathrm{SI}_{S}<0.72\right)$, myocardial performance index $(\mathrm{MPI}<0.9)$ and wall motion score index (WMSI < 1.59). They excluded patients with residual MR, suggesting that an initial reduction of volume overload (competency of the mitral valve) was not sufficient to induce the LVRR.

In our study (Chapter 3), we investigated the relationship between the preoperative $A M L$ tethering angle and the postoperative reduction in LV volume and geometrical alterations. We demonstrated that an increased $A M L$ tethering (cut-off $\geq 39.5^{\circ}$ ) predicts the recurrence of $M R$, confirming previous studies ${ }^{13,28}$, and the lack of LVRR $^{21}$. LVRR was defined as reduction in the end-systolic volume index $>15 \%$ at follow up (median 14.3 months) compared with the baseline volume index (FIGURE 2). However, also the ventricular shape, which represents global LV remodeling and is defined as the sphericity of the LV, is correlated with the onset of $M R^{32}$. We showed that in patients with an increased $A M L$ angle, the sphericity indices increased significantly at follow up, whereas these indices decreased in patients with a small AML angle. We demonstrated that an increased AML angle predicts the lack of $\boldsymbol{L V}$ geometrical reverse remodeling (LVGRR) ${ }^{21}$ (FIGURE 2). Moreover, compared to patients with a $P M L$ angle $\geq 45^{\circ}$, those patients with an AML angle $\geq 39.5^{\circ}$ showed a more than 4 -fold higher risk of lack of LVRR and LVGRR.

\section{AML tethering and papillary muscle displacement (local LV remodeling)}

Previous studies demonstrated that local remodeling of the LV segments supporting the papillary muscles is a necessary condition for the development of $\mathrm{MR}^{13-16}$. Local remodeling can be described by papillary muscle displacement and separation and by the wall motion score index of the adjacent LV wall (ALPM-WMSI and PMPM-WMSI) (the latter will be discussed in the session on papillary muscle dyssynchrony). 
Gelsomino and coworkers demonstrated that in CIMR patients with a lack of LVRR after surgery, baseline papillary muscle separation and lateral and posterior displacement of the ALPM was significantly increased compared to patients with LVRR ${ }^{11}$. The latter group showed a reduction of the PM separation and displacement of both PMs at follow up, whereas in the group without LVRR all PM displacement variables deteriorated.

In our study (Chapter 3), we demonstrated that in patients with severe AML tethering (large $A M L$ angle) the PMs were significantly separated, reflecting more severe global LV remodeling, compared to patients with a small AML angle ${ }^{21}$. Looking at local remodeling, in the large AML angle group, we found more severe displacement (posterior, lateral and apical) of the ALPM compared to patients with a small AML angle (although significantly displaced compared to controls), whereas the displacement of the PMPM was similar (and significantly increased compared to controls) for both groups of patients. Only the apical PMPM displacement was more severe for patients with a large AML angle.

Apparently it is mainly ALPM displacement that determines the difference in the AML configuration in patients with CIMR. Nevertheless, each PM supplies chordae to both leaflets ${ }^{33}$ and thus displacement of each of the PMs might result in tethering of both leaflets. It is the resultant vector of the PMPM and ALPM displacement that determines the final effect on each leaflet tethering. In our study (Chapter 3 ) the ALPM was mainly displaced apically in the large ALM angle group, resulting in a more apical resultant tethering vector on both leaflets (FIGURE 1). However, it seems that the effect on the PML angle by ALPM displacement is not significantly appreciated in this population (with significant PMPM and ALPM displacement compared to controls).

This can be explained by the morphological characteristics of the mitral valve complex. First, the normal mitral annulus is saddle shaped, but flattens in patients with $\mathrm{CIMR}^{34,35}$. The mitral annulus is composed of a fibrous (anterior) and muscular (posterior) portion. Annular flattening will take place in particular at the muscular structure (apical displacement of the posterior annulus, which is the insertion of the PML). This could explain why the apical displacement of the ALPM has less influence on the PML tethering. Secondly, another explanation could be the absence of third-order chordae in the AML (which is at the level of the leaflet insertion): simultaneous apical tethering on the first- and third-order chordae might displace leaflet insertion and leaflet freeedge in the same direction, resulting in a lower tethering effect.

However, as a result of the restrictive annuloplasty the posterior annulus shifts more anteriorly, resulting in increased PML tethering (FIGURE 2). It is unclear whether this procedure might also lift the posterior annulus to the base. Consequently, some authors advise the use of non-planner RA rings to correct the mitral saddle shape ${ }^{35}$. However, the flattening of the mitral annulus might be a sign of ischemic MR (and a contributor to the vicious circle of (IMR), but not the primary mechanism. Restrictive annuloplasty rings transform the mitral valve into a mono-cuspid valve, where the 
closing mechanism is only performed by the $\mathrm{AML}^{20}$. It does not seem to be expectable that lifting the posterior annulus by the non-planner RA ring would improve mitral valve closure in all CIMR patients. Patients with a mobile ALM (small AML angle) and flattened posterior mitral annulus, will theoretically benefit most from procedures with non-planner RA rings, to prevent them from AML prolapse and residual MR. In contrast, patients with a large AML angle and flattened posterior mitral annulus will theoretically face the disadvantage of non-planner RA rings. By lifting the posterior annulus, the $A M L$ angle increases with a consequently higher risk of residual MR.

\section{Papillary muscle systolic dyssynchrony}

Functional MR (FMR) and intra-ventricular mechanical dyssynchrony are common findings in patients with heart failure and are associated with a poor prognosis ${ }^{36,37}$. Dyssynchronic contractions of LV segments are the leading causes of functional MR in these patients and are independently related to the severity of functional $\mathrm{MR}^{38}$. Several mechanisms describe the contribution of dyssynchrony to functional MR: 1) dyssynchrony causes an uncoordinated regional LV mechanical activation, altering the uncoordinated mitral leaflet motion and tethering ${ }^{14,16,18,39 ; 2) ~ a ~ p o s i t i v e ~ p r e s s u r e ~ g r a d i e n t ~}$ between LA and LV due to improper timing of AV relaxation and contraction cycles results in altered mitral valve closure ${ }^{40}$ and 3 ) a decrease of LV contraction efficiency and closing forces supports mitral leaflet tenting ${ }^{41}$.

Recent studies have demonstrated the beneficial effect of cardiac resynchronization therapy (CRT), showing the immediate reduction of functional mitral regurgitation $(\mathrm{FMR})^{39,42,43}$, due to the improved coordinated timing of mechanical activation of PM insertion sites and the remote decrease of FMR secondary to LV reverse remodeling ${ }^{39}$.

\section{Papillary muscle systolic dyssynchrony is a predictor of MR}

In our studies (Chapters 4, 5 and 6), we assessed systolic papillary muscle dyssynchrony (DYS-PAP) by two-dimensional speckle-tracking echocardiography (2D-STE) in the apical four-chamber view for the ALPM and apical long-axis view for the PMPM ${ }^{44-}$ 46 , confirming with the methodology of Tigen and coworkers in non-ischemic patients ${ }^{47}$. The average value of peak systolic longitudinal strain was determined for each PM: ALPM-LS and PMPM-LS. The beginning of the QRS complex was used as the reference point and the time to peak (TTP) longitudinal strain was quantified for each PM. For the assessment of DYS-PAP, the absolute difference in TTP between ALPM and PMPM was calculated. The peak global longitudinal strain (GLS) (assessed in the apical 2- and 4-chamber views) was used for the assessment of global LV function. We demonstrated that at multivariable analysis DYS-PAP (with a cut-off $\geq 58 \mathrm{~ms}$ ) and the systolic sphericity index (with a cut-off $\geq 0.72$ ) were predictors of MR in addition to 
AML tethering angle and the tethering symmetry (Chapter 4$)^{45}$ (FIGURE 1,2). At baseline, the DYS-PAP had significantly higher values in patients with recurrent MR (MR+ patients) compared to patients without recurrent MR (MR-patients). At follow up, the DYS-PAP in the MR+ patients deteriorated significantly, whereas it significantly improved in the MR- patients. In the patient population with dilated cardiomyopathy (and EF $<35 \%$ ), the DYS-PAP deteriorated significantly in patients with recurrent MR and remained unchanged in patients without MR (Chapter 5$)^{46}$. Remarkably, in this specific study group (Chapter 5 ) the baseline DYS-PAP in the patients without MR already showed lower values compared to the values shown in the MR-patients of the total CIMR group (Chapter 4$)^{45}$.

\section{LV dyssynchrony or papillary muscle dyssynchrony}

It is important to take a closer look at LV dyssynchrony and papillary muscle dyssynchrony since different measurements in the current literature have to be interpreted. First, some studies are based on tissue Doppler imaging (TDI) to determine the peak velocities of $\mathrm{LV}$ segments ${ }^{37,48}$, others use a speckle tracking radial strain ${ }^{49-51}$ or longitudinal strain ${ }^{44-47}$ (Chapter 4, 5 and 6). Second, the segments to assess dyssynchrony are at the base of the $\mathrm{LV}^{37,48}$, in the middle of the $\mathrm{LV}^{49-51}$, or in the papillary muscles itself $^{44-47}$ (Chapter 4, 5 and 6). Third, the time to the peak velocity curve (time-velocity) or the time to the peak strain curve (time-strain), starting at the beginning of the QRS complex ${ }^{37,44-51}$ is standardly accepted by authors. However, for the data-analysis and calculation of dyssynchrony, Bleeker et al. only included the peak time-velocities that occurred during the aortic ejection phase (between opening and closure of the aortic valve $^{48}$; consequently, segments with a very early peak time-velocity (during the isovolumetric LV contraction) were missing in the analysis. Fourth, dyssynchrony was defined as the difference in time between the first and last peak time-velocity (or peak time-strain) $37,48,51$, or as the difference in time between the peak time-strain of the antero-septal segment and the posterior segment ${ }^{49}$, or as the difference in time between the peak time-strain of the LV segment adjacent to the ALPM and the PMPM ${ }^{50}$, or as the difference in time between the peak time-strain of the ALPM and PMPM ${ }^{44-47}$ (Chapter 4, 5 and 6). These measurements clearly separate LV dyssynchrony from the papillary muscle dyssynchrony. Based on earlier studies, the cause of incomplete ischemic MV closure is crucial to differentiate between papillary muscle (dys-)function and the (dys-)function of the adjacent LV wall ${ }^{52,53}$.

\section{Longitudinal strain of the papillary muscles (PM-LS) and adjacent LV wall function (segmental WMSI) in CIMR patients}

To evaluate the function of the papillary muscle itself, we used longitudinal strain assessment by $2 \mathrm{D}-\mathrm{STE}$, conform the methodology of Tigen and coworkers ${ }^{47}$. For func- 
tional evaluation of the adjacent LV wall, segmental WMSI were used: the basal and mid-posterior and inferior segments for the PMPM (the PMPM-WMSI) and the basal and mid-lateral and anterior segments for the ALPM (the ALPM-WMSI) conform with the segmentation of Cerqueira and coworkers ${ }^{54}$.

We found that the longitudinal strain of both PMs showed less negative (less fiber shortening) values at baseline in patients with recurrent MR, which remained unchanged at follow up. In contrast, patients without recurrent MR showed more negative longitudinal strain values of both PMs at baseline, which improved significantly at follow up (Chapters 4 and 5) ${ }^{45,46}$. At baseline both PMs were significantly displaced (posteriorly, laterally and apically) in all patients compared to controls but comparing both patient groups, only the ALPM showed significantly higher values of displacement (posteriorly, laterally and apically) in the group with recurrent MR. At follow up, only the displacement of the ALPM in the MR-group showed improvement, whereas the PMPM displacement values deteriorated significantly in both patient groups (Chapter $5)^{46}$ (FIGURE 2). Displacement (local geometry) and functional shortening (strain) of PMs are not related. The ALPM shows improvement of dislocation and function at follow up in patients without recurrent MR. In contrast, the PMPM shows larger values of dislocation at follow up in both patient groups, while the PMPM shortening significantly improves in the group without recurrent MR.

Functional evaluation of the adjacent myocardial tissue, revealed at baseline in MR- patients, showed a significantly lower (better) ALPM-WMSI compared to MR+ patients, which postoperatively improved, whereas for MR+ patients the ALPM-WMSI remained unchanged. In contrast, the PMPM-WMSI was impaired at baseline and deteriorated at follow up for both patient groups (Chapter 5$)^{46}$. We can conclude that the function of the adjacent myocardial tissue (PM-WMSI) and the displacement of the PMs show similar changes in both patient groups at baseline and follow up (FIGURE 2).

Apparently, differences between both patient groups can be detected preoperatively by the displacement of the ALPM and function of the adjacent LV wall (the ALPM-WMSI). Interestingly, function of both PMs improves postoperatively (more negative longitudinal strain values) in patients without MR, whereas PMPM displacement and function of the LV wall adjacent to the PMPM further deteriorate. This can be explained by the different measurement techniques. On one hand, assessment of the PM by longitudinal strain is a quantification of the real myocardial fiber contraction. In contrast, WMSI is derived by visual grading of the wall motion of individual segments and is the mean value of the evaluated segments. The annuloplasty with a restrictive ring is resulting in an anterior shift of the posterior mitral annulus: the relation/distance between the tip of the PMPM and the mitral valve annulus enlarges and consequently the tethering of the PML is more pronounced, notwithstanding the presence or absence of recurrent $M^{20}$. Increased tension on the PMPM, tangential to the adjacent myocardial wall, might give the visual impression of reduced contractility 
(hypokinesia), and thus a higher (worse) WMSI. Another explanation is the effective continued local LV remodeling in all patients with CIMR and the difference in outcome (global LVRR or lack of global LVRR) is determined by the reversibility of the LV wall dysfunction adjacent to the ALPM (see section: Tethering pattern and the segmental left ventricular strain and Chapter 7).

\section{Mechanical dyssynchrony or electrical dyssynchrony}

Traditionally the duration of the QRS complex on the surface ECG has been used as a marker of LV dyssynchrony ${ }^{55-59}$. Large clinical trials have demonstrated the sustained benefit of CRT in patients with moderate-to-severe heart failure (NYHA class III or IV, $\mathrm{EF} \leq 35 \%)$, and a widened QRS complex $(\geq 120 \mathrm{~ms})^{55-59}$. However, recent studies have demonstrated that QRS duration is only a weak marker of LV dyssynchrony ${ }^{60-63}$. It was observed that $20 \%$ to $30 \%$ of patients with QRS duration $\geq 120 \mathrm{~ms}$ did not have LV dyssynchrony, which may (partially) explain the lack of response to $\mathrm{CRT}^{61}$. On the other hand, it was demonstrated that $20 \%$ to $50 \%$ of heart failure patients with a narrow QRS complex ( $\leq 120 \mathrm{~ms}$ ) may also exhibit LV mechanical dyssynchrony (measured with TDI), and these patients may benefit from CRT ${ }^{60,61,63}$. We can conclude that electrical (ECG) and mechanical dyssynchrony (measured by TDI or strain) should be considered in a different way. A logical explanation is that QRS duration results from right and left ventricular electrical activation, while the study on mechanical dyssynchrony and incompetence of MV closure is concentrated on the left ventricle only. Interestingly, in our ischemic patient population we had lower values of DYS-PAP ${ }^{44}$ compared to data published in the literature in non-ischemic patients ${ }^{47}$. This might be attributable to the small number of subjects with QRS > $120 \mathrm{~ms}(20 \%)$ in our population. On the other hand, one can question whether mechanical and electrical dyssynchrony should be considered differently in ischemic and non-ischemic patients since cellular conduction can be impaired by ischemia ${ }^{64}$.

\section{Dyssynchrony in CIMR patients}

Myocardial ischemia is one of the major causes of LV dyssynchrony ${ }^{65}$ and one may speculate that correction of ischemia by CABG surgery should resynchronize LV contractions. However, published data show that reduction of ischemia by CABG is associated with resynchronization only in a small percentage of patients with a high degree of preoperative dyssynchrony ${ }^{37}$. Accordingly, in our studies we showed that extensive DYS-PAP could not be reversed by revascularization (Chapters 4 and 5) and we concluded that DYS-PAP might represent a landmark of advanced myocardial damage in CIMR and a sign of irreversible progression of the ischemic disease. Furthermore, it has been demonstrated that improvement from LV dyssynchrony plays an important role in the development of LVRR after $\mathrm{CRT}^{66}$. Therefore, we suppose that by resynchroniza- 
tion, the vicious circle of remodeling is interrupted. Indeed, in our studies, patients with asymmetric tethering (Chapter 6 ) and without severe DYS-PAP at baseline showed significant LVRR and MV competence after the procedure. In contrast, in patients with symmetric tethering, the DYS-PAP was significantly increased at baseline and remained irreversible (FIGURE 2). The lack of resynchronization might reinforce the vicious circle of remodeling and MR (Chapter 1 ).

\section{Symmetric tethering pattern predicts postoperative papillary muscle dyssynchrony}

The concept of the tethering pattern (symmetric or asymmetric) of the MV in CIMR patients was first introduced by Agricola and coworkers ${ }^{67}$ with a predominant posterior tethering of both leaflets in the asymmetric group and a predominant apical tethering of both leaflets in the symmetric group (FIGURE 1). Leaflet tethering reflects the relative displacement of the tip of papillary muscles (PMs) to the annular leaflet inser$\operatorname{tion}^{68,69}$. Since each papillary muscle supplies chordae to both leaflets ${ }^{33}$, the difference in tethering pattern reflects the resultant tethering vector, created by the relative dislocation of the two PM tips to the leaflet insertion at the annulus. The tethering pattern is calculated by the $\operatorname{AML}(\alpha)$ and PML $(\beta)$ tethering angle with their ratio $\alpha / \beta$ being a quantitative measurement of tethering symmetry. Gelsomino and colleagues demonstrated that a symmetric tethering pattern, with a cut-off $\geq 0.76$, was strongly associated with recurrence of MR after RA ${ }^{13}$. Our experience (Chapter 6 ) demonstrated that in patients with preoperative asymmetrical tethering, the GLS, PMPM-LS , ALPMLS and the DYS-PAP significantly improved at follow up ${ }^{44}$. In contrast, in patients with a preoperative symmetrical tethering pattern, the DYS-PAP worsened, whereas the GLS, PMPM-LS and the ALPM-LS were severely impaired and did not improve. The symmetric tethering pattern was the strongest predictor of significant postoperative DYS-PAP, while variables from our earlier studies were confirmed to be predictors of recurrent MR (DYS-PAP $\geq 58 \mathrm{~ms}, \alpha \geq 39.5^{\circ}, \alpha / \beta \geq 0.76$ and systolic sphericity index $\geq 0.72$ ).

It seems that the predictors of recurrent $M R$ ( $A M L$ tethering, symmetric tethering pattern and DYS-PAP) reflect the condition of ALPM displacement and function as well as the disability of recuperation after revascularization and annuloplasty. The different expressions of ischemic disease in the ALPM compared to the PMPM can be explained by the anatomy of their arterial blood supply. Considering the coronary anatomy, the blood supply of the PMPM derives in 63\% from one of the three main coronary arteries, whereas the ALPM is supplied in $71 \%$ by two of the main coronary arteries ${ }^{70}$. As a clinical consequence, myocardial infarction may cause papillary muscle dysfunction and MR when blood supply is provided by one rather than two vessels, as is more frequently the case for the PMPM rather than for the ALPM ${ }^{71}$. This conclusion can be confirmed by the study of Tanimoto, showing infarction of PMPM and ALPM in 77\% 
and $26 \%$, respectively, of patients with acute myocardial infarction. As a consequence, patients with impaired ALPM function are more likely to suffer from a more generalized coronary insufficiency (with at least involvement of two main coronary arteries). One can question whether LV dysfunction is generalized in patients with recurrent MR and more localized in patients without recurrent MR.

\section{Tethering pattern and segmental left ventricular strain}

The DYS-PAP in ischemic patients seems to be irreversible in the MR+ population and deteriorates further after RA ${ }^{45}$. Moreover, advanced DYS-PAP can be defined by tethering symmetry in CIMR patients who are unlikely to benefit from $\mathrm{RA}^{44}$. We investigated (Chapter 7) whether the tethering pattern might be related to generalized or localized LV remodeling, by segmental strain measurements. Global and segmental longitudinal, circumferential and radial strain was measured by 2D-STE. Valve geometrical characteristics, LV volumes and function were assessed and segmental myocardial function adjacent to the PMs was calculated by WMSI. The PMPM-WMSI was increased in both groups and deteriorated at follow up. The ALPM-WMSI was only slightly increased in the asymmetric group and improved, although not significantly, whereas in the symmetric group the ALPM-WMSI was severely increased and remained stable at follow up. In accordance with our findings in Chapter 6, the function (WMSI) of the myocardial wall adjacent to the PMPM seems to be significantly different between the symmetric and asymmetric group, but in both groups this function deteriorates at follow up. In contrast, the function of the myocardium adjacent to the ALPM is significantly impaired in the symmetric group at baseline, but remains constant at follow up $^{44}$. Apparently, the difference in outcome is related to myocardial function adjacent to the ALPM.

Segmental longitudinal strain, circumferential strain and radial strain all show similar variations between both groups: at baseline the symmetric group showed worse values in the anterolateral, anterior and inferolateral segments, whereas the asymmetric group showed over-all better values, although mostly impaired in the infero-septum and the inferior wall (Chapter 7). At one-year follow up all strain values in the asymmetric group improved significantly, whereas in the symmetric group the strain in the inferior, anteroseptal and inferoseptal walls deteriorated, remaining unchanged in the other segments.

Our main finding is that symmetric tethering seems to reflect more generalized disease with significant involvement of all LV segments, whereas patients with asymmetric tethering showed a more localized worsening of strain, located in the area which comprises the insertion of the PMPM. Ischemic MR by localized reverse remodeling has been reported by other authors ${ }^{53}$. Since the tethering pattern reflects the resultant tethering vector of the PMs, we suggest that in asymmetric tethering 
(affected PMPM), the posterior leaflet is simply drawn more inferiorly (and posteriorly) than apically, restricting the PML from reaching its normal coaptation point and shifting the coaptation posteriorly, creating an asymmetric tethering and regurgitant jet. In contrast, in symmetric tethering, it seems that all segments of the LV are involved adding apical tethering. The higher values of the ALPM-WMSI confirmed the hypothesis of additional involvement of the LV segments adjacent to the ALPM. The net result of these forces causes more apical tenting of the leaflets, creating a symmetric regurgitant jet.

Thus, it seems that the segmental LV function adjacent to the ALPM might be helpful in differentiating between generalized and localized LV disease, since at follow up the PMPM-WMSI deteriorates in both groups.

\section{LA function - a reflection of diastolic LV (dys)function in CIMR}

Left atrial (LA) function has been poorly investigated in patients with ischemic dilated heart disease ${ }^{72}$. General functional evaluation by 2D-speckle-tracking echocardiography has been described by Cianciulli ${ }^{73}$ and this thesis contains the first study exploring LA function in CIMR patients before and after surgery ${ }^{74}$, evaluating LA volumes, left atrial peak global strain $(\varepsilon)$, peak systolic strain rate $\left(S_{\mathrm{P}}\right.$ the reservoir phase of $\left.L A\right)$, peak early diastolic strain rate $\left(S R_{E}\right.$ : conduit phase of $\left.L A\right)$ and peak late diastolic strain rate $\left(S R_{A}\right.$ : contractile phase of $\left.L A\right)$ (Chapter 8$)$. Functional evaluation of $L A$ has to be appreciated as a consequence of LA volume load, LA wall compliance and left ventricu$\operatorname{lar}(\mathrm{LV})$ function (relaxation and LV volume load).

\section{LA volume and reservoir function}

In patients with CIMR, LV volume overload is one of the variables of the vicious circle, leading to further LV remodeling and MR. In our study, LV volumes were increased in all patients with CIMR, compared to controls. Because of the mitral regurgitant jet, atrial volumes were significantly higher and the $\varepsilon$ and $\mathrm{SR}_{\mathrm{p}}$ showed higher values which were restored (volumes and function) after annuloplasty by reduction of preload. Interestingly, this volumetric and functional LA behavior was only seen in patients without postoperative recurrent MR (MR-patients). The patients with postoperative MR (MR+ patients) showed smaller volumes and significantly decreased $\varepsilon$ and $\mathrm{SR}_{\mathrm{p}}$ preoperative values that remained unchanged after surgery. This might be explained by irreversible ultra-structural changes of the atrial wall in $\mathrm{MR}+$ patients, resulting in loss of wall compliance, elasticity and/or contraction. The question remains whether this is a consequence of chronic LA volume overload or increased chronic LA pressure (increased LV filling pressure). MR in both populations was comparable (the effective orifice area as well as the regurgitant volume and regurgitant fraction), but the LV 
volumes, the WMSI and geometrical remodeling indices (sphericity indices) were significantly increased in the MR+ population. We assume that loss of LA wall compliance is a consequence of chronic increased filling pressures.

This conclusion is in accordance with previous morphological findings of human vascular "low-pressure" tissue that is exposed to higher pressure conditions: saphenous veins become noncompliant when used as a coronary bypass graft ${ }^{75}$ and the vascular remodeling of the pulmonary artery and veins is triggered by the pressure, per se, in patients with pulmonary hypertension ${ }^{76}$. The consequence of structural LA wall alterations on LA function is well explored in the atrial fibrillation population, where atrial wall fibrosis, diagnosed by delayed-enhancement MRI, was inversely related to LA strain and strain rate ${ }^{77}$. Nevertheless, since the study in Chapter 8 was a retrospective study, structural information of the LA wall (by MRI) was not available ${ }^{74}$. Kokubu et al. studied LA function in patients with arterial hypertension (HT) and found that the LA reservoir phase (mean peak systolic strain rates) had lower values than in patients without HT. Moreover, LA reservoir dysfunction was present before these patients developed left ventricular hypertrophy (LVH) or LA enlargement. Treatment by reninangiotensin system (RAS) inhibitors was only successful (normalization of SR $\mathrm{P}_{\mathrm{P}}$ if the LA was not dilated ${ }^{78}$. We can conclude that left atrial reservoir dysfunction is reversible as long as structural changes of the LA wall are absent.

\section{LA conduit and contractile function}

The reversibility of atrial function in MR- patients could also be appreciated during the conduit phase and active contractile phase as described in Chapter $8^{74}$. Compared to controls passive LV filling was raised in MR- patients because of the increased transmitral gradient in the condition of normal LV relaxation (isovolumetric relaxation time, comparable to controls) and compliant LA wall. The active contraction was supranormal and correlated with the LA reservoir phase (a pre-load dependency of LA function, comparable to optimization of the Frank-Starling mechanism). After surgery, $\mathrm{SR}_{\mathrm{E}}$ and $\mathrm{SR}_{\mathrm{A}}$ were restored to values comparable to the controls. In contrast, LA conduit function and contractile function in MR+ patients were significantly impaired and did not improve after surgery. Spencer et al. reported that the LA conduit phase and active contractile phase were age-dependent ${ }^{79}$. The patient groups (MR+, MR- and the matched control group) in our study did not show significant differences in age. Reduction of $\mathrm{SR}_{\mathrm{E}}$ and $\mathrm{SR}_{\mathrm{A}}$ was caused by an impaired LV diastolic function (impaired LV relaxation with decreased $\mathrm{E}$ wave and DT) and loss of atrial contractility. The latter could be the result of a deteriorated Frank-Starling mechanism or a structural loss of atrial myocytes (atrial fibrosis). However, after surgery, there was no functional improvement of these LA phases, suggesting that this patient population has a condition of ischemic heart disease with irreversible LV function and LA wall non-compliancy. It would be interesting to differentiate between the condition of severely impaired LV function and 
fibrosis of LA. Kuppahalla and coworkers demonstrated that in patients with AF, more LA fibrosis was found in the persistent AF group compared to the paroxysmal group and while the EF (ejection fraction), sex, age and MR were comparable, the persistent group showed an impaired LA strain ${ }^{77}$. They showed that strain measurements could vary independently of LV dysfunction. However, the strain rates were not significantly different.

Our study suggests that LV dysfunction precedes the manifest changes of LA function, leading to LA dysfunction. From Kokubu, we learned that treatment of LV dysfunction restored LA function ${ }^{78}$. Thus we can conclude that the description of LA dysfunction in the CIMR population is a population with irreversible diastolic LV dysfunction and a population with lack of reversed remodeling, even after RA and CABG. The fact that we excluded patients with residual MR in our study shows that the initial preload reduction by temporary MV competence, could not restore or improve LA function, which strengthens the hypothesis that we were dealing with a population with irreversible ischemic disease.

\section{Conclusion and perspectives}

During the last two decades, patients with CIMR have been treated by CABG and restrictive annuloplasty. Recent studies have shown that some of these patients benefit from this procedure while others do not. Searching for predictive factors of recurrent $\mathrm{MR}$, our studies led us to define two different patient populations: patients with local irreversible ischemic disease (but with global functional reversibility) and patients with global irreversible ischemic disease (without functional reversibility).

Local irreversible ischemic disease more specifically involves the inferoseptal and inferior LV segments, the region of the PMPM, with the ALPM region being less involved. However, reversibility of the ventricle occurs mostly in the ALPM region, while the PMPM region will further remodel. Apparently, localized disease (region PMPM) is irreversible, but the general consequences for the ventricle (increased volumes, sphericity indices, WMI, MPI and altered AML geometry compared to controls) can recuperate.

Global irreversible disease is reflected in populations with a general involvement of all LV segments (and compared to local disease, more expressive in the ALPM region). Neither the PMPM region nor the ALPM region will recuperate after the procedure.

One can question whether these two populations are an expression of different ischemic diseases or an expression of different states of ischemic disease. The existence of different pathologies in ischemic coronary artery disease can be appreciated by the presence of small-vessel disease in patients with patent large coronary arteries ${ }^{80}$ and other patients with proximal solitary lesions in one of the three main coronaries ${ }^{81}$. Moreover, ischemic heart disease is considered in a different way in women ${ }^{82-84}$ and in 
patients with and without diabetes mellitus (DM). For example, patients with DM have more multi-vessel disease, which is more progressive and have a higher risk of myocardial infarction and heart failure ${ }^{85-87}$. However, we could not find any difference in patient characteristics in our studies (e.g. DM, sex or number of coronary vessel disease). Thus, we conclude that the difference between our two patient populations is not based on different ischemic diseases.

Ambrose and colleagues evaluated the progression of coronary heart disease by angiography ${ }^{88}$. They showed that myocardial infarction frequently developed from previously non-severe coronary artery lesions. However, they could not predict the onset and location of the myocardial infarction due to these lesions. Apparently, ischemic preconditioning by repetitive ischemic events may prevent or delay cell death after coronary occlusion ${ }^{89}$ and thus the number of diseased coronary arteries are not correlated with the size of myocardial infarction (scar). In our studies, we could not find any difference in the number of diseased coronary arteries or bypass grafts between either patient population. It is possible that patients in the global irreversible group had larger myocardial scar sizes than patients in the local irreversible group, even if the number of diseased coronary arteries and grafts were comparable. However, as explained previously, since our studies were all retrospective, information on scar size was not available. Nonetheless, we observed that patients reflecting global irreversible disease, showed more extensive abnormal, irreversible function in all LV segments, whereas patients displaying localized irreversible disease showed significant dysfunction specifically in the region of the posterior papillary muscle. These findings suggest that the difference between our two patient populations might be based on a different state of ischemic disease.

Another interesting dispute is the presence of viable myocardial tissue in these two populations versus the reversibility of myocardial function. However, viability of myocardium is not the sole condition of improved LV function after surgery ${ }^{90}$. The question of viability in CIMR patients (by MRI), undergoing CABG and RA will be the subject of a randomized trial.

For patients who will not benefit from restrictive annuloplasty, other therapeutic options should be evaluated. Besides different repair techniques at the level of the valve (e.g. Alfieri's edge-to-edge ${ }^{91}$ ), the chordae (e.g. Messa's second-order cutting technique ${ }^{92}$ ), the papillary muscles (e.g. PMPM re-location technique ${ }^{93}$ ) and the left ventricle (e.g. CorCap Cardiac Support Device ${ }^{94}$ ) or valve replacement, one can question for which ischemic patients cardiac resynchronization therapy (CRT) might be the solution. In our discussion we tried to clarify that mechanical and electrical dyssynchrony should be appreciated in a different way. However, in ischemic patients it is not clear which part of mechanical dyssynchrony is caused by conduction disorders (and might be treatable by CRT) or caused by cell-to-cell electrical junctional loss ${ }^{95,96}$ (and might be non-responsive to CRT). Moreover, it is important to stress the difference between LV dyssynchrony and papillary muscle dyssynchrony (see discussion). 
Our data confirm that restrictive annuloplasty can only be effectively employed in selected patients. We provide evidence that these patients can be identified on the basis of clinical and echocardiographic characteristics. RA is indicated in CIMR patients with an echocardiographic asymmetric mitral valve tethering pattern, with an AML tethering angle $<39.5^{\circ}$, SIS $<0.7$ and in the absence of severe DYS-PAP ( $<58 \mathrm{~ms}$ ) and advanced diastolic LV dysfunction (reduced LA function). The role of the presence and location of viable myocardium should be further investigated in a prospective study. 


\section{References}

1. Barzilai B, Gessler C, Jr., Perez JE, Schaab C, Jaffe AS. Significance of doppler-detected mitral regurgitation in acute myocardial infarction. The American journal of cardiology. 1988;61:220-223

2. Lamas GA, Mitchell GF, Flaker GC, Smith SC, Jr., Gersh BJ, Basta L, Moye L, Braunwald E, Pfeffer MA. Clinical significance of mitral regurgitation after acute myocardial infarction. Survival and ventricular enlargement investigators. Circulation. 1997;96:827-833

3. Bolling SF, Pagani FD, Deeb GM, Bach DS. Intermediate-term outcome of mitral reconstruction in cardiomyopathy. The Journal of thoracic and cardiovascular surgery. 1998;115:381-386; discussion 387388

4. Bax JJ, Braun J, Somer ST, Klautz R, Holman ER, Versteegh MI, Boersma E, Schalij MJ, van der Wall EE, Dion RA. Restrictive annuloplasty and coronary revascularization in ischemic mitral regurgitation results in reverse left ventricular remodeling. Circulation. 2004;110:II103-II108

5. Braun J, van de Veire NR, Klautz RJ, Versteegh MI, Holman ER, Westenberg JJ, Boersma E, van der Wall $\mathrm{EE}, \mathrm{Bax} \mathrm{JJ}$, Dion RA. Restrictive mitral annuloplasty cures ischemic mitral regurgitation and heart failure. The Annals of thoracic surgery. 2008;85:430-436; discussion 436-437

6. Geidel S, Lass M, Schneider C, Groth G, Boczor S, Kuck KH, Ostermeyer J. Downsizing of the mitral valve and coronary revascularization in severe ischemic mitral regurgitation results in reverse left ventricular and left atrial remodeling. European journal of cardio-thoracic surgery: official journal of the European Association for Cardio-thoracic Surgery. 2005;27:1011-1016

7. Gelsomino S, Lorusso R, De Cicco G, Capecchi I, Rostagno C, Caciolli S, Romagnoli S, Da Broi U, Stefano $P$, Gensini GF. Five-year echocardiographic results of combined undersized mitral ring annuloplasty and coronary artery bypass grafting for chronic ischaemic mitral regurgitation. European heart journal. 2008;29:231-240

8. Crabtree TD, Bailey MS, Moon MR, Munfakh N, Pasque MK, Lawton JS, Moazami N, Aubuchon KA, AlDadah AS, Damiano RJ, Jr. Recurrent mitral regurgitation and risk factors for early and late mortality after mitral valve repair for functional ischemic mitral regurgitation. The Annals of thoracic surgery. 2008;85:1537-1542; discussion 1542-1543

9. Mihaljevic T, Lam BK, Rajeswaran J, Takagaki M, Lauer MS, Gillinov AM, Blackstone EH, Lytle BW. Impact of mitral valve annuloplasty combined with revascularization in patients with functional ischemic mitral regurgitation. Journal of the American College of Cardiology. 2007;49:2191-2201

10. Serri K, Bouchard D, Demers P, Coutu M, Pellerin M, Carrier M, Perrault LP, Cartier R, Page P, Cossette $\mathrm{M}$, Basmadjian AJ. Is a good perioperative echocardiographic result predictive of durability in ischemic mitral valve repair? The Journal of thoracic and cardiovascular surgery. 2006;131:565-573 e562

11. Gelsomino S, Lorusso R, Capecchi I, Rostagno C, Romagnoli S, Bille G, De Cicco G, Tetta C, Stefano P, Gensini GF. Left ventricular reverse remodeling after undersized mitral ring annuloplasty in patients with ischemic regurgitation. The Annals of thoracic surgery. 2008;85:1319-1330

12. Hung J, Papakostas L, Tahta SA, Hardy BG, Bollen BA, Duran CM, Levine RA. Mechanism of recurrent ischemic mitral regurgitation after annuloplasty: Continued Iv remodeling as a moving target. Circulation. 2004;110:1185-II90

13. Gelsomino S, Lorusso R, Caciolli S, Capecchi I, Rostagno C, Chioccioli M, De Cicco G, Bille G, Stefano P, Gensini GF. Insights on left ventricular and valvular mechanisms of recurrent ischemic mitral regurgitation after restrictive annuloplasty and coronary artery bypass grafting. The Journal of thoracic and cardiovascular surgery. 2008;136:507-518

14. Kuwahara E, Otsuji Y, Iguro Y, Ueno T, Zhu F, Mizukami N, Kubota K, Nakashiki K, Yuasa T, Yu B, Uemura T, Takasaki K, Miyata M, Hamasaki S, Kisanuki A, Levine RA, Sakata R, Tei C. Mechanism of recurrent/persistent ischemic/functional mitral regurgitation in the chronic phase after surgical annuloplasty: Importance of augmented posterior leaflet tethering. Circulation. 2006;114:1529-1534 
15. Magne J, Pibarot P, Dumesnil JG, Senechal M. Continued global left ventricular remodeling is not the sole mechanism responsible for the late recurrence of ischemic mitral regurgitation after restrictive annuloplasty. Journal of the American Society of Echocardiography: official publication of the American Society of Echocardiography. 2009;22:1256-1264

16. Zhu F, Otsuji Y, Yotsumoto G, Yuasa T, Ueno T, Yu B, Koriyama C, Hamasaki S, Biro S, Kisanuki A, Minagoe S, Levine RA, Sakata R, Tei C. Mechanism of persistent ischemic mitral regurgitation after annuloplasty: Importance of augmented posterior mitral leaflet tethering. Circulation. 2005;112:I3961401

17. Adams DH, Anyanwu A. Pitfalls and limitations in measuring and interpreting the outcomes of mitral valve repair. The Journal of thoracic and cardiovascular surgery. 2006;131:523-529

18. Magne J, Pibarot P, Dagenais F, Hachicha Z, Dumesnil JG, Senechal M. Preoperative posterior leaflet angle accurately predicts outcome after restrictive mitral valve annuloplasty for ischemic mitral regurgitation. Circulation. 2007;115:782-791

19. Ciarka A, Braun J, Delgado V, Versteegh M, Boersma E, Klautz R, Dion R, Bax JJ, Van de Veire N. Predictors of mitral regurgitation recurrence in patients with heart failure undergoing mitral valve annuloplasty. The American journal of cardiology. 2010;106:395-401

20. Green GR, Dagum P, Glasson JR, Nistal JF, Daughters GT, 2nd, Ingels NB, Jr., Miller DC. Restricted posterior leaflet motion after mitral ring annuloplasty. The Annals of thoracic surgery. 1999;68:21002106

21. Gelsomino S, Van Garsse L, Luca F, Lorusso R, Cheriex E, Rao CM, Caciolli S, Vizzardi E, Crudeli E, Stefano $P$, Gensini GF, Maessen J. Impact of preoperative anterior leaflet tethering on the recurrence of ischemic mitral regurgitation and the lack of left ventricular reverse remodeling after restrictive annuloplasty. Journal of the American Society of Echocardiography: official publication of the American Society of Echocardiography. 2011;24:1365-1375

22. Lee AP, Acker M, Kubo SH, Bolling SF, Park SW, Bruce CJ, Oh JK. Mechanisms of recurrent functional mitral regurgitation after mitral valve repair in nonischemic dilated cardiomyopathy: Importance of distal anterior leaflet tethering. Circulation. 2009;119:2606-2614

23. Calafiore AM, Di Mauro M, Gallina S, Di Giammarco G, laco AL, Teodori G, Tavarozzi I. Mitral valve surgery for chronic ischemic mitral regurgitation. The Annals of thoracic surgery. 2004;77:1989-1997

24. Messas E, Guerrero JL, Handschumacher MD, Conrad C, Chow CM, Sullivan S, Yoganathan AP, Levine RA. Chordal cutting: A new therapeutic approach for ischemic mitral regurgitation. Circulation. 2001;104:1958-1963

25. van Rijk-Zwikker GL, Delemarre BJ, Huysmans HA. Mitral valve anatomy and morphology: Relevance to mitral valve replacement and valve reconstruction. Journal of cardiac surgery. 1994;9:255-261

26. Lomholt M, Nielsen SL, Hansen SB, Andersen NT, Hasenkam JM. Differential tension between secondary and primary mitral chordae in an acute in-vivo porcine model. The Journal of heart valve disease. 2002;11:337-345

27. Messas E, Bel A, Szymanski C, Cohen I, Touchot B, Handschumacher MD, Desnos M, Carpentier A, Menasche $P$, Hagege AA, Levine RA. Relief of mitral leaflet tethering following chronic myocardial infarction by chordal cutting diminishes left ventricular remodeling. Circulation. Cardiovascular imaging. 2010;3:679-686

28. Van Garsse L, Gelsomino S, Luca F, Lorusso R, Rao CM, Stefano P, Maessen J. Importance of anterior leaflet tethering in predicting recurrence of ischemic mitral regurgitation after restrictive annuloplasty. The Journal of thoracic and cardiovascular surgery. 2012;143:S54-S59

29. Bach DS, Bolling SF. Improvement following correction of secondary mitral regurgitation in end-stage cardiomyopathy with mitral annuloplasty. The American journal of cardiology. 1996;78:966-969

30. Power JM, Raman J, Dornom A, Farish SJ, Burrell LM, Tonkin AM, Buxton B, Alferness CA. Passive ventricular constraint amends the course of heart failure: A study in an ovine model of dilated cardiomyopathy. Cardiovascular research. 1999;44:549-555 
31. Takeda K, Taniguchi K, Shudo Y, Kainuma S, Hamada S, Matsue H, Matsumiya G, Sawa Y. Mechanism of beneficial effects of restrictive mitral annuloplasty in patients with dilated cardiomyopathy and functional mitral regurgitation. Circulation. 2010;122:S3-S9

32. Kono T, Sabbah HN, Rosman H, Alam M, Jafri S, Goldstein S. Left ventricular shape is the primary determinant of functional mitral regurgitation in heart failure. Journal of the American College of Cardiology. 1992;20:1594-1598

33. Victor S, Nayak VM. Variations in the papillary muscles of the normal mitral valve and their surgical relevance. Journal of cardiac surgery. 1995;10:597-607

34. Tibayan FA, Rodriguez F, Langer F, Zasio MK, Bailey L, Liang D, Daughters GT, Ingels NB, Jr., Miller DC. Annular remodeling in chronic ischemic mitral regurgitation: Ring selection implications. The Annals of thoracic surgery. 2003;76:1549-1554; discussion 1554-1545

35. Watanabe N, Ogasawara Y, Yamaura Y, Kawamoto T, Akasaka T, Yoshida K. Geometric deformity of the mitral annulus in patients with ischemic mitral regurgitation: A real-time three-dimensional echocardiographic study. The Journal of heart valve disease. 2005;14:447-452

36. Cho GY, Song JK, Park WJ, Han SW, Choi SH, Doo YC, Oh DJ, Lee Y. Mechanical dyssynchrony assessed by tissue doppler imaging is a powerful predictor of mortality in congestive heart failure with normal qrs duration. Journal of the American College of Cardiology. 2005;46:2237-2243

37. Penicka M, Bartunek J, Lang O, Medilek K, Tousek P, Vanderheyden M, De Bruyne B, Maruskova M, Widimsky $P$. Severe left ventricular dyssynchrony is associated with poor prognosis in patients with moderate systolic heart failure undergoing coronary artery bypass grafting. Journal of the American College of Cardiology. 2007;50:1315-1323

38. Hung CL, Tien SL, Lo Cl, Hung TC, Yeh HI, Wang YS. The incremental value of regional dyssynchrony in determining functional mitral regurgitation beyond left ventricular geometry after narrow qrs anterior myocardial infarction: A real time three-dimensional echocardiography study. Echocardiography. 2011;28:665-675

39. Kanzaki H, Bazaz R, Schwartzman D, Dohi K, Sade LE, Gorcsan J, 3rd. A mechanism for immediate reduction in mitral regurgitation after cardiac resynchronization therapy: Insights from mechanical activation strain mapping. Journal of the American College of Cardiology. 2004;44:1619-1625

40. Appleton CP, Basnight MA, Gonzalez MS. Diastolic mitral regurgitation with atrioventricular conduction abnormalities: Relation of mitral flow velocity to transmitral pressure gradients in conscious dogs. Journal of the American College of Cardiology. 1991;18:843-849

41. Agricola E, Oppizzi M, Galderisi M, Pisani M, Meris A, Pappone C, Margonato A. Role of regional mechanical dyssynchrony as a determinant of functional mitral regurgitation in patients with left ventricular systolic dysfunction. Heart. 2006;92:1390-1395

42. Breithardt OA, Sinha AM, Schwammenthal E, Bidaoui N, Markus KU, Franke A, Stellbrink C. Acute effects of cardiac resynchronization therapy on functional mitral regurgitation in advanced systolic heart failure. Journal of the American College of Cardiology. 2003;41:765-770

43. Lancellotti P, Melon P, Sakalihasan N, Waleffe A, Dubois C, Bertholet M, Pierard LA. Effect of cardiac resynchronization therapy on functional mitral regurgitation in heart failure. The American journal of cardiology. 2004;94:1462-1465

44. Van Garsse L, Gelsomino S, Cheriex E, Luca F, Rao CM, Parise O, Gensini GF, Maessen J. Tethering symmetry reflects advanced left ventricular mechanical dyssynchrony in patients with ischemic mitral regurgitation undergoing restrictive mitral valve repair. The Annals of thoracic surgery. 2012;94:14181428

45. Van Garsse L, Gelsomino S, Luca F, Parise O, Lorusso R, Cheriex E, Caciolli S, Vizzardi E, Rao CM, Carella $\mathrm{R}$, Gensini GF, Maessen J. Left ventricular dyssynchrony is associated with recurrence of ischemic mitral regurgitation after restrictive annuloplasty. International journal of cardiology. 2012

46. Van Garsse L, Gelsomino S, Parise O, Luca F, Cheriex E, Lorusso R, Vizzardi E, Rao CM, Gensini GF, Maessen J. Systolic papillary muscle dyssynchrony predicts recurrence of mitral regurgitation in patients with ischemic cardiomyopathy (icm) undergoing mitral valve repair. Echocardiography. 2012 
47. Tigen K, Karaahmet T, Dundar C, Guler A, Cevik C, Basaran O, Kirma C, Basaran Y. The importance of papillary muscle dyssynchrony in predicting the severity of functional mitral regurgitation in patients with non-ischaemic dilated cardiomyopathy: A two-dimensional speckle-tracking echocardiography study. European journal of echocardiography: the journal of the Working Group on Echocardiography of the European Society of Cardiology. 2010;11:671-676

48. Bleeker GB, Holman ER, Steendijk P, Boersma E, van der Wall EE, Schalij MJ, Bax JJ. Cardiac resynchronization therapy in patients with a narrow qrs complex. Journal of the American College of Cardiology. 2006;48:2243-2250

49. Suffoletto MS, Dohi K, Cannesson M, Saba S, Gorcsan J, 3rd. Novel speckle-tracking radial strain from routine black-and-white echocardiographic images to quantify dyssynchrony and predict response to cardiac resynchronization therapy. Circulation. 2006;113:960-968

50. Ypenburg C, Lancellotti P, Tops LF, Bleeker GB, Holman ER, Pierard LA, Schalij MJ, Bax JJ. Acute effects of initiation and withdrawal of cardiac resynchronization therapy on papillary muscle dyssynchrony and mitral regurgitation. Journal of the American College of Cardiology. 2007;50:2071-2077

51. Ypenburg C, Lancellotti P, Tops LF, Boersma E, Bleeker GB, Holman ER, Thomas JD, Schalij MJ, Pierard LA, Bax JJ. Mechanism of improvement in mitral regurgitation after cardiac resynchronization therapy. European heart journal. 2008;29:757-765

52. Messas E, Guerrero JL, Handschumacher MD, Chow CM, Sullivan S, Schwammenthal E, Levine RA. Paradoxic decrease in ischemic mitral regurgitation with papillary muscle dysfunction: Insights from three-dimensional and contrast echocardiography with strain rate measurement. Circulation. 2001;104:1952-1957

53. Uemura T, Otsuji Y, Nakashiki K, Yoshifuku S, Maki Y, Yu B, Mizukami N, Kuwahara E, Hamasaki S, Biro S, Kisanuki A, Minagoe S, Levine RA, Tei C. Papillary muscle dysfunction attenuates ischemic mitral regurgitation in patients with localized basal inferior left ventricular remodeling: Insights from tissue doppler strain imaging. Journal of the American College of Cardiology. 2005;46:113-119

54. Cerqueira MD, Weissman NJ, Dilsizian V, Jacobs AK, Kaul S, Laskey WK, Pennell DJ, Rumberger JA, Ryan T, Verani MS. Standardized myocardial segmentation and nomenclature for tomographic imaging of the heart: A statement for healthcare professionals from the cardiac imaging committee of the council on clinical cardiology of the american heart association. Circulation. 2002;105:539-542

55. Cazeau S, Leclercq C, Lavergne T, Walker S, Varma C, Linde C, Garrigue S, Kappenberger L, Haywood GA, Santini M, Bailleul C, Daubert JC. Effects of multisite biventricular pacing in patients with heart failure and intraventricular conduction delay. The New England journal of medicine. 2001;344:873-880

56. Abraham WT, Fisher WG, Smith AL, Delurgio DB, Leon AR, Loh E, Kocovic DZ, Packer M, Clavell AL, Hayes DL, Ellestad M, Trupp RJ, Underwood J, Pickering F, Truex C, McAtee P, Messenger J. Cardiac resynchronization in chronic heart failure. The New England journal of medicine. 2002;346:1845-1853

57. Bristow MR, Saxon LA, Boehmer J, Krueger S, Kass DA, De Marco T, Carson P, DiCarlo L, DeMets D, White BG, DeVries DW, Feldman AM. Cardiac-resynchronization therapy with or without an implantable defibrillator in advanced chronic heart failure. The New England journal of medicine. 2004;350:2140-2150

58. Cleland JG, Daubert JC, Erdmann E, Freemantle N, Gras D, Kappenberger L, Tavazzi L. The effect of cardiac resynchronization on morbidity and mortality in heart failure. The New England journal of medicine. 2005;352:1539-1549

59. Strickberger SA, Conti J, Daoud EG, Havranek E, Mehra MR, Pina IL, Young J. Patient selection for cardiac resynchronization therapy: From the council on clinical cardiology subcommittee on electrocardiography and arrhythmias and the quality of care and outcomes research interdisciplinary working group, in collaboration with the heart rhythm society. Circulation. 2005;111:2146-2150

60. Bleeker GB, Schalij MJ, Molhoek SG, Holman ER, Verwey HF, Steendijk P, van der Wall EE, Bax JJ. Frequency of left ventricular dyssynchrony in patients with heart failure and a narrow qrs complex. The American journal of cardiology. 2005;95:140-142 
61. Bleeker GB, Schalij MJ, Molhoek SG, Verwey HF, Holman ER, Boersma E, Steendijk P, Van Der Wall EE, Bax JJ. Relationship between qrs duration and left ventricular dyssynchrony in patients with end-stage heart failure. Journal of cardiovascular electrophysiology. 2004;15:544-549

62. Rouleau F, Merheb M, Geffroy S, Berthelot J, Chaleil D, Dupuis JM, Victor J, Geslin P. Echocardiographic assessment of the interventricular delay of activation and correlation to the qrs width in dilated cardiomyopathy. Pacing and clinical electrophysiology: PACE. 2001;24:1500-1506

63. $\mathrm{Yu}$ CM, Lin $\mathrm{H}$, Zhang $\mathrm{Q}$, Sanderson JE. High prevalence of left ventricular systolic and diastolic asynchrony in patients with congestive heart failure and normal qrs duration. Heart. 2003;89:54-60

64. Shaw RM, Rudy Y. Electrophysiologic effects of acute myocardial ischemia. A mechanistic investigation of action potential conduction and conduction failure. Circulation research. 1997;80:124-138

65. Forrester JS, Wyatt HL, Da Luz PL, Tyberg JV, Diamond GA, Swan HJ. Functional significance of regional ischemic contraction abnormalities. Circulation. 1976;54:64-70

66. Celikyurt U, Vural A, Sahin T, Kilic T, Agacdiken A, Ural D. Relationship between left ventricular dyssynchrony and reverse remodeling after cardiac resynchronization therapy. Clinical cardiology. 2011;34:645-648

67. Agricola E, Oppizzi M, Maisano F, De Bonis M, Schinkel AF, Torracca L, Margonato A, Melisurgo G, Alfieri O. Echocardiographic classification of chronic ischemic mitral regurgitation caused by restricted motion according to tethering pattern. European journal of echocardiography: the journal of the Working Group on Echocardiography of the European Society of Cardiology. 2004;5:326-334

68. Otsuji Y, Handschumacher MD, Schwammenthal E, Jiang L, Song JK, Guerrero JL, Vlahakes GJ, Levine RA. Insights from three-dimensional echocardiography into the mechanism of functional mitral regurgitation: Direct in vivo demonstration of altered leaflet tethering geometry. Circulation. 1997;96:1999-2008

69. Yiu SF, Enriquez-Sarano M, Tribouilloy C, Seward JB, Tajik AJ. Determinants of the degree of functional mitral regurgitation in patients with systolic left ventricular dysfunction: A quantitative clinical study. Circulation. 2000;102:1400-1406

70. Voci P, Bilotta F, Caretta Q, Mercanti C, Marino B. Papillary muscle perfusion pattern. A hypothesis for ischemic papillary muscle dysfunction. Circulation. 1995;91:1714-1718

71. Kumanohoso T, Otsuji Y, Yoshifuku S, Matsukida K, Koriyama C, Kisanuki A, Minagoe S, Levine RA, Tei C. Mechanism of higher incidence of ischemic mitral regurgitation in patients with inferior myocardial infarction: Quantitative analysis of left ventricular and mitral valve geometry in 103 patients with prior myocardial infarction. The Journal of thoracic and cardiovascular surgery. 2003;125:135-143

72. D'Andrea A, Caso P, Romano S, Scarafile R, Riegler L, Salerno G, Limongelli G, Di Salvo G, Calabro P, Del Viscovo L, Romano G, Maiello C, Santangelo L, Severino S, Cuomo S, Cotrufo M, Calabro R. Different effects of cardiac resynchronization therapy on left atrial function in patients with either idiopathic or ischaemic dilated cardiomyopathy: A two-dimensional speckle strain study. European heart journal. 2007; $28: 2738-2748$

73. Cianciulli TF, Saccheri MC, Lax JA, Bermann AM, Ferreiro DE. Two-dimensional speckle tracking echocardiography for the assessment of atrial function. World journal of cardiology. 2010;2:163-170

74. Van Garsse L, Gelsomino S, Luca F, Parise O, Cheriex E, Rao CM, Gensini GF, Maessen JG. Left atrial strain and strain rate before and following restrictive annuloplasty for ischaemic mitral regurgitation evaluated by two-dimensional speckle tracking echocardiography. European heart journal cardiovascular Imaging. 2012

75. Cox JL, Chiasson DA, Gotlieb Al. Stranger in a strange land: The pathogenesis of saphenous vein graft stenosis with emphasis on structural and functional differences between veins and arteries. Progress in cardiovascular diseases. 1991;34:45-68

76. Chazova I, Robbins I, Loyd J, Newman J, Tapson V, Zhdaov V, Meyrick B. Venous and arterial changes in pulmonary veno-occlusive disease, mitral stenosis and fibrosing mediastinitis. The European respiratory journal: official journal of the European Society for Clinical Respiratory Physiology. 2000;15:116-122 
77. Kuppahally SS, Akoum N, Burgon NS, Badger TJ, Kholmovski EG, Vijayakumar S, Rao SN, Blauer J, Fish EN, Dibella EV, Macleod RS, McGann C, Litwin SE, Marrouche NF. Left atrial strain and strain rate in patients with paroxysmal and persistent atrial fibrillation: Relationship to left atrial structural remodeling detected by delayed-enhancement mri. Circulation. Cardiovascular imaging. 2010;3:231239

78. Kokubu N, Yuda S, Tsuchihashi K, Hashimoto A, Nakata T, Miura T, Ura N, Nagao K, Tsuzuki M, Wakabayashi $C$, Shimamoto K. Noninvasive assessment of left atrial function by strain rate imaging in patients with hypertension: A possible beneficial effect of renin-angiotensin system inhibition on left atrial function. Hypertension research: official journal of the Japanese Society of Hypertension. 2007;30:13-21

79. Spencer KT, Mor-Avi V, Gorcsan J, 3rd, DeMaria AN, Kimball TR, Monaghan MJ, Perez JE, Weinert L, Bednarz J, Edelman K, Kwan OL, Glascock B, Hancock J, Baumann C, Lang RM. Effects of aging on left atrial reservoir, conduit, and booster pump function: A multi-institution acoustic quantification study. Heart. 2001;85:272-277

80. Mosseri M, Yarom R, Gotsman MS, Hasin Y. Histologic evidence for small-vessel coronary artery disease in patients with angina pectoris and patent large coronary arteries. Circulation. 1986;74:964-972

81. Diegeler A, Spyrantis N, Matin M, Falk V, Hambrecht R, Autschbach R, Mohr FW, Schuler G. The revival of surgical treatment for isolated proximal high grade lad lesions by minimally invasive coronary artery bypass grafting. European journal of cardio-thoracic surgery: official journal of the European Association for Cardio-thoracic Surgery. 2000;17:501-504

82. Johnson BD, Shaw LJ, Buchthal SD, Bairey Merz CN, Kim HW, Scott KN, Doyle M, Olson MB, Pepine CJ, den Hollander J, Sharaf B, Rogers WJ, Mankad S, Forder JR, Kelsey SF, Pohost GM. Prognosis in women with myocardial ischemia in the absence of obstructive coronary disease: Results from the national institutes of health-national heart, lung, and blood institute-sponsored women's ischemia syndrome evaluation (wise). Circulation. 2004;109:2993-2999

83. Pepine CJ, Handberg EM, Cooper-DeHoff RM, Marks RG, Kowey P, Messerli FH, Mancia G, Cangiano JL, Garcia-Barreto D, Keltai M, Erdine S, Bristol HA, Kolb HR, Bakris GL, Cohen JD, Parmley WW. A calcium antagonist vs a non-calcium antagonist hypertension treatment strategy for patients with coronary artery disease. The international verapamil-trandolapril study (invest): A randomized controlled trial. JAMA: the journal of the American Medical Association. 2003;290:2805-2816

84. von Mering GO, Arant CB, Wessel TR, McGorray SP, Bairey Merz CN, Sharaf BL, Smith KM, Olson MB, Johnson BD, Sopko G, Handberg E, Pepine CJ, Kerensky RA. Abnormal coronary vasomotion as a prognostic indicator of cardiovascular events in women: Results from the national heart, lung, and blood institute-sponsored women's ischemia syndrome evaluation (wise). Circulation. 2004;109:722725

85. Prevalence of unrecognized silent myocardial ischemia and its association with atherosclerotic risk factors in noninsulin-dependent diabetes mellitus. Milan study on atherosclerosis and diabetes (misad) group. The American journal of cardiology. 1997;79:134-139

86. Stein B, Weintraub WS, Gebhart SP, Cohen-Bernstein CL, Grosswald R, Liberman HA, Douglas JS, Jr., Morris DC, King SB, 3rd. Influence of diabetes mellitus on early and late outcome after percutaneous transluminal coronary angioplasty. Circulation. 1995;91:979-989

87. Carneiro AV. Coronary heart disease in diabetes mellitus: Risk factors and epidemiology. Revista portuguesa de cardiologia: orgao oficial da Sociedade Portuguesa de Cardiologia = Portuguese journal of cardiology: an official journal of the Portuguese Society of Cardiology. 2004;23:1359-1366

88. Ambrose JA, Tannenbaum MA, Alexopoulos D, Hjemdahl-Monsen CE, Leavy J, Weiss M, Borrico S, Gorlin R, Fuster V. Angiographic progression of coronary artery disease and the development of myocardial infarction. Journal of the American College of Cardiology. 1988;12:56-62

89. Murry CE, Jennings RB, Reimer KA. Preconditioning with ischemia: A delay of lethal cell injury in ischemic myocardium. Circulation. 1986;74:1124-1136 
90. Bax JJ, Schinkel AF, Boersma E, Elhendy A, Rizzello V, Maat A, Roelandt JR, van der Wall EE, Poldermans D. Extensive left ventricular remodeling does not allow viable myocardium to improve in left ventricular ejection fraction after revascularization and is associated with worse long-term prognosis. Circulation. 2004;110:II18-22

91. Alfieri O, Maisano F, De Bonis M, Stefano PL, Torracca L, Oppizzi M, La Canna G. The double-orifice technique in mitral valve repair: A simple solution for complex problems. The Journal of thoracic and cardiovascular surgery. 2001;122:674-681

92. Messas E, Pouzet B, Touchot B, Guerrero JL, Vlahakes GJ, Desnos M, Menasche P, Hagege A, Levine RA. Efficacy of chordal cutting to relieve chronic persistent ischemic mitral regurgitation. Circulation. 2003;108 Suppl 1:II11-II115

93. Kron IL, Green GR, Cope JT. Surgical relocation of the posterior papillary muscle in chronic ischemic mitral regurgitation. The Annals of thoracic surgery. 2002;74:600-601

94. Livi U, Alfieri O, Vitali E, Russo C, Frigerio M, Tursi V, Albanese MC, De Bonis M, Fragasso G, FrancoCereceda A, Forssell G, Rorke R, Kubo SH. One-year clinical experience with the acorn corcap cardiac support device: Results of a limited market release safety study in italy and sweden. Italian heart journal: official journal of the Italian Federation of Cardiology. 2005;6:59-65

95. de Bakker JM, van Capelle FJ, Janse MJ, Tasseron S, Vermeulen JT, de Jonge N, Lahpor JR. Slow conduction in the infarcted human heart. 'Zigzag' course of activation. Circulation. 1993;88:915-926

96. Valencik ML, Zhang D, Punske B, Hu P, McDonald JA, Litwin SE. Integrin activation in the heart: A link between electrical and contractile dysfunction? Circulation research. 2006;99:1403-1410 
Chapter 10

Summary

Samenvatting (Summary in Dutch)

Dankwoord

Curriculum vitae

List of publications

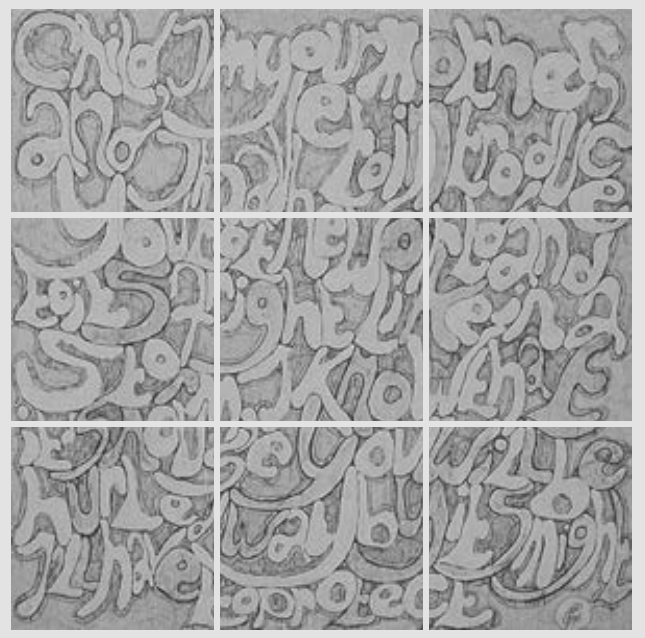





\section{Summary}

In patients with chronic ischemic mitral regurgitation (CIMR), mitral leaflet closure is hindered by traction on the leaflets (leaflet tethering) because of ischemic ventricular disease. Therefore CIMR has been treated by restrictive annuloplasty (RA), combined with coronary bypass grafting (CABG). However, despite the enthusiasm for low perioperative mortality rate, high initial surgical success rate (no residual MR) and the positive effects on survival and functional symptomatic status, several studies have clearly demonstrated that RA is subject to variable rates of recurrence of MR. Mechanisms for recurrence have been described. Progressive left ventricular adverse remodeling (LVR), local or global, seems to be the crowbar to distort the balance of valve closure, causing recurrence of MR. However, recent studies have confirmed that some patients with CIMR may benefit from RA. To select patients who will benefit from RA a search for preoperative predictors of recurrent MR is needed.

In the first chapter of this thesis, the definition of CIMR, the pathophysiology, prevalence and outcome of patients without valve treatment are clarified. Historical notes and studies are reported and the contribution of individual components of the mitral valve complex to CIMR is extensively described to understand the mechanism of ischemic mitral insufficiency. The echocardiographic evaluation of these patients concentrates on mitral valve function and geometry as well as on left ventricular and papillary muscular characteristics. Functional evaluation by strain and strain rate is explained. Finally, the outcome of treatment by CABG and RA in CIMR patients is reported and mechanisms of recurrent MR are described. The research in the following chapters is based on a search of the predictors of recurrent MR, trying to select the patients that will benefit from CABG and RA.

Recently, some reports have indicated the preoperative geometrical configuration of the posterior mitral leaflet ( $\mathrm{PML}$ tethering angle $>45^{\circ}$ ) as a predictor of recurrent $\mathrm{MR}$. However, in Chapter $\mathbf{2}$ we investigate the importance of the anterior mitral leaflet $(A M L)$ tethering angle in predicting recurrence of $M R$ and whether the effect of $A M L$ tethering is secondary to modifications in left ventricular size and geometry. In a large multicenter study (435 patients), patients were divided by the baseline AML tethering angle. The predictive value of AML tethering was confirmed. Moreover, compared with patients with $\mathrm{PML}$ tethering $\geq 45^{\circ}$, those with moderate-severe AML tethering had 1.7 times higher odds of MR recurrence. The increase in risk associated with severe AML tethering was $>3.7$-fold. Furthermore, we did not find any interaction between AML 
tethering and other variables of impaired LV function and LVR (geometrical alterations) demonstrating that $A M L$ tethering is a primary independent predictor of $M R$, and its effect is not secondary to left ventricular function and geometry.

In Chapter 3 we investigate the impact of preoperative AML tethering on the recurrence of $M R$ and left ventricular reverse remodeling (LVRR), defined as a reduction in end-systolic volume index $>15 \%$ and left ventricular geometrical reverse remodeling (LVGRR), defined as a reduction in systolic sphericity index to a normal value of $<0.72$ in patients with altered baseline geometry. In this multicenter study, 362 patients were divided into two groups by baseline AML tethering. At multivariable regression analysis an $A M L$ tethering angle $\geq 39.5^{\circ}$ was a strong predictor of $M R$ recurrence, lack of LVRR and lack of LVGRR. In contrast, the preoperative PML tethering angle did not show a significant difference in either group.

LV dyssynchrony contributes to functional mitral regurgitation (MR) since the uncoordinated mechanical contraction of papillary muscle insertion sites leads to a decreased MV closing force, which causes MR. In Chapter 4 we investigate whether dyssynchrony of the papillary muscles (DYS-PAP) is a predictor of recurrence of MR. These measurements were based on 2D-speckle-tracking echocardiography of the papillary muscles and we found that DYS-PAP $\geq 58 \mathrm{~ms}$ was a strong predictor of recurrent MR. We showed that DYS-PAP represents a reliable tool to identify patients with CIMR who can benefit from CABG and RA.

DYS-PAP $\geq 58$ ms was evaluated as a predictor of recurrent MR in a specific population of patients with ischemic dilated cardiomyopathy MR (ejection fraction < 35\%) who underwent CABG and RA. In Chapter 5 we confirm that DYS-PAP is a strong predictor of $M R$ recurrence with a maximal sensitivity and high specificity.

The relationship between the mitral leaflet tethering pattern and DYS-PAP is investigated in Chapter 6. The preoperative symmetric tethering pattern is significantly correlated with preoperative DYS-PAP. We found that in symmetric tethering patients DYS-PAP deteriorated during follow up (postoperatively), while in the asymmetric tethering patients DYS-PAP improved significantly. We concluded that the symmetric pattern is a strong predictor of postoperative DYS-PAP. The tethering pattern seems to be an easy and immediate tool to identify patients with advanced DYS-PAP who are unlikely to benefit from CABG and RA.

By searching for predictors of recurrent $M R$, apparently the population with recurrence has more severe ventricular dysfunction at baseline, which can obviously not be compensated by CABG and RA compared to patients that have no recurrence. In Chapter 7 we measure the strain (longitudinal, radial and circumferential) of 16 LV 
segments in patients with a symmetric and asymmetric tethering pattern at baseline and follow up (after CABG and RA). We demonstrate that patients with a baseline symmetric tethering pattern had impaired strain values in all LV segments at baseline, which were not reverted by surgery. In contrast, asymmetric patients showed only localized impaired strain values, which significantly improved at follow up.

Identification of the CIMR population that will have recurrent MR after CABG and RA was investigated in the previous chapters at the level of mitral valve geometrics, papillary muscle dyssynchrony and LV function. Since mitral valve closure is also dependent on the transmitral gradient, left atrial (LA) function is evaluated in Chapter 8 by LA strain and strain rate, before and after CABG and RA. Patients with recurrent MR observed an irreversible impairment of the LA reservoir phase due to reduced compliance. In these patients also the conduit phase and active contractile phase did not improve after the operation, which was probably attributable to high LV filling pressure. Patients without recurrent MR had enhanced LA volume and strain values at baseline, which reverted to normal measurements at follow up.

In Chapter 9 an overview of the thesis and further perspectives are discussed. Looking at the leaflet configuration, it is explained why the angle of AML (and not PML) is a predictor of recurrent MR. Moreover, differentiation of the basal and distal AML angle is discussed. The relation between the AML angle and LV remodeling (global and local) is explained and when looking at local remodeling, defined by PM displacement, it seems that ALPM displacement determines the AML configuration.

Although there is no relation between PM displacement and function of the PMs (fiber shortening), it seems that function of the LV wall, adjacent to the PMs and PM displacement, varies in a similar way.

In previous chapters, papillary muscle dyssynchrony (DYS-PAP) was shown to be predictor of recurrent MR. However, it is important to differentiate LV dyssynchrony from DYS-PAP and to differentiate mechanical dyssynchrony from electrical dyssynchrony.

A symmetric leaflet tethering pattern seems to be a strong predictor of papillary muscle dyssynchrony and reflects patients with generalized ischemic heart disease. In contrast, an asymmetric tethering pattern reflects patients with localized ischemic disease.

To select patients who will benefit from RA, LA function has to be evaluated since LA function denotes diastolic LV (dys)function. LA volume and reservoir are reversible as long as structural changes of the LA wall are absent. Dysfunction of the LA conduit and contraction depicts a population with irreversible LV diastolic dysfunction with lack of reversed remodeling, even after RA and CABG. 
We conclude that RA can only be effectively employed in selected CIMR patients. RA is indicated in CIMR patients with an echocardiographic asymmetric mitral valve tethering pattern, with an $\mathrm{AML}$ tethering angle $<39.5^{\circ}, \mathrm{SI}_{\mathrm{S}}<0.7$ and in the absence of severe DYS-PAP (<58 ms), and advanced diastolic LV dysfunction (reduced LA function). These echocardiographic variables should be incorporated in the preoperative assessment of patients with CIMR. 


\section{Samenvatting (Summary in Dutch)}

Bij patiënten met chronische ischemische mitralisklep insufficiëntie (CIMR), wordt de sluiting van de mitralisklep gehinderd door tractie op de twee klepbladen als gevolg van een ischemische aandoening van de hartspier. Daarom heeft de standaard behandeling voor patiënten met CIMR zich de laatste 20 jaren toegespitst op zowel het ischemisch hartlijden, doormiddel van coronaire bypass chirurgie (CABG), als op de mitralisklep insufficiëntie (MR), met behulp van een restrictieve ring (RA) die men op de mitralisklep annulus hecht. Echter, ondanks het initiële enthousiasme voor deze ingreep, gezien het lage perioperatieve sterftecijfer, de goede initiële chirurgische resultaten (geen rest-lekkage van de mitralisklep na de ingreep) en de gunstige symptomatische uitkomst, hebben verschillende recente studies aangetoond dat meer dan $30 \%$ van deze patiënten na 3 jaar terug matig-tot-ernstige $M R(M R \geq 2+$ ) vertonen . Het mechanisme voor dit recidief berust op een progressieve remodeling van de linker ventrikel (LVR), die gelokaliseerd of globaal kan voorkomen. Toch zijn er ook recente studies die bevestigen dat sommige CIMR patiënten wel baat hebben bij de gecombineerde behandeling van CABG met een restrictieve ring. Een goede patiënten selectie is dus cruciaal. Daarom wordt getracht via dit onderzoek om echocardiografische parameters te identificeren die het recidief op MR kunnen voorspellen.

In het eerste hoofdstuk wordt de definitie, de pathofysiologie en prevalentie van CIMR verduidelijkt. De normale, maar complexe samenstelling van het mitralisklep-apparaat, bestaande uit 5 componenten wordt beschreven en de bijdrage van deze componenten tot CIMR wordt ook via historische studies toegelicht. De echocardiografische evaluatie van deze patiënten concentreert zich niet enkel op de mitralisklep functie en de geometrische configuratie van de klep, maar ook op de functie en geometrie van de linker ventrikel (LV) en de papillair spieren (PM). Voor de functionele evaluatie van spierweefsel, aan de hand van strain metingen, wordt gebruik gemaakt van 2Dspeckle-tracking echocardiografie (2D-STE), een software- analyse die in dit hoofdstuk wordt besproken.

De slechte uitkomst bij CIMR patiënten ( $M R \geq 2+$ ), waarbij enkel een CABG werd verricht, benadrukt het belang van een additionele behandeling voor de mitralisklep insufficiëntie. In dit hoofdstuk worden de uiteenlopende resultaten na CABG en restrictieve annuloplastie aangehaald, wat meteen ook de reden is van ons onderzoek naar voorspellende parameters om patiënten te kunnen selecteren die wel baat hebben bij deze operatieve behandeling. 
Een aantal recente studies hebben de geometrische configuratie van het achterste mitralisklepblad (PML) aangeduid als predictor voor recidief $M R$ na CABG en RA. Een mid-systolische $\mathrm{PML}$ tractie hoek $\geq 45^{\circ}$ zou recidief MR voorspellen. Echter, in hoofdstuk 2 onderzoeken we door middel van een grote multicenter studie (435 CIMR patienten) het belang van de tractie hoek van het voorste klepblad (AML) als predictor voor recidief MR. Bovendien onderzoeken we of het effect van deze hoek niet secundair is aan andere parameters waarvan in eerder onderzoek werd aangetoond dat ze geassocieerd zijn met recidief MR, zoals verminderde LV functie, LV dilatatie en de geometrische configuratie van LV, neigend naar een bolvorm (sfericiteitsindex). De voorspellende waarde van AML-tractie wordt in de studie bevestigd. Daarenboven heeft de studie aangetoond dat patiënten met een matig-tot-ernstige $\left(36.9^{\circ} \leq \mathrm{AML}<\right.$ $40.1^{\circ}$ ) en ernstige $\left(A M L>40.1^{\circ}\right.$ ) tractie op het $A M L$ een 1.7, respectievelijk 3.7 keer grotere kans hebben op recidief $M R$, in vergelijking met de patiënten, geselecteerd op basis van $\mathrm{PML} \geq 45^{\circ}$. Er werd bovendien geen enkele interactie gevonden tussen de AML tractie en andere variabelen die duiden op LV functie, volume en geometrie, waardoor we hebben aangetoond dat de AML tractie een primaire, onafhankelijke voorspellende parameter is voor recidief MR.

In hoofdstuk 3 wordt de relatie onderzocht tussen de predictor voor recidief MR (AML tractie hoek) en parameters die verband houden met de remodeling van de LV, gezien in eerdere studies werd aangetoond dat progressieve LV remodeling het mechanisme is voor recidief MR. Hierbij werd niet alleen de reverse remodeling van de LV (LVRR) onderzocht, gedefinieerd als een afname van de eind-systolische volume-index $>15 \%$, maar ook de geometrische reverse remodeling (LVGRR) van de LV, welke gedefinieerd werd als een daling van de systolische sfericiteitsindex naar een waarde $<0.72$ bij patienten waarbij deze waarde preoperatief verhoogd was. In deze multicenter studie (362 patiënten) werd aangetoond dat de preoperatieve AML tractie hoek $\geq 39.5^{\circ}$ een sterke predictor was voor recidief MR, maar ook predictor voor gebrek aan LVRR en LVGRR.

LV dyssynchronie draagt bij aan functionele MR omdat de ongecoördineerde contractie van de papillair spieren resulteert in een verminderde kracht om de mitralisklep bladen te sluiten. In hoofdstuk 4 onderzoeken we of de dyssynchronie van de papillair spieren (DYS-PAP) een predictor is voor recidief MR. De metingen voor DYS-PAP zijn gebaseerd op strain metingen doormiddel van 2D speckle-tracking echocardiografie op het niveau van de papillair spieren zelf. Uit de studie blijkt dat DYS-PAP $\geq 58 \mathrm{~ms}$ een sterke predictor is voor recidief MR in CIMR patiënten. Bovendien neemt de DYS-PAP progressief toe bij de patiënten met recidief $M R$, terwijl deze significant daalt bij patienten die geen MR vertonen tijdens follow up. 
In hoofdstuk 5 wordt het belang van de DYS-PAP $\geq 58 \mathrm{~ms}$ als predictor voor recidief $M R$ bevestigd in een meer specifieke patiënten populatie, met name de patiënten met een ischemische gedilateerde cardiomyopathie (LV ejectiefractie < 35\%). Ernstige dyssynchronie blijkt de vicieuze cirkel van LV remodeling verder te ondersteunen, terwijl een afname van dyssynchronie resulteert in een verbetering van LV functie en ontwikkeling van LVRR. Ernstige preoperatieve DYS-PAP is een indicator voor gevorderde myocard schade in patiënten met ischemisch gedilateerde cardiomyopathie, waarbij irreversibele progressie van remodeling te verwachten is, ondanks CABG en restrictieve ring annuloplastie.

In hoofdstuk 6 wordt de relatie tussen het tractiepatroon van de mitralisklepblaadjes en de dyssynchronie van de papillair spieren (PM) onderzocht. Uit de studie in dit hoofdstuk blijkt dat een symmetrisch tractiepatroon gecorreleerd is met ernstige preoperatieve DYS-PAP, terwijl een asymmetrisch tractiepatroon gepaard gaat met significant lagere DYS-PAP waarden. In het postoperatieve beloop neemt de DYS-PAP progressief toe bij patiënten met een symmetrisch preoperatief tractiepatroon, terwijl deze bij patiënten met een asymmetrische patroon aanzienlijk afneemt. We concluderen dat een symmetrisch tractiepatroon van de mitralisklepblaadjes een duidelijke predictor is voor postoperatieve DYS-PAP. Bovendien is het tractiepatroon een eenvoudig te meten parameter om CIMR patiënten met gevorderde DYS-PAP te identificeren, die vermoedelijk geen baat hebben bij CABG met restrictieve annuloplastie.

CIMR patiënten met recidief MR vertonen een meer diffuse en meer ernstige aantasting van de LV functie, die blijkbaar niet recupereert na CABG en restrictieve annuloplastie, in vergelijking met patiënten bij wie geen recidief MR optreedt. In hoofdstuk 7 wordt de segmentale LV functie door middel van strain (longitudinaal, radiair en circumferentieel) gemeten bij CIMR patiënten met een symmetrisch en asymmetrisch tractiepatroon van de mitralisklep, zowel preoperatief als tijdens de follow up. Deze studie toont aan dat patiënten met een preoperatief symmetrisch tractiepatroon een verminderde functie vertonen in alle LV segmenten, dewelke zich postoperatief niet herstellen. Anderzijds, blijkt dat de functie afwijkingen bij patiënten met een asymmetrisch patroon gelokaliseerd voorkomen en dat deze recupereren tijdens follow up.

De identificatie van CIMR patiënten bij wie na CABG met restrictieve annuloplastie recidief $M R$ te verwachten is, werd in voorgaande hoofdstukken onderzocht op het niveau van de mitralisklep geometrie, de papillair spier dyssynchronie en de LV functie. Echter, een adequate sluiting van de mitralisklep is ook afhankelijk van de transmitrale gradiënt. Daarom wordt in hoofdstuk 8 de linker atrium (LA) functie onderzocht aan de hand van LA strain en strain rate bij CIMR patiënten, voor en na CABG met restrictieve annuloplastie. Uit deze studie blijkt dat patiënten met een recidief MR een afname tonen van de LA reservoir functie. Deze afname blijft postoperatief irreversibel bestaan 
en is vermoedelijk te wijten aan de gereduceerde compliantie van de LA wand. Bij deze patiënten wordt ook geen recuperatie aangetoond van de conduit functie en de actieve atriale contractie, vermoedelijk ten gevolge van de hoge LV vullingsdrukken bij recidief MR. Patiënten zonder recidief MR vertonen preoperatief zelfs supra-normale waarden voor LA volume en functie, dewelke normaliseren postoperatief.

In hoofdstuk 9 wordt het overzicht van de thesis geschetst en worden de verschillende gezichtspunten getoetst aan de hand van de huidige literatuur. De afweging van geometrische configuratie tussen AML en PML als predictor voor recidief MR wordt toegelicht, alsook het onderscheid tussen de basale en distale tractiehoek van het AML. De relatie tussen de AML hoek en de LV remodeling (zowel gelokaliseerd als globaal) wordt aangehaald. We concluderen dat het blijkbaar de dislocatie is van de anterolaterale PM die bepalend is voor de AML configuratie. Alhoewel er geen relatie is tussen de PM dislocatie en de functie van de PMs (de effectieve verkorting van de spiervezels), lijkt het er wel op dat de functie van de LV spier, die de PM ondersteunt, en de dislocatie van de PM in dezelfde mate veranderen.

In voorgaande hoofdstukken werd aangetoond dat DYS-PAP predictor is voor recidief MR. Het is echter belangrijk om een onderscheid te maken tussen LV dyssynchronie en papillair spier dyssynchronie en om een onderscheid te maken tussen mechanische en elektrische dyssynchronie.

De relatie tussen het symmetrisch tractiepatroon van de mitralisklepblaadjes als predictor voor DYS-PAP wordt aangehaald en blijkbaar is dit een reflectie van de patiënten met gegeneraliseerd, irreversibel ischemisch hartlijden. De patiënten met een asymmetrisch tractiepatroon zijn blijkbaar een weergave van de patiëntengroep met gelokaliseerd, reversibel hartlijden.

Het belang van de LA functie voor de selectie van CIMR patiënten die baat hebben bij CABG en restrictieve annuloplastie, wordt toegelicht. Uit de literatuur is af te leiden dat zowel LA volume als reservoirfunctie vermoedelijk reversibel zijn zolang er geen structurele veranderingen in de LA wand zijn aangetoond. Dysfunctie van LA conduit en contractie toont dus een irreversibele LV diastolische dysfunctie zonder LVRR na CABG en restrictieve annuloplastie.

We besluiten dat restrictieve annuloplastie alleen effectief kan aangewend worden in een geselecteerde groep van patiënten met CIMR. De voorspellende parameters voor recidief MR zijn: een symmetrisch tractiepatroon van de mitralisklepblaadjes, een tractie hoek van het $A M L \geq 39.5^{\circ}$, een systolische sfericiteitsindex $\geq 0.7$ en een ernstige DYS-PAP ( $\geq 58 \mathrm{~ms}$ ). Een gereduceerde LA functie is indicatief voor een gevorderde diastolische LV dysfunctie. In aanwezigheid van deze parameters blijkt dat 
CIMR patiënten geen baat hebben bij CABG met restrictieve annuloplastie. Deze echocardiografische parameters zouden moeten opgenomen worden in het preoperatieve bilan van patiënten met CIMR. 



\section{Dankwoord}

Dit proefschrift is tot stand gekomen door de bijdrage van vele mensen en is het resultaat van teamwork. Ik wil graag van deze gelegenheid gebruik maken om hen te bedanken voor hun steun, inzet en vertrouwen.

Mijn dank gaat uit naar mijn promotor, Prof. Dr. J. Maessen. Beste Jos, ik wil je danken voor je steun en continue aanmoediging. Jij weet als geen ander dat het combineren van klinisch werk en onderzoek doorzetting en organisatie vraagt. Jij hebt me voor deze thesis gemotiveerd en mij bovendien de gelegenheid en de ondersteuning gegeven om dit werk te vervolmaken. Jouw begeestering voor het onderzoek werkt prikkelend.

I'm also grateful to my co-promotor, Dr. S. Gelsomino. Dear Sandro, I'd like to thank you for your devoted help, even far beyond the expected: your passion and enthusiasm for research have encouraged me. You watched over the momentum and kept the work pace of this thesis going. And since we have a hobby in common, Bach, you as a music collector, I as an amateur musician, I promised you to study a part of Partita II for violin: I've studied the Allemanda and Giga so far...

Orlando, I would like to thank you for your ingenious solutions when we encountered technical or statistical inconveniences and for your infinite patience in creating the graphs and figures.

Fabiana, thank you very much for the echocardiographic measurements. I acknowledge that it was a huge and punctual work.

I'm grateful to all co-authors for their contribution in the manuscripts.

Prof. J. Douglas and Dr. J. Wilson, thank you for the English revision of the chapters.

Lysette, de punctuele organisatie van mijn promotie is te danken aan jouw goede zorgen. Dank je ook voor alle informatie en jouw gedreven werk achter de schermen, je hebt me prima begeleid.

Wetenschappelijk onderzoek is een continue verrijkende wisselwerking tussen teamleden. In die zin wil ik ook het research team danken van Prof. F. Prinzen, Prof. P. De Jaegere en Drs. P. Houthuizen. Beste Frits, Peter en Patrick, jullie dank ik hartelijk omdat onze discussies over onderzoek, zij het dan rond onderwerpen die niet altijd direct aan deze thesis gelinkt zijn, zeer inspirerend werken.

Lieve familie en vrienden, bijzonder veel dank voor jullie begrip tijdens mijn grote afwezigheid. Jullie enthousiasme, gemeende interesse en onvoorwaardelijke steun hebben me gestimuleerd om mijn thesis te vervolledigen. Avondlijk filosoferen rond 
mogelijke stellingen, vergezeld van lekker eten en een goed glas wijn, waren welkome ontspanningen. Dan en Tom, dank voor jullie inspirerende discussies.

Lieve zussen, jullie wil ik speciaal danken voor jullie bereidheid en beschikbaarheid. Samen met onze ouders hebben jullie vol vertrouwen de evolutie van mijn thesis willen mee volgen. Birte, dank je voor de illustratie van een van je prachtige kunstwerken op de omslag van dit proefschrift. Karen, jouw inzicht tijdens onze gesprekken waren verhelderend. Dank je voor de coachende ondersteuning.

Lieve Mama en Papa, ik dank jullie heel speciaal voor de kansen, het vertrouwen en de liefdevolle steun. Ik dank jullie ook graag voor de logistieke ondersteuning, zowel voor de opvang van de kinderen, als voor het gebruik van jullie huis: de vertrouwelijke rust van Zeedorp was steeds een bron van inspiratie en stimulans van studie en concentratie.

Mijn grootste dank gaat uit naar mijn liefdevol gezin. Tomas, lieve schat, jouw toegenegen geduld wil ik het meest waarderen: jij hebt de successen en de tegenslagen tijdens het onderzoek van kortbij mee beleefd en hebt me steeds gewezen op het uiteindelijke doel van dit werk. Jij hield mij gefocust. Ik ben je bijzonder dankbaar voor de ruimte die je mij hebt gegeven om deze thesis af te werken. Ook aan onze lieve schatten, Carmen-Anne en Pieter-William, of anders gezegd, mijn Rattekes: ik ben jullie dankbaar voor jullie aanmoedigende steun, jullie eindeloos geduld en vooral ook de mooie momenten van ontspanning. Ik hou van jullie. 
DANKWOORD

Lieve schat,

Tongeren, Aprid La13 Licre nomá,

Ret prote trots delen we de vieupde bij het af werken wen dere thesis. dit huroromstuk. Je paf of en toe te kewnen dat ji dachter misshien vect un pelaten te hesben maen ji krijgt en eoved vor tang: het weter schappelih

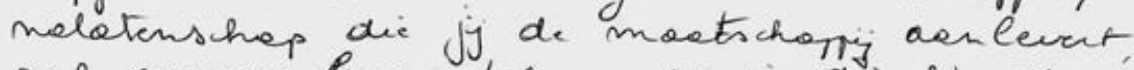
ral mensen levens helpen, en in die $\sin$ de levers hualireit van vele petienter en hun femieies perbetecen. Het is een eervolle rook on doer te kuranen op tery highen. Ret piur promotic grijpen we dan sok proeg de lian oer on pereijk ans respect to Juiten voon juw onvermoecbere imect von de patienten, thun farnilies, en bj witbraiding, de weterschop.

Jow gedrever heis is trowuen eer zook dreed in joun leven. Volgers focretes is Excelentic en kunst die men ewkel bereikt don de. consterte bexpening. we zijn wat we bij herhalimp witvoeden Evellentie is pern eer malfo acte, fet is een powonite. "Wr dwever doorom stellen dot dit weik us hender peen toevellipheid is maen een uitizo bar jour canstont streven maer verbetering: Je tont dit ook thuis in jom kookkunit, de menier urorosji de riool ken bespéter, stécas de perfect gostivoum sont en is olle especter ver hat
teven.

We rign echt Jies of je!

Rogiciat Rame propiciet Pieve Schet!

245 



\section{Curriculum vitae}

Leen Van Garsse is geboren op 1 oktober 1970 te Sint-Niklaas, België. In 1987 behaalde zij het diploma Algemeen Secundair Onderwijs in de richting Latijn-Wiskunde aan de OLV-Presentatie te Sint-Niklaas. Haar opleiding tot Kandidaat in de Geneeskunde volgde zij aan de Universiteit van Hasselt. De doctoraatsopleiding geneeskunde vervolgde zij aan de Katholieke Universiteit van Leuven, waar zij in 1995 het Artsendiploma behaalde met de grootste onderscheiding (summa cum laude). Zij vervolgde haar opleiding binnen de specialisatie Algemene Heelkunde aan de KULeuven, onder leiding van Prof.Dr. P. Broos. Tijdens deze 6 jaar durende opleiding werkte zij twee jaar in het SintNorbertus ziekenhuis te Duffel (onder leiding van Dr. J. Deleersnijder), twee jaar in het AZ Sint-Blasius te Dendermonde (onder leiding van Dr. G.Leman) en vervolgens de laatste twee jaar in KULeuven (onder leiding van Prof. Dr. P. Broos). In 2001 behaalde zij daar het diploma tot Specialist in de Heelkunde. Zij vervolgde onmiddellijk haar opleiding in de Cardiale Heelkunde (onder leiding van Prof. Dr. W. Daenen) en behaalde in 2003 het bekwaamheidsdiploma in de Cardiale Heelkunde. Van 2003 tot en mei 2004 werkte zij als staflid op de dienst Cardiale heelkunde te UZ Leuven, met een bijzondere aandacht voor klepchirurgie en congenitale hartchirurgie. Zij deed wetenschappelijk onderzoek in het labo experimentele heelkunde onder leiding van Prof. Dr. W. Flameng. Van juni 2004 tot en met augustus 2004 werkte zij te OLV Ziekenhuis te Aalst (onder leiding van Dr. H. Vanermen) om zich verder te bekwamen in de minimaal invasieve klepchirurgie. Vanaf september 2004 tot op heden is zij staflid cardiothoracale heelkunde in het Universitair Medisch Centrum te Maastricht (onder leiding van Prof. Dr. J. Maessen). In augustus 2005 verkreeg zij de Nederlandse erkenning tot cardiothoracaal chirurg.

Zij heeft zich toegelegd op minimaal invasieve thoraxingrepen: opcab, robot chirurgie en minimaal invasieve klepchirurgie. Begin 2008 startte zij samen met Dr. V. van Ommen het THI (Transcatheter Heart Valve Intervention) programma in het MUMC te Maastricht wat zij uitbouwde tot een internationaal erkend THI-centrum. Sinds 2011 is zij door de wetenschappelijke verenigingen van cardiothoracaal chirurgen (NVT) en cardiologen (NVVC) Nederland, gemandateerd stichtend voorzitter van de werkgroep THI, in co-voorzitterschap met Dr. G. Mast (interventiecardioloog te Nieuwegein). Daarnaast is zij lid van de kwaliteitscommissie van de Nederlandse vereniging voor thoraxchirurgie (NVT).

Naast haar onderzoek binnen hartfalen, in samenwerking met Dr. S. Gelsomino (Florence, Italië), is zij lid van het TAVI research team, in samenwerking met Prof. Dr. F. 
Prinzen, Prof. Dr. P. de Jaegere en Drs. P. Houthuizen (Maastricht/Rotterdam/Eindhoven).

Sinds 2009 is zij internationaal proctor voor TAVI (Transcatheter Aortic Valve Implantation) en leidde centra op in Europa, het Midden-Oosten en Latijns America. Zij behoort tot de internationale training faculty bij Edwards Lifesciences te Nyon, Zwitserland.

Leen Van Garsse is gehuwd en heeft twee kinderen. Zij volgde een ruime muzikale opleiding (piano, viool en zang) en concerteert kamermuziek als violiste in het strijkkwartet CaVA. 


\section{List of publications}

\section{Publications}

Hybrid coronary revascularization as a safe, feasible and viable alternative to conventional coronary artery bypass grafting: what is the current evidence? AJFP Verhaegh, RE Accord, L Van Garsse, JG Maessen. Minimally invasive surgery. 2013. doi:10.1155/2013/142616.

Why permanent pacemaker implantation after transcatheter aortic valve implantation does not affect longterm clinical outcome. P Houthuizen, $R$ van der Boon, L Van Garsse, F Prinzen, P De Jaegere. J Am Coll Cardiol. 2012;60(22):2339-2340.

Left ventricular strain in chronic ischemic mitral regurgitation in relation to tethering pattern. S Gelsomino, L Van Garsse, F Lucà, O Parise, Emile Cheriex, CM Rao, GF Gensini, J Maessen. J Am Soc Echocardiogr. 2013. Epub 2013/02/19.

L Left Atrial Strain and Strain Rate Before and Following Restrictive Annuloplasty for Ischemic Mitral Regurgitation Evaluated By Two-Dimensional Speckle Tracking Echocardiography. L Van Garsse, S Gelsomino, F Lucà, O Parise, Emile Cheriex, CM Rao, GF Gensini, J Maessen. Eur Heart J Cardiovasc Imaging. 2012. Epub 2012/10/12.

Left Ventricular Dyssynchrony is Associated with Recurrence of Ischemic Mitral Regurgitation After Restrictive Annuloplasty. L Van Garsse, S Gelsomino, F Lucà, O Parise, R Lorusso, E Cheriex, S Caciolli, E Vizzardi, CM Rao, R Carella, GF Gensini, J Maessen. Int J Cardiol. 2012. Epub 2012/10/10.

Minimally Invasive Mitral Valve Surgery: a systematic review. F Lucà, L Van Garsse, CM Rao, O Parise, R Carella, R Lorusso, JG Maessen, S Gelsomino. Minimally Invasive Surgery. 2013. doi:10.1155/2013/179569.

Systolic Papillary Muscle Dyssynchrony Predicts Recurrence of Mitral Regurgitation in Patients With Ischemic Cardiomyopathy (ICM) Undergoing Mitral Valve Repair. L Van Garsse, S Gelsomino, O Parise, F Luca, E Cheriex, R Lorusso, E Vizzardi, CM Rao, GF Gensini, J Maessen. Echocardiography. 2012. Epub 2012/08/18.

Tethering symmetry reflects advanced left ventricular mechanical dyssynchrony in patients with ischemic mitral regurgitation undergoing restrictive mitral valve repair. L Van Garsse, S Gelsomino, E Cheriex, F Lucà, CM Rao, O Parise, GF Gensini, J Maessen. Ann Thorac Surg. 2012;94(5):1418-1428.

Left bundle brach block induced by transcatheter aortic valve implantation increases risk of death. P Houthuizen, L Van Garsse, TT Poels, $P$ de Jaegere, RMA van der Boon, BM Swinkels, JM ten Berg, F van der Kley, MJ Schalij, J Baan Jr, R Cocchieri, 
GRG Brueren, AHM van Straten, P den Heijer, M Bentala, V van Ommen, J Kluin, PR Stella, JG Maessen, FW Prinzen. Circulation. 2012;126(6):720-728.

Blood transfusion and the risk of acute kidney injury after transcatheter aortic valve implantation. Nuis RJ, Rodes-Cabau J, Sinning JM, Van Garsse L, Kefer J, Bosmans J, Dager AE, van Mieghem N, Urena M, Nickenig G, Werner N, Maessen J, Astartci P, Perez S, Beitez LM, Dumont E, van Domburg RT, de Jaeghere PP. Circulation. Cardiovasc interv. 2012;5:680-688.

The importance of anterior leaflet tethering in predicting recurrence of ischemic mitral regurgitation after restrictive annuloplasty. F Luca, L Van Garsse, S Gelsomino, L Vasquez, E Cheriex, R Lorusso, A Lo Cascio, CM Rao, GF Gensini, J Maessen. Citation in European Heart Journal. 2011; 32, 1013.

Left Ventricular Dyssynchrony is Associated with Recurrence of Ischemic Mitral Regurgitation After Restrictive Annuloplasty. L Van Garsse, S Gelsomino, F Luca', E Cheriex, CM Rao, R Lorusso, GF Gensini, JG Maessen. Citations in Circulation. 2011; 124 (21 Suppl): A 15266)

Left Atrial Function Before and Following Restrictive Annuloplasty for Ischemic Mitral Regurgitation Evaluated by Two-Dimensional Speckle Tracking Echocardiography. L Van Garsse, S Gelsomino, E Cheriex, F Luca', CM Rao, R Lorusso, GF Gensini, JG Maessen. Citation in Circulation. 2011; 124 (21 Suppl): A 15601).

Left Bundle Branch Block after Transcatheter Aortic Valve Implantation Increases Risk for 1-year All-cause Mortality. L Van Garsse, P Houthuizen, TT Poels, P de Jaegere, RMA van der Boon, BM Swinkels, JM ten Berg, F van der Kley, MJ Schalij, J Baan Jr, R Cocchieri, GRG Brueren, AHM van Straten, P den Heijer, M Bentala, V van Ommen, J Kluin, PR Stella, JG Maessen, FW Prinzen. Citation in Circulation. 2011;124(21 Suppl): A11770.

The importance of anterior leaflet tethering in predicting the recurrence of ischemic mitral regurgitation after restrictive annuloplasty. L Van Garsse, S Gelsomino, F Luca, R Lorusso, CM Rao, P Stefano, J Maessen. J Thorac Cardiovasc Surg. 2012;143(4 Suppl):S54-S59.

Impact of anterior leaflet tethering on the recurrence of ischemic mitral regurgitation, LV reverse remodeling and reverse spherical remodeling after restrictive annuloplasty. S Gelsomino, L Van Garsse, F Luca, R Lorusso, E Cheriex, CM Rao, S Caciolli, E Vizzardi, E Crudeli, P Stefano, GF Gensini, J Maessen. J Am Soc Echocardiogr. 2011;24(12):1365-1375.

Mobile extracorporeal membrane oxygenation after traumatic freshwater submersion using bicaval dual lumen catheter. CC Geelen, EA Bouman, PM Roekaerts, P Breedveld, U Strauch, L Van Garsse, P Weerwind, D Donker. Intensive Care Med. 2011;37(12):2054-2055.

Randomized clinical trial comparing a thermosensitive polymer (LeGOO) with conventional vessel loops for temporary coronary artery occlusion during off-pump coronary artery bypass (OPCAB) surgery. G Wimmer-Greinecker, O Bouchot, JP Ver- 
hoye, LP Perrault, J Börgermann, A Diegeler, L Van Garsse, A Rastan. Ann Thorac Surg. 2011;92(6):2177-2183.

Occupational radiation dose during transcatheter aortic valve implantation. $\mathrm{L}$ Sauren, $\mathrm{L}$ Van Garsse, V van Ommen, G Kemerink. Catheter Cardiovasc Interv. 2011;78(5): 770-776.

Emergency Transthoracic Transapical Mitral Valve-in-valve Implantation. L Van Garsse, S Gelsomino, V van Ommen, F Luca, J Maessen. J Interv Cardiol. 2011;24(5):474476.

Percutaneous Transcatheter Valve-in-valve Implantation In Stenosed Tricuspid Valve Bioprosthesis. L Van Garsse, R ter Bekke, V van Ommen. Circulation. 2011;123(5): e219-e221.

Trans-catheter Aortic Valve Implantation: Initial results in Maastricht. B Alzand, L Van Garsse and J Maessen. Chapter in textbook EVC 2009.

Primary monophasic mediastinal, cardiac and pericardial synovial sarcoma: a young man in distress. de Zwaan C, Bekkers SC, Van Garsse LA, Jansen RL, Van Suylen RJ. Neth Heart J. 2007;15(6):226-8.

The Contegra conduit in the right ventricular outflow tract induces supravalvular stenosis. Meyns B, Van Garsse L, Boshoff D, Eyskens B, Mertens L, Gewillig M, Fieuws S, Verbeken E, Daenen W. J Thorac Cardiovasc Surg. 2004 Dec;128(6):834-8.

Preperitoneal laparoscopic hernioplasty. L Van Garsse, G Leman, J Himpens. Laparoscopic Hernia Repair: A New Standard ? Chapter in Book: Progress in surgery 21. 1994

\section{Abstract and poster presentations}

Changes in QRS Duration and Morphology Immediately After and At Long-Term FollowUp after Transcatheter Aortic Valve Implantation With the Self-Expanding and Balloon-Expandable Prosthesis. P Houthuizen, RMA van der Boon, LAFM Van Garsse, M Urena, TT Poels, N van Mieghem, BRG Brueren, J Rodés-Cabau, FW Prinzen, P de Jaegere. Poster presentation. AHA, 2013.

Incidence and mortality of left bundle branch block induced by surgical aortic valve replacement. TT Poels, P Houthuizen, LAFM Van Garsse, FW Prinzen, JG Maessen and AHM van Straten. Abstract presentation. Najaarsvergadering NVT, 2012. Utrecht, The Netherlands.

Blood Transfusion And The Risk Of Acute Kidney Injury Following Transcatheter Aortic Valve Implantation. RJ Nuis, J Rodes-Cabau, JM Sinning, L Van Garsse, J Kefer, J Bosmans, A Dager, N Van Mieghem, M Urena, N Werner, G Nickenig, J Maessen, P Astarci, L Benitez, E Dumont, R Van Domburg, P De Jaegere. Poster presentation. TCT, 2012. 
8-years experience with robotic thymectomy for any type of thymoma. M Keijzers, AMC Dingemans, H Blaauwgeers, RJ van Suylen, L Van Garsse, R Accord, M Hochstenbag, M de Baets, J Maessen. Poster presentation. TMIG, 2012. Japan.

Prognostic effects and cause of death after transcatheter aortic valve implantation induced left bundle branch block during long-term follow up. P Houthuizen, $L$ Van Garsse, TT Poels, P de Jaegere, RMA van der Boon, BM Swinkels, JM ten Berg, F van der Kley, MJ Schalij, J Baan Jr, R Cocchieri, GRG Brueren, AHM van Straten, P den Heijer, M Bentala, V van Ommen, J Kluin, PR Stella, JG Maessen, FW Prinzen. Poster presentation. ESC, Aug 2012. Munich, Germany..

Tethering symmetry reflects advanced left ventricular mechanical dyssynchrony in patients with ischemic mitral regurgitation undergoing restrictive mitral valve repair. L Van Garsse, S Gelsomino, E Cheriex, F Luca, CM Rao, JG Maessen. Poster presentation. Scientific Session STS, Jan 2012. F Lauderdale, Florida, USA.

Left ventricular dyssynchrony is associated with recurrence of ischemic mitral regurgitation after restrictive annuloplasty. L Van Garsse, S Gelsomino, F Luca, E Cheriex, CM Rao, R Lorusso, G Gensini, JG Maessen. Abstract presentation. NVT, Nov', 2011. Utrecht, The Netherlands.

Left ventricular dyssynchrony is associated with recurrence of ischemic mitral regurgitation after restrictive annuloplasty. L Van Garsse, S Gelsomino, F Luca, E Cheriex, CM Rao, R Lorusso, G Gensini, JG Maessen. Abstract presentation. Scientific Session AHA, Nov 201. Orlando, Florida, USA.

Left atrial function before and following restrictive annuloplasty for ischemic mitral regurgitation evaluated by two-dimensional speckle tracking echocardiography. $\mathrm{L}$ Van Garsse, S Gelsomino, E Cheriex, F Luca, CM Rao, R Lorusso, GF Gensini, JG Maessen. Poster presentation. Scientific Session AHA, Nov 2011. Orlando, Florida, USA.

Left bundle branch block induced by Transcatheter Aortic Valve Implantation increases risk for 1-year all-cause mortality. L Van Garsse, P Houthuizen, F Prinzen. Poster presentation. Scientific Session, AHA Nov 2011, Orlando, Florida, USA.

The importance of anterior leaflet tethering in predicting recurrence of ischemic mitral regurgitation after restrictive annuloplasty. F Luca, L Van Garsse, S Gelsomino, L Vasquez, E Cheriex, R Lorusso, A Lo Cascio, CM Rao, GF Gensini, J Maessen. Abstract presentation. ESC, 2011.

Anterior leaflet tethering: the importance in predicting recurrence of ischemic mitral regurgitation after restrictive annuloplasty. L Van Garsse, S Gelsomino, E Cheriex, F Luca, R Lorusso, CM Rao, GF Gensini, J Maessen. Abstract presentation. SHVD, June 2011. Barcelona, Spain.

Papillary Muscle systolic dyssynchrony (DYS-PAP) as new tool to identify advanced LV dysfunction in patients with ischemic dilated cardiomyopathy (IDCM). L Van Garsse, S Gelsomino, F Luca, E Cheriex, CM Rao, R Lorusso, GF Gensini, J Maessen. Abstract presentation. SHVD, June 2011, Barcelona, Spain. 
First successful transapical retrieval of a transcatheter valve after ventricular embolization. L Van Garsse, V van Ommen, J Maessen. E-Poster presentation. ISMICS, June 2011. Washington DC, USA.

Tethering Symmetry, Anterior Mitral Leaflet Angle and Papillary Muascle Systolic Dyssynchrony Are Powerful Tools To Identify Advanced LV Dysfunction in Ischemic Dilated Cardiomyopathy (IDCM). L Van Garsse, S Gelsomino, E Cheriex, F Luca, CM Rao, R Lorusso, GF Gensini, JG Maessen. Abstract presentation on Demand. AATS Mitral Conclave, May 2011. New York, USA.

The importance of Anterior Leaflet Tethering in Predicting Recurrence of Ischemic Mitral Regurgitation after Restrictive Annuloplasty. L Van Garsse, S Gelsomino, E Cheriex, F Luca, CM Rao, R Lorusso, GF Gensini, JG Maessen. Abstract Presentation. AATS Mitral Conclave May 2011. New York, USA.

Is early initiation of extracorporeal membrane oxygenation critical for rescue support in severe refractory cardiogenic shock. W Meijers, P Weerwind, J Nijs, J Vainer, M Lance, U Strauch, E Pragt, E Bouman, S Lochy, R Smets, G Geskes, L Van Garsse, S Kats, B Mochtar, P Roekaerts, J Maessen, D Donker. Abstract presentation. Dutch Society of cardiology, March 2011. Arnhem, The Netherlands.

Can minimized cardiopulmonary bypass systems be safer? Y Ganushack, E severdija, A Simons, L Van Garsse, P Weerwind. Abstract presentation and Best Paper Award 2011. The American Academy of Cardiovascular perfusion, Jan 2011 Reno, Nevada, USA.

Right ventricular myocardial function improvement following cardiac resynchronization therapy: a speckle tracking echocardiographic study. F Luca, S Gelsomino, R Lorusso, L Van Garsse, F van der Veen, M La Meir, R Carella, G Bille, GF Gensini, J Maessen. Abstract presentation. The Italian Federation of Cardiology, Dec 2010. Italy.

Echocardiographic evaluation of mitral valve repair in post-ischemic heart failure. $F$ Luca, F van der Veen, L Van Garsse, R Lorusso, R Carella, S Caciolli, G Bille, C Rostagno, V Pazzagli, J Maessen, GF Gensini, S Gelsomino. Abstract presentation. The Italian Federation of Cardiology, Dec 2010. Italy.

Effectiveness of an underbody forced warm-air blanket in preventing postoperative hypothermia after off-pump coronary artery bypass graft surgery. P Lozekoot, $\mathrm{L}$ Van Garsse, W van Mook, D Bergmans, P Roekaerts, J Heijmans. Abstract presentation. Scientific session NVT, Nov 2010. Utrecht, The Netherlands.

Transcatheter heartvalve procedures, a new era. L Van Garsse. Scientific presentation. Grand Rounds, Oct 2010, Maastricht, The Netherlands.

Randomised Clinical Trial Comparing a Thermosensitive polymer (LeGoo) versus conventional vessel loops for temporary coronary artery occlusion during off-pump coronary artery bypass surgery. G Wimmer-Greinecker, O Bouchot, J Verhoye, L Perrault, J Boergermann, A Diegeler, L Van Garsse, A Rastan. Abstract presentation. EACTS, Sept 2010. Geneva, Switzerland. 
Effectiveness of an underbody forced warm-air blanket in preventing postoperative hypothermia after off-pump coronary artery bypass graft surgery. J Heijmans, L Van Garsse, W Van Mook, D Bergmans, P Roekaerts. Poster presentation. EACTA, May 2010. Edinburgh, UK.

Thoracoscopic radical thymectomy for myasthenia gravis by a robotic assisted approach. M Keijzers, AM Dingemans, L Van Garsse, M de Baets, J Maessen. Abstract presentation. ESTS, 2010.

Succesful start of transapical aortic valve implantation program. L Van Garsse, V van Ommen, J Maessen. Abstract presentation. The annual meeting of the royal Belgian association of surgery, April 2009. Oostende, Belgium.

Minimal invasive aortic valve surgery. L Van Garsse. Scientific presentation. EVC-ECC, Feb 2009. Maastricht, The Netherlands.

Initial results with the Edwards SAPIEN transapical aortic valve: The AachenMaastricht experience. Dohmen G, Van Garsse L, Spillner JW, Hoffmann R, Maessen J, Autschbach R. Poster presentation. The German Society of Thoracic and Cardiovascular Surgery, Feb 2009. Stuttgart, Germany.

Transapical aortic valve implantation: the learning curve in Maastricht. Abstract presentation. Dutch Society of Cardiothoracic Surgeons, Nov 2008. Leiden, The Netherlands.

Optimizing thoracoscopic thymectomy by a Robotic assisted approach. Van Garsse L, Heymans J, De Baets M, Maessen J. Poster presentation. Dutch Society of Cardiothoracic Surgeons, April 2005. Amsterdam, The Netherlands.

The Contegra conduit in the right ventricular outflow tract induces supravalvular stenosis. Van Garsse L, Meyns B, Boshoff D, Eyskens B, Mertens L, Gewillig M, Fieuws S, Verbeken E, Daenen W. Abstract presentation. AATS, April 2004. Toronto, Canada.

Contegra-Venpro conduits in the right ventricular outflow tract: results after intermediate-term follow-up. Van Garsse L, Meyns B, Boshoff D, Eyskens B, Mertens L, Gewillig M, Fieuws S, Verbeken E, Daenen W. Poster presentation. AEPC, May 2003. The Netherlands.

Evaluation of supravalvular stenosis in RVOT-reconstruction with a bovine valved jugular vein graft. Van Garsse L, Meyns B, Boshoff D, Eyskens B, Mertens L, Gewillig M, Fieuws S, Verbeken E, Daenen W Abstract presentation. The Meeting of Belgian association of cardiac and thoracic surgeons, June 2002. Leuven, Belgium.

Endovascular exclusion of a proximal para-anastomotic pseudo aneurysm following an aorto-bifemoral bypass: a word of caution. L Van Garsse, A Nevelsteen. Abstract presentation. The 25 th world congress of the International Society of Cardiovascular Surgery, Sept 2001. Cancun, Mexico.

Carotid Endarterectomy: preoperative duplex versus angiography. L Van Garsse, J Duchateau, Ph De Vleeshouwer, J De Leersnijder. Abstract presentation. The 25 th 
world congress of the International Society of Cardiovascular Surgery, Sept 2001. Cancun, Mexico.

The accuracy of the preoperative carotid duplex. L Van Garsse, J Duchateau, Ph De Vleeshouwer, J De Leersnijder. Abstract presentation. The annual meeting of the Belgian association of vascular surgeons, 2002. Brussels, Belgium.

Respiratory insufficiency in a child: inhalation of a walnut. A case report. Presentation at the RedCross Children Hospital, 1993. Cape Town, South Africa.

\section{Invited presentations}

Hybrid revasularization. EACTS meeting, April 2013. Nieuwegein, The Netherlands. Indication for TAVI - according to the latest clinical results. Cardiovascular Radiology from NVVR, Jan 2013. Curacao, The Netherlands Antilles.

Indication for TAVI. Symposium for the inauguration Prof.Dr. De Jaeghere, Jan 2013. Rotterdam, The Netherlands.

From procedural success to sustained patient benefit: the important role of the proctor. Edwards Lifesciences proctor meeting, Nov 2012. Nyon, Switzerland.

Transapical TAVI approach: my standard of care. Training session Edwards Lifesciences, EACTS 2012. Barcelona, Spain.

Transcatheter aortic valve implantation: transapical or direct aortic approach. Lunch session Edwards Lifesciences, EACTS 2012. Barcelona, Spain.

Transapical TAVI approach. Meet the expert. Lunch session Jena Valve, EACTS 2012. Barcelona, Spain.

Evolution of team approach during TAVI procedures. EACTA, May 2012. Amsterdam, The Netherlands.

Hybrid coronary revascularization (Robotic surgery). De Novo Symposium, April 2012. Zwolle, The Netherlands.

TAVI Talk Winter edition, Edwards Lifesciences. Editorial by L Van Garsse

Invited speaker Intracardiac Echocardiography in TAVI: fast and easy - safe and simple. EuroPCR, May 2011. Paris, France

Integration TAVR into surgical therapy of Aortic Valve disease: the surgeon's perspective. GI2 Global Summit in interventions and innovations, April 2011. Sao Paulo, Brazil

Recent learnings and case examples using the Edwards SAPIEN XT THV. JIM, Febr 2011. Rome, Italy.

First successful transapical retrieval of a transcatheter aortic valve, after ventricular embolization. European proctor's meeting Edwards Lifesciences, Nov 2010. Nyon, Switzerland

Hartkleppen ingebracht via een catheter. FNT, Sept and Nov 2010. Noordwijkerhout, The Netherlands. 
Het inbrengen van een hartklep via de halsader. Nederlandse OK dagen, Oct 2010. Amsterdam, The Netherlands.

TAVI and ICE from a surgeon's perspective. ESC, Aug 2010. Stockholm, Sweden.

Transapical and transfemoral aortic valve implantation. EuroPCR, May 2010. Paris, France.

Minimally invasive mitral valve surgery. EuroPCR, May 2010. Paris, France.

Valve procedures in the hybrid room: the multidisciplinary team approach. International meeting Sorin, Nov 2009. Breda, The Netherlands.

Presentation on high risk TAVI procedures - how to be prepared. First international proctor meeting Edwards Lifesciences, Jan 2009. Paris, France.

Catheter-Based Aortic Valve Implantation. Symposium of referral centres of MUMC, Nov 2008. Maastricht, The Netherlands.

\section{Moderator - live operations}

Moderator in scientific session: Transapical TAVI and other surgical transcatheter techniques. EuroPCR, May 2013. Paris, France.

Moderator in scientific session: TAVI preferred Front Door approach. EACTS, Sept 2012. Barcelona, Spain.

Program co-director EACTA, May 2012. Amsterdam, The Netherlands.

Program co-director EVC-ECC, Feb 2010. Maastricht, The Netherlands.

Moderator in scientific session: Minimally invasive heart valve procedures. EVC-ECC, Feb 2010. Maastricht, The Netherlands.

Moderator: First international expert meeting Edwards THI, April 2009. Breda, The Netherlands.

Live operation: transapical aortic valve implantation. EVC-ECC, Feb 2009. Maastricht, The Netherlands.

Moderator scientific session: Minimally invasive heart valve procedures. EVC-ECC, Feb 2009. Maastricht, The Netherlands.

Moderator scientific session: NVT, Nov 2008. Leiden, The Netherlands. 